\title{
Recommendations from the Reducing the Inadvertent Spread of Retracted Science: Shaping a Research and Implementation Agenda Project
}

Jodi Schneider*, Nathan D. Woods, Randi Proescholdt, Yuanxi Fu, and the RISRS Team July 2021

*Corresponding author: jodi@illinois.edu and jschneider@pobox.com

\section{Table of Contents}

$\begin{array}{ll}\text { EXECUTIVE SUMMARY } & 3\end{array}$

$\begin{array}{ll}\text { Recommendations } & 4\end{array}$

INTRODUCTION

RISRS Project Goals and Assumptions $\quad 6$

Framing the Problem $\quad 8$

$\begin{array}{ll}\text { Scope of this Document } & 9\end{array}$

$\begin{array}{ll}\text { THE RISRS PROCESS } & 10\end{array}$

Scoping Literature Review 10

$\begin{array}{ll}\text { Interviews } & 11\end{array}$

Workshop, Dissemination, and Implementation 11

LITERATURE REVIEW AND CURRENT AWARENESS 11

Reasons for Retraction $\quad 11$

Formats and Types of Retraction $\quad 13$

Field Variation $\quad 15$

Continued Citation of Retracted Papers: What Went Wrong? 20

Visibility of Retraction Status $\quad 24$

Inconsistent Retraction Metadata $\quad 25$

Quality of Retraction Notices $\quad 26$

Literature Review Conclusions $\quad 28$

DEFINING PROBLEMS AND OPPORTUNITIES 29

PROBLEMS AND OPPORTUNITIES DESCRIBED BY THE EMPIRICAL LITERATURE ON RETRACTION

Problem Themes Described by the Empirical Literature on Retraction 30

Opportunity Themes Described by the Empirical Literature on Retraction 32

Problem Definition Described by the Empirical Literature on Retraction 35 
THEMES FROM STAKEHOLDER INTERVIEWS $\quad 36$

Problem Frameworks based on Stakeholder Interviews $\quad 36$

Contentious Themes based on Stakeholder Interviews 38

The Purpose of Retraction According to Stakeholder Interviews 38

Changing the Scholarly Record $\quad 39$

The Harms of Retraction According to Stakeholder Interviews 39

The Character of Reform According to Stakeholder Interviews 40

SYNTHESIZING THE PROBLEMS AND OPPORTUNITIES 41

Aligning Opportunity Pathways $\quad 41$

Defining the Scale and Scope of the Problem 42

RECOMMENDATIONS 43

1. Develop a Systematic Cross-industry Approach to Ensure the Public Availability of

Consistent, Standardized, Interoperable, and Timely Information about Retractions.

Individual Recommendations for Consistent, Standardized, Interoperable, and Timely

Information about Retractions

2. Recommend a Taxonomy of Retraction Categories/Classifications and Corresponding

Retraction Metadata that can be Adopted by All Stakeholders.

Individual Recommendations for Taxonomy and Metadata 46

3. Develop Best Practices for Coordinating the Retraction Process to Enable Timely, Fair, and Unbiased Outcomes.

Individual Recommendations for Coordinating the Retraction Process

4. Educate Stakeholders About Publication Correction Processes including Retraction and about Pre- and Post-Publication Stewardship of the Scholarly Record.

Individual Recommendations for Educating Stakeholders 48

$\begin{array}{ll}\text { Researcher Education } & 48\end{array}$

$\begin{array}{ll}\text { Authors } & 49\end{array}$

Topic Editors $\quad 50$

Editorial Education $\quad 50$

IMPLEMENTATION OF THE RECOMMENDATIONS 52

1. Implementation Actions for Recommendation \#1: Develop a systematic cross-industry approach to ensure the public availability of consistent, standardized, interoperable, and timely information about retractions.

2. Implementation Actions for Recommendation \#2: Recommend a taxonomy of retraction categories/classifications and corresponding retraction metadata that can be adopted by all stakeholders.

3. Implementation Actions for Recommendation \#3: Develop best practices for coordinating the retraction process to enable timely, fair, and unbiased outcomes.

4. Implementation Actions for Recommendation \#4: Educate stakeholders about publication correction processes including retraction and about pre- and post-publication stewardship of the scholarly record. 
1. Research Actions for Recommendation \#1: Develop a systematic cross-industry approach to ensure the public availability of consistent, standardized, interoperable, and timely information about retractions.

2. Research Actions for Recommendation \#2: Recommend a taxonomy of retraction categories/classifications and corresponding retraction metadata that can be adopted by all stakeholders.

3. Research Actions for Recommendation \#3: Develop best practices for coordinating the retraction process to enable timely, fair, and unbiased outcomes.

4. Research Actions for Recommendation \#4: Educate stakeholders about publication correction processes including retraction and about pre- and post-publication stewardship of the scholarly record.

5. Research Actions Related to RISRS Process Outcomes.

6. Research Actions Related to RISRS Citation Research.

CONCLUSIONS

Implementation Strategy

A Call to Implement and Collaborate

LIST OF APPENDICES

\section{Note: Previous versions of this document appeared in draft as documents at F1000Research, as follows:}

Draft March 14, 2021

Schneider J, Woods ND, Proescholdt R et al. Reducing the inadvertent spread of retracted science: Shaping a research and implementation agenda [version 1; not peer reviewed]. F1000Research 2021, 10:211 (document) (https://doi.org/10.7490/f1000research.1118522.1)

Draft, April 27, 2021

Schneider J, Woods ND, Proescholdt R et al. Reducing the inadvertent spread of retracted science: Shaping a research and implementation agenda [version 1; not peer reviewed]. F1000Research 2021, 10:329 (document) (https://doi.org/10.7490/f1000research.1118546.1) 


\section{EXECUTIVE SUMMARY}

Retracted research is published work that is withdrawn, removed, or otherwise invalidated from the scientific and scholarly record. This may occur for many different reasons, which can include error, misconduct, or fraud. Retracting research is intended to stop its continued citation and use, but many retracted papers continue to be used.

Retracted research that is integrated into the scientific publication network via citations-either before or after retraction-enables the inadvertent propagation of potentially unsupported or fabricated data, fundamental errors, and unreproducible results, or can lead to misattribution of results or ideas (e.g., in cases of retraction due to dual publication, plagiarism, or ownership). Research over the past decade has identified a number of factors contributing to the unintentional spread of retracted research. Many retracted papers are not marked as retracted on publisher and aggregator sites, and retracted articles may still be found in readers' PDF libraries, including in reference management systems such as Zotero, EndNote, and Mendeley. Most publishers do not systematically surveil bibliographies of submitted manuscripts, and most editors do not query whether a citation to a retracted paper is justified. When citing retracted papers, authors frequently do not indicate retraction status in bibliographies or in-text citations.

The goal of the Reducing the Inadvertent Spread of Retracted Science: Shaping a Research and Implementation Agenda (RISRS) project is to develop an actionable agenda for reducing the inadvertent spread of retracted science. This includes identifying how the gatekeepers of scientific publications can monitor and disseminate retraction status and determining what other actions are feasible and relevant.

The RISRS process included an exploratory environment scan, a scoping review of empirical literature, and successive rounds of stakeholder consultation, culminating in a three-part online workshop (October 26, November 9, and November 16, 2020) that brought together a diverse body of 70 stakeholders to engage in collaborative problem solving and dialogue. Workshop discussions were seeded by materials derived from stakeholder interviews and short original discussion pieces contributed by stakeholders. The online workshop resulted in a set of recommendations to address the complexities of retracted research throughout the scholarly communications ecosystem. Recommendations were iteratively updated and developed through a series of surveys and drafts as well as at a followup meeting online February 16, 2021.

The RISRS team solicited feedback from presentations to NISOPlus, the Society for Scholarly Publishing, and the European Association of Science Editors. Implementation actions have started through a COPE task force on taxonomy and discussions about a proposed National Information Standards Organization (NISO) Work Item. We welcome your feedback via the project website https://infoqualitylab.org/projects/risrs2020/ or by email to jodi@illinois.edu. We encourage you to disseminate these recommendations and to envision how you, in your role, and in collaborative partnerships, can make a difference. For instance, you might help form a professional working group to further develop or refine these recommendations; present about retraction and related issues at professional and academic meetings; take on an implementation or policy project; or outline further research to be conducted. 


\section{Recommendations}

1. Develop a systematic cross-industry approach to ensure the public availability of consistent, standardized, interoperable, and timely information about retractions.

2. Recommend a taxonomy of retraction categories/classifications and corresponding retraction metadata that can be adopted by all stakeholders.

3. Develop best practices for coordinating the retraction process to enable timely, fair, unbiased outcomes.

4. Educate stakeholders about publication correction processes including retraction and about pre- and post-publication stewardship of the scholarly record. 


\section{INTRODUCTION}

Retracted research is published work that is withdrawn, removed, or otherwise invalidated from the scientific and scholarly record. An article may be retracted due to the unreliability of the reported research or due to issues such as duplicate publication, plagiarism, unethical research, peer review manipulation, or use of data without permission, as well as due to honest error. Retracting research is intended to stop its continued citation ${ }^{1}$ and use, but many retracted papers continue to be used.

Retracted papers may be perpetuated into the scholarly publication network via citations both before and after retraction, which inadvertently propagates the reliance on articles that have been deemed as problematic. In many cases, this can lead to continued use or referencing of potentially faked data, fundamental errors, and unreproducible results.

Between 2003 and 2009, hundreds of thousands of people were put at risk of life-threatening side effects from taking two blood pressure medications in combination, after a fraudulent trial reported that the combination was better than either drug alone (Naik, 2011; Steen, 2011). The retracted paper is still in the top $1 \%$ of most cited articles with 932 citations in Scopus as of January 2021. Its pre-retraction citations include a 1-page journal club summary published by a US top society for primary care physicians (Weise, 2003); despite being prominent in search engine results, the journal club page has not been updated to reflect the retraction. ${ }^{2}$ Postretraction citation accounts for $18 \%$ (173) of the citations, including 59 reviews, and 30 articles in or after 2015. For instance, a 2014 Cochrane review (Li et al., 2014), which has been integrated into current UK and Australian clinical guidelines (National Heart Foundation of Australia \& National Blood Pressure and Vascular Disease Advisory Committee, 2016; National Institute for Health and Care Excellence, 2019), uses the retracted paper's published data without mentioning its 2009 retraction (The Editors of The Lancet, 2009).

A more recent example is illustrated by the significant role retracted research has played in the COVID-19 pandemic. In June 2020, two top-tier medical journals, the Lancet and the New England Journal of Medicine, made high profile retractions of COVID-19-related research published the previous month (Piller, 2021). Although both articles were retracted, they continue to have wide dissemination, with each receiving 1101 and 955 citations, respectively, in Google Scholar as of June 2021. Science Magazine analyzed a sample of 200 papers, finding that over half of the citing papers did not mention the retraction; many publishers and editors do not systematically check for retraction or post-publication corrections (Piller, 2021).

High-profile retractions are not limited to clinical medicine. Dutch social psychologist Diederik Stapel was found to have fabricated data, resulting in 58 retracted papers (Palus, 2015). While

\footnotetext{
${ }^{1}$ Citation of retracted publications, with explicit mention of the retraction, may be appropriate, for instance in meta-research studies analyzing retracted literature (where the publications are data). Research for this project has examined reasons for intentional citation to retracted publication (Hsiao \& Schneider, 2021). Appendix E presents existing citation standards for retracted publications.

2 This is even more concerning since the journal club summary was itself retracted as well (due to its dependence on the retracted paper it summarizes).
} 
the number of citations to Stapel's top 20 papers decreased after retraction, and even began to decrease prior to retraction due to media coverage, they were cited 102 times after their retraction, with the majority $(78.4 \%)$ citing Stapel's research positively-that is, not referencing the fact that it was based on fraudulent data (Fernández \& Vadillo, 2019). The Stapel retractions were part of a series of events that cast doubt on the methods and validity of experiments in the field of psychology, leading to significant changes in the ways that psychology research is conducted (Nelson et al., 2018).

Although retraction is relatively rare-about four papers are retracted out of every 10,000 published (Brainard \& You, 2018) —it has an outsized impact. The above examples demonstrate the urgency of organized efforts to limit the continued spread of retracted science and to ensure that scientific findings used to inform evidence-based decision-making are not rooted in retracted research.

Research over the past decade has identified a number of disparate factors contributing to the unintentional spread of retracted research. Many retracted papers are not marked as retracted on publisher and aggregator sites and many post-retraction PDFs are not watermarked (Bakker \& Riegelman, 2018); pre-retraction PDFs may be found in readers' PDF libraries including in reference management systems such as Zotero, EndNote, and Mendeley (Bar-llan \& Halevi, 2018; Davis, 2012). When citing retracted papers, authors are not required to identify retraction status in bibliographies or in-text citations. Most publishers do not systematically surveil bibliographies of submitted manuscripts, even though technology is emerging (Piller, 2021). Current mitigators such as Crossmark "Check for updates" buttons (Crossref, n.d.) and a Zotero retraction flagging system (Stillman, 2019) provide partial relief for individuals; eXstyles (Atypon Systems, 2013) and Edifix (When Does Edifix Warn Me about Retractions and Corrections (Errata)?, n.d.) warn publishers about retractions listed in Crossref and/or PubMed; and RedacTek (RedacTek, 2021) checks bibliographies of bibliographies (3 levels deep) for retracted papers. In 2020, new tools for manuscript submission systems became available, particularly Reference Check from the startup company scite, which checks bibliographies against data from Crossref and Retraction Watch during submission and review, so far integrated into Manuscript Manager, with integration coming in early 2021 for Aries (Editorial Manager) and Scholar One. ${ }^{3}$ A comprehensive solution requires interventions at multiple points: before reading, before writing, and before publishing (Davis, 2012).

\section{RISRS Project Goals and Assumptions}

The goal of the Reducing the Inadvertent Spread of Retracted Science: Shaping a Research and Implementation Agenda (RISRS) project is to develop an agenda for reducing the inadvertent spread of retracted science. This includes identifying how the gatekeepers of

\footnotetext{
${ }^{3}$ Josh Nicholson of scite notes that Retraction Watch data will be removed in September 2021 due to licensing restrictions; he expects Crossref and PubMed to be the predominant data sources going forward.
} 
scientific publications can monitor and disseminate the retraction status of a paper, ${ }^{4}$ and determining what other actions are feasible and relevant to support cross-sector collaboration.

Our inquiry was initially structured around the following questions:

1. What is the actual harm associated with retracted research?

2. What are the intervention points for stopping the spread of retraction? Which gatekeepers can intervene and/or disseminate retraction status?

3. What are the classes of retracted papers? (What classes of retracted papers can be considered citable, and in what context?)

4. What are the impediments to open access dissemination of retraction statuses and retraction notices?

Given the repeated attention to citation of retracted papers over a thirty-year period (Bolland et al., 2021; Davis, 2012; Decullier et al., 2013; Gabehart, 2005; Gray et al., 2019; Kochan \& Budd, 1992; Pfeifer \& Snodgrass, 1990; Van der Walt et al., 2020; N. Wright, 1991), we knew that the inadvertent spread of retracted science was a complicated and long standing issue. We started from the assumption that this was a "stuck" problem that would require collaboration across diverse stakeholders and, possibly, systemic changes to the way people work across scholarly communication.

We conceptualize scholarly communications as an ecosystem comprising the individuals, institutions, and processes through which research is produced, packaged, managed, disseminated, promoted, consumed and preserved into something deemed the scholarly record. Examples of individuals include publishers, editors, researchers, librarians, standards developers, funding program officers, and technologists. Examples of institutions include universities, government labs, funding bodies, publishing houses, libraries, technology companies, standards organizations, infrastructure providers, and vendors. Examples of processes include: submitting, peer reviewing, or accepting a manuscript; quality assurance; typesetting; creating metadata; depositing data; curating research products; selling, licensing, and acquiring books, journals, etc. These are embedded in material, social, and technical processes, which we conceptualized as points of intervention. By working with stakeholders from across the ecosystem, we endeavored to understand how people interact with retracted research, and encouraged stakeholders to reflect on how these interactions could be redesigned to interrupt the continued citation of retracted research in these chains of research communications.

\footnotetext{
${ }^{4}$ We use the term paper for any published item that is retracted. We focus on whole, published items such as journal articles, conference papers, book chapters, and monographs. Except where otherwise stated, we exclude items that do not lead to a Version of Record (NISO, 2008) (e.g., excluding preprints and data deposits, but including accepted manuscripts) as well as specific objects within a publication such as figures and images.
} 


\section{Framing the Problem}

Collaboration across major stakeholder groups is challenged by lack of common agreement about the scope of the problem, or the efficacy of strategies to address the issue. For example, retracted research is sometimes framed as an issue of individual misconduct or accountability. Likewise the continued citation of retracted research may be framed in terms of breakdowns in editing and publishing processes.

There are a set of incommensurate priorities. On the first day of the RISRS workshop this arose as a set of contrasting questions: "Are we trying to clean up the literature?" Or "... are we trying to reform science?" Or "... are we trying to reform scholarly publishing?" The importance of retraction as a means of "cleaning up the literature," stresses post-publication corrections of the scholarly record as a routine part of scientific progress. Alternately, retraction shows the urgency of the need and opportunity to reform science, with better research training, better methods, more transparency, and better ethical training to alleviate errors, before research is published.

Such differences in aim and perspective lead to some tension about what is needed, and what is possible. On the one hand, many call for substantive reform of the scientific publishing ecosystem itself and its role in scientific careers. On the other hand, others call for fine-tuning of current practices and processes in effect to optimize the retraction process, and clarify the role of retraction in stewarding the scientific record. This unresolved tension limits efforts to build the will and capacity to address the outsized impact of retracted research.

An additional challenge is that proposed solutions to the problems posed by retractions are often pitched in terms of one sector, workflow, or sphere of responsibility. To the extent that solutions are developed, they are often introduced in sector-specific ways. The RISRS team believes that increasing contact between stakeholders can help identify areas where collaboration is necessary and could be effective, in part by increasing stakeholders' familiarity with each others' work contexts.

Continued circulation of retracted research is just one of many interrelated topics relating to retracted research. Areas outside of the immediate scope of this study's recommendations include: broad reforms of science and scholarly publishing; broad reforms of researcher and/or editor education; and re-organization of relationships between editors, funders, and research institutions. These topics each call for more specialized recommendations beyond our present focus.

Many of the frameworks and themes that we observed in the stakeholder consultation interviews speak to issues that fall outside of the scope of the RISRS project, which is focused narrowly on the citation and use of retracted work. However, other issues regarding trust in science, or broad attitudes towards the best model of reform are important insofar as they identify background assumptions that shape questions of viability and implementation. 
The goal of this document is to present recommendations, an implementation agenda, and a research agenda. The recommendations can be used to align sector-specific problem-solving with cross-sectoral partnerships. The implementation agenda maps possible implementation pathways. The research agenda identifies priorities for future research.

\section{Scope of this Document}

This document focuses on the continued citation and use of retracted research. It is composed of five parts: a description of our process; results from our environment scan based on literature review and stakeholder interviews; four general recommendations; implementation actions; and a research agenda, aimed at the scholarly communication enterprise and researchers of scholarly communication.

Recommendations function at the level of policy and/or practice, and require inter-sectoral collaboration amongst multiple stakeholders communities.

Implementation Actions provide more discrete actions that help enact the recommendations and make them tangible over the long term. Some may be suitable for independent action by a single stakeholder group.

The Research Agenda outlines areas for ongoing evaluation and further research. Research related to retraction is carried out in a wider range of fields, and the empirical literature on retraction reviewed for this study is incredibly heterogeneous. Significant future work is needed to fully synthesize this research, and to share insights between different research domains. Continued research about retraction, and its citation and use, can provide factual evidence, evaluate test implementations of innovations in scholarly communication, and suggest new approaches to be tested in practice. This research agenda cannot be completed by a single group, or even a small number of researchers: different methods, expertise in different scholarly communities, and insights about different geographic areas are required. We present the research agenda for uptake by the meta-research community, with the aim of consolidating an explicit research program on the topic of retraction, retracted research, and the problem of its continued citation and use.

Our scope is primarily, but not exclusively the continued citation and use of retracted research. In particular, our environment scan of literature and stakeholder perspectives at times strays beyond citation and use alone. That is because, for many of the sources and people we consulted, citation and use are intertwined with a much broader frame of reference, including the goals, purpose, and meaning of retraction, and expectations regarding its implementation, and other aspects. Part of the work of this project has been to move from the widespread view of retraction as a social problem to a common stakeholder agenda specifying the range of problems associated with retracted research and its continued citation. 


\section{THE RISRS PROCESS}

The RISRS project has derived recommendations through a cross-sectoral consultation process. This section describes the process itself.

Stakeholders came from research institutions, government agencies, funding bodies, publishing houses, journals, libraries, technology companies, research infrastructure organizations, and vendors. By building up a body of engaged stakeholders, this project has worked to create opportunities to bridge the gaps between differing stakeholder perspectives, looking beyond the published literature to understand how the problem of retracted science and its continued citation is understood in multiple, often divergent, stakeholder contexts. These ongoing efforts at synthesis culminated in a three-part online workshop, on October 26, November 9, and November 16, 2020. Recommendations were iteratively updated and developed through a series of surveys and drafts as well as at a followup meeting online February 16, 2021.

Post-workshop activities have aimed to disseminate the RISRS draft recommendations widely throughout the scholarly communications industry, to integrate these recommendations into an implementation and research agenda, and to enlarge participation among a wider group of stakeholders (see Appendix A: RISRS Project Outcomes to Date).

We encourage you to disseminate these recommendations and to envision how you, in your role, and in collaborative partnerships, can make a difference. For instance, you might help form a professional working group to further develop or refine these recommendations; present about retraction and related issues at professional and academic meetings; take on an implementation or policy project; or outline further research to be conducted.

\section{Scoping Literature Review}

The scoping review was designed to systematically identify the literature on retraction, identify what we know about retraction from the empirical research literature, and to clarify what has been studied, and how it has been studied. We are particularly interested in understanding inconsistencies in methods, topics and assumptions across the body of literature.

To identify articles, we formally searched the literature in July 2019, April 2020, and February 2021 using a combination of systematic search in the PubMed, Scopus, and Web of Science databases. We supplemented these formal searches with citation-based searches in Scopus using a text and citation update tool we developed (Sarol et al., 2018) as well as manually identified items. We iteratively developed inclusion criteria focusing on identifying empirical research related to retraction (see Appendix C: Literature Scoping Review Methods and Intermediate Results).

In our synthesis prior to the workshop, we prioritized areas with direct and obvious relevance to the RISRS problems, such as citation and visibility of retraction status, and focused on 162 central papers (RISRS Bibliography v.1, 2021). All of our preliminary synthesis in the Literature Review and Current Awareness section below draws almost exclusively on 386 papers (RISRS 
Bibliography v.2.15, 2021). We shared this bibliography to enable its wider use, especially given the wide dispersion of this literature across multiple disciplines and diverse publication venues (see https://infoqualitylab.org/projects/risrs2020/bibliography/ ). We continue to synthesize this literature for formal publication, starting with items core to the 'spread of retracted science': we would welcome alerts to new or missing publications as well as offers to collaborate.

\section{Interviews}

Stakeholders from across the scholarly communications ecosystem were invited to participate $(\mathrm{N}=47)$ in semi-structured interviews. This process of consultation helped to further refine our scan of the problem terrain and to identify and incorporate different stakeholder perspectives. Interviewees discussed problems associated with retracted research, classes of retracted papers, their role in the publishing industry, and any opinions they may hold regarding the harms associated with retracted research. In addition, participants described the role retracted research plays in their professions and occupations, gatekeepers who might intervene in productive ways, and possible points of intervention to stop the spread of retracted research.

Interviews were subsequently analyzed to identify major themes, divergent points of view, common framings or assumptions, and points of agreement. The resulting analysis was used to design materials, such as the agenda, problem solving cases and discussion scaffolding, to structure participation in the stakeholder workshop (see Appendix D: Stakeholder Consultation Process).

\section{Workshop, Dissemination, and Implementation}

Organized as a cross-sectoral meeting with participants from across the scholarly communications ecosystem, the three-part workshop facilitated a series of frank, confidential discussions of retraction as a complex problem. In three four-hour online meetings, participants collaborated in a mix of plenary and small working groups to interactively define problems, challenge and reframe expectations, and proposed viable solutions (see Appendix: Stakeholder Consultation Process). The workshop has resulted in a series of cross-cutting recommendations (see Recommendations) for introducing changes to the way retracted research is framed, characterized, and coordinated amongst a variety of actors in the scholarly publishing ecosystem.

\section{LITERATURE REVIEW AND CURRENT AWARENESS}

\section{Reasons for Retraction}

The practice of retraction of scholarly literature emerged as a response to falsification and fabrication of research and research findings, with high profile cases in biomedical research (Steen et al., 2013). However, its use has expanded over time. Currently, according to COPE, "Retraction is a mechanism for correcting the literature and alerting readers to articles that contain such seriously flawed or erroneous content or data that their findings and conclusions cannot be relied upon. Unreliable content or data may result from honest error, naïve mistakes, 
or research misconduct" (COPE Council, 2019b). COPE provides editors with guidelines for when they should consider retracting a publication, and adds that "Retractions may be used to alert readers to cases of redundant publication, plagiarism, peer review manipulation, reuse of material or data without authorisation, copyright infringement or some other legal issue (eg, libel, privacy, illegality), unethical research, and/or a failure to disclose a major competing interest that would have unduly influenced interpretations or recommendations" (COPE Council, 2019b).

Several publishing and peer review issues may lead to retraction. In redundant publication, substantially similar work by the same author is published, particularly without cross reference (COPE Council, 2001). Faked peer review and the more general compromised peer review refer to problems in receiving unbiased independent reviews from qualified researchers; for example authors or their friends may create fake or assumed identities (often from non-institutional email accounts) and impersonate peer reviewers in order to provide glowing reviews quickly (Callaway, 2015; Chawla, 2019; COPE Council, 2012a, 2012b; Ferguson et al., 2014). Citation manipulation "is a problem when references do not contribute to the scholarly content of the article, and are included solely to increase citations" (COPE Council, 2019a); publications may be retracted due to deliberate, large-scale manipulation by so-called citation cartels (Oransky, $2012,2014)$, in which editors or authors disproportionately cite one another for a mutual increase in citation counts (Chakraborty et al., 2018; Fister et al., 2016; Jolly et al., 2020), as well as self-citation, coercive citation, etc. (Retraction Watch, n.d.-a). Systematic manipulation and falsification of bibliography items themselves can also lead to retraction (COPE Council, 2010). However, citation manipulation can take time to discover, and its detection is an ongoing area of research.

Another set of reasons for retraction relate to legal, ownership, and approval issues. Legal issues may include copyright infringement, breach of confidentiality, and defamation (McLean, 2013). Ownership issues such as data ownership (COPE Council, 1999) (COPE Council, 2013) and authorship disputes (COPE Council, 2018) may be intertwined. Lack of ethical approval (Retraction Watch, n.d.-c), may also lead to retraction.

Mistakes in the editorial, publishing, and production processes can also lead to retraction. Examples include mistaken publication of the same article twice (Abritis, 2016; Koziol, 2016), accidental publication of rejected manuscripts (Chawla, 2016; V. Stern, 2017), and unintentional publication of conference proceedings (McCook, 2017).

Honest error can lead researchers to retract their own work (Gewin, 2014; Retraction Watch, n.d.-b). However, there are perceived disincentives for self-retraction (Fanelli, 2016). In particular, reputational harm is also a significant concern: "The thought of having to retract an article can instill fear into the heart of scientists, who see it as equivalent to being named and shamed" (Bishop, 2018). Scientometrics research suggests that honest and dishonest retractions lead to different career impacts, as measured by the citation of other non-retracted papers the authors had previously published (Azoulay et al., 2017; Lu et al., 2013). Those who self-retract concur: According to an interview study with 14 authors, "having to retract their own 
publication made them concerned for their scientific reputation and career, often causing considerable stress and anxiety," (Hosseini et al., 2018). Yet "contrary to the researchers' expectations" their reputations were, if anything, improved following the self-retraction (Hosseini et al., 2018). These dynamics are reflected in experience reports collected for the RISRS project (Ronald, 2013; Strand, 2020) as well as in conference reports (Enserink, 2017; Mann, 2017) and published experience reports (Kullgren \& Carter, 2015). Self-retraction is currently seen as "a heroic act" (Vuong, 2020); culture change ${ }^{5}$ and institutional support are needed to ensure that when "scientists detect and address flaws in work" it is seen as success, not failure (Alberts et al., 2015).

Finally, scientific issues the author does not agree with can also lead to retraction. This can include major flaws with the study design or data analysis methods that were not addressed during peer review. "Distinguishing between misconduct and scientific disagreement can be difficult because scientific disagreement involves a dispute about the norms within one's discipline or their application to a particular study." (Resnik, Jr. \& Stewart Jr., 2012). Issues of reproducibility, rigor, choices of materials, methods, and interpretations may fall into this category.

\section{Formats and Types of Retraction}

Over the years, multiple types of retraction have been made. The first of these is full retraction, in which a paper is retracted in its entirety. Retracted articles are generally expected to remain available in public view, with specific exceptions (Wiley, n.d.)

Second, a publication may be retracted and republished ${ }^{6}$, meaning that a new version, with changes incorporated, receives a different digital object identifier (DOI) (Cagney et al., 2016). The original article remains available but clearly marked as retracted, and the two versions are interlinked. Science Magazine notes that "JAMA Psychiatry in 2015 introduced the concept of retracting and replacing (Heckers et al., 2015) for a paper about a clinical trial that had pervasive errors but, once corrected, was still worth publishing. Editors at The Lancet and The

\footnotetext{
${ }^{5}$ Such culture change seems to be starting in some fields: the "loss-of-confidence" project focuses on self-correction efforts (including but not limited to retraction) from empirical reports of novel findings in psychology; they say "even the 13 submissions we ultimately received could be considered a clear success and a testament to the current introspection and self-critical climate in psychology."(Rohrer et al., 2021).

${ }^{6}$ This is referred to as "Retractedandrepublished" in the PubMed XML help (U.S. National Library of Medicine, 2021). PubMed can be searched for "corrected and republished article"[Publication Type].

COPE writes: "In some instances, journals may wish to work with authors to concurrently retract an article that was found to be fundamentally flawed while simultaneously publishing a linked and corrected version of the work. This strategy of 'retract and republish' is not commonly used, but may provide an opportunity for journals and authors to transparently correct the literature when a simple correction cannot sufficiently address the flaws of the original article (eg, see Retraction and republication - a new tool for correcting the scientific record? European Science Editing (http://b.link/ese)). In this instance, the original article should not be completely removed or 'replaced', but should be retained and linked to" (COPE Council, 2019b).
} 
Lancet Respiratory Medicine have instituted retraction and republication (The Editors of The Lancet, 2015)" (Enserink, 2017).

A related, but problematic form of retraction is when a new version, with changes incorporated, receives the same $\mathrm{DOI}$ as a retracted, previous version. In this case, the original retracted article does not remain available on the publisher website and cannot be distinguished by DOI from the new one. The terminology does not seem to be consistent (Marasović et al., 2018).

Partial retraction (not including corrections and errata) is no longer considered good practice. The US National Library of Medicine says "Corrections, corrigenda, addenda, and partial retractions (such as for a single graph, statement, table or image) for previously published articles are all uniformly considered by NLM to be errata" (U.S. National Library of Medicine, 2018).

Finally, withdrawal is closely related to retraction, and may be alternately distinguished from it or considered a subtype of retraction. Withdrawal may include removing the article entirely, exceptionally. Currently practice around articles in press is inconsistent; for instance, Elsevier takes down articles in press not only for administrative reasons but also for failures in publication ethics ${ }^{7}$, and Wiley suggests that early versions of articles with DOls but without full bibliographic may not yet constitute the Version of Record ${ }^{8}$ Yet COPE guidelines ${ }^{9}$ suggest that articles in press be handled in the same way as other articles being retracted. Lag time between online publication and formal publication in an issue varies, but is generally measured in months (Tort et al., 2012) and sometimes more than a year (Al \& Soydal, 2017) ${ }^{10}$. Articles in press, like preprints, may be read and cited before they are assigned an issue (Haustein et al., 2015).

\footnotetext{
${ }^{7}$ Elsevier: "Withdrawn means that the article content (HTML and PDF) is removed and replaced with a HTML page and PDF simply stating that the article has been withdrawn according to the Elsevier Policy on Article in Press Withdrawal with a link to the current policy document" (Elsevier, n.d.). Retraction Watch raises concerns about the lack of transparency of this approach (Oransky, 2013, 2018b).

8 "Where an Accepted Article (which represents an early version of an article) is to be retracted, because for example it contains errors, has been accidentally submitted twice or infringes a professional ethical code of some type, it may be deleted. This is because, whilst an Accepted Article will have been allocated a Digital Object Identifier (DOI), it does not constitute the Version of Record as it will not yet have been formally published and does not yet carry complete bibliographic information" (Wiley, n.d.).

9 "Posting an "in press" or final version of an article online usually constitutes publication even if the article has not appeared (or will not appear) in print. If an article is retracted before it appears in the print or online version of a journal, or if the journal does not publish in print, the online version of the article should be retained with a clear Notice of Retraction and it should be included in bibliographic databases (eg, with a digital object identifier (DOI) or other permanent citation). Retaining the original work ensures transparency of the published record, as online versions may have been accessed and cited by researchers prior to retraction" (COPE Council, 2019b).

${ }^{10}$ For instance, the oldest five Articles in Press for Gaceta Sanitaria were available online between September and December 2019, as of April 24, 2021 https://www.sciencedirect.com/journal/gacetasanitaria/articles-in-press?page $=6$
} 


\section{Field Variation}

Retraction and its implications have been studied within several different fields or disciplines. Field-specific papers provide information about the prevalence of retraction, the rate of postretraction citation, the obstacles to preventing the spread of retracted science, retraction policies, and many other pieces of information that may differ from discipline to discipline. 95 of our 386 included publications study retraction within specific subject areas, offering a variety of data and perspectives. However, there are also gaps where future research may be useful.

Table 1. Disciplines studied in retraction research

\begin{tabular}{|l|c|}
\hline Field & $\begin{array}{c}\text { Number } \\
\text { of articles }\end{array}$ \\
\hline Medicine/Biomedicine/Health and Life Sciences & 73 \\
\hline Business/Finance/Economics/Management & 7 \\
\hline Chemistry and Materials Science & 4 \\
\hline Engineering & 3 \\
\hline Humanities & 3 \\
\hline Library and Information Science & 2 \\
\hline Computer Science & 1 \\
\hline Tourism and Hospitality & 1 \\
\hline Mathematics & 1 \\
\hline Grand Total & 95 \\
\hline
\end{tabular}

Table 1 shows the fields analyzed by the literature we identified (Proescholdt, 2021). Despite several exclusions (discussed below), the category of Medicine/Biomedicine/Health and Life Sciences is by far the most researched in the study of retraction. Few articles-22-focus on fields outside of medicine and biomedicine, covering Business/Finance/Economics/Management, Chemistry and Materials Science, Engineering, Library and Information Science (including one focused on medical libraries), the Humanities, Mathematics, Tourism and Hospitality, and Computer Science.

Table 1 excludes studies that used field-specific data sources-MEDLINE/PubMed (49), ORI misconduct cases (11), Cochrane Reviews (3), KoreaMed (2), Embase (1), FDA inspections (1), and IndMed (1) —without narrowing their search with field-related keywords or controlled vocabulary. Some of these studies focused more on the database than the field, and the 
contents of each data source vary, leading to a different understanding of 'the field' covered. Some researchers may have selected MEDLINE/PubMed because they sought to study the biomedical field, whereas others may have used it because it is freely available, large in scope, and easy to use.

In Table 1, we also did not consider studies on retracted COVID-19 publications to be fieldspecific, as retraction in COVID-19 has been explicitly described as covering multiple fields of study; one of the articles we analyzed found that, while the majority (29/35) of retracted COVID19 articles fell within Health Sciences, others were categorized as Basic Life Sciences (3/35) and Business and Technology (3/35) (Cortegiani et al., 2021). However, it is worth noting that 14 articles included in our review focused on retractions related to COVID-19. Some commentaries note that a rush to publish articles on COVID-19 may have resulted in a greater number of problems in the literature (Bramstedt, 2020; Kodvanj et al., 2020; Matas et al., 2020; Yeo-Teh \& Tang, 2021), though others are skeptical of such conclusions and suggest it will take years before we can accurately compare the retraction rates in COVID-19 research and other areas of study (Abritis et al., 2020).

Multi-disciplinary comparisons, discussed below, have also found Medicine/Biomedicine/Health and Life Sciences to be a field with a relatively high proportion of retracted articles. Some possible reasons for the prevalence of research on retraction in the biomedical field are highprofile cases of retraction in this area, such as Wakefield, Reuben and the more recent case of Harvard researcher Piero Anversa (Oransky \& Marcus, 2018), as well as the obvious consequences that can result from medical treatments influenced by fraudulent or incorrect data. These consequences are demonstrated by the case of German anaesthetist Joachim Boldt, who authored about 90 retracted articles that included fabricated results that downplayed the dangers of a substance that is "probably killing between 200 and 300 people every year in the UK" (Wise, 2013). Steen (2011) explored the consequences of the use of retracted studies in medicine, finding that 180 retracted studies involved the treatment of 9189 patients while 70,501 additional patients received treatment in secondary studies that cited those retracted studies. Steen noted that fraud can be harmful both in its impact on treatments and in public trust. In a related study, Steen (2012) found that retracted clinical trials directly involved more patients and influenced more secondary studies than other types of studies, suggesting that clinical research has a high potential for harm when data is fraudulent.

For 13 of the 73 articles in Table 1, researchers studied retraction in Medicine/Biomedicine/Health and Life Sciences broadly, by performing their data collection with some type of field limitation or screening to narrow the contents of one or more databases. The other 60 Medicine/Biomedicine/Health and Life Sciences studies narrowed their research to specific subfields as shown in Table 2. Psychology/Psychiatry, Surgery, Cancer/Oncology, and Dentistry are the most researched, with a variety of other fields also being represented. 
Table 2. Subfields represented in retraction research within Medicine/Biomedicine/Health and Life Sciences

\begin{tabular}{|l|c|}
\hline Field & Number of articles \\
\hline Psychology/Psychiatry & 7 \\
\hline Surgery & 7 \\
\hline Cancer/Oncology & 6 \\
\hline Dentistry & 6 \\
\hline Molecular Biology, Biochemistry, and Genetics & 4 \\
\hline Anesthesiology & 4 \\
\hline Nursing Science & 3 \\
\hline Pharmaceutical & 3 \\
\hline Rheumatology & 3 \\
\hline Radiology & 2 \\
\hline Rehabilitation and Sport Sciences & 1 \\
\hline Orthopaedics & 1 \\
\hline Obstetrics and Gynaecology & 2 \\
\hline General and Internal Medicine & 1 \\
\hline Health Policy and Services & 1 \\
\hline Urology & 1 \\
\hline Infection Prevention and Control & 1 \\
\hline Endocrinology and Metabolism & 1 \\
\hline Cardiology & 1 \\
\hline Emergency Medicine & 1 \\
\hline Ophthalmology & 1 \\
\hline Neurology & 1 \\
\hline Grand Total & 1 \\
\hline
\end{tabular}


We also found 55 multidisciplinary comparisons: studies that compared retraction in different fields. Many of these found that retraction is most common in medical and biological fields. For example, He (2013) analyzed science subfields in the Science Citation Index Expanded (SCIE) database of Web of Science, finding that the highest number of retractions were in the multidisciplinary sciences category, though the vast majority of the retractions in that category came from three high-impact factor journals (Nature, Science, and Proceedings of the National Academy of Sciences of the United States of America); He notes that factors such as the higher level of scrutiny on publications in these journals could affect retraction rates. The categories with the next most retractions were biosciences, biomedical research, and clinical and experimental medicine (He, 2013).

Similarly, Lu et al. (2013) found that the most retractions occurred in the "hard sciences", particularly in biomedical and multidisciplinary journals. They found lower retraction rates in the social sciences and arts and humanities, believing this to be either because of lower occurrences of error in these fields, or because of lower occurrences of the detection of error.

A study by Halevi (2020) suggests that "significant overlap with previous published research" and plagiarism are the most common reasons for retraction in the Arts and Humanities. The study cites the "essay-like" nature of works in this field as a possible reason for the prevalence of plagiarism, comparing the ease of copying and pasting content into an essay with the ease of manipulating data in the sciences.

Shuai et al. (2017), also studying data from Web of Science, found the highest rates of retraction in medical or biological fields. In an analysis of more specific topics, they found that the most retracted papers discussed gene expression and regulation of gene expression.

Other studies note medicine and biology as fields with high retraction rates, but also draw attention to other fields to watch. Interestingly, while Vuong et al. (2020) found a large portion of retractions coming from physical sciences, basic life science, and the health sciences, they also list business and technology as a field with a significant number of retractions. However, we posit that the high number of business and technology retractions might be due to the high number of retractions by the Institute of Electrical and Electronics Engineers, which the study found to be the publisher with the most retractions.

In a study drawing from 42 different databases, Grieneisen and Zhang (2012) found almost 4,500 papers retracted 1928-2011. They found the highest rates of retraction in the field of Anesthesiology, though they include the caveat that repeat offenders significantly skew this result: two researchers, Boldt and Reuben, account for $85 \%$ of the retractions from Anesthesiology journals in their study. They also suggest that retraction rates in Medicine, Chemistry, Life Sciences and Multidisciplinary Sciences are higher than those in Engineering \& Technology, Social Sciences, Mathematics, Physics, Agriculture, Earth \& Space Sciences, Ecology \& Natural Resources and Humanities. In another article, Zhang and Grieneisen (2013) note that rates of retraction due to misconduct in chemistry and non-medical life sciences were comparable to those in medical sciences. 
However, it is important to note that greater rates of retraction do not necessarily indicate greater rates of error, falsification, or fraud in a particular field. A greater number of retractions can instead indicate greater attention to scientific integrity and validity, as the scholarly community devotes greater attention to removing errors and fraud from the literature (Brainard \& You, 2018; Fanelli, 2013). The amount of attention to scientific integrity may not be the same in each field. Applied fields may gain scrutiny from a wider audience than more abstract ones, and the extent to which work is empirically grounded in evidence that is directly reported can vary between fields. The integrity issues that are easiest to surface are more likely to result in retraction. For instance, the presentation of image data in the sciences (in article figures) provides a certain level of transparency/visibility, and also a point of research reporting where errors or alterations can be introduced. Issues pertaining to image data reporting are highly represented in retractions (Bik et al., 2016), and have received significant attention (Abbott, 2019; Bik et al., 2016, 2018; Bucci, 2018; Fanelli et al., 2019), as have straightforwardly plagiarized text (Dougherty, 2020; Foltýnek et al., 2019) and statistically impossible or improbable data (Carlisle, 2012, 2017; Horton et al., 2020; Pandit, 2012). Factors such as data availability (Dafoe, 2014) and publication file formats (Labbé et al., 2020) also impact the ease of detection. By contrast, another well-known problem, misidentified cell lines, is described as widespread, but rarely results in retractions (Casadevall et al., 2014; Horbach \& Halffman, 2017; Vaughan et al., 2017). Likewise, one study on research misconduct identified during US Food and Drug Administration inspections of clinical trials found that "No corrections, retractions, expressions of concern, or other comments acknowledging the key issues identified by the inspection were subsequently published" (Seife, 2015), suggesting this is a field that may require more attention.

Variance in policies and norms within each field also factors into which fields produce the most retractions. For instance, in mathematics, incorrect proofs of challenging problems such as the Four Color Theorem and Fermat's Last Theorem continue to be publicly discussed, despite their known incorrectness. Differences in the genre of publication (articles versus conference papers versus book chapters and books) may also make a difference. Whereas retraction gained publicity in the medical sciences in the early 1980s (Kochan \& Budd, 1992), many fields have started to retract items only in the last decade or two (e.g., since 2000 or later). Retraction rates can also vary for different parts of the same field (Zhang \& Grieneisen, 2013 Tables 4 and 5).

Explaining field variation in retraction requires significant interpretation of existing studies, which generally either analyze the items that have been retracted or the authors with the most retractions. These studies do not always represent a complete picture, as many problems in the literature are likely to be undetected, in some fields more than others. The data on retraction can be skewed due to large numbers of retractions of works by individual authors or publishers in certain fields, differences in the availability of information on different fields, different publication rates, and different levels of attention and scrutiny on publications in different fields. Current research suggests that more retractions occur in the sciences, especially medicine and biology, than in other areas such as social sciences, arts, and humanities. However, existing research on retraction also focuses more attention on biomedical and life sciences. More research into non-biomedical fields, specific biomedical subfields, and the reasons for field 
variation may prove useful to understanding the phenomenon of retraction and how it is handled in different disciplines.

\section{Continued Citation of Retracted Papers: What Went Wrong?}

Retracted papers may receive a considerable number of citations. A comprehensive survey of the 1,775 retracted articles in Web of Science as of March 30, 2012 found that retracted articles on average receive 22.29 citations (total, including both pre- and post-retraction) (Chen et al., 2013). Narrowing down to the biomedical field, one study found that a set of 4,871 retracted articles published from 1959 through 2018 were cited on average a total of 35 times each in Scopus (Dinh et al., 2019). A similar number of average total citation counts, 35.1, in Web of Science, was found in oncology, a subfield of biomedicine, for a set of 1572 oncology articles in PubMed published from 2000 to 2018 (Pantziarka \& Meheus, 2019).

Papers continue to be cited after their retraction. A concerning observation is that retraction had no impact on the total number of citations: Bolboacă et al. (2019) compared Weighted Citation Indices (the number of citations divided by the citation time window) before and after retraction of retracted radiology papers taken from PubMed and found no significant difference. Neale et al. (2010) compared citations of retracted papers with adjacent unretracted ones and found no evidence that retracted papers received fewer citations. Moreover, post-retraction citations should document the retracted paper's retraction status. A study of citations of Wakefield's autism/MMR vaccine paper (partially retracted in 2004 and fully retracted in 2010) found a steady increase of the papers documenting the retraction status from 2005 (38.2\%) to 2018 (88.5\%) (Suelzer et al., 2019).

Bibliometric researchers have long suggested that not all citations should count equally; for instance Eugene Garfield mentioned "Disclaiming work or ideas of others (negative claims)" as one purpose for citing (Garfield, 1965). The term 'citation sentiment' has been taken up, particularly in the natural language processing community, following sentiment analysis, dating at least to Athar (2011). In work on post-retraction citation, the definition from Suelzer et al. (2019), is typical: negative citation "disputes, corrects or questions, or negatively evaluates cited work". Using that definition, the majority of the citations $(72.7 \%)$ to the Wakefield paper are negative (Suelzer et al., 2019). Unfortunately, this does not apply to retracted papers in general.

A series of studies have shown the disturbing fact that negative citations and citations documenting the retraction status only account for a small fraction of post-retraction citations (Table 3). It is both important and interesting to investigate why post-retraction citations of invalidated literature are predominantly positive (i.e., citing retracted work as if it were still valid). It is natural to think that low visibility of retraction status is the cause. However, studies that establish such causal relationships are still needed. By studying one retracted paper that has been repeatedly cited, Schneider et al. (2020) found that errors in database metadata and library link resolvers may have prevented users from seeing the retraction notice when they searched for this retracted paper. Balhara \& Mishra (2014) assessed compliance of retracted publications with COPE guidelines, comparing data from before the introduction of the COPE guidelines in 2009, and after. They found that the difference between pre-2010 and post-2010 retraction 
notices was small, and also found that the lack of a freely available retraction notice led to a "statistically significant" increase in post-retraction citations.

Table 3. Fraction of negative post-retraction citations and citations documenting retraction status reported by research articles

\begin{tabular}{|c|c|c|c|c|c|}
\hline Reference & $\begin{array}{l}\text { Fraction } \\
\text { of } \\
\text { negative } \\
\text { citations }\end{array}$ & $\begin{array}{l}\text { Fraction of } \\
\text { citations that } \\
\text { documents } \\
\text { the retraction }\end{array}$ & $\begin{array}{l}\text { Total number } \\
\text { of post- } \\
\text { retraction } \\
\text { citations } \\
\text { studied }\end{array}$ & $\begin{array}{l}\text { Retracted paper(s) } \\
\text { taken from }\end{array}$ & $\begin{array}{l}\text { Citation data } \\
\text { from }\end{array}$ \\
\hline $\begin{array}{l}\text { (Pfeifer \& } \\
\text { Snodgrass, } \\
1990)\end{array}$ & - & $2.9 \%{ }^{11}$ & 175 & $\begin{array}{l}82 \text { retracted papers } \\
\text { in Index Medicus }\end{array}$ & $\begin{array}{l}\text { Science Citation } \\
\text { Index }\end{array}$ \\
\hline $\begin{array}{l}\text { (Kochan \& Budd, } \\
\text { 1992) }\end{array}$ & $8.4 \%$ & $5.7 \%$ & 298 & $\begin{array}{l}\text { John Darsee's } \\
\text { publications }\end{array}$ & $\begin{array}{l}\text { Science Citation } \\
\text { Index }\end{array}$ \\
\hline $\begin{array}{l}\text { (Budd et al., } \\
\text { 1999) }\end{array}$ & -- & $\begin{array}{l}6.4 \% \\
\text { (Abridged } \\
\text { Index Medicus } \\
\text { (AIM) } \\
\text { citations); } \\
7.7 \% \text { (non- } \\
\text { AIM citation) }\end{array}$ & $\begin{array}{l}\text { AIM citations: } \\
299 ; \text { non-AIM } \\
\text { citations: } 1594\end{array}$ & MEDLINE & $\begin{array}{l}\text { Science Citation } \\
\text { Index }\end{array}$ \\
\hline $\begin{array}{l}\text { (Redman et al., } \\
2008 \text { ) one paper } \\
\text { with the highest late } \\
\text { post-retraction } \\
\text { citations; nine papers } \\
\text { with high late post- } \\
\text { retraction citations }^{12}\end{array}$ & - & $29 \% ;<3 \%$ & $96 ; 129$ & PubMed & Web of Science \\
\hline $\begin{array}{l}\text { (Neale et al., } \\
\text { 2010) }\end{array}$ & $.3 \%^{13}$ & $2.8 \%{ }^{14}$ & 603 & $\begin{array}{l}102 \text { papers named } \\
\text { in official findings of } \\
\text { scientific } \\
\text { misconduct during } \\
\text { the period of } 1993 \\
\text { and } 2001\end{array}$ & Web of Science \\
\hline
\end{tabular}

${ }^{11}$ Based on the percentage of articles studied that cited both the retracted publication and its retraction notice.

${ }^{12}$ Late post-retraction citations are those occurring at least 4 years after citation; "There were 10 papers with high late post-retraction citation rates and these were cited a total of 225 times 4 or more years after retraction (10-96). In nine of these cases, the rate of acknowledgement of the retraction in these late citations was less than $3 \%$. The paper with 96 late post-retraction citations dealt with a supposedly settled controversy with significant environmental implications and was immediately questioned by other scientists; the retraction of this paper was acknowledged in $29 \%$ of the post-retraction citations." (Redman et al., 2008)

${ }^{13}$ We chose to compare with what Neale et al. call "direct contrast," which "denotes a direct challenge of the findings reported in the affected article."

${ }^{14}$ We chose to compare with what Neale et al. call "awareness," which denotes "awareness that information from the affected article was potentially invalid and/or in need of a correction or retraction." 


\begin{tabular}{|c|c|c|c|c|c|}
\hline $\begin{array}{l}\text { (Budd et al., } \\
\text { 2011) }\end{array}$ & -- & $6 \%$ & 391 & PubMed & Scopus \\
\hline $\begin{array}{l}\text { (Bornemann- } \\
\text { Cimenti et al., } \\
\text { 2016) }\end{array}$ & -- & $25.8 \%$ & 267 & $\begin{array}{l}19 \text { papers from the } \\
\text { Scott Reuben case }\end{array}$ & $\begin{array}{l}\text { Web of Science } \\
\text { Core Collection }\end{array}$ \\
\hline $\begin{array}{l}\text { (Budd et al., } \\
\text { 2016) }\end{array}$ & -- & $4.15 \%$ & 4,917 & MEDLINE OVID & Scopus \\
\hline $\begin{array}{l}\text { (van der Vet \& } \\
\text { Nijveen, 2016) }\end{array}$ & & $3.5 \%$ & 57 & $\begin{array}{l}\text { A paper published } \\
\text { in Nature and } \\
\text { retracted in Feb } \\
2014\end{array}$ & Scopus \\
\hline $\begin{array}{l}\text { (Bar-llan \& } \\
\text { Halevi, 2017) }\end{array}$ & $5 \%$ & -- & 238 & ScienceDirect & Scopus \\
\hline $\begin{array}{l}\text { (Bolboacă et al., } \\
\text { 2019) }\end{array}$ & $2.33 \%$ & $1.07 \%$ & 559 & $\begin{array}{l}43 \text { radiology } \\
\text { diagnosis papers } \\
\text { from PubMed }\end{array}$ & Scopus \\
\hline (Hamilton, 2019) & -- & $8 \%{ }^{15}$ & 358 & $\begin{array}{l}46 \text { radiation } \\
\text { oncology papers }\end{array}$ & $\begin{array}{l}\text { Web of Science, } \\
\text { Scopus, and } \\
\text { Google Scholar }\end{array}$ \\
\hline $\begin{array}{l}\text { (Suelzer et al., } \\
\text { 2019) }\end{array}$ & $72.7 \%$ & $56 \%$ & 881 & Wakefield paper & $\begin{array}{l}\text { Web of Science } \\
\text { Core Collection }\end{array}$ \\
\hline $\begin{array}{l}\text { (Schneider et al., } \\
\text { 2020) which } \\
\text { updates (Fulton et } \\
\text { al., 2015) }\end{array}$ & $3.5 \%$ & $4.5 \%$ & 112 & $\begin{array}{l}\text { A single retracted } \\
\text { clinical trial from the } \\
\text { Matsuyama case }\end{array}$ & $\begin{array}{l}\text { Web of Science, } \\
\text { Scopus, and } \\
\text { Google Scholar }\end{array}$ \\
\hline $\begin{array}{l}\text { (Van der Walt et } \\
\text { al., 2020) }\end{array}$ & -- & $21.05 \%$ & 19 & $\begin{array}{l}33 \text { retracted } \\
\text { COVID-19 papers }\end{array}$ & iCite \\
\hline (Piller, 2021) & -- & $47.5 \%$ & 200 & $\begin{array}{l}\text { Two retracted } \\
\text { COVID-19 papers } \\
\text { related to } \\
\text { Surgisphere data }\end{array}$ & -- \\
\hline $\begin{array}{l}\text { (Hsiao \& } \\
\text { Schneider, 2021) }\end{array}$ & -- & $5.4 \%$ & 13,252 & $\begin{array}{l}\text { PubMed Central } \\
\text { Open Access } \\
\text { subset (XML only) }\end{array}$ & iCite \\
\hline $\begin{array}{l}\text { (Santos- } \\
\text { d'Amorim et al., } \\
\text { 2021) }\end{array}$ & $38 \%$ & -- & 162 & $\begin{array}{l}\text { One retracted } \\
\text { paper related to } \\
\text { Surgisphere data }\end{array}$ & Google Scholar \\
\hline
\end{tabular}

Studies of post-retraction citations also challenge our assumptions about researchers' information behavior, including their information management and general information practices. For

\footnotetext{
${ }^{15}$ Inferred from the data reported (subtraction).
} 
example, the role of mass media should not be underestimated. Reduction in post-retraction citation has been found in a few high-profile retraction cases but not those less well-reported (Mott et al., 2019). Another study using the Altmetric Attention Score (from altmetric.com), a measure based on news and social media attention and online search data, made the unique discovery that retracted papers with high Altmetric score (i.e., more online attention) were more likely to result from misconduct and also received fewer citations (Shema et al., 2019). Moreover, researchers outside a field tend to be more prone to citing retracted papers than researchers inside the field (Bornemann-Cimenti et al., 2016). Thus, there should be further studies to understand the difference between in-field and out-of-field researchers regarding how they receive information about retractions. Problematic citation behavior is another concern. If authors copy references from others without checking the original paper or retrieve papers from unofficial channels (e.g., pirate copy sites, self-archives, academic social networks) (Dubin, 2004; Simkin \& Roychowdhury, 2005; Wetterer, 2006), merely improving the visibility of retraction status in publishers' websites or databases will still be inadequate. Education of authors is important. Moreover, a reference-checking step in the publication process should be considered to be another, potentially final, defense against the unknowing citation of retracted work.

Papers citing retracted articles are not subject to any additional scrutiny, and such citations raise the concern that errors may propagate for generations into the scientific literature and damage its integrity (Schneider et al., 2020; van der Vet \& Nijveen, 2016). In fact, retracted articles in nursing science journals were found to be cited by systematic reviews as evidence, and some of the systematic reviews were published after the retraction (Gray et al., 2018). To prevent such diffusion, 2019 COPE guidelines mention that systematic reviews must consider correction or retraction following retraction of a publication they synthesize (COPE Council, 2019b). Network analysis has been applied to visualize such propagation (Chen et al., 2013; Schneider et al., 2020; van der Vet \& Nijveen, 2016). Thus, besides preventing scientists from using and citing retracted articles unknowingly, we must also ask how to deal with positive citations already existing in the archive. To develop a formal approach, Fu and Schneider (2020) introduced a new framework, the "keystone framework." It combines argumentation theory, argument-based modeling of scientific paper, and citation content analysis. Using the keystone framework, users can differentiate citations that do not impact the validity of the citing paper from citations that do impact the validity of the citing paper. In the former case, a mark can be placed next to the citation so that readers will be informed of the potential validity issues with the citation contexts. However, in the latter case, the validity of the entire citing paper is called into question. Additional measures (e.g., alerting authors to double-check their results) need to be taken to prevent science being built on "shaky" or "absent shoulders" (Azoulay et al., 2015).

However, researchers do find legitimate reasons to cite retracted papers. Retracted papers may be cited as a part of history in a review (Osman et al., 2017 ref 39), as the reason for exclusion in systematic reviews and meta-analyses (e.g., Palacio et al., 2014 ref 45 ), to justify more research effort (e.g., Sehnal et al., 2015 ref 1-4)), or to reduce the strength of evidence supporting opposing views (e.g., Thomas Manapurathe et al., 2017 refs 45 and 46). ${ }^{16}$ Therefore,

\footnotetext{
${ }^{16}$ For a comprehensive analysis of a sample of 722 citation contexts citing retracted papers, see research undertaken for this project (Hsiao \& Schneider, 2021).
} 
if researchers do want to properly cite a retracted paper, what should they do? The current recommendation is that authors should (1) note the retraction (e.g., in the article text or citation) and (2) cite both the paper and the retraction notice (Oransky, 2018a). However, meeting the second suggestion is not easy: not all citation styles include guidance for citing retractions. A few citation styles do include clear guidance, as the American Medical Association (AMA) Manual of Style (Christiansen et al., 2020), National Library of Medicine (NLM) Style (Patrias \& Wendling, 2018), and American Psychological Association (APA) Style (American Psychological Association, 2019) do (see Appendix E: Existing Citation Standards for Retracted Publications). However, citation management software often cannot translate relevant bibliographic data (e.g., retraction notice publication date, volume number, page number) into properly formatted reference items in these formats (Suelzer et al., 2019). Improvements in citation style guidelines and citation management software are needed to help authors cite retractions properly and easily.

\section{Visibility of Retraction Status}

The visibility of retraction status should be considered from the publisher's as well as the reader's perspective. From the publisher's perspective, visibility of retraction status can be significantly compromised when indicators of retraction status are inconsistently applied. For example, document types designated for retracted publications, as well as links between retracted publications and their notices, are not present in all cases (Proescholdt \& Schneider, 2020; Schmidt, 2018). Additionally, database search results do not always sufficiently identify an item having been retracted. In one search for a particular author's retracted papers around five months after their retraction, $94 \%$ were identified as retracted in Medline, but only $6 \%$ were identified as retracted in EMBASE (K. Wright \& McDaid, 2011). In the online journals where these articles were published, their status as retracted was only unmistakable in five out of nine cases (K. Wright \& McDaid, 2011). Another study of the Korean medical database KoreaMed showed that many retracted articles lacked watermarks on the journal homepages; however, the presence or absence of a watermark had no apparent effect on the number of times an article was cited postretraction (Kim et al., 2019). Moreover, a discussion paper for our RISRS workshop has drawn attention to the inconsistent display of citations even from the same publisher (Suelzer et al., 2020). To solve this problem, publishers and database providers should reach a consensus regarding how to apply indicators of retraction status and consistently apply such standards throughout their websites and databases, as well as offering notification services for authors to opt into.

However, visibility created by publishers and databases cannot guarantee visibility for users. Many retraction notices are still not freely available, despite COPE guidelines recommendations (Wiedermann, 2018; K. Wright \& McDaid, 2011). Copies of retracted papers in personal libraries and on non-publisher websites may not reflect the retraction status; for instance, if the article was downloaded or incorporated into the library/repository before the retraction date (Davis, 2012). One study found that only a quarter $(26 \%)$ of the retracted articles identified through those resources contained some retraction statement (Davis, 2012). Fortunately, technology has enabled tangible solutions for closing this loophole. For example, Zotero's retraction notification can deliver messages to users about retraction status. CrossMark, a service from CrossRef, 
allows users to retrieve the current status of the paper with a single click. Still, an expansion of retraction notification to all reference management software is necessary, and information literacy such as checking the status of a paper before citing it should be a part of the basic curriculum for researchers. Furthermore, the utility of such tools depends on the accuracy and completeness of the references/citations themselves.

\section{Inconsistent Retraction Metadata}

Consistent and useful metadata related to retraction is necessary to ensure that a researcher can understand the status of a given publication, locate items that have been retracted in a database, and not overlook the fact that a publication they are citing is retracted or may be in the process of investigation. It also enables publishers, database providers, and other stakeholders to implement machine-actionable metadata exchange, and to clearly communicate about retraction statuses. However, a challenge of taxonomy must be addressed before metadata can be consistently applied across stakeholders, as metadata terms related to retraction status (e.g., retraction, erratum, correction, corrected and republished article, retracted and replaced article, withdrawal, etc.) can signify different concepts to different parties. What one publisher refers to as a 'withdrawal' might be called a 'retraction' by another. 'Withdrawal' is a term often applied to preprints, but is sometimes applied in other situations as well. For example, EMBO Press uses 'withdrawal' to refer to author-initiated retractions in the hope that the term carries less stigma than the term 'retraction' (Boxheimer \& Pulverer, 2019; Oransky, 2019). Wiley uses 'withdrawal' to describe situations in which an article has been accepted and received a DOI, but has not yet been formally published; such articles are deleted, but bibliographic information is retained in most cases (Wiley, n.d.). Elsevier uses 'withdrawal' when removing the content of Articles in Press, publications that have been accepted and posted on the publisher website but not formally published (Elsevier, n.d.). When different publishers use terms differently, it can be difficult to understand what the status of a given paper means. While Crossref's Crossmark can help make retraction status more visible, and provides a list of 12 terms to describe various types of updates (Crossref, n.d.), this approach allows publishers to apply terms as they choose, without set definitions for the 12 terms or the relationships between them.

A working group has been formed, led by RISRS workshop participants, to create a taxonomy to help standardize language for referring to retraction status. A presentation at the Society of Scholarly Publishing (SSP), led by former COPE chair Deborah Poff, described the current taxonomy of five terms: Correction, Expression of Concern, Retraction with Replacement, Retraction, and Withdrawal. Removal has been discussed as a possible sixth term, but consensus has not been reached by the group (Flanagin et al., 2021).

Additional metadata fields, such as reasons for retraction, have also been used in the Retraction Watch Database. This metadata may be useful for some purposes, though some workshop participants expressed concerns that a taxonomy that detailed may be too complicated to be broadly adopted across publishers and databases.

Other metadata issues include reuse of a retracted article's DOI for a republication, which can make it difficult for researchers to find the original paper and understand the differences between 
the two. Additionally, within databases, publisher websites, and other sources, the metadata of publications that have been retracted is not always updated to reflect the retracted status. For example, PubMed, Web of Science, and Scopus were all found to contain retracted items not labeled with retraction-related document types, with PubMed containing 58, Web of Science containing 56, and Scopus containing 8654 items that were likely retracted publications or retraction notices, but were not labeled with retraction-related document types (Proescholdt \& Schneider, 2020). Schmidt (2018) previously identified issues with missing retraction-related metadata in PubMed and Web of Science for retracted publications and retraction notices. Inaccuracies in metadata hinder communication and research involving retracted publications.

\section{Quality of Retraction Notices}

How should a retraction notice be written? As of 2019, the COPE guidelines recommend that notices of retraction should: "(1) indicate who is retracting the article; (2) state the reason(s) for retraction; and (3) be objective, factual and avoid inflammatory language" (COPE Council, 2019b). Rules 1 and 2 are also stated in the 2009 version of the COPE guideline for retracting articles (COPE Council, 2009) as well as in the International Committee of Medical Journal Editors (ICMJE) guidelines (International Committee of Medical Journal Editors, 2019). In 2015, Retraction Watch published its own standard, more detailed than that of COPE (Oransky, 2015).

Table 4 summarizes empirical studies on retraction notice quality. Current data suggest inconsistency in the level of compliance with COPE guidelines. Eleven studies covered both reasons for retraction (COPE guideline rule 1 ) and the authority retracting the article (COPE guideline rule 2), five assessed only reasons for retraction, and 1 assessed only the authority retracting the article. These studies found percentages of retraction notices that stated the authority retracting the article that ranged from $24.14-100 \%$ and percentages of retraction notices stating the reasons for retraction that ranged from $62.03-100 \%$ (excluding two outlier studies by Triapthi et al. $(2018,2019)$ that found that only .8\% and $3.6 \%$ provided "explicit" reasons without clarifying whether or not any implicit reasons were given). Note that the studies finding $100 \%$ compliance were focused on narrowly focused datasets, with one studying the works of a particular author (15 notices; Saikia \& Thakuria, 2019), and the other studying only BioMed Central articles from 2000-2015 (134 notices; Moylan \& Kowalczuk, 2016); similarly, the article finding the lowest compliance with rule 2 (stating the retracting authorities) was focused only on Journal Citation Report nursing journals (29 notices; Gray et al., 2019). Moreover, many studies sampled retraction notices over a long period, and the low fraction of retraction notices that do meet the standards might be the result of including historical notices. Only one article reported results based on retraction notices from two single-year periods (2008 vs. 2016) from a single database (PubMed) (Decullier \& Maisonneuve, 2018). In summary, a more systematic assessment of retraction notice quality is needed. Such an assessment should examine retraction notice quality in a more granular time scale, take samples from multiple databases, and better still, evaluate retraction notice quality beyond the minimum requirements of the COPE guidelines. 
Table 4. Retraction notice quality studies and their results

\begin{tabular}{|c|c|c|c|c|}
\hline Reference & $\begin{array}{l}\text { Database/Dataset } \\
\text { studied }\end{array}$ & Period studied & $\begin{array}{l}\text { Fraction of } \\
\text { retraction notices } \\
\text { that stated who is } \\
\text { retracting the } \\
\text { article }\end{array}$ & $\begin{array}{l}\text { Fraction of } \\
\text { retraction notices } \\
\text { that stated the } \\
\text { reason(s) for } \\
\text { retraction }\end{array}$ \\
\hline (N. Wright, 1991) & $\begin{array}{l}\text { Permuterm Subject } \\
\text { Index section of } \\
\text { Science Citation } \\
\text { Index, Medline, } \\
\text { popular literature, } \\
\text { historical surveys, } \\
\text { and news articles }\end{array}$ & 1963-1987 & $89 \%$ & $68 \%$ \\
\hline $\begin{array}{l}\text { (Wager \& Williams, } \\
\text { 2011) }\end{array}$ & Medline & 1980-2009 & $93 \% *$ & $95 \% *$ \\
\hline $\begin{array}{l}\text { (Decullier et al., } \\
\text { 2013) }\end{array}$ & PubMed & 2008 & -- & $91 \%$ \\
\hline $\begin{array}{l}\text { (Balhara \& Mishra, } \\
\text { 2014) }\end{array}$ & $\begin{array}{l}\text { PubMed (articles on } \\
\text { Mental Disorders) }\end{array}$ & $\begin{array}{l}\text { Literature through } 15 \\
\text { September } 2012\end{array}$ & $96.36 \%$ & $85 \%$ * \\
\hline (Bilbrey et al., 2014) & $\begin{array}{l}\text { Selection of higher } \\
\text { and lower impact } \\
\text { journals }\end{array}$ & $\begin{array}{l}1960 \text { to February } \\
2012\end{array}$ & -- & $78 \% *$ \\
\hline (Elia et al., 2014) & Boldt articles & $\begin{array}{l}\text { Through January } \\
2013\end{array}$ & $\begin{array}{l}89.87 \% \text { ** (based on } \\
\text { the } 79 \text { Boldt articles } \\
\text { that had been } \\
\text { retracted at the time } \\
\text { of the study) }\end{array}$ & $\begin{array}{l}62.03 \%^{* *} \text { (based on } \\
\text { the } 79 \text { Boldt articles } \\
\text { that had been } \\
\text { retracted at the time } \\
\text { of the study) }\end{array}$ \\
\hline (Huh et al., 2016) & KoreaMed & $1990-2016$ & $94.7 \%$ & $79.8 \%$ \\
\hline $\begin{array}{l}\text { (Moylan \& } \\
\text { Kowalczuk, 2016) }\end{array}$ & BioMed Central & $2000-2015$ & $94 \% *$ & $\begin{array}{l}100 \% \text { provided } \\
\text { categories of } \\
\text { reasons; } 89 \%{ }^{*} \\
\text { specified whether } \\
\text { the cause was error } \\
\text { or misconduct }\end{array}$ \\
\hline $\begin{array}{l}\text { (Decullier \& } \\
\text { Maisonneuve, 2018) }\end{array}$ & PubMed & 2016 & -- & $\begin{array}{l}99.2 \% \\
\text { However, a } \\
\text { shortcoming is that } \\
16 / 123 \text { withdrawn } \\
\text { items were excluded } \\
\text { (Oransky, 2018b) }\end{array}$ \\
\hline (Tripathi et al., 2018) & Scopus & $2000-2017$ & -- & $3.6 \%$ \\
\hline (Xu \& Hu, 2018) & Web of Science & 1966-2017 & $71.08 \% *$ & -- \\
\hline (Gray et al., 2019) & $\begin{array}{l}29 \text { retracted } \\
\text { manuscripts from } \\
\text { nursing journals } \\
\text { identified by (Al- } \\
\text { Ghareeb et al., } \\
2018 \text { ) }\end{array}$ & $1980-2017$ & $24.14 \%$ ** & $77 \%$ \\
\hline
\end{tabular}




\begin{tabular}{|c|c|c|c|c|}
\hline $\begin{array}{l}\text { (McHugh \& Yentis, } \\
\text { 2019) }\end{array}$ & $\begin{array}{l}\text { Boldt, Fujii, and } \\
\text { Reuben articles }\end{array}$ & -- & $96 \%$ & $94 \%$ \\
\hline $\begin{array}{l}\text { (Saikia \& Thakuria, } \\
\text { 2019) }\end{array}$ & Yuhji Saitoh articles & Through 1 July 2019 & $100 \%$ & $93.3 \%$ \\
\hline (Stavale et al., 2019) & $\begin{array}{l}\text { PubMed, Web of } \\
\text { Science, Brazilian } \\
\text { Virtual Library of } \\
\text { Health (BVS) } \\
\text { databases, Google } \\
\text { Scholar, and } \\
\text { Retraction Watch; } \\
\text { limited to life and } \\
\text { health sciences } \\
\text { articles by authors } \\
\text { with Brazilian } \\
\text { affiliation }\end{array}$ & $2004-2017$ & $97 \% *$ & $93 \%{ }^{*}$ \\
\hline (Tripathi et al., 2019) & Web of Science & 2008-2017 & -- & $0.8 \%$ \\
\hline (Vuong, 2020) & Retraction Watch & $1975-2019$ & $47 \% *$ & $90 \%$ \\
\hline
\end{tabular}

Many authors have drawn attention to the language of retraction notices. Retraction Watch keeps a category called "unhelpful retraction notices," including retraction notices giving reasons for retraction but in opaque language (Marcus, 2018; V. Stern, 2018). A few rhetorical analysis studies also found that authors of retraction notices often use rhetorical strategies (e.g., the use of passive voice and third-person pronouns) that less clearly ascribe responsibility for the events leading to the retraction or the decision to retract to a particular actor (Hesselmann \& Reinhart, 2019; Hu \& Xu, 2020). While detailed and specific information in retraction notices is desirable for readers, editors and publishers are neither omniscient nor unconstrained; further consensus on what is necessary for use of the literature, and what is feasible to provide, will be needed.

\section{Literature Review Conclusions}

Empirical research has exposed a wide range of problems related to the dissemination of retracted papers. Papers continue to be cited after their retraction, and (at least in biomedicine) the predominant majority (>94\%) of post-retraction citations still describe retracted papers as valid work (Hsiao \& Schneider, 2021). The existence of those citations threatens the integrity of the scientific literature and compromises the utility of retraction as a self-correction mechanism for science. Moreover, even when authors find legitimate reasons to cite retracted papers, they face obstacles including the lack of citation guidelines and limited software support. The low visibility of retraction status is often hypothesized to be the cause of unknowing citations. Studies have found low consistency in applying indicators of retraction status across different publisher sites and within the same publisher site. On the other hand, scientists' information behavior should be considered, as problematic citation behavior may contribute to the prevalence of unknowing postretraction citation. Finally, retraction notices should carry crucial information about who is 
retracting a paper, and why, but in some cases this information is missing (Balhara \& Mishra, 2014; Snodgrass \& Pfeifer, 1992; Tripathi et al., 2018). Many retraction notices do not state whether the issues underlying retraction are due to honest error(s) or potential misconduct (Moylan \& Kowalczuk, 2016). Of note, an institutional investigation is needed to make this distinction; it is beyond the remit of journals to adjudicate on intentionality, individual-level responsibility, and/or whether there is evidence of intentional misconduct. Hence, retraction notices can only include such statements in cases where the institution has completed an investigation and relayed its findings to the journal. Guidelines exist for what information a retraction notice should contain (COPE Council, 2019b; International Committee of Medical Journal Editors, 2021). Although many authors in our dataset promote the adherence to COPE guidelines as remedies for many problems, these guidelines have not been consistently followed in practice to date. In addition, retraction notices are often found to be ambiguous and to use rhetorical strategies to shape the perception of responsibility (Hesselmann \& Reinhart, 2019; Souder, 2010). More discussions are needed to reach a consensus among authors, editors, and publishers as to industry-wide standards for the content of retraction notices.

\section{DEFINING PROBLEMS AND OPPORTUNITIES}

The production, communication, circulation, use, and preservation of scholarly materials is defined and organized by the interests of multiple stakeholders. While it is clear that retraction is a complex problem involving multiple aspects of scientific research and scholarly communications, we focus here on the continued citation and use of retracted research. Our research questions focus on the varied stakeholder perceptions of the types of retracted papers, harms associated with retracted research, intervention points to stop the spread of retracted research, the gatekeepers who can disseminate retraction status, and any impediments to the public dissemination of retraction notices. In this way we clarify differences in perception, and identify areas of agreement over the problem definition or perceived solutions.

In this section, we examine problem definitions and linked intervention opportunities from two perspectives. First, we identify perceptions of problems and opportunities in the empirical literature on retraction. Second, we examine stakeholders' perceptions of problems and opportunities based on the stakeholder consultation process. Finally, we conclude this section with comparisons of the most salient problem-definition assumptions we identified in our analysis.

\section{PROBLEMS AND OPPORTUNITIES DESCRIBED BY THE EMPIRICAL LITERATURE ON RETRACTION}

The workshop discussion was framed in part based on the results of a rough thematic analysis, limited to a small set of papers that we had identified in the initial screening stage of our scoping review (covering searches up to April 2020). The RISRS team had identified 162 central papers focused on empirical research on retraction (see Appendix C: Literature Scoping Review Methods and Intermediate Results), and we had already extracted blocks of text related to problems \& opportunities from the discussion and conclusion sections of 143 of these papers in EPPI-Reviewer. About a month before the first workshop, we imported these blocks of textual 
data in the form of coded quotations (one per paper) into Atlas.ti and did a coarse thematic analysis, identifying as many themes per block/paper as relevant (see Appendix B: Problems and Opportunities Dataset and the data itself (Woods, 2021).

From this set of themes (Braun \& Clarke, 2019) we produced a concept map (Conceição et al., 2017; Kane \& Trochim, 2009) of the major problems and opportunities. In each source paper's discussion section, the authors may have framed their discussion in terms of either problems or opportunities or both. We identified and coded the problems that authors associated with the retraction process; attributed to the retraction process; or perceived to be causing retractions. This procedure resulted in 41 distinct codes associated with problems and 38 distinct codes associated with opportunities for addressing the problem of retracted research in the scholarly communications ecosystem.

Since the workshop discussion was framed based on the results of this thematic analysis, we now present it, despite its various limitations. Below, we focus first on the problems related to retracted research and its continued citation and then describe perceived opportunities for addressing these problems, according to the literature sampled.

\section{Problem Themes Described by the Empirical Literature on Retraction}

We used 41 different codes to identify problems associated with retracted research which we clustered into the six problem themes shown in Table 5.

Table 5. Problems

\begin{tabular}{|l|l|}
\hline Problems Themes & Number of Problems \\
\hline Problems associated with Reasons for Retraction & 72 \\
\hline $\begin{array}{l}\text { Problems associated with Procedures, Policies and } \\
\text { Systems }\end{array}$ & 47 \\
\hline Problems associated with Continued Citation & 28 \\
\hline Problems associated with the Visibility of Retraction & 13 \\
\hline Impacts of the Retraction Process & 5 \\
\hline Differences in Institutional Contexts & 5 \\
\hline
\end{tabular}

When sorted by problem theme, the largest number of quotations highlight problems associated with the 'reasons for retraction.' In general this theme concerns assessing the cause of retractions, whether the necessary data for this (generally in retraction notices) exists, or whether reasons for retraction are sufficiently reported. Problems that authors mention within this theme include the categorization of retractions (typically referred in our dataset to as 'reasons for retraction'), the difficulties involved in determining why a paper has been retracted, and whether the reasons for retraction are clear from a retraction notice. Authors in our literature sample were interested in understanding what is driving the rate of retraction (e.g., whether 
misconduct is the primary reason for retraction), yet, due to policies and terminology issues, retraction notices often do not provide sufficiently granular reasons for retraction.

The second largest problem theme concerns how retraction-related problems are inadvertently sustained by current procedures, policies, and systems. One example is the issue of adoption and adherence to COPE and ICMJE guidelines regarding retraction, which is prominently framed both as a problem, related to the broader issue of inconsistent policies, and as a solution to the issue of retracted research and its continued citation. In our data, this is often framed as an issue regarding the efficacy of the COPE and ICMJE standards; the consistency of compliance with COPE recommendations or guidelines; or the standardization of publishing policies more generally. For example, Atlas (2004) comments that, "Few journals have publicly stated policies, most seem to believe it will not happen to them, and, if it does, they will handle it based only on the particulars of the specific incident and not on a well-thought out and wellpublicized policy." Problems of the career system are also discussed, particularly the potential psychological and career harms sustained by 'innocent scientists' in 'costly collaborations' 17 (Mongeon \& Larivière, 2016). Another problem is how easy it seems to be to game the quality assessment system. Markowitz \& Hancock comparatively investigated whether the writing style of papers retracted for fraud differ from non-retracted papers published in the same journal, or for papers retracted for reasons other than fraud "(e.g., ethics violations, authorship issues)". They find that papers retracted for fraud are associated with obfuscated writing: "scientists wrote fraudulent papers with more obfuscation to make them more difficult to assess" (Markowitz \& Hancock, 2016).

Issues related to post-retraction citation follow closely behind these two large problem themes, figuring as a central concern in the literature. Problems in this category are primarily discussed in terms of the persistence of post-retraction citation, the time it takes to correct the literature; and differentiating valid from invalid science at the level of citation. Three observations regarding continued citation stand out. First, "many citations come from citing secondary sources, which would prevent citers from seeing the retraction notice posted on the original article" (Mena et al., 2019). Secondary sources here refer to researchers adopting citations incorporating information and citations from other articles that cite the retracted work. This relates to the second observation, that many citations are copied from the bibliographies of other articles (Hamilton, 2019). Both bring into focus the limitations of efforts to make retractions visible, through watermarking or linked retraction notices. In the age of digital dissemination this is potentially a more vexing problem where "articles even after retraction are continuously being discussed in the social platforms with highest shares on Twitter, Mendeley, blogs, peer-review sites etc." (Jan \& Zainab, 2018). Together, these observations raise the question of how users interact with the citation ecosystem.

\footnotetext{
${ }^{17}$ In "Costly Collaborations: The Impact of Scientific Fraud on Co-Authors' Careers", they write "Scientific fraud in the biomedical field is not only harmful to science as a whole and, at the individual level, to the fraudulent scientist, but also to the innocent scientists whose only fault might have been choosing to work with the wrong colleague." (Mongeon \& Larivière, 2016).
} 
Tightly coupled to this issue of citation behavior, visibility is discussed in the literature as both a problem and a solution to retraction. Many papers report the limits of strategies to make retracted research visible. Poor visibility in turn limits an author's ability to cite retracted research appropriately. For our dataset, where authors discussed retraction as a problem, we saw a major focus on whether readers were aware that research had been retracted prior to citation, or whether a watermark had been used to denote retraction status.

The impact of the retraction process is also a topic of concern, with a number of papers framing the issue of impact in terms of career stigma, as well as financial impacts. Finally, differences in institutional contexts encompasses variations in standards and in resources. An overriding concern in this problem area is the question of whether institutions have sufficiently developed capacities for ethics and integrity training.

\section{Opportunity Themes Described by the Empirical Literature on Retraction}

From the above set of 143 papers, 38 separate codes were associated with opportunities for addressing or resolving issues related to retractions and/or the continued citation of retracted research. These separate codes can be similarly clustered into six discrete 'opportunity themes' as suggested by the papers' authors (see Table 6).

Table 6. Opportunity Themes

\begin{tabular}{|l|l|}
\hline Opportunity Themes & Number of Opportunities \\
\hline Implementing Stakeholder Reforms & 161 \\
\hline Adopting Review Strategies & 47 \\
\hline Refining Retraction Classification & 27 \\
\hline Innovating Visibility Strategies & 24 \\
\hline Addressing Research Integrity Issues & 19 \\
\hline Further Research & 5 \\
\hline
\end{tabular}

The largest of these opportunity themes broadly concerned implementing stakeholder reforms to target how researchers, publishers and editors, libraries, and professional associations manage retracted research in the scholarly communications ecosystem. In our literature sample, the greatest number of reform propositions in the discussion sections were coded as targeting reform of publishing practice. By contrast a smaller number of the codes in our dataset focused on reforming how scholars do scholarship, and on mobilizing specific stakeholders to influence change (see Table 7). 
Table 7. Areas of Stakeholder Reform

\begin{tabular}{|l|l|}
\hline Area of Stakeholder Reform & Number of Proposals \\
\hline Reforming Publishing Practice & 70 \\
\hline Reforming Scholarly Practice & 33 \\
\hline Mobilizing Stakeholder Influence & 33 \\
\hline Educating Stakeholders & 25 \\
\hline
\end{tabular}

The reform of publishing practice refers to proposals that target industry-wide reforms of the publishing industry. These reforms consist of the publishing industry's adherence to the COPE and ICMJE standards; the implementation of industry-wide checklists; standardized editorial policies; cross-industry quality control guidelines; and generalized industry policies on reporting to repositories and other venues; and standards regarding retraction notice quality, including detailed consistent reasons for retraction, or the development of a standardized retraction form. In some instances, these suggestions are couched in generalized appeals to "universal publishing standards to minimize the continuing citation of retracted articles as valid work," (Wasiak et al., 2018), including adoption of standards to promote "transparency and openness in scientific communication," (Wasiak et al., 2018) or "universally accepted standards for unambiguous retraction" (Wiedermann, 2018).

In the 143 papers reviewed for this analysis, proposals to reform scholarly practice consist of ideas to change how science can incorporate higher standards of transparency, clear norms regarding misconduct, and stronger standards of replication and reproducibility. This includes the need for researchers and authors taking care to steward their own bibliographies and reference lists ${ }^{18}$ (Davis, 2015; Kochan, 1992; Hamilton 2019) as well as to pay greater attention to the risks associated with misconduct, such as fraud (Foo, 2011). In regards to transparency, some advise that the proprietary attitude of academics toward data should be "superseded by an obligation to demonstrate ethical research practices by lodging data in a way that facilitates inspection, reanalysis, and replication" (Tourish \& Craig, 2020). Reform strategies also propose new approaches to how science is conducted. Trikalinos suggests that while "Trust is fundamental for scientific progress," the scientific community should encourage "the careful, rigorous replication of research findings by other teams" (Trikalinos et al., 2008). The reform of research culture also includes naturalizing the correction of error in the scholarly record, where "research mistakes, like all human errors, must be seen not as sources of embarrassment or failure, but rather as opportunities for learning and improvement" (Nath et al., 2006).

\footnotetext{
${ }^{18}$ In research undertaken for this project, we found that retracted papers can be cited for reasonable intents. For example, systematic reviews may cite retracted papers in order to provide an exclusion rationale for excluding papers from the analysis. Papers following up on real-world impacts attributed to retracted research cited the retracted papers to provide background information (e.g., Wakefield's influence on the anti-vaccine movement). However, in our dataset, taken from biomedicine, only $5.4 \%$ of post-retraction citation contexts (722 in 13,252 citation contexts) documented the retraction, which suggests that the the findings reported in the retracted papers may be improperly spread through the vast majority of their post-retraction citations (Hsiao \& Schneider, 2021).
} 
Some authors suggest broad measures that cut across stakeholder groups. Wasiak et al., for example, highlight a coordinating nexus of stakeholder reform strategies that involve "support for a stand-alone retraction studies database" coupled with "authors signing compulsory declaration forms that detail antiplagiarism guidelines," and for publishers to adopt "guidelines that support standards in transparency and openness in scientific communication" (Wasiak et al., 2018). Others emphasize a coordinated vigilance on the part of "reviewers, editors, and readers," to report suspect citations and coordinated "investigations by institutions, government agencies, and journalists in identifying and documenting research misconduct," (Fang et al., 2012).

The next largest theme focuses on the adoption of various review strategies to weed out papers before they need to be retracted. These include automated review procedures to detect plagiarism by checking publications at the time of submission for text overlap. A similar proposal suggests publishers also scan an article's bibliography to detect retracted citations as well. Some of these proposals include incorporating Crossmark (Crossref, n.d.), while others suggest that editors and researchers should frequently check Retraction Watch (Retraction Watch, n.d.d) to monitor for retracted research and to review citations employed in their papers.

A related body of proposals focus on refining the retraction taxonomies used in retraction notices, and in innovating new strategies to enhance retraction visibility. The proposals in each set are similar to those identified in the problem themes that highlight retraction visibility, and retraction notice granularity. One proposed solution is the adoption of standardized retraction categories to be incorporated into publishing workflows that can be used to denote the type of amendment made to the article, and the reason for retraction. For example, Moylan \& Kowalczuk note that in order to improve transparency, "publishers could enforce the use of an internal checklist capturing the main information required in a retraction notice along with template wording" to help standardize the retraction notice (Moylan \& Kowalczuk, 2016). This issue of a common retraction taxonomy is linked to the issue of visibility, where many argue that "it is essential to make the retracted articles transparent, visible and clear to readers in order to avoid post retraction citation," (Bolboacă et al., 2019). Additionally, some argue for "a prominent placement of the word 'retraction' on the first page" of an article, noting that once an article is downloaded, retraction notices are often "left behind" (Neale et al., 2007).

Text coded as 'addressing research integrity issues' refers to reforms that would support research institutions in appropriately addressing research misconduct; creating more money and incentives for research integrity training; and incentivizing editors and researchers to adopt more stringent standards for research integrity.

A small body of texts from our 143 papers suggests areas for further research on retraction. Balhara (2014) suggests researchers should study the impact of open access retraction notice dissemination, whereas Steen (2013) encourages more research on the cause of retractions to inform culture change and address the loss of trust in science. Stern (2014) suggests further empirical study of misconduct. By contrast, Chen (2013) suggests that routine preventative 
monitoring of the scientific literature would help identify articles to be retracted, as well as closely-related articles, and argues for the development of new tools to help verify the integrity of citation genealogies. ${ }^{19}$ In a similar vein, Korpela (2010) argues for software tools to help "authors, reviewers and editors to check the list of references free of charge for a manuscript submitted for publication" against retracted publication lists and for searching for misconduct that has not been retracted based on "author names of fully investigated and convicted scientific misconduct cases."

\section{Problem Definition Described by the Empirical Literature on Retraction}

Two problem themes stand out as shown in Table 8: misconduct as a reason for retraction and reasons for retraction (in general). Misconduct as a reason for retraction speaks to two concerns: the question of what is driving retraction and the quality of information reported in retraction notices.

Table 8. Most-discussed individual problems

\begin{tabular}{|l|l|l|}
\hline Problem Themes & Problem & Number of Proposals \\
\hline $\begin{array}{l}\text { Problems with Reasons } \\
\text { for Retraction }\end{array}$ & $\begin{array}{l}\text { Misconduct as a Reason } \\
\text { for Retraction }\end{array}$ & 33 \\
\hline $\begin{array}{l}\text { Problems with Reasons } \\
\text { for Retraction }\end{array}$ & Reasons for Retraction & 26 \\
\hline
\end{tabular}

Likewise, two opportunity themes stand out as shown in Table 9: guidelines and policies within the publishing industry and clear reasons for retraction. Authors suggest clarifying how an article has been amended and why, in order to enable readers evaluate whether a work has been invalidated, and in order to assist readers in "recognizing retracted literature and avoiding inappropriate citation," (Fulton et al., 2015) as well as send a clear message regarding the consequences of publishing falsified or fraudulent material (Cox et al., 2018).

Table 9. Most-discussed individual opportunities

\begin{tabular}{|l|l|l|}
\hline Opportunity Themes & Opportunity Pathway & Number of Proposals \\
\hline Publishing Industry & $\begin{array}{l}\text { Guidelines and Policies } \\
\text { within the Publishing } \\
\text { Industry }\end{array}$ & 24 \\
\hline Publishing Industry & $\begin{array}{l}\text { Clear Reasons for } \\
\text { Retraction }\end{array}$ & 23 \\
\hline
\end{tabular}

\footnotetext{
${ }^{19}$ In research for this project, we created the keystone citation framework to identify when a paper fundamentally depends on work it cites (Fu \& Schneider, 2020). This allows tracing the logical dependencies of retracted and other problematic science. In work in progress we are exploring how to automate detection of methods-related keystone citations (Fu et al., 2021).
} 
Comparing the problem and opportunity themes, we noticed an overlap in the codes: the most frequent codes concern reasons for retraction and guidelines and policies for retraction notices.

\section{THEMES FROM STAKEHOLDER INTERVIEWS}

In the consultation process, stakeholders from across the scholarly communications ecosystem were invited to participate in semi-structured interviews (see Appendix D: Stakeholder Consultation Process). Here, we review problems and opportunities from these stakeholder interviews, along with broader themes relevant to issues of implementation.

\section{Problem Frameworks based on Stakeholder Interviews}

From stakeholder consultation interviews, we identified three problem frameworks. These are background assumptions that shape the scale and scope of how the issue of retracted research is defined as a problem and subsequently evaluated and framed by stakeholders. These are the 'risk' framework, the 'self-correction' framework, and the 'wrong-doing' framework. The 'risk' framework concerns how stakeholders frame stigma and evaluate the risks associated with retracted research, whereas the 'self-correction' and 'wrong-doing' frameworks refer to the background assumptions stakeholders adopt when discussing the relationship between retraction and science as a social institution or cultural practice.

The 'risk' framework concerns stakeholders' orientation to the risks associated with retracted research, often framed in terms of stigma. We see this, for example, in the impact that retraction processes have on multiple stakeholder communities. Researchers, for example, face career impacts by way of reputational damage. Editors likewise report issues of stigma, and a reluctance to engage in retraction processes due to career and reputational impacts as well as fear of litigation. Here, the harms associated with retraction include reputational damage, lack of support from their publishers, and fear of the hostility associated with uncooperative and potentially litigious researchers. Many stakeholders additionally report emotional tolls that speak to broader personal impacts, such as psychological issues and professional and personal relationship stress.

The 'self-correction' framework concerns the implications of retraction for the conduct and communication of science. Here, one assumes science is a 'self-correcting system' and the role of retraction, coupled with other interventions, such as corrections, is to clean up errors in the literature. Many stakeholders viewed the prevalence of retracted science as an indicator of the health of scientific publishing - that the system was in fact working to clean up the literature. To some degree, this assumption is reflected in the literature (Fanelli, 2013), but we also found it to be prevalent throughout the stakeholder consultation process. There is some question of whether the notion of science as a self-correcting system is inclusive of non-scientific scholarship. Is it useful to view all research as self-correcting?

This assumption is usefully contrasted with a second background assumption - the 'wrongdoing' framework-in which stakeholders assume that the prevalence of retractions is best understood as a failure of scientific practice, and hence, a problem with either the methods 
utilized, or the conduct of individual scientists or scholars. The continued citation and use of retracted research are best addressed, on this body of assumptions, through repair of the methods used to conduct, review, and evaluate science. Incentive systems based on speed and numbers of papers published are an environmental pressure that shapes all of these methods.

Both the 'self-correcting' and the 'wrong-doing' frameworks hold direct implications for how we understand the purpose of retraction as well as how we evaluate solutions to the continued citation of retracted research.

For stakeholders who view retraction as part of science as a self-correcting process (e.g., primarily as a tool to clean up the scientific or scholarly record), the issue of retracted research and its continued citation and use is best addressed through optimizing how we communicate retractions and other post-publication amendments; through retraction notices, visual display, and standardized categories of amendment types (e.g., correction, retraction, expression of concern).

Yet for those stakeholders who view retraction primarily as an outcome of either flawed research or scientific misconduct, the primary issue is not how retracted science is communicated, but rather how to keep invalidated science from polluting the scholarly literature or scientific record. For some of our interviewees, this means removing retracted research from the literature to prevent citation, i.e. that retracted research should under no circumstances be cited. By contrast, other interviewees suggested finding a way to sequester retracted publications in order to clearly distinguish retracted publications from the current best evidence. Under a similar set of assumptions some interviewees suggested that the continued citation of retracted research is best addressed through broader reform measures that emphasize the period before flawed research enters the scholarly record. Interviewees also suggest keeping retracted publications available for inspection (e.g., for ethics-related research), but removing retracted publications from everyday information retrieval systems used in evidence synthesis and domain research. Recommendations in this vein include methodological and ethical reforms, but also reforming how researchers learn to publish, navigate peer review, etc. Other recommendations focus on the reform of peer review, issues of reproducibility, or more generally, the importance of addressing the integrity of research before it is communicated, and hence before it might be cited.

Finally, the continued citation of retracted science is often treated as a type of misinformation. Treating retraction as misinformation holds implications for the public nature of science, and questions regarding the public trust in science. In a more limited sense, the circulation of scientific misinformation, 'fake science' and disproven science is particularly concerning for the use of scientific evidence in a variety of regulatory processes.

Many interviewees are concerned specifically with the loss of trust in science and scholarship. This includes concern with the problem of how to address the destabilizing effects of retraction on the use of scientific evidence in regulatory and governance processes. Linked to this concern, some interviewees wanted the public to understand 'science as a self-correcting 
process'. Likewise, in interviews and during the workshop, a number of stakeholders were keen to address the growing circulation and public reception of scholarly preprints.

\section{Contentious Themes based on Stakeholder Interviews}

In RISRS interviews, stakeholders described a range of assumptions that could be glossed over as 'semantic differences.' In the weakest sense, these differences are active as unspoken premises for framing problems and opportunities. In a stronger sense, they may also function as implicit agreements or disagreements over the meaning and scope of what it means to amending the scholarly record. These 'contentious themes' consist of differences of opinion over the purpose of retraction, how to incorporate changes to the scholarly record, the impacts or harms associated with retraction, and the character of reform among stakeholder groups.

\section{The Purpose of Retraction According to Stakeholder Interviews}

In the previous section, we reviewed three broad background assumptions regarding the role of retraction in scholarly publishing, the 'risk', 'self-correcting' and 'misconduct' frameworks. These frameworks influence how stakeholders understand the meaning and scope of retraction in practice.

From our interviews it became clear that the definition of retraction is not commonly held across all stakeholder groups. Interviewees often used similar vocabulary, but with different meanings, or used editorial nomenclature to differing ends. For example, some suggested that retraction only applies to peer reviewed literature, whereas others extended the term to cover preprints. In the latter case the sense of the term is used similarly to 'recalled.'

This variation applies as well to the general purpose attributed to the retraction process which informs editorial decision-making. Retraction is categorized according to the reasons for retraction and/or the source of retraction. Reasons may include classes of scientific offenses such as fabrication, falsification, plagiarism, or gross error. Sources may include authors selfretracting, editors initiating a retraction, or third parties-such as research institutions, funders or government agencies-proposing retraction. In determining whether to retract or to use some other form of post-publication amendment, many interviewees used almost philosophical terms: Who is the legitimate retractor? Who needs to agree with the retraction, the editor, author and peer reviewers? What happens if these actors disagree? Who has the final word? It became clear in our conversations that while there is general guidance regarding many of these questions that guidance is also unevenly distributed throughout the scholarly publishing ecosystem. ${ }^{20}$

${ }^{20} \mathrm{COPE}$ answers these questions clearly, indicating that the editor has final responsibility: "In some cases, retractions are issued jointly or on behalf of the journal's owner (eg, a learned society or publisher). However, since responsibility for the journal's content rests with the editor, they should always have the final decision about retracting material. Editors may retract publications (or issue expressions of concern) even if all or some of the authors do not agree. Who is retracting the article should be clearly identified within the retraction notice." (COPE Council, 2019b) 
The definition of retraction shapes how stakeholders understand the role of retraction in scholarly publishing, particularly in terms of what a retraction is meant to communicate. Some strongly suggest that retraction of a paper is so severe that the paper should never be cited. Others suggest that retraction falls on a continuum of post-publication changes to the scholarly record.

\section{Changing the Scholarly Record}

Preserving the integrity of the scholarly record is seen by some as a valued end in itself. Interviewees recognize that responsibility for assuring the quality and integrity of the scholarly record is distributed amongst librarians, researchers, and those in the publishing industry. Additionally, interviewees broadly agree that it is urgent to come to a consensus agreement about how to incorporate changes to the record. In practice, this is often framed in terms of tools to communicate a paper's status, and to help researchers, editors and decision-makers evaluate research integrity, relevance, and quality.

However, in our interviews the value of retracted research within the scientific record is contested. In some instances interviewees discussed retraction as a sign of health-that the rate of retraction means science is working, and that publishers and editors are doing their job stewarding the scholarly record. Retraction notices with robust reasons add value to the scholarly record, allowing for nuanced evaluation and assessment of retracted material, and intentional citation of retracted work, where warranted, with awareness of the reasons for retraction. Accordingly, some interviewees suggested that there is a value to 'negative knowledge,' or knowledge of previous error, or of what should be professionally avoided. This perspective places a premium on transparency to suggest retractions should be made visible but retained as a matter of record, emphasizing the educational role of such cases. This broadly accords with the notion that science is a self-correcting system.

Others suggest, by contrast, that the value communicated by retractions is limited, and retractions are either best sequestered, or removed from the literature entirely. This aligns more with the sense of retraction as 'wrong-doing,' as was discussed above. On this account retraction is taken as a sign that the scholarly process does not work.

All of these themes concern how retraction and post-publication amendments are incorporated into the scholarly record. Some interviewees desire robust, transparent retraction notices that would allow for robust and nuanced citation practices. Others, by contrast, think that retracted research should quite literally be removed from the scholarly record entirely, as if the compromised research never took place. Interviewees in this camp have proposed, for purposes of research involving retraction, that there be a central, specialized database that quarantines retracted research, so as not to contravene the work of evidence synthesis.

\section{The Harms of Retraction According to Stakeholder Interviews}

Interviewees broadly characterize the harms associated with retraction and continued citation of retracted research as distributed amongst a variety of stakeholders, but generally frame harms in terms of impact, primarily clustering around those associated with decision-making that relies 
on invalid information; on wasted resources, characterized in terms of time, scientific labor and money; and the harm to science resulting from a betrayal of public trust. In these examples, the greater harm is to the prospect and reliability of scientific or scholarly research.

Interviewees framed additional harms in terms of the emotional impact of retraction on researchers and editors, where the retraction process, and science more generally, is characterized as a very 'human,' fallible, prone to mistakes, and conflicted. These emotional impacts are often framed as impediments to retraction; as well as evidence that the system must be fixed, particularly if we are to see retraction and other post-publication amendments as aspects of science as a 'self-correcting' system.

Research and its communication are subject to mixed motives, where editors and researchers' interests are shaped by pressures to publish, the pursuit of prestige and profit, and by legal constraints. These qualities impede efforts at transparent communication, perhaps preventing editors, researchers, and institutions from cooperating, and complicating a process frequently characterized as both resource intensive and time-consuming. Interviewees also suggested that retraction is also emotionally taxing for both authors and editors, as well as for their professional peers and social circles.

\section{The Character of Reform According to Stakeholder Interviews}

While opinions regarding the necessity of reform differ, there is strong agreement among our interviewees that the culture of research and scientific publishing must change. Salient examples of cultural change include reforming citation practice, instituting broad changes to the education of researchers and editors, and redefining how retraction is both stigmatized and valued amongst researchers and within institutional settings.

Proposals to reform citation practice suggest that not all retracted research should be discarded, but rather incorporated through nuanced citations that denote why the retraction occurred, how the retracted research should be evaluated for particular purposes, and whether citation is affirmative or critical. For example, is a cited work acknowledging the retracted research, for purposes of scholarship, or is it citing the study as an example, ignorant of the problems with the research? In cases of post-publication changes, such as retraction with re-publication, is there a way to distinguish between versions within the citation, or, for example, to cite aspects of a particular study that has been corrected? These issues of citation behavior not only hold implications for reforming impact factor schemes along such nuanced formats, but also for how reference managers and citation review technologies are adopted as part of citation behavior.

Although retraction for honest error is a possibility, in interviews retraction was also strongly associated with concerns about misconduct. To create positive incentives for engaging with retraction as a naturalized feature of science rather than a pathology, many propose creating new incentives for valuing retraction as a feature of a researcher's conduct as well as a journal's prestige. 
Interviewees proposed new educational programs to train researchers and editors to understand the retraction process and evaluate retracted research. Often, these proposals assume that retraction is a basic feature of science as a self-correcting system. Others suggest that education is needed to reform peer review, to teach researchers about the consequences of retraction, or to promote post-publication review. This coincides with proposed reforms designed to change how retraction is penalized, or whether it should be rewarded in some cases.

\section{SYNTHESIZING THE PROBLEMS AND OPPORTUNITIES}

\section{Aligning Opportunity Pathways}

We see the continued citation and use of retracted research as an ecosystem problem that cannot be solved by any single stakeholder group working alone. Rather, broad cooperation between stakeholder groups is needed to produce standards, agreements, and technologies to reduce the spread of retracted research in practice. Multiple forms of collaboration and cooperation amongst various stakeholder groups will be needed to identify common norms, standardize responsibilities, and align incentives. This work is threatened by contending definitions of the problem or if stakeholders face disincentives for participating in retraction processes, as well as regional differences in perceptions.

In this context, understanding what ought to be done to address retraction and identifying recommendations that will be successfully adopted by stakeholders rests in part on broad agreement about what is assumed to be possible. For example, as noted above, in the consultation process we identified a tension between those who favored the systematic reform of science and/or the scientific publishing industry and those who favored targeted, incremental reforms. Those that see the need for systemic reform tend to favor solutions premised on radical changes to the structure and practice of scientific publishing. By contrast, those who favor incremental change prefer mechanisms such as standards development or adherence to cross-industry policies.

Questions of stigma and reputational impact may seem to detract from the central concern of this project and its focus on the continued circulation of retracted research. Indeed, throughout the project it has been something of a fulcrum, where, in the opinion of some stakeholders, focusing on the reputational threat seems misguided. However, the issue of stigma and associated impacts illustrates the outsized impact of retracted research as a nested problem. Stakeholders who associate retraction with stigma and costs to career, reputation, and personal well-being, may be reluctant to adopt and implement broad reforms that strengthen the retraction process, even for issues of honest error.

In both the literature review and stakeholder interviews, we identified discrete pathways to address the continued spread of retracted research. These pathways were often characterized in terms of technological innovation. One, frequently noted, is technology to scan bibliographies for retracted research during submission of an article for publication, during the peer review process, or as an aspect of the quality assurance pipeline. Another is a granular metadata 
scheme for post-publication amendments that categorizes items based on reasons for retraction ${ }^{21}$, and a more sustained effort to include bidirectional links between retracted papers and retraction notices. Additional suggestions focused on the role of reference management software in notifying researchers when articles have been retracted.

However, these proposed solutions are often coupled with acknowledged barriers to implementation. Reference checks add more work to an already taxed publishing system. Granular metadata and bidirectional linkages face cooperation hurdles between multiple sectors of the publishing ecosystem and can be costly to develop and implement. Reference management programs are unevenly used by busy researchers whose reference collections are often treated as aggregative.

In addition to these barriers to implementation, we have also identified disagreement over the purpose, scope, and ownership of both common agreements and ecosystem-wide standards. Where guidelines have been developed, stakeholders acknowledge adherence gaps. These gaps cluster around lack of common definitions, uneven implementation of existing standards, and divergent retraction policies at publishing houses, all of which create impediments to communication and collaboration.

Additionally, interviewees suggested that powerful stakeholders, such as publishers or universities, manipulate these gaps to their advantage, perhaps creating disincentives to widespread change or collaboration. To counter these dynamics, some interviewees stressed the urgency of developing or aligning incentives for stakeholders to grapple with the continued spread of retracted research. Such incentives might come from adoption of stronger standards amongst stakeholders or creation of a centralized authority to create or enforce norms.

\section{Defining the Scale and Scope of the Problem}

Mitigating the effects of the continued spread of retracted research will require strategies that involve multiple types of coordinated interventions. Multiple stakeholder communities and multiple areas of the scholarly publishing ecosystem are involved, and stakeholder groups are impacted by retracted research in differing ways. In our discussion above we highlighted a number of ways that the citation and use of retracted research can be addressed, according to a variety of stakeholders. In some cases, stakeholders have suggested that what is needed is simply to disseminate and adopt existing practices, whereas in other cases, we identified innovation problems that will require the development of new standards, policies, or norms. In addition to these issues, there is the persistent system-level issue of how to incentivize change and build the will to address recognized issues in collaborative ways.

All of the problem and opportunity themes discussed above, and the proposed problem definitions, were vetted and discussed by stakeholders in the workshop process. Many of the

\footnotetext{
21 There is tension in determining the appropriate level of granularity: for applying a taxonomy, publishers call for a small list of simple-to-apply categories whereas for using items categorized with a taxonomy, researchers often seek detailed reasons for retraction.
} 
recommendations below take up the overlapping problem definition regarding standards for retraction categorization and visibility of retracted materials, to suggest recommendations in several areas within the scholarly communications ecosystem. The RISRS team feels there is strong agreement in both problem assessment and diagnosis amongst the stakeholders and the literature that might be enhanced through further targeted work. Below, we give some recommendations on what areas to target, the implementation actions that can be taken to build on each recommendation, and spaces where further research can support the refinement of the proposed implementation areas. 


\section{RECOMMENDATIONS}

Recommendations refer to cross-sectoral areas where action can be taken to address retracted research and its continued citation. Rather than target a particular sector, or problem, the recommendations below speak to multiple points in the scholarly communications ecosystem.

Here, we prioritize recommendations for which there exists momentum to address the issue; known examples that can be used to model standards or best practices; current technologies that can be adopted; and proposals for which there is existing or strong agreement.

\section{Develop a Systematic Cross-industry Approach to Ensure the Public Availability of Consistent, Standardized, Interoperable, and Timely Information about Retractions.}

Over $94 \%$ of post-retraction citations in biomedicine do not demonstrate awareness that the cited item was retracted (Hsiao \& Schneider, 2021). Users' typical citation workflows may involve citing preprints, reusing downloaded PDFs, citing older works contained in their reference managers, and copying citations from their own or others' previous bibliographies (Bar-llan \& Halevi, 2018; Davis, 2012). Among citation styles, only the American Medical Association (Christiansen et al., 2020), National Library of Medicine (Patrias \& Wendling, 2018), and American Psychological Association (2019) styles provide explicit standards for citing retracted papers (See Appendix E: Existing Citation Standards for Retracted Publications). Among commonly used systems, only a handful of databases (such as PubMed and RetractionWatch) and tools built on them (such as Zotero, RedacTek, and scite) ensure that users know that a paper they are citing is retracted.

Information about retraction needs to move across different industry information providers (publishers, abstracting and indexing services, scholarly search engines, etc.). However, currently this need is challenged by non-robust dissemination, inconsistent information, and inconsistent presentation of retraction status information (Schmidt, 2018; Suelzer et al., 2020, 2021; K. Wright \& McDaid, 2011).

Shared standards amongst publishers are necessary, but currently there are no industry-wide standards for retraction information or its visibility. The best existing guidelines, from COPE (COPE Council, 2019b) and the ICMJE (International Committee of Medical Journal Editors, 2019), recommend how to make retraction information easy to use and find. However, they are not uniformly adopted. Although both are widely accepted by many publishing groups, particularly in medicine (COPE Council, n.d.; International Committee of Medical Journal Editors, 2021), previous research has found that publishers do not uniformly adhere to COPE and ICMJE recommendations (Balhara \& Mishra, 2014; Bilbrey et al., 2014; Snodgrass \& Pfeifer, 1992). Additionally, more consistent display standards are needed, particularly regarding uniformity in landing pages (Suelzer et al., 2020). 


\section{Individual Recommendations for Consistent, Standardized, Interoperable, and Timely Information about Retractions}

1. Support and motivate stakeholders to consistently adopt and follow the recommendations from COPE and the ICMJE for managing retracted articles and retraction notices.

2. Organize a working group to define best practices addressing retraction and post publication amendments in citation styles and citation software. Membership could be drawn from COPE, NISO, and stakeholders such as publishing house production teams, journal editors, authors of writing style manuals, reference management software developers, and the maintainers of Citation Style Language.

a. Develop additional citation styles and standards for indicating the retraction or correction status of a paper in text and in a bibliography. ${ }^{22}$

b. Advocate for researchers to adopt and use citation software that flags retracted papers. (Zotero is a current example of such software, in partnership with Retraction Watch data.)

c. Advocate for the adoption by publishers of title update procedures for retracted articles to include 'Retracted' directly in the title.

3. Codify best practices and develop sustainable funding sources for databases to facilitate the public and unrestricted access to and dissemination of retraction notices. At minimum, databases should feature Application Programming Interfaces (APIs) to track and disseminate retraction statuses.

a. Example - limited to biomedicine: PubMed is a public database with an API that allows for unrestricted access to and dissemination of retraction notices, but only in biomedicine. It is free to users, and is completely funded by the United States government.

b. Example - restricted access: Retraction Watch is a public database with restricted access. Free public results are limited to 600. It does not have an API as of July 2021. License agreements are required for bulk use. Its funding has included grants, private donations, and licensing agreements.

4. Support resources and standards to maintain databases that facilitate the public and unrestricted access to and dissemination of retraction statuses and retraction notices.

a. Develop processes, agreements, and standards for retraction data interchange between publishers, aggregators, and database providers.

b. Advocate for improvements to existing software (e.g., EndNote, Mendeley, Paperpile, RefWorks, etc.) to flag retracted papers. Retraction Watch is a licensable source of good quality, domain-independent data about retracted papers.

c. Develop model license agreements for use between publishers and aggregators to make information flow. Expand on established agreements such as the

${ }^{22}$ Some current standards Appendix E: Existing Citation Standards for Retracted Publications 
National Library of Medicine's participation agreement for deposit (U.S. National Library of Medicine, n.d.).

d. In implementing processes and standards for data interchange, develop automatic processes that simplify the process of systematically disseminating and integrating updates, prioritizing interoperability across metadata services and providers throughout the scholarly communications ecosystem.

5. Encourage publishers to support or adopt software solutions that enable systematic identification of retracted articles in bibliographies prior to publication. Advocate for the integration of such tools into reference and submission management platforms for easy access and utilization by publishers.

a. Adopt tools for checking bibliographies for retracted paper as part of manuscript review and publishing workflows

i. Example: scite's Reference Check, integrated into Aries' Editorial Manager, Akron ApS's Manuscript Manager, and ScholarOne. (Currently scite checks against the Retraction Watch Database, but after September 2021, will only check for retractions listed in Crossref and/or PubMed.)

ii. Example: eXstyles and Edifix (only retractions listed in Crossref and/or PubMed).

b. Advocate for publishers to adopt Crossmark (Crossref, n.d.) and to invest in maintaining content, including promptly registering updates with the Crossmark data service. Crossmark became free to Crossref members in March 2020 (Meddings, 2020).

\section{Recommend a Taxonomy of Retraction Categories/Classifications and Corresponding Retraction Metadata that can be Adopted by All Stakeholders.}

Retraction notices often provide vague or limited information about the reasons for retraction (Balhara \& Mishra, 2014; Budd et al., 1998; Moylan \& Kowalczuk, 2016). People using retracted science and evaluating authors of retracted science demand additional context about retraction to both clean up the literature and to disincentivize misconduct (Wager \& Williams, 2011). Concerns about possible reputational damage and the risk of litigation can disincentivize the use of more fine-grained distinctions about reasons for retraction (Tourish \& Craig, 2020).

\section{Individual Recommendations for Taxonomy and Metadata}

1. Develop a taxonomy of retraction categories and corresponding metadata standards in tandem.

a. Host, curate, and maintain the taxonomy and metadata standards on a discoverable website with a formal home, based in an industry standards organization such as NISO or the International Association of Scientific, Technical and Medical Publishers (STM).

b. Provide both human-readable and machine-readable metadata.

c. Build support and influence via adoption by highly visible organizations that will endorse and adopt the taxonomy and metadata standards, then support and motivate other stakeholders to adopt them. 
d. Integrate the taxonomy of retraction categories into existing versioning systems (e.g., for DOI incrementing, Crossmark amendments, etc.).

e. Recommend how database records for retractions should include the taxonomy term, when a taxonomy is implemented and adopted by journals.

f. Identify additional areas for future taxonomies or nomenclature standardization.

2. Support the use of persistent identifiers throughout the publishing ecosystem to enable audit-like functions to track amendments (Barbour et al., 2017). Build off of the F1000 model: "All versions of an article are accessible, each with their own DOI (digital object identifier) and may be cited individually. The most recent article version is displayed as the default, and older article versions display a clear notification that newer versions are available." (F1000Research, n.d.)

\section{Develop Best Practices for Coordinating the Retraction Process to Enable Timely, Fair, and Unbiased Outcomes.}

The time between the publication of papers and their potential amendment or retraction is a period in which papers may be adopted, used, and woven into the tapestry of scholarship. This time has been as long as 45 years (PMID:1233443), but papers retracted quickly may also receive fewer post-retraction citations (Hsiao \& Schneider, 2021). Reducing the time to retraction is desirable to ensure the clear and timely communication of amendments to publications. Another danger is that compromised research is identified but fails to be retracted because of logistical complexity amongst all stakeholders involved in the retraction process. In these cases, failure to retract enables the continued citation of research that should have been retracted.

Existing guidelines acknowledge the problems related to time to retraction. For example, the COPE 2019 guidelines say: "Publications should be retracted as soon as possible after the editor is convinced that the publication is seriously flawed, misleading, or falls into any of the categories described above." However, stakeholders suggest the complexity of coordination amongst authors, co-authors, editors, and in some cases institutions may present complex logistical problems or conflicts of interest. For example, review of compromised figures, datasets, and data represented in images can be costly and time consuming. For editors and publishers, the COPE flowchart library is in common use, and could be a model for developing workflow models and suggestions aimed at a variety of additional stakeholders. Some suggest that efforts to innovate retraction processes in this nexus-between institutions, publishers/editors, funders and researchers/editors-are often hampered by perceptions of risk and liability. Early adopters of reforms potentially face increased risks (e.g., liability) on top of the cost of developing policies and procedures; potential costs include referral boards or independent investigative bodies. 


\section{Individual Recommendations for Coordinating the Retraction Process}

1. Use the Cooperation \& Liaison between Universities \& Editors (CLUE) report (Wager et al., 2021) recommendations to develop best practice guidelines to streamline the retraction process with respect to institutions and sponsoring agencies by improving coordination between institutions, publishers, funders and researchers.

2. Clarify best practices and guidelines for journals, authors, and institutions to efficiently coordinate and address concerns about published work.

a. Offer fast-tracks for retraction notices to move through the process more quickly, if the authors agree with or request retraction, or if a retraction is requested following an institutional misconduct investigation.

b. Publishers should reserve the right to retract in legal agreements with authors. ${ }^{23}$

c. Make sure that all journal websites provide clear instructions on how to submit an inquiry or concern about possible research misconduct or serious error. For instance, websites may not have updated contact information or email addresses.

d. Create a workflow template for starting a retraction inquiry and adopt a checklist of requisite information for a retraction notice. Encourage journal editors and institutions to use the template and checklist to coordinate and communicate about the retraction inquiry.

\section{Educate Stakeholders About Publication Correction Processes including Retraction and about Pre- and Post-Publication Stewardship of the Scholarly Record.}

For researchers and editors there is a tension between the need to correct the literature and the need to preserve their reputations. Fear of stigma or career impacts can make researchers reluctant to participate in retraction processes, even to correct honest mistakes or errors. Fear of litigation makes editors reluctant to initiate retraction inquiries (COPE Council, 2019b; Williams \& Wager, 2013). Awareness of retraction and the reasons for retracting research may vary by field; this contributes to a confusion about the severity and impacts of retraction.

\section{Individual Recommendations for Educating Stakeholders}

Researcher Education

1. Educate researchers on the value of amending the published literature, including through retraction. Promote correct understanding of retraction, peer review, and publication ethics as part of Responsible Conduct of Research education.

\footnotetext{
${ }^{23}$ COPE 2019: “Journals' instructions for authors should explain the journal's policies on publication ethics and describe the circumstances under which articles might be retracted. This information should be incorporated into author agreements and brought to the authors' attention."
} 
2. Emphasize the need to correct publications as part of responsible data management and work to destigmatize the act of retracting articles.

a. Develop an anthology of stories of honest retraction aimed at destigmatizing retraction amongst senior scientists. Retraction Watch's "doing the right thing" columns (Retraction Watch, n.d.-b) and The Winnower's stories of both honest retraction and misconduct are models of "letting it all hang out" (Baker et al., 2014; Linger \& Graham, 2014; Palestis et al., 2014; Stapel, 2014).

b. Move away from inflammatory language associated with misconduct. Naturalize a more neutral tone for retraction inquiry and reportage.

3. Develop retraction-focused best practice modules to be integrated into Responsible Conduct of Research education.

a. Develop pedagogical materials for use in broader ethics and professionalization training. This should include materials on different reasons for post-publication changes to publications.

b. Make materials available in open education platforms under licenses suitable for translation and reuse, to encourage multilingual availability.

Authors

1. Educate authors about their duties and responsibilities related to retraction and other post-publication amendments (e.g., editorial notes, corrections, and expressions of concern).

a. Authors have a responsibility to evaluate and assess the references in their bibliographies. In particular:

i. Before publication, authors are expected to cite and reference reliable papers in good standing. In cases where it is relevant to discuss retracted work, the article/text and bibliography should both clearly indicate the retracted status of the cited work.

ii. Before publication, authors are expected to provide correct and complete reference details. Authors are also expected to respond to questions about errors/omissions in bibliographies in manuscripts at the pre-press stage.

iii. After publication, authors of evidence synthesis products are expected to notify the journal if they notice that an article they cited has been retracted, because as COPE notes, "Articles that relied on subsequently retracted articles in reaching their own conclusions, such as systematic reviews or meta-analyses, may themselves need to be corrected or retracted" (COPE Council, 2019b). Amendments provided by previous authors (Habicher et al., 2011; Hovaguimian et al., 2019; Sheehan \& Beckett, 2016) can be used as examples.

b. Authors should take action to amend or retract their own research if compromised. If issues with an article come to light post-publication, an 
author should notify the publisher, their own institution, and all co-authors' institutions.

\section{Topic Editors}

a. Journal editors and others responsible for stewarding research on a topic should consider commissioning review articles to revisit the entirety of research on a topic, in the wake of a series of retractions or other post-publication amendments. Anesthesiology's 'what do we still know' series provides a model (Reinhart \& Takala, 2011; White et al., 2009).

\section{Editorial Education}

1. Build awareness of existing COPE resources and retraction best practices among editors, peer reviewers, and publishers.

a. Develop publication ethics training modules and cases for small publishers. Target museum and library-based publishers and other small scholarly publishers by developing modules to be integrated into the Library Publishing Curriculum (Educopia Institute, 2016).

2. Encourage efforts to destigmatize the decision to retract articles and educate editors about policies regarding previously retracted work.

a. Develop best practice educational modules that address the stigma associated with retraction.

b. Where necessary, develop educational materials to educate editors, peer reviewers, and publishers about honest inadvertent serious errors that may require retraction. Develop accompanying strategies for dissemination of this material.

3. Create a working group on preprint publishing ethics, building on the recommendations around withdrawal and removal of preprints (Beck et al., 2020) that were developed at the January 2020 ASAPbio, EMBL-EBI, and Ithaka S+R meeting.

4. Establish indexing guidelines and standards that support authors in identifying authoritative sources for checking citations, beyond Retraction Watch and PubMed. The ICMJE guidelines note that: "Authors are responsible for checking that none of the references cite retracted articles except in the context of referring to the retraction. For articles published in journals indexed in MEDLINE, the ICMJE considers PubMed the authoritative source for information about retractions." ${ }^{24}$ A working group could audit possible sources of retraction

\footnotetext{
${ }^{24}$ ICMJE Guidelines: "Authors are responsible for checking that none of the references cite retracted articles except in the context of referring to the retraction. For articles published in journals indexed in MEDLINE, the ICMJE considers PubMed the authoritative source for information about retractions. Authors can identify retracted articles in MEDLINE by searching PubMed for "Retracted publication [pt]",
} 
information in a variety of fields, and recommend authoritative sources of retraction information beyond medicine.

where the term "pt" in square brackets stands for publication type, or by going directly to the PubMed's list of retracted publications." (International Committee of Medical Journal Editors, 2019). 


\section{IMPLEMENTATION OF THE RECOMMENDATIONS}

Here, we offer implementation strategies in order for our recommendations above to be both adopted and institutionalized. Whereas the recommendations above refer to large crosssectoral areas for coordinated action, the implementation actions below describe more discrete actions (some suitable for independent action by a single stakeholder group) that help enact the recommendations and make them tangible over the long term.

\section{Implementation Actions for Recommendation \#1: Develop a systematic cross-industry} approach to ensure the public availability of consistent, standardized, interoperable, and timely information about retractions.

1. Encourage producers of major style guides to adopt styles for retracted literature that can be easily implemented in Citation Style Language (Citation Style Language, n.d.) using publisher metadata.

2. Support the Retraction Watch Database in developing an API and additional partnerships with data consumers.

3. Organize a working group and standing committee as a cooperative effort between NISO and COPE to develop a recommended practice or standard for how retractions/withdrawal should be displayed on websites. Model standards development on the Recommended Practices for the Presentation and Identification of E-Journals (PIE-J) (NISO PIE-J Working Group, 2013).

4. Translate the COPE and ICMJE guidelines into frameworks and checklists that can be adopted and scoped to the needs of multiple stakeholders groups. Make such tools available in multiple languages.

A starting point could be the following framework amending research developed for this project (Schneider et al., 2020) which interprets the COPE and ICMJE guidelines.

i. All search results for the title, author(s), or subject(s) of the retracted article should also return the retraction notice. ${ }^{25}$

ii. For retracted articles on publisher and full-text sites: ${ }^{26} 27$

${ }^{25}$ COPE 2019 on search: "The retraction should appear on all online searches for the retracted publication." (COPE Council, 2019b)

${ }^{26}$ COPE 2019 on visibility of retracted articles: "Retracted articles should be unmistakably identified as such in all online sources (eg, on the journal website, on the original article, and any bibliographic databases). Journals are responsible for ensuring that retractions are labelled in such a way that they are identified by bibliographic databases and should also include a link to the retracted article. The retraction should appear on all online searches for the retracted publication." And: "Retraction notices should be published in all versions of the journal (ie, print and/or online)." (COPE Council, 2019b)

27 ICMJE on retractions "Expressions of concern and retractions should not simply be a letter to the editor. Rather, they should be prominently labelled, appear on an electronic or numbered print page that 
1. Each article landing page, full-text HTML article, and full-text PDF article should have a phrase indicating the retraction status (such as 'retracted,' 'withdrawn,' etc.) or a watermark indicating the retraction status.

2. Each landing page, full-text HTML article, and full-text PDF article should have a computer-actionable link to the retraction notice.

b. For retraction notices in publisher and full-text sites: ${ }^{28}$

i. The retraction notice should appear in the Table of Contents for the issue in which it appears, with a designated page number.

ii. The heading of the retraction notice should include 'retraction' or 'retraction notice' and the title of the retracted article.

iii. The textual content of the retraction notice should state authorship, reason for retraction, and formally cite the retracted article. It should be made available as an open access publication (regardless of whether the retracted article was published open access).

iv. Each landing page, full-text HTML notice, and full-text PDF notice should have a computer-actionable link to the retracted article.

c. For database records for retracted articles:

i. A phrase indicating the retraction status, such as 'retraction,' 'retracted,' or 'withdrawn' should appear in the article record.

ii. The article record should have a computer-actionable link to the retraction notice. This could link to the database's record for the retraction notice, or to the full-text retraction notice.

is included in an electronic or a print Table of Contents to ensure proper indexing, and include in their heading the title of the original article. Online, the retraction and original article should be linked in both directions and the retracted article should be clearly labelled as retracted in all its forms (Abstract, full text, PDF). Ideally, the authors of the retraction should be the same as those of the article, but if they are unwilling or unable the editor may under certain circumstances accept retractions by other responsible persons, or the editor may be the sole author of the retraction or expression of concern. The text of the retraction should explain why the article is being retracted and include a complete citation reference to that article. Retracted articles should remain in the public domain and be clearly labelled as retracted. (International Committee of Medical Journal Editors, 2019)

${ }^{28}$ COPE 2019 on retraction notices:

"Notices of retraction should:

- Be linked to the retracted article wherever possible (ie, in all online versions)

- Clearly identify the retracted article (eg, by including the title and authors in the retraction heading or citing the retracted article)

- Be clearly identified as a retraction (ie, distinct from other types of correction or comment)

- Be published promptly to minimise harmful effects

- Be freely available to all readers (ie, not behind access barriers or available only to subscribers)

- State who is retracting the article

- State the reason(s) for retraction

- Be objective, factual and avoid inflammatory language." (COPE Council, 2019b) 
iii. The article record has sufficient bibliographic information to retrieve the retraction notice.

d. For database records for retraction notices:

i. A phrase such as 'retraction' or 'retraction notice' should appear in the text of the notice record.

ii. The notice record should have a computer-actionable link to the retracted article. This could link to the database's record for the retracted article, or to the full-text retracted article.

iii. The notice record has sufficient bibliographic information to retrieve the retracted article.

\section{Implementation Actions for Recommendation \#2: Recommend a taxonomy of retraction categories/classifications and corresponding retraction metadata that can be adopted by all stakeholders.}

1. One outcome of the stakeholder workshop is the formation of a COPE working group to develop a taxonomy of retraction categories. Based on the developments of this working group, we suggest that COPE partner with other other organizations, such as NISO or STM, in consultation with Crossref and others, to steward the development of this taxonomy as a more commonly adopted standard. COPE can provide ethics-related guidance and resources to publishers and editors, but if this taxonomy is to be broadly adopted, it must include wide-ranging input from a greater diversity of stakeholders in the scholarly publishing ecosystem. A standards organization would be well positioned to facilitate that process.

\section{Implementation Actions for Recommendation \#3: Develop best practices for coordinating the retraction process to enable timely, fair, and unbiased outcomes.}

1. Form a cross-association working group to examine the coordination of retraction processes and to identify where retraction can be anchored in institutional policies. Membership should be drawn from COPE, the Association of Research Integrity Officers (ARIO), STM, and NISO. This is particularly timely following the CLUE report (Wager et al., 2021) and as COPE is in the process of expanding its membership to include institutions.

Example action areas for the working group or its subgroups could include:

a. Recommending workflows for retractions and amendments related to figures and images.

b. Clarifying guidelines and workflows for expediting retractions and other amendments if the authors agree with or request retraction, or if a retraction is requested following an institutional misconduct investigation. 
2. Support the development of research data ethics guidelines through the FORCE11 Working Group on Research Data Publishing Ethics (FORCE11, 2020), in collaboration with COPE. Work towards the adoption of the guidelines when they become available.

3. Support COPE initiatives to develop workflows that incorporate standardized retraction forms.

a. Refine the European Association of Science Editors (EASE) standardized retraction form (The European Association of Science Editors, 2021). EASE is currently asking questions to facilitate implementation, such as Is a simplified version needed? Is this form intended for journals of learned societies and/or large publishing houses? How can we better promote the form?

b. Develop workflows to incorporate this form.

c. Translate the form and workflow template/guidance into many languages to facilitate broad dissemination to a global user group.

d. Incorporate workflow and template guidance into the COPE flowchart library which is already commonly used and referenced by editors and publishers.

\section{Implementation Actions for Recommendation \#4: Educate stakeholders about publication correction processes including retraction and about pre- and post- publication stewardship of the scholarly record.}

1. Existing materials developed by COPE may not be widely distributed beyond COPE membership. Develop a working group to evaluate and build upon COPE resources for editors and create supplemental case studies to illustrate best practices regarding retraction. Develop and disseminate educational materials to editors and journals that focus on education for editors. These resources can be utilized in the professional development of editors, as well as in formal training programs in publishing.

2. Form a cross-industry working group to develop and disseminate publication ethics and retraction-related case studies aimed at researchers to be used in RCR education. This work could be led by a group such as the National Center for Professional and Research Ethics (NCPRE) and gather members from associations such as COPE and ARIO and national funders such as (in the United States) the National Institutes of Health and the National Science Foundation.

3. Form a cross-industry working group to develop guidelines for amendments to research products (e.g., updates, corrections, retractions, withdrawals, etc.) including data and datasets, software and code, and methods protocols. Explore whether a new forum is needed or whether COPE membership is appropriate for stewards of research products that do not lead to a Version of Record. Standards groups such as NISO could foster discussions to build on FORCE11's Research Data Publishing Ethics Group and their collaboration with COPE (COPE Council, 2021; FORCE11, 2020). 
4. Develop and promote guidelines for amending evidence synthesis products such as systematic reviews and meta-analyses when the research they synthesize has been retracted. Authors have noted the need for such guidelines (Faggion, 2019). 


\section{RESEARCH AGENDA}

Building off of our implementation agenda, we outline below a research agenda that highlights areas for future research, with some recommendations as to scope and approach. We hope that these suggestions for further research will also be taken up by other researchers. Further study in this area will aid the implementation actions we recommend above. This map of further research should also help to consolidate an explicit, community-wide research program on retraction, retracted research, and problems associated with its continued citation and use.

\section{Research Actions for Recommendation \#1: Develop a systematic cross-industry approach to ensure the public availability of consistent, standardized, interoperable, and timely information about retractions.}

1. Systematically assess the currently available information about retraction.

a. Compare retraction indexing in multidisciplinary databases such as Crossref, Retraction Watch, Scopus, and Web of Science.

i. Expand research undertaken for this project, which was limited to the subset of items whose titles start with "RETRACTED:" or similar (Proescholdt \& Schneider, 2020). The RISRS team investigated retraction indexing in Scopus and Web of Science (as well as the biomedical database PubMed), reported data quality problems to publishers, and continues to follow up about likely errors.

b. Investigate how frequently retractions are signalled at popular multidisciplinary search engines such as Google Scholar and Semantic Scholar.

c. Identify which subject-specific and multidisciplinary databases provide authoritative information about retracted papers in a given field, for a variety of fields beyond the biomedical sciences.

2. Do differences in citation style guidelines, and reference managers/citation software, impact whether and how people cite retracted papers? Examine citation style guidelines and reference managers in order to determine whether and how these differences impact the citation of retracted papers.

3. Compare how in-field and out-of-field researchers receive information about retractions. Media attention to two large-scale misconduct-related retractions may have contributed to a drop in post-retraction citations (Mott et al., 2019). The percentage of out-of-field citations seemed to increase, post-retraction citation, in one misconduct case (Bornemann-Cimenti et al., 2016).

a. What role do media attention and simultaneous retraction of multiple papers play in researchers' citation behavior?

b. Does the percentage of out-of-field citations increase after retraction?

4. Examine variation in retraction-related publishing policy and process through a series of synthetic case studies. For COPE guidelines, for instance, this could help distinguish 
adherence (e.g., non-COPE members) versus compliance (e.g., COPE members not following the guidelines).

5. Systematically review and assess retraction notice quality. Examine retraction notice quality in a granular time scale, sampling from multiple databases, and evaluate retraction notice quality beyond the minimum requirements of the COPE guidelines (COPE Council, 2019b).

6. Develop a series of evaluative, best-practice-oriented case studies that examine how infrastructures impact information quality. In particular, examine how the design, development, implementation and sustainability of information systems such as publication websites, databases, and platforms has changed over time, and how this impacts metadata flows.

a. As a part of this research, develop case studies focused on the exchange of retraction information amongst publishers, libraries, and database providers. Although publishers may regularly send out updates, sometimes several times a day, recipients find it time-consuming to digest and quality check bulk downloads. What best practices would make this process more manageable (e.g., particular procedures and workflows, widely distributed utility diffs and data comparison tools)?

\section{Research Actions for Recommendation \#2: Recommend a taxonomy of retraction} categories/classifications and corresponding retraction metadata that can be adopted by all stakeholders.

1. Evaluate the utility and impact of the taxonomy being developed by COPE and partners, once developed. In particular, identify any barriers to adoption.

a. How much of the publishing and information services organizations adopt the taxonomy?

b. As a research program, track and monitor the consistency of retraction metadata across platforms.

i. Evaluate whether all retracted items are identified through relevant retraction metadata.

\section{Research Actions for Recommendation \#3: Develop best practices for coordinating the retraction process to enable timely, fair, and unbiased outcomes.}

1. Examine potential institutional pathways for universities to implement guidelines and protocols for evaluating a researcher's record of retraction and post-publication amendments. Consider how to integrate best practice guidelines into research integrity policies and procedures so that retractions are not dealt with completely on an ad hoc basis. Encourage institutions to develop authorship guidance which emphasizes the 
need to correct publications (including retraction, if necessary) as an expectation of responsible research.

a. Examine institutional pathways to naturalize researchers engaging in retraction to clean up the literature, without penalty for honest error.

b. Examine institutional pathways to strengthen negative incentives for misconduct that result in retraction.

c. Research the role of funding agencies in guiding recipients' institutional policies. Develop models of guidance and best practices.

2. Research the impact of retractions on individual and institutional reputations. Develop case studies that support the development of best practice guidelines, especially for retractions involving students, contributing scholars, early career researchers, and candidates for promotion and tenure reviews.

3. Develop comparative case studies to understand the legal environment for retraction, which sits in a complex nexus amongst publishers, researchers, and institutions.

Develop governance and policy guidelines to facilitate, where possible greater, coordination between journals, institutions, and other stakeholders, such as funders and government agencies.

4. Investigate how to create reporting conduits that protect privacy but disincentivize the threat of retraction due to academic infighting, personal vendettas and other forms of 'data bullying.'

5. Develop case studies of university researchers navigating the retraction process, particularly for cases of honest error, building off of Hosseini (2018). What are the social and institutional impediments to this process?

a. Consider that for researchers based at universities, for example, retracting a publication may involve a coordinated process amongst publishers and editors, multiple authors, and institutional officers, and in some cases, lawyers. The legal, reputational, and financial negotiations and outcomes of these processes are complex, and this process potentially slows down retraction and often makes it expensive.

6. Investigate the costs associated with adopting innovations in the scholarly publishing ecosystem. Examine the barriers to stakeholder adoption and guidance in the use of best practices and emerging technologies.

a. Research the pros and cons of providing provenance as to how retractions were detected, for instance in order to credit software used to detect problems and whistleblowers who prefer publicity to anonymity.

b. Research the introduction of workflows and technologies to support peer review for common problems that can be detected by trained personnel and/or by automatic analyses. 
c. Examine the financial impacts of retraction best practices on small publishers. Are there ways to offset or mitigate the costs associated with retraction?

d. Gather and evaluate evidence about the impact of different approaches to the retraction process. For instance, which is a more effective strategy for addressing the issue of timeliness: retracting as an immediate, first step, and then publicizing reasons later; or, providing reasons for retraction at the same time as the retraction?

\section{Research Actions for Recommendation \#4: Educate stakeholders about publication correction processes including retraction and about pre- and post-publication stewardship of the scholarly record.}

1. Research resources and standards in different global contexts pertaining to Responsible Conduct of Research, publication ethics, and retraction. This could improve understanding of the varying contexts in which researchers work and differences in availability of integrity-focused resources and training. Ultimately this could increase cultural sensitivity by multiple stakeholders around integrity issues and inform and prioritize efforts to develop resources addressing region-specific knowledge gaps.

2. Survey and evaluate existing models for educating researchers about the publication process. As an outcome of this research, develop educational materials that address retraction and best practices in educating researchers about navigating the scholarly communications ecosystem.

3. After publication, authors are strongly encouraged to monitor the papers, cited and noncited, which form a basis for their work. Authors are encouraged to notify journals when a work they cited becomes retracted, or has a significant post-publication amendment. This includes papers that were not retracted at the time the bibliography was developed, but subsequently becomes retracted, because regardless of when a retraction occurs, it may impact the citing work (Fu \& Schneider, 2020; Marcus, 2020).

4. Examine the role of stigma in the perception of retracted research. Is it possible to distinguish reasonable skepticism from undue bias towards authors of retracted work? Analyze the institutionalized norms supporting the destigmatization of article retraction from the authors' and editors' standpoints. Consider whether transparent policies are needed for consideration of previously retracted work.

5. Research and develop best practice guidelines for managing the impact of retractions on individual and institutional reputations.

a. Guidelines should specifically address career development for students, contributing scholars and early career researchers. Guidelines should address issues of advancement and promotion at all stages of academic careers, including the tenure and promotion review process. 
b. Guidelines should illustrate best practices for working with researchers who selfretract for reasons of error.

c. Guidelines should address whistleblowing activity within university and research institution settings and with awareness of structural inequality, power differences, contingency, and risks of retribution. Careful attention should be placed on differential power dynamics involved in the student-advisor relationship and between Primary Investigators and sponsored staff in non-permanent, grantfunded roles such as postdoctoral researchers, research assistants, and technicians.

6. Establish a research program to examine the role of peer review in retracted research. Synthesize the literature on recommendations for reforming peer review and investigate by discipline.

7. Investigate how disciplinary and professional societies can incentivize authors to steward their publications, making post-publication changes when fundamental errors are identified.

8. Editorial guidelines and resources devoted to publication ethics and integrity may play a role in publisher-level and/or journal-level differences. Research why some editors and publishers do not use COPE resources.

a. Compare COPE member journals to non-COPE member journals and investigate whether there is an information gap that could be addressed by some manner of communication or outreach between these two journal subsets.

b. Survey journals and researchers regarding their use of and adherence to COPE and ICMJE standards, and design targeted case studies to identify the barriers to full adoption of the COPE and ICMJE guidelines.

c. Develop focus groups to determine whether stakeholders find these guidelines to be useful in navigating the retraction process.

9. Investigate editors' resources and capacities for engaging in retraction processes.

Examine the role of publishers and editorial boards in developing appropriate procedures, policies, and capacities to support editors in retraction processes.

10. Determine the rate at which retractions are inappropriate according to the guidelines; this is a topic that has received only limited study (Huh et al., 2016).

11. Investigate the barriers for authors in (1) noticing that a publication they cited has been retracted, and (2) alerting the journal, with an indication of whether or not the citing article needs a post-publication amendment. Consider what technologies or other resources authors and editors would need for effective stewardship and updating of the citing literature. Evaluate the effectiveness and ease of use of possible technologies and workflows that address some of these barriers. 


\section{Research Actions Related to RISRS Process Outcomes.}

Track, document, and evaluate the standards-setting processes initiated to address the continued citation of retracted research. Standards-setting processes are critically understudied, particularly related to their institutionalization in contexts for which there is little agreement or no strong responsible agent.

1. Crucial general questions to consider include:

a. How do the standards meet the various usability expectations of the stakeholder communities?

b. How will the standards be implemented in online publication systems?

i. Are there organizational barriers to implementation?

ii. Are there technological barriers to implementation?

c. How are the standards disseminated?

2. Track, document and evaluate the processes around the introduction of a taxonomy of retraction categories/classifications.

a. How does the recommended taxonomy meet the various usability expectations of the stakeholder communities?

b. How will the taxonomy be implemented in online publication systems?

i. Are there organizational barriers to implementation?

ii. Are there technological barriers to implementation?

c. How will the taxonomy be disseminated?

i. What are the impediments to dissemination and stakeholder buy-in?

3. Track, document, and evaluate the processes and progress of the proposed NISO work item on Communication of Retractions, Removals, and Expressions of Concern CORREC. This NISO-led standards setting process will establish recommended practices that address "the dissemination of retraction information (metadata \& display) to support a consistent, timely transmission of that information to the reader (machine or human), directly or through citing publications, addressing requirements both of the retracted publication and of the retraction notice or expression of concern."

a. Develop a series of case studies that explore adoption of the proposed best practices, to understand alignment with existing practices, and barriers to adoption.

i. Examine adoption throughout the scholarly communications ecosystem, profiling publishers, abstracting and indexing providers, link resolvers, researchers, journalists, repositories, etc.

ii. Examine adoption and development by influential actors such as COPE, the ICMJE and the Council of Science Editors.

b. Track and evaluate:

i. The recommended additions to existing metadata deliverables, as examples.

ii. The proposed channels by which retraction information is distributed. 
iii. The suggested best practices around populating metadata in retraction notices, as well as in retracted publications, and expressions of concern.

iv. The identification of who is responsible for creation of metadata and what are the subsequent responsibilities of consumers of those metadata, e.g., display and indexing of retraction-related metadata.

v. The best practices around the communication of the item's status, including display labels, information available to support accessibility and machine processes, etc.

vi. The recommendations regarding the visibility of the retracted digital object and consistency in signaling that status. Are there different solutions needed for different expressions of objects, e.g., will the requirements for a PDF version differ from those for an HTML, EPUB, or XML version?

vii. The introduction of an illustrative workflow process from issuing a retraction notice, through display \& discovery of the retracted item; implementation will be modeled and scalability will be considered.

\section{Research Actions Related to RISRS Citation Research.}

1. Develop a framework and guidelines to determine the downstream impact of retracted research.

a. Investigate items retracted or corrected due to citation of a retracted paper (e.g., the 127 items in the Cited Retracted Work retraction reason category at the Retraction Watch Database).

b. Develop a theoretical framework to determine when a publication is logically dependent on a retracted work.

c. Develop guidelines to help publishers determine when publications citing retracted research require post-publication amendments. This should draw on the theoretical framework and existing guidelines (COPE Council, 2019b; Cumpston \& Chandler, 2021) in order to respond to past research about the impact of retracted research, and commentary on the lack of agreement on when post-publication amendment is necessary (Marcus, 2021).

d. Develop guidelines to help policymakers gauge the impact to policy decisions using retracted research as evidence.

2. Research feasibility and implementation of post-publication amendments of publications depending on retracted research.

a. Identify user needs and feasibility requirements in making post-publication amendments of publications that depend on retracted research. Users might include authors, publishers, repository managers, etc.

b. Develop automated solutions and strategies to facilitate post-publication amendments of publications that depend on retracted research.

c. Test the practicality. For instance, automatically identify dependencies in collaboration with evidence synthesis organizations (e.g., the citations used as evidence in systematic reviews and meta-analyses). 
3. Research the impact that retraction has on non-publication objects that are related to or created from retracted research.

a. Scientific Knowledge Integration Products

i. Develop case studies to investigate how scientific knowledge integration products, which are often constructed using data or knowledge extracted from scientific publications, currently handle retracted research. Case studies will need to consider various types such as scientific knowledge graphs, integrative databases, and integrative data analytic platforms.

ii. Identify requirements for dynamically maintaining scientific knowledge integration products, based on the case studies.

b. Data, Code, and other Related Research Products

i. Develop case studies to investigate how retraction of a publication has impacted related research objects (e.g., data, code). Case studies will need to consider multiple reasons for retraction and multiple types of related research objects.

ii. Propose guidelines for handling related research objects in response to retraction of a related work. In addition to retraction, significant updates may need to be considered (e.g., flawed computer code that is updated rather than retracted).

4. Research the impact that retraction of non-publication objects has on related publications.

a. Develop case studies to investigate how retracted datasets affect publications depending on them. What are the legal, social, and ethical ramifications? Research solutions to manage the aftermath of dataset retractions.

b. Develop case studies to investigate how flawed computer code affects publications using them. Where are such computer codes published and shared? Are there standard procedures to retract flawed computer code? Research solutions to manage publications using computer code that is later discovered to be flawed.

\section{CONCLUSIONS}

The goal of the RISRS project has been to clarify the scope and dimensions of the problem of continued citation of retracted research, and to develop a research and implementation agenda for reducing its spread. In this conclusion, we briefly cover some aspects of our implementation strategy, and describe ways for you to contribute to the collaborative development of this agenda.

Our general approach to this problem has been to co-develop a set of actionable recommendations with a diverse group of stakeholders associated with scholarly publishing. To do this, we initiated a scoping literature review and created multiple opportunities for sustained stakeholder consultation. Both enabled a process of problem definition and refinement where 
we actively worked with stakeholders to identify viable areas on which to focus our attention, and to anchor recommendations in the terrain of the scholarly communications ecosystem.

\section{Implementation Strategy}

We have outlined possible cross-sectoral strategies for introducing change throughout the scholarly publishing ecosystem, and hope that this encourages collaboration amongst stakeholders from different industries to collaborate to address the continued citation and use of retracted research.

Our aim is for stakeholders to take up our recommendations as elements of an implementation agenda that targets reducing the continued citation and use of retracted research. We have prioritized recommendations that are immediately and practically actionable, and which will support robust collaboration between stakeholders utilizing existing practices, policies, standards and technologies.

We suggest stakeholders prioritize recommendations \#1 Develop a systematic crossindustry approach to ensure the public availability of consistent, standardized, interoperable, and timely information about retractions, and \#2 Develop taxonomy of retraction categories/classification and corresponding retraction metadata that can be adopted by all stakeholders. First, the remaining recommendations will have more traction once some agreement around recommendations \#1 and \#2 has developed. Second, some progress on these recommendations has already been made.

NISO has convened meetings to consider developing a recommended practice or a standard for how retractions/withdrawals should be handled by publishers, as a follow up from a RISRS cross-industry panel at NISO Plus 2021. We expect NISO standardization work, if taken up, to follow up on Recommendations \#1 and/or \#2.

There was broad stakeholder agreement during the workshop that a taxonomy and metadata (Recommendation \#2) was a necessary first step, and a COPE working group has already produced an initial draft of the taxonomy, described by Deborah Poff at the Society for Scholarly Publishing 2021 conference. The next step will be for multiple stakeholders to assess the taxonomy, document what works, iterate as necessary, and encourage adoption. NISO or another standards body may be an appropriate long-term steward for this taxonomy.

\section{A Call to Implement and Collaborate}

Addressing the inadvertent, continued citation of retracted science will require iterative work from many parts of the scholarly publishing ecosystem. We encourage you to disseminate these recommendations and to envision how you, in your role, and in collaborative partnerships, can make a difference. Feel free to involve RISRS participants and the authors of this document in implementation actions to enact this vision! We welcome suggestions for ongoing research and collaboration, via the project website https://infoqualitylab.org/projects/risrs2020/ or by email to jodi@illinois.edu. 


\section{LIST OF APPENDICES}

Appendix A: RISRS Project Outcomes to Date

Appendix B: Problems and Opportunities Dataset

Appendix C: Literature Scoping Review Methods and Intermediate Results

Appendix D: Stakeholder Consultation Process

Appendix E: Existing Citation Standards for Retracted Publications

\section{CRediT CONTRIBUTOR STATEMENT FOR THE RECOMMENDATIONS}

Yoss Arianlou: Investigation, Writing - original draft, Writing - review \& editing; Yee Yan

'Vivien' Yip: Investigation; Halle Burns: Investigation; Mary Terese Campbell: Project administration; Yuanxi Fu: Investigation, Writing - original draft, Writing - review \& editing;

Katherine Howell: Investigation, Writing - review \& editing; Tzu-Kun (Esther) Hsiao: Investigation; Randi Proescholdt: Conceptualization, Data curation, Formal analysis, Investigation, Methodology, Project administration, Writing - original draft, Writing - review \& editing; Jodi Schneider: Conceptualization, Data curation, Formal analysis, Funding acquisition, Investigation, Methodology, Project administration, Resources, Supervision, Writing - original draft, Writing - review \& editing; Nathan D. Woods: Conceptualization, Data curation, Formal analysis, Investigation, Methodology, Project administration, Writing - original draft, Writing - review \& editing.

\section{ACKNOWLEDGEMENTS}

The project "Reducing the Inadvertent Spread of Retracted Science: Shaping a Research and Implementation Agenda" was supported by Alfred P. Sloan Foundation G-2020-12623. This work was made possible by the participation of our interviewees and workshop attendees. We gratefully acknowledge their contributions and feedback. Thank you to the wider community who joined in providing post-workshop feedback on this document, including Alison Avenell, Jennifer Byrne, Renee Hoch, Hervé Maisonneuve, and Iraxte Puebla.

The RISRS Team: Halle Burns, Jodi Schneider, Katherine Howell, MT Campbell, Nathan D. Woods, Randi Proescholdt, Tzu-Kun (Esther) Hsiao, Yee Yan 'Vivien' Yip, Yuanxi Fu, Yoss Arianlou

\section{BIBLIOGRAPHY}

Abbott, A. (2019). The science institutions hiring integrity inspectors to vet their papers. Nature, 575(7783), 430-433. https://doi.org/10.1038/d41586-019-03529-w

Abritis, A. (2016, January 22). You've been dupe'd: Meet authors who like their work so much, they publish it twice. Retraction Watch. https://retractionwatch.com/2016/01/22/youvebeen-duped-again/

Abritis, A., Marcus, A., \& Oransky, I. (2021). An "alarming" and "exceptionally high" rate of COVID-19 retractions? Accountability in Research, 28(1), 58-59.

https://doi.org/10.1080/08989621.2020.1793675 
Al, U., \& Soydal, I. (2017). Publication lag and early view effects in information science journals. Aslib Journal of Information Management, 69(2), 118-130. https://doi.org/10.1108/AJIM12-2016-0200

Alberts, B., Cicerone, R. J., Fienberg, S. E., Kamb, A., McNutt, M., Nerem, R. M., Schekman, R., Shiffrin, R., Stodden, V., Suresh, S., Zuber, M. T., Pope, B. K., \& Jamieson, K. H. (2015). Self-correction in science at work. Science, 348(6242), 1420-1422. https://doi.org/10.1126/science.aab3847

Al-Ghareeb, A., Hillel, S., McKenna, L., Cleary, M., Visentin, D., Jones, M., Bressington, D., \& Gray, R. (2018). Retraction of publications in nursing and midwifery research: A systematic review. International Journal of Nursing Studies, 81, 8-13. https://doi.org/10.1016/j.ijnurstu.2018.01.013

American Psychological Association. (2019). Publication Manual of the American Psychological Association (7th Edition). American Psychological Association.

Athar, A. (2011). Sentiment analysis of citations using sentence structure-based features. Proceedings of the ACL-HLT 2011 Student Session, 81-87.

Atlas, M. C. (2004). Retraction policies of high-impact biomedical journals. Journal of the Medical Library Association: JMLA, 92(2), 242-250. https://www.ncbi.nlm.nih.gov/pmc/articles/PMC385306/

Atypon Systems. (2013, March 14). EXtyles implements CrossRef retraction warnings. Inera. https://www.inera.com/blog/extyles-implements-crossref-retraction-warnings/

Azoulay, P., Bonatti, A., \& Krieger, J. L. (2017). The career effects of scandal: Evidence from scientific retractions. Research Policy.

Azoulay, P., Furman, J. L., Krieger, \& Murray, F. (2015). Retractions. Review of Economics and Statistics, 97(5), 1118-1136. https://doi.org/10.1162/REST_a_00469

Baker, R., Van den Bussche, R., Wright, A., Wiggins Johnson, L., Hamilton, M., Reat, E., Smith, M., Lomakin, M., \& Chesser, R. (2014). A retrospect on achievements following an onerous retraction. The Winnower. https://doi.org/10.15200/winn.140076.65836

Bakker, C., \& Riegelman, A. (2018). Retracted publications in mental health literature: Discovery across bibliographic platforms. Journal of Librarianship and Scholarly Communication, 6(1), eP2199. https://doi.org/10.7710/2162-3309.2199

Balhara, Y. P. S., \& Mishra, A. (2014). Compliance of retraction notices for retracted articles on mental disorders with COPE guidelines on retraction. Current Science, 107(5), 757-760.

Barbour, V., Bloom, T., Lin, J., \& Moylan, E. (2017). Amending published articles: Time to rethink retractions and corrections? [version 1; peer review: 2 approved with reservations]. F1000Research, 6, 1960. https://doi.org/10.12688/f1000research.13060.1

Bar-Ilan, J., \& Halevi, G. (2017). Post retraction citations in context: A case study. Scientometrics, 113(1), 547-565. https://doi.org/10.1007/s11192-017-2242-0

Bar-llan, J., \& Halevi, G. (2018). Temporal characteristics of retracted articles. Scientometrics, 116(3), 1771-1783. https://doi.org/10.1007/s11192-018-2802-y

Beck, J., Ferguson, C. A., Funk, K., Hanson, B., Harrison, M., Ide-Smith, M., Lammey, R., Levchenko, M., Mendonça, A., Parkin, M., Penfold, N., Pfeiffer, N., Polka, J., Puebla, I., Rieger, O. Y., Rittman, M., Sever, R., \& Swaminathan, S. (2020). Building trust in preprints: Recommendations for servers and other stakeholders. OSF Preprints. https://doi.org/10.31219/osf.io/8dn4w

Bik, E. M., Casadevall A., \& Fang F.C. (2016). The prevalence of inappropriate image duplication in biomedical research publications. MBio, 7(3). https://doi.org/10.1128/mBio.00809-16

Bik, E. M., Fang, F. C., Kullas, A. L., Davis, R. J., \& Casadevall, A. (2018). Analysis and correction of inappropriate image duplication: The molecular and cellular biology experience. Molecular and Cellular Biology, 38(20), e00309-18.

https://doi.org/10.1128/MCB.00309-18 
Bilbrey, E., O'Dell, N., \& Creamer, J. (2014). A novel rubric for rating the quality of retraction notices. Publications, 2(1), 14-26. https://doi.org/10.3390/publications2010014

Bishop, D. V. M. (2018). Fallibility in science: Responding to errors in the work of oneself and others. Advances in Methods and Practices in Psychological Science, 1(3), 432-438. https://doi.org/10.1177/2515245918776632

Bolboacă, S. D., Buhai, D.-V., Aluaș, M., \& Bulboacă, A. E. (2019). Post retraction citations among manuscripts reporting a radiology-imaging diagnostic method. PLOS ONE, 14(6), e0217918. https://doi.org/10.1371/journal.pone.0217918

Bolland, M. J., Grey, A., \& Avenell, A. (2021). Citation of retracted publications: A challenging problem [Online First]. Accountability in Research. https://doi.org/10.1080/08989621.2021.1886933

Bornemann-Cimenti, H., Szilagyi, I. S., \& Sandner-Kiesling, A. (2016). Perpetuation of retracted publications using the example of the Scott S. Reuben case: Incidences, reasons and possible improvements. Science and Engineering Ethics, 22(4), 1063-1072. https://doi.org/10.1007/s11948-015-9680-y

Boxheimer, E. W., \& Pulverer, B. (2019). Self-correction prevents withdrawal syndrome. The EMBO Journal, 38(18), e70001. https://doi.org/10.15252/embj.201970001

Brainard, J., \& You, J. (2018). What a massive database of retracted papers reveals about science publishing's 'death penalty.' Science. https://doi.org/10.1126/science.aav8384

Bramstedt, K. A. (2020). The carnage of substandard research during the COVID-19 pandemic: A call for quality. Journal of Medical Ethics, 46, 803-807. https://doi.org/10.1136/medethics-2020-106494

Braun, V., \& Clarke, V. (2019). Reflecting on reflexive thematic analysis. Qualitative Research in Sport, Exercise and Health, 11(4), 589-597. https://doi.org/10.1080/2159676X.2019.1628806

Bucci, E. M. (2018). Automatic detection of image manipulations in the biomedical literature. Cell Death \& Disease, 9(3), 400. https://doi.org/10.1038/s41419-018-0430-3

Budd, J. M., Coble, Z., \& Abritis, A. (2016). An investigation of retracted articles in the biomedical literature. Proceedings of the Association for Information Science and Technology, 53(1), 1-9. https://doi.org/10.1002/pra2.2016.14505301055

Budd, J. M., Coble, Z. C., \& Anderson, K. M. (2011). Retracted publications in biomedicine: Cause for concern. Association of College and Research Libraries Conference, 390395.

https://www.ala.org/acrl/sites/ala.org.acrl/files/content/conferences/confsand preconfs/nat ional/2011/papers/retracted_publicatio.pdf

Budd, J. M., Sievert, M., \& Schultz, T. R. (1998). Phenomena of retraction: Reasons for retraction and citations to the publications. JAMA, 280(3), 296. https://doi.org/10.1001/jama.280.3.296

Budd, J. M., Sievert, M., Schultz, T., \& Scoville, C. (1999). Effects of article retraction on citation and practice in medicine. Bulletin of the Medical Library Association, 87(4), 437-443. https://www.ncbi.nlm.nih.gov/pmc/articles/PMC226618/

Cagney, H., Horton, R., James, A., Kleinert, S., Nyakoojo, Z., Pryce, L., Grainger, E., Stanley, D., \& Wang, H. (2016). Retraction and republication-A new tool for correcting the scientific record? European Science Editing, 42(1), 3-7. https://www.ease.org.uk/wpcontent/uploads/orig_article_1.pdf

Callaway, E. (2015). Faked peer reviews prompt 64 retractions. Nature News. https://doi.org/10.1038/nature.2015.18202

Carlisle, J. B. (2012). The analysis of 168 randomised controlled trials to test data integrity: Analysis of 168 randomised controlled trials to test data integrity. Anaesthesia, 67(5), 521-537. https://doi.org/10.1111/j.1365-2044.2012.07128.x 
Carlisle, J. B. (2017). Data fabrication and other reasons for non-random sampling in 5087 randomised, controlled trials in anaesthetic and general medical journals. Anaesthesia, 72(8), 944-952. https://doi.org/10.1111/anae.13938

Casadevall, A., Steen, R. G., \& Fang, F. C. (2014). Sources of error in the retracted scientific literature. The FASEB Journal, 28(9), 3847-3855. https://doi.org/10.1096/fj.14-256735

Chakraborty, J., Pradhan, D., Dutta, H. S., Nandi, S., \& Chakraborty, T. (2018). On Good and Bad Intentions behind Anomalous Citation Patterns among Journals in Computer Sciences. https://arxiv.org/abs/1807.10804v1

Chawla, D. S. (2016, November 21). Oops-Springer journals retract three articles published by accident. Retraction Watch. https://retractionwatch.com/2016/11/21/oops-springerjournals-retract-three-articles-published-by-accident/

Chawla, D. S. (2019, November 22). Don't let researchers recommend who reviews their work. Nature Index. https://www.natureindex.com/news-blog/dont-let-researchers-choose-whopeer-reviews-their-work

Chen, C., Hu, Z., Milbank, J., \& Schultz, T. (2013). A visual analytic study of retracted articles in scientific literature. Journal of the American Society for Information Science and Technology, 64(2), 234-253. https://doi.org/10.1002/asi.22755

Christiansen, S., Iverson, C., \& Flanagin, A. (2020). AMA Manual of Style: A Guide for Authors and Editors (11th ed). Oxford University Press.

Citation Style Language. (n.d.). Citation Style Language. Retrieved March 12, 2021, from https://citationstyles.org/

Conceição, S. C. O., Samuel, A., \& Biniecki, S. M. Y. (2017). Using concept mapping as a tool for conducting research: An analysis of three approaches. Cogent Social Sciences, 3(1), 1404753. https://doi.org/10.1080/23311886.2017.1404753

COPE Council. (n.d.). COPE Members. COPE: Committee on Publication Ethics. Retrieved March 5, 2021, from https://publicationethics.org/members

COPE Council. (1999). Author dispute concerning ownership of data (No. 99-28). https://publicationethics.org/case/author-dispute-concerning-ownership-data

COPE Council. (2001). Redundant publication [01-10]. https://publicationethics.org/case/redundant-publication-1

COPE Council. (2009). COPE Guidelines for retracting articles [2009]. Committee on Publication Ethics. https://doi.org/10.24318/cope.2019.1.4

COPE Council. (2010). Falsified references (No. 10-29). https://publicationethics.org/case/falsified-references

COPE Council. (2012a). Compromised peer review system in published papers (No. 12-12). https://publicationethics.org/case/compromised-peer-review-system-published-papers

COPE Council. (2012b). Compromised peer review (unpublished) (No. 12-16). https://publicationethics.org/case/compromised-peer-review-unpublished

COPE Council. (2013). Misattributed authorship and unauthorized use of data (No. 13-17). https://publicationethics.org/case/misattributed-authorship-and-unauthorized-use-data

COPE Council. (2018). How to recognise potential authorship problems. Committee on Publication Ethics. https://doi.org/10.24318/cope.2019.2.22

COPE Council. (2019a). COPE Discussion Document: Citation Manipulation. Committee on Publication Ethics. https://doi.org/10.24318/cope.2019.3.1

COPE Council. (2019b). Retraction guidelines. http://doi.org/10.24318/cope.2019.1.4

COPE Council. (2021, February 10). COPE collaboration with FORCE11 Working Group. COPE: Committee on Publication Ethics. https://publicationethics.org/news/copecollaboration-force11-working-group

Cortegiani, A., Catalisano, G., Ippolito, M., Giarratano, A., Absalom, A. R., \& Einav, S. (2021). Retracted papers on SARS-CoV-2 and COVID-19. British Journal of Anaesthesia, 126(4), e155-e156. https://doi.org/10.1016/j.bja.2021.01.008 
Cox, A., Craig, R., \& Tourish, D. (2018). Retraction statements and research malpractice in economics. Research Policy, 47(5), 924-935. https://doi.org/10.1016/j.respol.2018.02.016

Crossref. (n.d.). Crossmark. Crossref. Retrieved November 11, 2019, from https://www.crossref.org/services/crossmark/

Cumpston, M., \& Chandler, J. (2021). Chapter IV: Updating a review. In J. Higgins, J. Thomas, J. Chandler, M. Cumpston, T. Li, M. Page, \& V. Welch (Eds.), Cochrane Handbook for Systematic Reviews of Interventions (version 6.2). Cochrane. https://training.cochrane.org/handbook/current/chapter-iv

Dafoe, A. (2014). Science deserves better: The imperative to share complete replication files. PS: Political Science \& Politics, 47(1), 60-66. https://doi.org/10.1017/S104909651300173X

Davis, P. M. (2012). The persistence of error: A study of retracted articles on the Internet and in personal libraries. Journal of the Medical Library Association: JMLA, 100(3), 184-189. https://doi.org/10.3163/1536-5050.100.3.008 (Correction published October 2012, Journal of the Medical Library Association: JMLA, 100(4), p. 329, https://doi.org/10.3163/1536-5050.100.4.027)

Decullier, E., Huot, L., Samson, G., \& Maisonneuve, H. (2013). Visibility of retractions: A crosssectional one-year study. BMC Research Notes, 6(1), 238. https://doi.org/10.1186/17560500-6-238

Decullier, E., \& Maisonneuve, H. (2018). Correcting the literature: Improvement trends seen in contents of retraction notices. BMC Research Notes, 11(1), 490. https://doi.org/10.1186/s13104-018-3576-2

Dinh, L., Sarol, J., Cheng, Y.-Y., Hsiao, T.-K., Parulian, N., \& Schneider, J. (2019). Systematic examination of pre- and post-retraction citations. Proceedings of the Association for Information Science and Technology, 56(1), 390-394. https://doi.org/10.1002/pra2.35

Dougherty, M. V. (2020). Introduction. In M. V. Dougherty (Ed.), Disguised Academic Plagiarism: A Typology and Case Studies for Researchers and Editors (pp. 1-11). Springer International Publishing. https://doi.org/10.1007/978-3-030-46711-1_1

Dubin, D. (2004). The most influential paper Gerard Salton never wrote. Library Treñds, 52(4), 748-764. http://hdl.handle.net/2142/1697

Educopia Institute. (2016). Library Publishing Curriculum. https://educopia.org/library-publishingcurriculum/

Elia, N., Wager, E., \& Tramèr, M. R. (2014). Fate of articles that warranted retraction due to ethical concerns: A descriptive cross-sectional study. PLoS ONE, 9(1), e85846. https://doi.org/10.1371/journal.pone.0085846

Elsevier. (n.d.). Article withdrawal. Retrieved June 24, 2021, from https://www.elsevier.com/about/policies/article-withdrawal

Enserink, M. (2017, June 7). How to avoid the stigma of a retracted paper? Don't call it a retraction. Science. https://doi.org/10.1126/science.aan6937

F1000Research. (n.d.). FAQs. F1000Research. Retrieved March 12, 2021, from https://f1000research.com/faqs

Faggion, C. M. (2019). More detailed guidance on the inclusion/exclusion of retracted articles in systematic reviews is needed. Journal of Clinical Epidemiology, 116, 133-134. https://doi.org/10.1016/j.jclinepi.2019.07.006

Fanelli, D. (2013). Why growing retractions are (mostly) a good sign. PLoS Medicine, 10(12), e1001563. https://doi.org/10.1371/journal.pmed.1001563

Fanelli, D. (2016). Set up a 'self-retraction' system for honest errors. Nature, 531(7595), 415. https://doi.org/10.1038/531415a

Fanelli, D., Costas, R., Fang, F. C., Casadevall, A., \& Bik, E. M. (2019). Testing hypotheses on risk factors for scientific misconduct via matched-control analysis of papers containing 
problematic image duplications. Science and Engineering Ethics, 25(3), 771-789. https://doi.org/10.1007/s11948-018-0023-7

Fang, F. C., Steen, R. G., \& Casadevall, A. (2012). Misconduct accounts for the majority of retracted scientific publications. Proceedings of the National Academy of Sciences, 109(42), 17028-17033. https://doi.org/10.1073/pnas.1212247109 (Correction published January 15, 2013, Proceedings of the National Academy of Sciences, 110(3), p. 1137, https://doi.org/10.1073/pnas.1220649110)

Ferguson, C., Marcus, A., \& Oransky, I. (2014). Publishing: The peer-review scam. Nature News, 515(7528), 480. https://doi.org/10.1038/515480a

Fernández, L. M., \& Vadillo, M. A. (2019). Retracted papers die hard: Diederik Stapel and the enduring influence of flawed science. PsyArXiv Preprints. https://doi.org/10.31234/osf.io/cszpy

Fister, I. J., Fister, I., \& Perc, M. (2016). Toward the discovery of citation cartels in citation networks. Frontiers in Physics, 4. https://doi.org/10.3389/fphy.2016.00049

Flanagin, A., Heckner, H., Poff, D., Seguin, J., \& Schneider, J. (2021). A cross-industry discussion on retracted research: Connecting the dots for shared responsibility. Society for Scholarly Publishing 43rd Annual Meeting. https://www.ideals.illinois.edu/handle/2142/110140

Foltýnek, T., Meuschke, N., \& Gipp, B. (2019). Academic plagiarism detection: A systematic literature review. ACM Computing Surveys, 52(6), 112:1-112:42. https://doi.org/10.1145/3345317

FORCE11. (2020, November 14). Research Data Publishing Ethics. FORCE11. https://www.force11.org/group/research-data-publishing-ethics

Fu, Y., \& Schneider, J. (2020). Towards knowledge maintenance in scientific digital libraries with the keystone framework. Proceedings of the ACM/IEEE Joint Conference on Digital Libraries in 2020 (JCDL '20), 217-226. https://doi.org/10.1145/3383583.3398514

Fu, Y., Schneider, J., \& Blake, C. (2021). Finding keystone citations for constructing validity chains among research papers. WWW'21: Companion Proceedings of the Web Conference 2021, 451-455. https://doi.org/10.1145/3442442.3451368

Fulton, A. S., Coates, A. M., Williams, M. T., Howe, P. R. C., \& Hill, A. M. (2015). Persistent citation of the only published randomised controlled trial of Omega-3 supplementation in Chronic Obstructive Pulmonary Disease six years after its retraction. Publications, 3(1), 7-26. https://doi.org/10.3390/publications3010017 (Correction published July 27, 2018, Publications, 6(3), p. 35, https://doi.org/10.3390/publications6030035)

Gabehart, M. E. (2005). An analysis of citations to retracted articles in the scientific literature [Master's, University of North Carolina at Chapel Hill]. https://ils.unc.edu/MSpapers/3050.pdf

Garfield, E. (1965). Can citation indexing be automated? Statistical Association Methods for Mechanized Documentation, Symposium Proceedings, 269, 189-192. http://garfield.library.upenn.edu/essays/V1p084y1962-73.pdf

Gewin, V. (2014). Retractions: A clean slate. Nature, 507(7492), 389-391. https://doi.org/10.1038/nj7492-389a

Gray, R., Al-Ghareeb, A., Davis, J., McKenna, L., \& Amichai Hillel, S. (2018). Inclusion of nursing trials in systematic reviews after they have been retracted: Does it happen and what should we do? International Journal of Nursing Studies, 79, 154. https://doi.org/10.1016/j.ijnurstu.2017.12.006

Gray, R., Al-Ghareeb, A., \& McKenna, L. (2019). Why articles continue to be cited after they have been retracted: An audit of retraction notices. International Journal of Nursing Studies, 90, 11-12. https://doi.org/10.1016/j.ijnurstu.2018.10.003 
Grieneisen, M. L., \& Zhang, M. (2012). A comprehensive survey of retracted articles from the scholarly literature. PLOS ONE, 7(10), e44118.

https://doi.org/10.1371/journal.pone.0044118

Habicher, M., Perrino, A. C., Spies, C., Heymann, C. von, Wittkowski, U., \& Sander, M. (2011). Retractions lead to revision of review article "Contemporary fluid management in cardiac anesthesia." Journal of Cardiothoracic and Vascular Anesthesia, 25(6), e55. https://doi.org/10.1053/j.jvca.2011.06.026

Halevi, G. (2020). Why articles in arts and humanities are being retracted? Publishing Research Quarterly, 36, 55-62. https://doi.org/10.1007/s12109-019-09699-9

Hamilton, D. G. (2019). Continued citation of retracted radiation oncology literature-Do we have a problem? International Journal of Radiation Oncology•Biology•Physics, 103(5), 1036-1042. https://doi.org/10.1016/j.jjobp.2018.11.014

Haustein, S., Bowman, T. D., \& Costas, R. (2015). When is an article actually published? An analysis of online availability, publication, and indexation dates. Proceedings of ISSI 2015 Istanbul: 15th International Society of Scientometrics and Informetrics Conference, Istanbul, Turkey, 29 June to 3 July, 2015, 1170-1179. https://www.issisociety.org/proceedings/issi_2015/1170.pdf

$\mathrm{He}, \mathrm{T}$. (2013). Retraction of global scientific publications from 2001 to 2010 . Scientometrics, 96(2), 555-561. https://doi.org/10.1007/s11192-012-0906-3

Heckers, S., Bauchner, H., \& Flanagin, A. (2015). Retracting, replacing, and correcting the literature for pervasive error in which the results change but the underlying science is still reliable. JAMA Psychiatry, 72(12), 1170.

https://doi.org/10.1001/jamapsychiatry.2015.2278

Hesselmann, F., \& Reinhart, M. (2019). Science means never having to say you're sorry? Apologies for scientific misconduct. Science Communication, 41(5), 552-579. https://doi.org/10.1177/1075547019860848

Horbach, S. P. J. M., \& Halffman, W. (2017). The ghosts of HeLa: How cell line misidentification contaminates the scientific literature. PLOS ONE, 12(10), e0186281. https://doi.org/10.1371/journal.pone.0186281

Horton, J., Krishna Kumar, D., \& Wood, A. (2020). Detecting academic fraud using Benford law: The case of Professor James Hunton. Research Policy, 49(8), 104084. https://doi.org/10.1016/j.respol.2020.104084

Hosseini, M., Hilhorst, M., de Beaufort, I., \& Fanelli, D. (2018). Doing the right thing: A qualitative investigation of retractions due to unintentional error. Science and Engineering Ethics, 24(1), 189-206. https://doi.org/10.1007/s11948-017-9894-2

Hovaguimian, F., Elia, N., \& Tramèr, M. R. (2019). Supplemental oxygen and the risk of surgical site infection: Evidence of compromised data requires correction of previously published meta-analysis. Anesthesiology, 131(4), 932-933. https://doi.org/10.1097/ALN.0000000000002897

Hsiao, T.-K., \& Schneider, J. (2021). Continued use of retracted papers: Temporal trends in citations and (lack of) awareness of retractions shown in citation contexts in biomedicine [manuscript]. https://osf.io/4jexb/

Hu, G., \& Xu, S. (Brian). (2020). Agency and responsibility: A linguistic analysis of culpable acts in retraction notices. Lingua, 102954. https://doi.org/10.1016/j.lingua.2020.102954

Huh, S., Kim, S. Y., \& Cho, H.-M. (2016). Characteristics of retractions from Korean Medical Journals in the KoreaMed database: A bibliometric analysis. PLOS ONE, 11(10), e0163588. https://doi.org/10.1371/journal.pone.0163588

International Committee of Medical Journal Editors. (2019). Recommendations for the conduct, reporting, editing, and publication of scholarly work in medical journals. http://www.icmje.org/icmje-recommendations.pdf 
International Committee of Medical Journal Editors. (2021). Journals stating that they follow the ICMJE Recommendations. International Committee of Medical Journal Editors. http://www.icmje.org/journals-following-the-icmje-recommendations/

Jan, R., \& Zainab, T. (2018). The impact story of retracted articles: Altmetric it! 2018 th International Symposium on Emerging Trends and Technologies in Libraries and Information Services (ETTLIS), 402-406. https://doi.org/10.1109/ETTLIS.2018.8485245

Jolly, B. L. K., Jain, L., Bera, D., \& Chakraborty, T. (2020). Unsupervised anomaly detection in journal-level citation networks. Proceedings of the ACM/IEEE Joint Conference on Digital Libraries in 2020, 27-36. https://doi.org/10.1145/3383583.3398531

Kane, M., \& Trochim, W. (2009). Concept mapping for applied social research. In The SAGE Handbook of Applied Social Research Methods (2nd ed., pp. 435-474). SAGE Publications, Inc. https://doi.org/10.4135/9781483348858

Kim, S. Y., Yi, H. J., Cho, H.-M., \& Huh, S. (2019). How many retracted articles indexed in KoreaMed were cited 1 year after retraction notification. Science Editing, 6(2), 122-127. https://doi.org/10.6087/kcse.172

Kochan, C. A., \& Budd, J. M. (1992). The persistence of fraud in the literature: The Darsee case. Journal of the American Society for Information Science, 43(7), 488-493. https://doi.org/10.1002/(SICI)1097-4571(199208)43:7<488::AID-ASI3>3.0.CO;2-7

Kodvanj, I., Homolak, J., Virag, D., \& Trkulja, V. (2020). Publishing of COVID-19 preprints in peer-reviewed journals, preprinting trends, public discussion and quality issues. BioRxiv, 2020.11.23.394577. https://doi.org/10.1101/2020.11.23.394577

Korpela, K. M. (2010). How long does it take for the scientific literature to purge itself of fraudulent material?: The Breuning case revisited. Current Medical Research and Opinion, 26(4), 843-847. https://doi.org/10.1185/03007991003603804

Koziol, M. (2016, August 10). You've been dupe'd: Results so nice, journals published them twice. Retraction Watch. https://retractionwatch.com/2016/08/10/youve-been-dupedresults-so-nice-the-journals-published-them-twice/

Kullgren, K. A., \& Carter, B. D. (2015). Retraction experience, lessons learned, and recommendations for clinician researchers. Clinical Practice in Pediatric Psychology, 3(4), 352-357. https://doi.org/10.1037/cpp0000120

Labbé, C., Cabanac, G., West, R. A., Gautier, T., Favier, B., \& Byrne, J. A. (2020). Flagging incorrect nucleotide sequence reagents in biomedical papers: To what extent does the leading publication format impede automatic error detection? Scientometrics, 124(2), 1139-1156. https://doi.org/10.1007/s11192-020-03463-z

Li, E. C., Heran, B. S., \& Wright, J. M. (2014). Angiotensin converting enzyme (ACE) inhibitors versus angiotensin receptor blockers for primary hypertension. Cochrane Database of Systematic Reviews, 8. https://doi.org/10.1002/14651858.CD009096.pub2

Linger, R., \& Graham, D. (2014). A common case of cell line misidentification. The Winnower. https://doi.org/10.15200/winn.140076.66222

Lu, S. F., Jin, G. Z., Uzzi, B., \& Jones, B. (2013). The retraction penalty: Evidence from the Web of Science. Scientific Reports, 3, 3146. https://doi.org/10.1038/srep03146

Mann, R. P. (2017). Viewpoint from own experience of retraction [Oral Presentation]. The World Conference on Research Integrity 2017, Amsterdam. https://wcrif.org/images/2017/documents/1.\%20Monday\%20May\%2029,\%202017/1.\%20 Aula/R.\%20Mann\%20\%20Viewpoint\%20from\%20own\%20experience\%20of\%20retraction.pdf

Marasović, T., Utrobiĉić, A., \& Maruŝić, A. (2018). Transparency of retracting and replacing articles. The Lancet, 391(10127), 1244-1245. https://doi.org/10.1016/S01406736(18)30487-2 
Marcus, A. (2018, June 19). Fecal transplant paper pulled for "personal issue." Retraction Watch. https://retractionwatch.com/2018/06/19/fecal-transplant-paper-pulled-forpersonal-issue/

Marcus, A. (2020, December 9). Authors of meta-analysis on heart disease retract it when they realize a NEJM reference had been retracted. Retraction Watch. https://retractionwatch.com/2020/12/09/authors-of-meta-analysis-on-heart-diseaseretract-it-when-they-realize-a-nejm-reference-had-been-retracted/

Marcus, A. (2021, April 13). One in six of the papers you cite in a review has been retracted. What do you do? Retraction Watch. https://retractionwatch.com/2021/04/13/one-in-sixof-the-papers-you-cite-in-a-review-has-been-retracted-what-do-you-do/

Markowitz, D. M., \& Hancock, J. T. (2016). Linguistic obfuscation in fraudulent science. Journal of Language and Social Psychology, 35(4), 435-445. https://doi.org/10.1177/0261927X15614605

Matas, J., Buljan, I., \& Marušić, A. (2020). Correcting the pandemic: Analysis of corrections to journal articles on COVID-19 and Ebola. Journal of Global Health, 10(2), 020105. https://doi.org/10.7189/jogh.10.020105

McCook, A. (2017, August 9). Elsevier retracts entire issue after mistakenly publishing it online. Retraction Watch. https://retractionwatch.com/2017/08/09/elsevier-retracts-entire-issuemistakenly-publishing-online/

McHugh, U. M., \& Yentis, S. M. (2019). An analysis of retractions of papers authored by Scott Reuben, Joachim Boldt and Yoshitaka Fujii. Anaesthesia, 74(1), 17-21. https://doi.org/10.1111/anae.14414 (Correction published December 4, 2018, Anaesthesia, 74(1), p. 113, https://doi.org/10.1111/anae.14515)

McLean, H. (2013). Legal issues in retractions \& corrections [oral presentation]. COPE Asia Pacific Seminar. https://publicationethics.org/resources/seminars/legal-issuesretractions-and-corrections-\%E2\%80\%94-helen-mclean-legal-counsel-john-wiley

Meddings, K. (2020, March 30). Encouraging even greater reporting of corrections and retractions. Crossref. https://www.crossref.org/blog/encouraging-even-greater-reportingof-corrections-and-retractions/

Mena, J. D., Ndoye, M., Cohen, A. J., Kamal, P., \& Breyer, B. N. (2019). The landscape of urological retractions: The prevalence of reported research misconduct. BJU International, 124(1), 174-179. https://doi.org/10.1111/bju.14706

Mongeon, P., \& Larivière, V. (2016). Costly collaborations: The impact of scientific fraud on coauthors' careers. Journal of the Association for Information Science and Technology, 67(3), 535-542. https://doi.org/10.1002/asi.23421

Mott, A., Fairhurst, C., \& Torgerson, D. (2019). Assessing the impact of retraction on the citation of randomized controlled trial reports: An interrupted time-series analysis. Journal of Health Services Research \& Policy, 24(1), 44-51. https://doi.org/10.1177/1355819618797965

Moylan, E. C., \& Kowalczuk, M. K. (2016). Why articles are retracted- a retrospective crosssectional study of retraction notices at BioMed Central. BMJ Open, 6(11), e012047. https://doi.org/10.1136/bmjopen-2016-012047

Naik, G. (2011, August 10). Mistakes in scientific studies surge. Wall Street Journal. https://www.wsj.com/articles/SB10001424052702303627104576411850666582080

Nath, S. B., Marcus, S. C., \& Druss, B. G. (2006). Retractions in the research literature: Misconduct or mistakes? Medical Journal of Australia, 185(3), 152-154. https://doi.org/10.5694/j.1326-5377.2006.tb00504.x

National Heart Foundation of Australia \& National Blood Pressure and Vascular Disease Advisory Committee. (2016). Guideline for the diagnosis and management of hypertension in adults (PRO-167). National Heart Foundation of Australia. 
https://www.heartfoundation.org.au/getmedia/c83511ab-835a-4fcf-96f5-

88d770582ddc/PRO-167_Hypertension-guideline-2016_WEB.pdf

National Institute for Health and Care Excellence. (2019). Hypertension in adults: Diagnosis and management (NICE Guideline No. NG136). National Institute for Health and Care Excellence. https://www.nice.org.uk/guidance/ng136

Neale, A. V., Dailey, R. K., \& Abrams, J. (2010). Analysis of citations to biomedical articles affected by scientific misconduct. Science and Engineering Ethics, 16(2), 251-261. https://doi.org/10.1007/s11948-009-9151-4

Neale, A. V., Northrup, J., Dailey, R., Marks, E., \& Abrams, J. (2007). Correction and use of biomedical literature affected by scientific misconduct. Science and Engineering Ethics, 13, 5-24. https://doi.org/10.1007/s11948-006-0003-1

Nelson, L. D., Simmons, J., \& Simonsohn, U. (2018). Psychology's Renaissance. Annual Review of Psychology, 69(1), 511-534. https://doi.org/10.1146/annurev-psych-122216011836

NISO. (2008). NISO-RP-8-2008, Journal Article Versions (JAV): Recommendations (Standard NISO-RP-8-2008; p. 37). http://www.niso.org/sites/default/files/2017-08/RP-8-2008.pdf

NISO PIE-J Working Group. (2013). The Presentation \& Identification of E-Journals (Recommended Practice NISO RP-16-2013 PIE-J). NISO. https://www.niso.org/publications/rp-16-2013-pie-j

Oransky, I. (2012, July 5). A first? Papers retracted for citation manipulation. Retraction Watch. https://retractionwatch.com/2012/07/05/a-first-papers-retracted-for-citation-manipulation/

Oransky, I. (2013, February 25). Is an "article in press" "published?" A word about Elsevier's withdrawal policy. Retraction Watch. https://retractionwatch.com/2013/02/25/is-anarticle-in-press-published-a-word-about-elseviers-withdrawal-policy/

Oransky, I. (2014, July 8). SAGE Publications busts "peer review and citation ring," 60 papers retracted. Retraction Watch. https://retractionwatch.com/2014/07/08/sage-publicationsbusts-peer-review-and-citation-ring-60-papers-retracted/

Oransky, I. (2015, May 21). What should an ideal retraction notice look like? Retraction Watch. https://retractionwatch.com/2015/05/21/what-should-an-ideal-retraction-notice-look-like/

Oransky, I. (2018a, January 5). Ask Retraction Watch: Is it OK to cite a retracted paper? Retraction Watch. https://retractionwatch.com/2018/01/05/ask-retraction-watch-ok-citeretracted-paper/

Oransky, I. (2018b, August 1). Have retraction notices improved over time? Retraction Watch. https://retractionwatch.com/2018/08/01/have-retraction-notices-improved-over-time/

Oransky, I. (2019, September 12). A publisher wants to destigmatize retractions. Here's how. Retraction Watch. https://retractionwatch.com/2019/09/12/a-journal-wants-todestigmatize-retractions-heres-how/

Oransky, I., \& Marcus, A. (2018, October 14). Harvard and the Brigham call for 31 retractions of cardiac stem cell research. STAT. https://www.statnews.com/2018/10/14/harvardbrigham-retractions-stem-cell/

Osman, E., Alnaib, Z., \& Kumar, N. (2017). Photodynamic diagnosis in upper urinary tract urothelial carcinoma: A systematic review. Arab Journal of Urology, 15(2), 100-109. https://doi.org/10.1016/j.aju.2017.01.003

Palacio, M., Kühnert, M., Berger, R., Larios, C. L., \& Marcellin, L. (2014). Meta-analysis of studies on biochemical marker tests for the diagnosis of premature rupture of membranes: Comparison of performance indexes. BMC Pregnancy and Childbirth, 14, 183-183. https://doi.org/10.1186/1471-2393-14-183

Palestis, B., Trivers, R., \& Zaatari, D. (2014). Symmetry and dance: A case of scientific fraud. The Winnower. https://doi.org/10.15200/winn.140076.67602

Palus, A. S. (2015, December 8). Diederik Stapel now has 58 retractions. Retraction Watch. https://retractionwatch.com/2015/12/08/diederik-stapel-now-has-58-retractions/ 
Pandit, J. J. (2012). On statistical methods to test if sampling in trials is genuinely random. Anaesthesia, 67(5), 456-462. https://doi.org/10.1111/j.1365-2044.2012.07114.x

Pantziarka, P., \& Meheus, L. (2019). Journal retractions in oncology: A bibliometric study. Future Oncology, 15(31), 3597-3608. https://doi.org/10.2217/fon-2019-0233

Patrias, K., \& Wendling, D. (2018). Citing Medicine (2nd ed.). National Library of Medicine (US).

Pfeifer, M. P., \& Snodgrass, G. L. (1990). The continued use of retracted, invalid scientific literature. The Journal of the American Medical Association, 263(10), 1420-1423. https://doi.org/10.1001/jama.1990.03440100140020

Piller, C. (2021). Disgraced COVID-19 studies are still routinely cited. Science, 371(6527), 331332. https://doi.org/10.1126/science.371.6527.331

Proescholdt, R. (2021). RISRS Retraction Review_Field Variation Data. University of Illinois at Urbana-Champaign. https://doi.org/10.13012/B2IDB-2070560_V1

Proescholdt, R., \& Schneider, J. (2020, October 22). Retracted papers with inconsistent document type indexing in PubMed, Scopus, and Web of Science [poster]. METRICS 2020 workshop at ASIS\&T 2020. http://hdl.handle.net/2142/110134

RedacTek. (2021). How it works. https://release.redactek.com/how-it-works/

Redman, B. K., Yarandi, H. N., \& Merz, J. F. (2008). Empirical developments in retraction. Journal of Medical Ethics, 34(11), 807-809. https://doi.org/10.1136/jme.2007.023069

Reinhart, K., \& Takala, J. (2011). Hydroxyethyl starches: What do we still know? Anesthesia \& Analgesia, 112(3), 507-511. https://doi.org/10.1213/ANE.0b013e3182099c49

Resnik, Jr., D. B., \& Stewart Jr., N. (2012). Misconduct versus honest error and scientific disagreement. Accountability in Research, 19(1), 56-63. https://doi.org/10.1080/08989621.2012.650948

Retraction Watch. (n.d.-a). Citation manipulation. Retraction Watch. Retrieved April 24, 2021, from https://retractionwatch.com/category/by-reason-for-retraction/citation-manipulation/

Retraction Watch. (n.d.-b). Doing the right thing. Retraction Watch. Retrieved March 5, 2021, from https://retractionwatch.com/category/by-reason-for-retraction/doing-the-right-thing/

Retraction Watch. (n.d.-c). Lack of IRB approval. Retraction Watch. Retrieved April 24, 2021, from https://retractionwatch.com/category/by-reason-for-retraction/lack-of-irb-approval/

Retraction Watch. (n.d.-d). Retraction Watch. Retrieved March 11, 2021, from https://retractionwatch.com/

RISRS Bibliography v.1. (2021). InfoQualityLab. https://github.com/infoqualitylab/EPPIReviewer_to_Exhibit_JSON

RISRS Bibliography v.2.15. (2021). InfoQualityLab. https://github.com/infoqualitylab/EPPIReviewer_to_Exhibit_JSON/releases/tag/2.15

Rohrer, J. M., Tierney, W., Uhlmann, E. L., DeBruine, L. M., Heyman, T., Jones, B., Schmukle, S. C., Silberzahn, R., Willén, R. M., Carlsson, R., Lucas, R. E., Strand, J., Vazire, S., Witt, J. K., Zentall, T. R., Chabris, C. F., \& Yarkoni, T. (2021). Putting the Self in SelfCorrection: Findings From the Loss-of-Confidence Project. Perspectives on Psychological Science, 1745691620964106. https://doi.org/10.1177/1745691620964106

Ronald, P. (2013, October 10). Lab life: The anatomy of a retraction. Scientific American Blog Network. https://blogs.scientificamerican.com/food-matters/lab-life-the-anatomy-of-aretraction/

Saikia, P., \& Thakuria, B. (2019). Retraction of papers authored by Yuhji Saitoh - Beyond the Fujii phenomenon. Indian Journal of Anaesthesia, 63(7), 571-584. https://doi.org/10.4103/ija.IJA_267_19

Santos-d'Amorim, K., Melo, R. R. de, \& Santos, R. N. M. dos. (2021). Retractions and postretraction citations in the COVID-19 infodemic: Is Academia spreading misinformation? [Retratações e citações pós-retratação na infodemia de COVID-19: a Academia está espalhando desinformação?]. Liinc Em Revista, 17(1), e5593-e5593.

https://doi.org/10.18617/liinc.v17i1.5593 
Sarol, M. J., Liu, L., \& Schneider, J. (2018). Testing a citation and text-based framework for retrieving publications for literature reviews. Proceedings of the 7th International Workshop on Bibliometric-Enhanced Information Retrieval (BIR 2018) Co-Located with the 40th European Conference on Information Retrieval (ECIR 2018), 22-33. http://ceurws.org/Vol-2080/paper3.pdf

Schmidt, M. (2018). An analysis of the validity of retraction annotation in PubMed and the Web of Science. Journal of the Association for Information Science and Technology, 69(2), 318-328. https://doi.org/10.1002/asi.23913

Schneider, J., Ye, D., Hill, A. M., \& Whitehorn, A. S. (2020). Continued post-retraction citation of a fraudulent clinical trial report, 11 years after it was retracted for falsifying data. Scientometrics, 125(3), 2877-2913. https://doi.org/10.1007/s11192-020-03631-1

Sehnal, D., Svobodová Vařeková, R., Pravda, L., lonescu, C.-M., Geidl, S., Horský, V., Jaiswal, D., Wimmerová, M., \& Koča, J. (2015). ValidatorDB: database of up-to-date validation results for ligands and non-standard residues from the Protein Data Bank. Nucleic Acids Research, 43(D1), D369-D375. https://doi.org/10.1093/nar/gku1118

Seife, C. (2015). Research misconduct identified by the US Food and Drug Administration: Out of sight, out of mind, out of the peer-reviewed literature. JAMA Internal Medicine, 175(4), 567-577. https://doi.org/10.1001/jamainternmed.2014.7774

Sheehan, A. H., \& Beckett, R. D. (2016). Recently retracted clinical trial serves as an important reminder. Annals of Pharmacotherapy. https://doi.org/10.1177/1060028016630761

Shema, H., Hahn, O., Mazarakis, A., \& Peters, I. (2019). Retractions from altmetric and bibliometric perspectives. Information - Wissenschaft \& Praxis, 70(2-3), 98-110. https://doi.org/10.1515/iwp-2019-2006

Shuai, X., Rollins, J., Moulinier, I., Custis, T., Edmunds, M., \& Schilder, F. (2017). A multidimensional investigation of the effects of publication retraction on scholarly impact. Journal of the Association for Information Science and Technology, 68(9), 2225-2236. https://doi.org/10.1002/asi.23826

Simkin, M. V., \& Roychowdhury, V. P. (2005). Stochastic modeling of citation slips. Scientometrics, 62(3), 367-384. https://doi.org/10.1007/s11192-005-0028-2

Snodgrass, G. L., \& Pfeifer, M. P. (1992). The characteristics of medical retraction notices. Bulletin of the Medical Library Association, 80(4), 328-334. https://www.ncbi.nlm.nih.gov/pubmed/1422502

Souder, L. (2010). A rhetorical analysis of apologies for scientific misconduct: Do they really mean it? Science and Engineering Ethics, 16(1), 175-184. https://doi.org/10.1007/s11948-009-9149-y

Stapel, D. (2014). Trying to fix it. The Winnower. https://doi.org/10.15200/winn.140076.66506

Stavale, R., Ferreira, G. I., Galvão, J. A. M., Zicker, F., Novaes, M. R. C. G., Oliveira, C. M. de, \& Guilhem, D. (2019). Research misconduct in health and life sciences research: A systematic review of retracted literature from Brazilian institutions. PLOS ONE, 14(4), e0214272. https://doi.org/10.1371/journal.pone.0214272

Steen, R. G. (2011). Retractions in the medical literature: How many patients are put at risk by flawed research? Journal of Medical Ethics, 37(11), 688-692. https://doi.org/10.1136/jme.2011.043133

Steen, R. G. (2012). Retractions in the medical literature: How can patients be protected from risk? Journal of Medical Ethics, 38(4), 228-232. http://www.jstor.org/stable/23215468

Steen, R. G., Casadevall, A., \& Fang, F. C. (2013). Why has the number of scientific retractions increased? PLOS ONE, 8(7), e68397. https://doi.org/10.1371/journal.pone.0068397 (Correction published July 19, 2013, PLOS ONE, 8(7), https://doi.org/10.1371/annotation/0d28db18-e117-4804-b1bc-e2da285103ac) 
Stern, A. M., Casadevall, A., Steen, R. G., \& Fang, F. C. (2014). Financial costs and personal consequences of research misconduct resulting in retracted publications. ELife, 3. https://doi.org/10.7554/eLife.02956

Stern, V. (2017, September 21). Surgery journal retracts two papers it didn't mean to publish. Retraction Watch. https://retractionwatch.com/2017/09/21/surgery-journal-retracts-twopapers-didnt-mean-publish/

Stern, V. (2018, April 12). Authors retract heart disease paper for "nonscientific reason." Retraction Watch. https://retractionwatch.com/2018/04/12/authors-retract-heart-diseasepaper-for-nonscientific-reason/

Stillman, D. (2019, June 14). Retracted item notifications with Retraction Watch integration. Zotero Blog. https://www.zotero.org/blog/retracted-item-notifications/

Strand, J. (2020, April 15). Scientists make mistakes. I made a big one. Medium. https://elemental.medium.com/when-science-needs-self-correcting-a130eacb4235

Suelzer, E. M., Deal, J., \& Hanus, K. L. (2020). Challenges in discovering the retracted status of an article [manuscript]. Discussion paper for Reducing the Inadvertent Spread of Retracted Science: Shaping a Research and Implementation Agenda. http://hdl.handle.net/2142/108367

Suelzer, E. M., Deal, J., Hanus, K. L., Ruggeri, B., Sieracki, R., \& Witkowski, E. (2019). Assessment of citations of the retracted article by Wakefield et al with fraudulent claims of an association between vaccination and autism. JAMA Network Open, 2(11), e1915552. https://doi.org/10.1001/jamanetworkopen.2019.15552

Suelzer, E. M., Deal, J., Hanus, K., Ruggeri, B. E., \& Witkowski, E. (2021). Challenges in identifying the retracted status of an article. JAMA Network Open, 4(6), e2115648e2115648. https://doi.org/10.1001/jamanetworkopen.2021.15648

The Editors of The Lancet. (2009). Retraction-Combination treatment of angiotensin-II receptor blocker and angiotensin-converting-enzyme inhibitor in non-diabetic renal disease (COOPERATE): A randomised controlled trial. The Lancet, 374(9697), 1226. https://doi.org/10.1016/S0140-6736(09)61768-2

The Editors of The Lancet. (2015). Correcting the scientific literature: Retraction and republication. The Lancet, 385(9966), 394. https://doi.org/10.1016/S01406736(15)60137-4

The European Association of Science Editors. (2021). EASE Standard Retraction Form. https://ease.org.uk/publications/ease-statements-resources/ease-standard-retractionform/

Thomas Manapurathe, D., Krishna, S. M., Dewdney, B., Moxon, J. V., Biros, E., \& Golledge, J. (2017). Effect of blood pressure lowering medications on leg ischemia in peripheral artery disease patients: A meta-analysis of randomised controlled trials. PloS One, 12(6), e0178713. PubMed. https://doi.org/10.1371/journal.pone.0178713

Tort, A. B. L., Targino, Z. H., \& Amaral, O. B. (2012). Rising publication delays inflate journal impact factors. PLOS ONE, 7(12), e53374. https://doi.org/10.1371/journal.pone.0053374

Tourish, D., \& Craig, R. (2020). Research misconduct in business and management studies: Causes, consequences, and possible remedies. Journal of Management Inquiry, 29(2), 174-187. https://doi.org/10.1177/1056492618792621

Trikalinos, N. A., Evangelou, E., \& loannidis, J. P. A. (2008). Falsified papers in high-impact journals were slow to retract and indistinguishable from nonfraudulent papers. Journal of Clinical Epidemiology, 61(5), 464-470. https://doi.org/10.1016/j.jclinepi.2007.11.019

Tripathi, M., Dwivedi, G., Sonkar, S. K., \& Kumar, S. (2018). Analysing retraction notices of scholarly journals: A study. DESIDOC Journal of Library \& Information Technology, 38(5), 305-311. https://doi.org/10.14429/djlit.38.5.13103 
Tripathi, M., Sonkar, S. K., \& Kumar, S. (2019). A cross sectional study of retraction notices of scholarly journals of science. DESIDOC Journal of Library \& Information Technology, 39(2), 74-81. https://doi.org/10.14429/djlit.39.2.14000

U.S. National Library of Medicine. (n.d.). Participation Agreements and Options. Retrieved March 10, 2021, from https://www.ncbi.nlm.nih.gov/pmc/pub/agreements/

U.S. National Library of Medicine. (2018, August 8). Errata, Retractions, and Other Linked Citations in PubMed [FAQs, Help Files, Pocket Cards]. https://www.nlm.nih.gov/bsd/policy/errata.html

U.S. National Library of Medicine. (2021, June 21). XML Help for PubMed Data Providers. PubMed Help [Internet]. https://www.ncbi.nlm.nih.gov/books/NBK3828/

van der Vet, P. E., \& Nijveen, H. (2016). Propagation of errors in citation networks: A study involving the entire citation network of a widely cited paper published in, and later retracted from, the journal Nature. Research Integrity and Peer Review, 1, 3. https://doi.org/10.1186/s41073-016-0008-5

Van der Walt, W., Willems, K. A., Friedrich, W., Hatsu, S., \& Krauss, K. (2020). Retracted COVID-19 Papers and the level of "citation pollution": A preliminary analysis and directions for future research. Cahiers de La Documentation - Bladen Voor Documentatie, 3-4, 206-218. http://hdl.handle.net/10962/167732

Vaughan, L., Glänzel, W., Korch, C., \& Capes-Davis, A. (2017). Widespread use of misidentified cell line KB (HeLa): Incorrect attribution and its impact revealed through mining the scientific literature. Cancer Research, 77(11), 2784-2788. https://doi.org/10.1158/00085472.CAN-16-2258

Vuong, Q.-H. (2020). The limitations of retraction notices and the heroic acts of authors who correct the scholarly record: An analysis of retractions of papers published from 1975 to 2019. Learned Publishing, 33(2), 119-130. https://doi.org/10.1002/leap.1282

Vuong, Q.-H., La, V.-P., Ho, M.-T., Vuong, T.-T., \& Ho, M.-T. (2020). Characteristics of retracted articles based on retraction data from online sources through February 2019. Science Editing, 7(1), 34-44. https://doi.org/10.6087/kcse.187

Wager, E., Kleinert, S., Bähr, V., Bazdaric, K., Farthing, M., Garfinkel, M., Graf, C., Hammatt, Z., Horn, L., King, S., Parrish, D., Pulverer, B., Taylor, P., van Meer, G., \& on behalf of the CLUE Working Group. (2021). Cooperation \& Liaison between Universities \& Editors (CLUE): Recommendations on best practice. Research Integrity and Peer Review, 6(1), 6. https://doi.org/10.1186/s41073-021-00109-3

Wager, E., \& Williams, P. (2011). Why and how do journals retract articles? An analysis of Medline retractions 1988-2008. Journal of Medical Ethics, 37(9), 567-570. https://doi.org/10.1136/jme.2010.040964

Wasiak, J., George Hamilton, D., Foroudi, F., \& Faggion, C. M. (2018). Surveying retracted studies and notices within the field of radiation oncology. International Journal of Radiation Oncology, Biology, Physics, 102(3), 660-665. https://doi.org/10.1016/j.jjobp.2018.06.028

Weise, W. J. (2003). RETRACTED: Combination ACE inhibitor and angiotensin receptor blocker therapy was better than monotherapy in non-diabetic renal disease. ACP Journal Club, 139(2), 40. https://doi.org/10.7326/ACPJC-2003-139-2-040 (Retraction published December 15, 2009, Annals of Internal Medicine, 151(12), p. 897, https://doi.org/10.7326/0003-4819-151-12-200912150-00022)

Wetterer, J. K. (2006). Quotation error, citation copying, and ant extinctions in Madeira. Scientometrics, 67(3), 351-372. https://doi.org/10.1556/Scient.67.2006.3.2

When does Edifix warn me about retractions and corrections (errata)? (n.d.). Edifix. Retrieved July 11, 2021, from https://support.edifix.com/support/solutions/articles/1000080347when-does-edifix-warn-me-about-retractions-and-corrections-errata- 
White, P. F., Kehlet, H., \& Liu, S. (2009). Perioperative analgesia: What do we still know?: Anesthesia \& Analgesia, 108(5), 1364-1367. https://doi.org/10.1213/ane.0b013e3181a16835

Wiedermann, C. J. (2018). Inaction over retractions of identified fraudulent publications: Ongoing weakness in the system of scientific self-correction. Accountability in Research, 25(4), 239-253. https://doi.org/10.1080/08989621.2018.1450143

Wiley. (n.d.). Wiley's Policy for Handling Retractions, Withdrawals, and Expressions of Concern. Retrieved April 13, 2021, from https://authorservices.wiley.com/ethicsguidelines/retractions-and-expressions-of-concern.html

Williams, P., \& Wager, E. (2013). Exploring why and how journal editors retract articles: Findings from a qualitative study. Science and Engineering Ethics, 19, 1-11. https://doi.org/10.1007/s11948-011-9292-0

Wise, J. (2013). Boldt: The great pretender. BMJ, 346, f1738. https://doi.org/10.1136/bmj.f1738

Woods, N. (2021). RISRS Problems and Opportunities Dataset. Illinois Data Bank. https://doi.org/10.13012/B2IDB-2831687_V1

Wright, K., \& McDaid, C. (2011). Reporting of article retractions in bibliographic databases and online journals. Journal of the Medical Library Association: JMLA, 99(2), 164-167. https://doi.org/10.3163/1536-5050.99.2.010

Wright, N. (1991). A Citation Context Analysis of Retracted Scientific Articles (9133185) [Ph.D., University of Maryland at College Park]. ProQuest Dissertations Publishing.

Xu, S., \& Hu, G. (2018). Retraction notices: Who authored them? Publications, 6(1), 2. https://doi.org/10.3390/publications6010002

Yeo-Teh, N. S. L., \& Tang, B. L. (2021). An alarming retraction rate for scientific publications on Coronavirus Disease 2019 (COVID-19). Accountability in Research, 28(1), 47-53. https://doi.org/10.1080/08989621.2020.1782203

Zhang, M., \& Grieneisen, M. L. (2013). The impact of misconduct on the published medical and non-medical literature, and the news media. Scientometrics, 96(2), 573-587. https://doi.org/10.1007/s11192-012-0920-5 


\section{Appendix A: RISRS Project Outcomes to Date}

The RISRS Team

To accompany "Recommendations from the Reducing the Inadvertent Spread of Retracted Science: Shaping a Research and Implementation Agenda Project"

July 2021

\section{Websites}

A. Project website https://infoqualitylab.org/projects/risrs2020/

B. RISRS collection of data \& documents in the IDEALS institutional repository https://www.ideals.illinois.edu/handle/2142/108359

C. Scoping review bibliography https://infoqualitylab.org/projects/risrs2020/bibliography/

\section{Post-Workshop Dissemination Activities:}

- Jodi Schneider. "The problems of post-retraction citation - and mitigation strategies that work," NIH Bibliometrics \& Research Assessment Symposium 2020, https://www.nihlibrary.nih.gov/services/bibliometrics/bibSymp20, October 9. Recording of the talk: https://www.youtube.com/watch?v=nSMheuwAKA8\&feature=youtu $\cdot$ be $\& \mathrm{t}=38 \mathrm{~m} 51 \mathrm{~s}$ Slides: http://hdl.handle.net/2142/110180 and https://www.slideshare.net/jodischneider/the-problems-of-post-retraction-citationand-mitigation-strategies-that-workbibliometrics-amp-research-assessmentsymposium-202020201009

- Jodi Schneider. "Continued citation of bad science and what we can do about it," Invited talk, Center for Computational \& Data Science, Syracuse University School of Information Studies, Friday February 19, 2021. Slides: https://www.slideshare.net/jodischneider/continued-citation-of-bad-science-andwhat-we-can-do-about-it20210219-243064376

- Caitlin Bakker, Hannah Heckner, Randy Townsend, Jodi Schneider. "Connecting the dots: A cross-industry discussion on retracted research," Part of Misinformation and truth: from fake news to retractions to preprint, NISO Plus 2021: February 25, 2021. Recording for subscribers via https://nisoplus21.sched.com/event/2cffe14c6663f2286055a597869970a1

Slides: http://hdl.handle.net/2142/110191

- Jodi Schneider \& Nathan D. Woods. "Reducing the inadvertent spread of retracted science", COPE Newsletter post, March 10, 2021. https://publicationethics.org/news/reducing-inadvertent-spread-retracted-science

- Jodi Schneider. "Continued citation of bad science and what we can do about it," Invited talk, joint George Washington University - Children's National Health 
System Informatics Seminar Series, Tuesday April 20, 2021. Slides:

https://www.slideshare.net/jodischneider/continued-citation-of-bad-science-andwhat-we-can-do-about-it20210420

- Annette Flanagin, Hannah Heckner, Deborah Poff, John Seguin, Jodi Schneider. "A cross-industry discussion on retracted research: Connecting the dots for shared responsibility," Society for Scholarly Publishing 43rd Annual Meeting, May 26, 2021. Slides archived in http://hdl.handle.net/2142/110140

Recording for subscribers via https://43ssp.cd.pathable.com/meetings/virtual/kCxnm2KPiqBY8prKs

- How to prevent retracted science being cited - tips, ideas and suggestions Moderated by Hervé Maisonneuve and Fiona Murphy with the participation of IJsbrand Jan Aalbersberg and Jodi Schneider, European Association of Science Editors (EASE) Forum Live!, June 25, 2021.

- Letter Responding to White House Office of Science and Technology Policy "Request for Information To Improve Federal Scientific Integrity Policies" 86 FR 34064 on behalf of the Alfred P. Sloan Funded Project "Reducing the Inadvertent Spread of Retracted Science: Shaping a Research and Implementation Agenda", Planned July 27, 2021 http://hdl.handle.net/2142/110214

- COPE annual virtual seminar, September 29, 2021.

- International Society of Managing and Technical Editors (ISMTE) panel, October 12, 2021.

- Seminar for STM Innovations, December 7, 2021.

Proposed

- Charleston Conference, November 3-5, 2021.

- Medical Library Association seminar, 2022.

III. Completed Research

- Yuanxi Fu and Jodi Schneider. "Towards knowledge maintenance in scientific digital libraries with keystone citations,"In JCDL '20: Proceedings of the ACM/IEEE Joint Conference on Digital Libraries in 2020, August 2020, Pages 217-226, http://doi.org/10.1145/3383583.3398514 Slides:

http://hdl.handle.net/2142/110172

- Randi Proescholdt \& Jodi Schneider. "Retracted papers with inconsistent document type indexing in PubMed, Scopus, and Web of Science," SIGMET poster, presented October 22, 2020 at the METRICS 2020 workshop at ASIS\&T 2020. Poster \& 2 page extended abstract. http://hdl.handle.net/2142/110134

- Jodi Schneider, Di Yi, Alison M Hill, Ashley S Whitehorn. "Continued citation of a fraudulent clinical trial report, eleven years after it was retracted for falsifying data," Scientometrics. Volume 125, issue 3, December 2020, pages 2877-2913 http://doi.org/10.1007/s11192-020-03631-1

- Yuanxi Fu, Jodi Schneider, and Catherine Blake. "Keystone citations for constructing validity chains among research papers," Sci-K 2021. In WWW '21: Companion Proceedings of the Web Conference 2021, April 2021, pages 451455. http://doi.org/10.1145/3442442.3451368 Preprint: 
IV. Datasets

http://jodischneider.com/pubs/scik2021.pdf

https://www.ideals.illinois.edu/handle/2142/109734

- Tzu-Kun Hsiao \& Jodi Schneider (2021): Dataset for "Continued use of retracted papers: Temporal trends in citations and (lack of) awareness of retractions shown in citation contexts in biomedicine". Illinois Data Bank. University of Illinois at Urbana-Champaign. Version 1: https://doi.org/10.13012/B2IDB-8255619 V1

Version 2: https://doi.org/10.13012/B2IDB-8255619 V1

- Nathan Woods. (2021). RISRS Problems and Opportunities Dataset. Illinois Data Bank. University of Illinois at Urbana-Champaign. https://doi.org/10.13012/B2IDB-2831687 V1

- Randi Proescholdt, Jodi Schneider \& RISRS Team (2020-1). RISRS Bibliography v.1 https://github.com/infoqualitylab/EPPI-

Reviewer to Exhibit JSON/releases/tag/v.1.0.0

- Randi Proescholdt, Yoss Arianlou, Katherine Howell, Jodi Schneider \& RISRS Team (2021). RISRS Bibliography v.2 https://github.com/infoqualitylab/EPPIReviewer to Exhibit JSON/releases/tag/2.0.0

- Randi Proescholdt, Yoss Arianlou, Katherine Howell, Jodi Schneider \& RISRS Team (2021). RISRS Bibliography v.2.1 https://github.com/infoqualitylab/EPPIReviewer to Exhibit JSON/releases/tag/2.1.0

- Randi Proescholdt, Yoss Arianlou, Katherine Howell, Jodi Schneider \& RISRS Team (2021). RISRS Bibliography v.2.15 https://github.com/infoqualitylab/EPPIReviewer to Exhibit JSON/releases/tag/2.15

- Proescholdt, Randi (2021): RISRS Retraction Review - Field Variation Data. University of Illinois at Urbana-Champaign. https://doi.org/10.13012/B2IDB2070560 V1

\section{Publications In Preparation and Under Review}

- Tzu-Kun Hsiao \& Jodi Schneider. (n.d.). Continued use of retracted papers: Temporal trends in citations and (lack of) awareness of retractions shown in citation contexts in biomedicine. Submitted to Quantitative Science Studies, April 2021, resubmitted July 2021. Preprint at OSF: https://osf.io/4jexb/

- Nathan D. Woods \& Jodi Schneider. Addressing the Continued Circulation of Retracted Research as a Design Problem. Submitted to GW Journal of Ethics in Publishing, June 2021.

- Michele Avissar-Whiting, Caitlin Bakker, Hannah Heckner, Sylvain Massip, Jodi Schneider, Randy Townsend, Nathan D. Woods. Submitted to Information Services \& Use (NISO Plus Special Issue), June 2021.

\section{Other Work in Progress}

- Short version of these recommendations, targeting Research Integrity and Peer Review. 
- Scoping literature review to identify what is known about the spread of retracted science, targeting Journal of the Association for Information Science and Technology

- Proposals for future research

- Collaborations to start achieving the recommended outcomes

- Participation in an ongoing FORCE11 Research Data Publishing Ethics Working Group collaborating with COPE https://www.force11.org/group/research-data-publishing-ethics

- COPE Taxonomy Working Group

- NISO Work Item Proposal on Publisher Handling of Retractions 


\section{Appendix B: \\ Problems and Opportunities Dataset}

Nathan Woods and the RISRS Team

To accompany "Recommendations from the Reducing the Inadvertent Spread of Retracted Science: Shaping a Research and Implementation Agenda Project"

July 2021

The 143 items below were used from V1 of the scoping review; for full references see "Version 1 of the Scoping Review of Empirical Research: List of 162 Central Items", pages C7-C18

Ajiferuke (2020)

Al-Ghareeb (2018)

Almeida (2016)

Alryalat (2020)

Amos (2014)

Aspura (2018)

Atlas (2004)

Ayodele (2018)

Azoulay (2015)

Balhara (2014)

Balhara (2015)

Bar-llan (2017)

Bar-Ilan (2018)

Bilbrey (2014)

Bolboaca (2019)

Bornemann-Cimenti (2016)

Bozzo (2017)

Budd (1998)

Budd (1999)

Budd (2011)

Budd (2016)

Campos-Varela (2019)

Campos-Varela (2019)

Carey (2016)

Casadevall (2014)

Cassao (2018)

Chambers (2019)

Chauvin (2019)

Chen (2013)

Chen (2018) 
Cox (2018)

Dal-Ré (2020)

Damineni (2015)

Davis (2012)

de Almeida (2016)

Decullier (2013)

Decullier (2014)

Deculllier (2018)

Dinh (2019)

Duggar (1995)

Elango (2019)

Elia (2014)

El-Tahan (2019)

Faggion (2018)

Fanelli (2013)

Fanelli (2015)

Fang (2011)

Fang (2012)

Foo (2011)

Foo (2014)

Friedman (1990)

Fulton (2015)

Furman (2012)

Gasparyan (2014)

Greitemeyer (2014)

Greitemeyer (2015)

Grieneisen (2012)

Hagberg (2020)

Halevi (2020)

Hamilton (2019)

$\mathrm{He}$ (2013)

Hesselmann (2019)

Hosseini (2018)

Hu (2017)

Huh (2016)

Hwang (2018)

Inoue (2016)

Jan (2018)

Jones (2008)

Karabag (2016)

Kim (2019)

Kochan (1992)

Korpela (2010)

Kuroki (2018) 


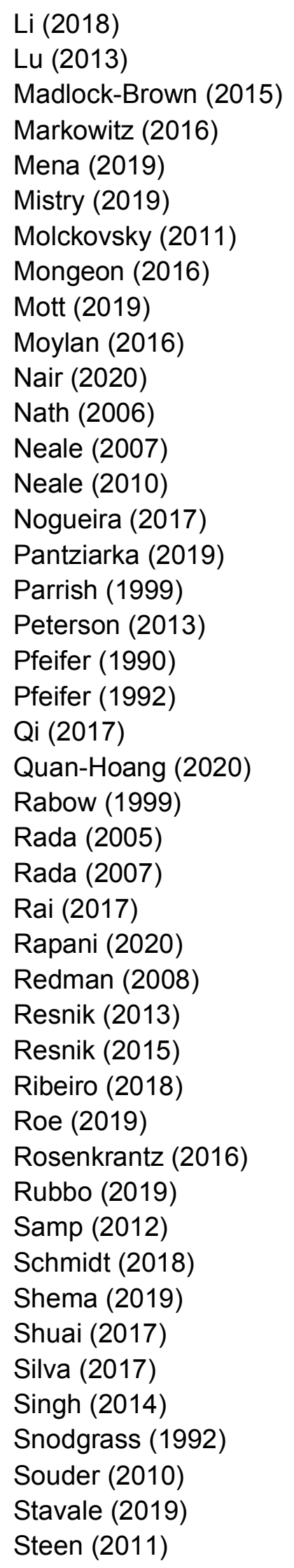


Steen (2011)

Steen (2011)

Steen (2012)

Steen (2013)

Stern (2014)

Stretton (2012)

Suelzer (2019)

Tourish (2020)

Trikalinos (2008)

Tripathi (2018)

Van Der Vet (2016)

Wager (2011)

Wang (2017)

Wang (2018)

Wasiak (2018)

Whitely (1994)

Wiedermann (2018)

Williams (2013)

Woolley (2010)

Woolley (2011)

Wray (2018)

Wright (1991)

Wright (2011)

Yan (2016)

Zhang (2013)

\section{Associated Data Set}

Woods, Nathan; (2021) RISRS Problems and Opportunities Dataset. University of Illinois at Urbana-Champaign. https://doi.org/10.13012/B2IDB-2831687 V1

\section{CRediT Contributor Statement for Appendix B: Problems and Opportunities Dataset}

Yoss Arianlou: Investigation; Halle Burns: Investigation; Yuanxi Fu: Investigation; Pamela Nila: Investigation; M. Janina Sarol: Investigation; Jodi Schneider: Conceptualization, Investigation, Methodology, Resources, Supervision, Funding acquisition, Project administration; Nathan D. Woods: Conceptualization, Investigation, Methodology, Formal analysis, Data curation, Project administration; Di Ye: Investigation; Yee Yan 'Vivien' Yip: Investigation. 


\section{Appendix C: \\ Literature Scoping Review Methods and Intermediate Results}

Randi Proescholdt, Katherine Howell, Jodi Schneider, and the RISRS Team

To accompany "Recommendations from the Reducing the Inadvertent Spread of Retracted Science: Shaping a Research and Implementation Agenda Project"

July 2021

\section{Table of Contents}

$\begin{array}{ll}\text { Inclusion Criteria } & \text { C2 }\end{array}$

$\begin{array}{ll}\text { Search Strategy } & \text { C2 }\end{array}$

$\begin{array}{ll}\text { Additional Search Methods } & \text { C3 }\end{array}$

$\begin{array}{ll}\text { Title, Abstract, and Full-Text Screening } & \text { C3 }\end{array}$

$\begin{array}{ll}\text { Categorization Process } & \text { C4 }\end{array}$

$\begin{array}{ll}\text { Scoping Review Database Search Strings } & \text { C4 }\end{array}$

$\begin{array}{ll}\text { Search conducted on July 19, } 2018 & \text { C4 }\end{array}$

Searches conducted on April 21, 2020 and February 10, $2021 \quad$ C5

$\begin{array}{ll}\text { PRISMA Diagram } & \text { C6 }\end{array}$

Version 1 of the Scoping Review of Empirical Research: List of 162 Central Items $\quad$ C7

Version 2.15 of the Scoping Review of Empirical Research: List of 386 Central Items C20

$\begin{array}{ll}\text { Associated Data Sets } & \text { C50 }\end{array}$

CRediT Contributor Statement for Appendix C: Literature Scoping Review Methods and Intermediate Results $\quad$ C51

$\begin{array}{ll}\text { BIBLIOGRAPHY } & \text { C51 }\end{array}$ 


\section{Inclusion Criteria}

We aimed to identify as many items as reasonably possible that contained original, empirical data about retraction. Specifically, we sought items that met the following inclusion criteria:

\section{In order to be included in the final review, a publication must:}

-Include original, empirical data as a result of original research. An effort to collect and analyze the data must have been made beyond reporting information that is easily attainable from databases, previous literature, or other sources.

-Systematic reviews of retracted publications (e.g., the gathering of retracted items), as well as good quality literature reviews about retracted publications (e.g., the gathering of other works that study retraction) are included when they contain original findings resulting from analysis or synthesis of the existing literature. If literature is assembled without analysis that produces new data, we do not consider the review to be empirical.

-Be "about" retraction: a publication must directly study retraction, retracted articles, retraction notices, or closely related topics (e.g. retraction-related policies, citations of retracted articles, suggestions for reforms related to retraction) or have findings, discussion, or results directly relating to these topics.

-In some cases, a paper may be included even if retraction is not its primary topic. For example, studies about scientific misconduct are not our focus, but those studies we find that present original data about retraction as part of their discussion about scientific misconduct are included.

-Findings must be relevant to retraction in general, not just relevant to a particular example or case study. However, case studies are included when they go beyond reporting the events of the case and produce their own original data that provides new insight relevant to the conversation around retraction.

\section{Search Strategy}

\section{Our database searches aimed to:}

-Include all types of scholarly publications likely to contain original, empirical research

-Include publications from any language. We seek to translate publications not in English as needed to ascertain their relevance to the review

-Exclude retracted articles and retraction notices

-Exclude news articles, correspondence, editorials, letters, or comments*

*These publications are not within the scope of our search because they would greatly increase the number of publications to be screened and many do not contain empirical data. However, we are aware that these publications sometimes do contain original, empirical data. If these publications came to our attention by means other than our search (e.g., a news article we 
came across because it is commonly cited or someone alerted us to it) and they met our inclusion criteria, they were considered for the final review.

We searched three databases_PubMed, Scopus, and Web of Science-on July 19, 2018, and again on April 21, 2020 and February 10, 2021. See (Scoping Review Database Search Strings). We aimed to identify as many items as reasonably possible that met our inclusion criteria.

\section{Additional Search Methods}

Additionally, in June/July 2018, we used the citation- and text-based framework developed by Sarol, Liu \& Schneider (2018) to retrieve other relevant articles that cited or were cited by some of the other articles included in our review, and had related terminology in their title and abstract. Five publications were used as the "seed articles" that were used to identify other potential articles of interest via Scopus (Budd et al., 1998; Chen et al., 2013; Grieneisen \& Zhang, 2012; Hesselmann et al., 2017; Pfeifer \& Snodgrass, 1990).

We also screened previous ad-hoc bibliographies created from 2017-2018 during our team's informal reviews of retraction. For our final July 2021 version we also brought in articles found from our ongoing current awareness collection. This included items culled from article Table of Contents alerts, Google Scholar alerts, bibliographies, and ad hoc encountering of publications.

\section{Title, Abstract, and Full-Text Screening}

Results from each search were brought into our review software, EPPI-Reviewer, where they were deduplicated and screened against our inclusion criteria in several stages. We removed duplicates using EPPI-Reviewer's deduplication function, as well as manually reviewing the citation information. Then we screened the remaining items to identify those that we determined to meet our criteria. First one person (JS) reviewed the titles of the retrieved items to eliminate those that we felt confident did not meet our inclusion criteria. Those that likely met our criteria or were uncertain were abstract screened by two people, and reconciled. We eliminated articles with abstracts that clearly indicated the item was not within the scope of our review. Finally, we full-text screened the articles that we had determined might meet our criteria based on their abstracts, as well as those with no abstracts. During full-text screening, we read each article and coded it as "include" or "exclude"; subcategories were also assigned to indicate provisionally included articles (e.g., those with limited empirical data) and reasons for exclusion (e.g., those not related to retraction, or those not containing original research). At the abstract and full-text steps, articles were reviewed by at least two team members, and discussed when there was disagreement so that consensus was reached.

For items we retrieved that were data rather than a full publication, we attempted to find an associated full publication to pull in and screen in its place if we did not already have it. If we could not find a full publication, we excluded the item. For books and conference proceedings, we screened each chapter or conference paper individually in most cases, unless we deemed the work relevant as a whole or irrelevant as a whole. These items are referred to as "Proxy items" in our PRISMA diagram below. 


\section{Categorization Process}

We have placed the articles included in the review in a variety of categories. The 386 articles currently included (v2.15 of the scoping review) can be found on the project website (https://infoqualitylab.org/projects/risrs2020/bibliography/ ), and can be filtered by the categories we have assigned them related to the topics of study-authorship of retracted papers, citation of retracted papers, perceptions and discussion of retracted papers, impacts of retraction, characteristics of retraction notices, characteristics of retracted papers, database search limitations used by studies, interviews and surveys, processes and policies related to retraction, studies related to discipline or geography, visibility of retraction status, detecting and preventing problems in the literature, and other-as well as other traditional methods of filtering such as publication type. We are currently in the process of refining these categories to help with the analysis and comparison of the diverse set of publications included in this review.

\section{Scoping Review Database Search Strings}

The following search strings were used:

Search conducted on July 19, 2018

PubMed (Legacy) (216 results):

((retracted articles[Title/Abstract] OR retracted publications[Title/Abstract])) OR ("Retraction of Publication as Topic"[MeSH] NOT"Comment"[PT] NOT "News"[PT] NOT Letter[PT] NOT Editorial[PT] NOT "Retracted Publication"[PT] NOT "Retraction of Publication"[PT] NOT "Published Erratum" [PT])

Scopus (730 results)

(TITLE-ABS-KEY ( retract* AND publication ) AND NOT TITLE ( "Retraction notice to" ) AND NOT TITLE ( "Retraction to" ) AND NOT TITLE ( "Erratum notice to" ) AND NOT TITLE ( "Erratum to" ) AND NOT TITLE ( "Retraction:" ) AND NOT TITLE ( "Erratum:" ) ) AND (EXCLUDE ( DOCTYPE,"no" ) OR EXCLUDE ( DOCTYPE,"ed" ) OR EXCLUDE ( DOCTYPE,"er" ) OR EXCLUDE ( DOCTYPE,"le" ) OR EXCLUDE ( DOCTYPE,"pr" ) OR EXCLUDE ( DOCTYPE,"tb" ) )

Web of Science (181 results)

((TS=(retract NEAR publication) OR TS=(retraction NEAR article $))$ ) AND DOCUMENT TYPES: (Article OR Abstract of Publishedltem OR Bibliography OR Biographical-Item OR Book OR Book Chapter OR Chronology OR Data Paper OR Discussion OR EarlyAccess OR Excerpt OR Item About an Individual OR Meeting Abstract OR Meeting Summary OR Proceedings Paper OR ReprintOR Review) 
Refined by: [excluding] DOCUMENT TYPES: ( RETRACTION OR RETRACTED PUBLICATION )Timespan: All years. Indexes: SCI-EXPANDED, SSCl, A\&HCl, CPCl-S, CPCI-SSH, BKCl-S, BKCl-SSH, ESCI, CCR-EXPANDED,IC.

Searches conducted on April 21, 2020 and February 10, 2021

PubMed (Legacy) (2297 results as of April 21, 2020; 2518 results as of February 10, 2021)

(retract* ${ }^{*}$ Title/Abstract] AND (paper*[Title/Abstract] OR notice*[Title/Abstract] OR article*[Title/Abstract] OR publication*[Title/Abstract] OR misconduct[Title/Abstract]) OR "Retraction of Publication as Topic"[MeSH] NOT "Comment"[PT] NOT "News"[PT] NOT Letter[PT] NOT Editorial[PT] NOT "Retracted Publication"[PT] NOT "Retraction of Publication"[PT] NOT "Published Erratum" [PT])

Scopus (2040 results as of April 21, 2020; 2325 results as of February 10, 2021)

( ( TITLE-ABS-KEY ( retract* W/15 publication ) OR ABS ( retract* W/15 article ) OR ABS ( retract $^{*}$ W/15 paper ) OR KEY ( retract ${ }^{*} W / 15$ paper ) OR TITLE ( retract ${ }^{*}$ W/15 paper ) OR TITLE-ABS-KEY ( retract ${ }^{*}$ W/15 "notice" ) OR TITLE-ABS-KEY ( retract ${ }^{*}$ AND misconduct ) AND NOT TITLE ( "Retraction notice to" ) AND NOT TITLE ( "Retraction to" ) ) ) AND NOT TITLE ( "Retraction notice to" ) AND NOT TITLE ( "Retraction to" ) AND ( EXCLUDE ( DOCTYPE , "no" ) OR EXCLUDE ( DOCTYPE, "ed") OR EXCLUDE ( DOCTYPE , "er" ) OR EXCLUDE ( DOCTYPE , "le" ) OR EXCLUDE ( DOCTYPE , "pr" ) OR EXCLUDE ( DOCTYPE , "tb" ) )

Web of Science (3989 results as of April 21, 2020; 4314 results as of February 10, 2021)

(TS $=\left(\right.$ retract* ${ }^{*}$ NEAR publication $\left.\$\right)$ OR TS $=\left(\right.$ retract $^{*}$ NEAR article $\left.\$\right)$ OR TS $=\left(\right.$ retract $^{*}$ NEAR paper\$)) OR AB $=\left(\right.$ retract $^{*}$ NEAR notice $\left.\$\right)$ OR TS $=\left(\right.$ retract $^{*}$ AND misconduct $)$

Refined by: [excluding] DOCUMENT TYPES: ( RETRACTED PUBLICATION OR EDITORIAL OR CORRECTION OR PATENT OR LETTER OR RETRACTION OR NEWS OR CORRECTION ADDITION)

Timespan: All years. Databases: WOS, BIOABS, BCI, CABI, CCC, DRCI, DIIDW, FSTA, KJD, MEDLINE, RSCI, SCIELO, ZOOREC.

Search language $=$ Auto 


\section{PRISMA Diagram ${ }^{1}$}

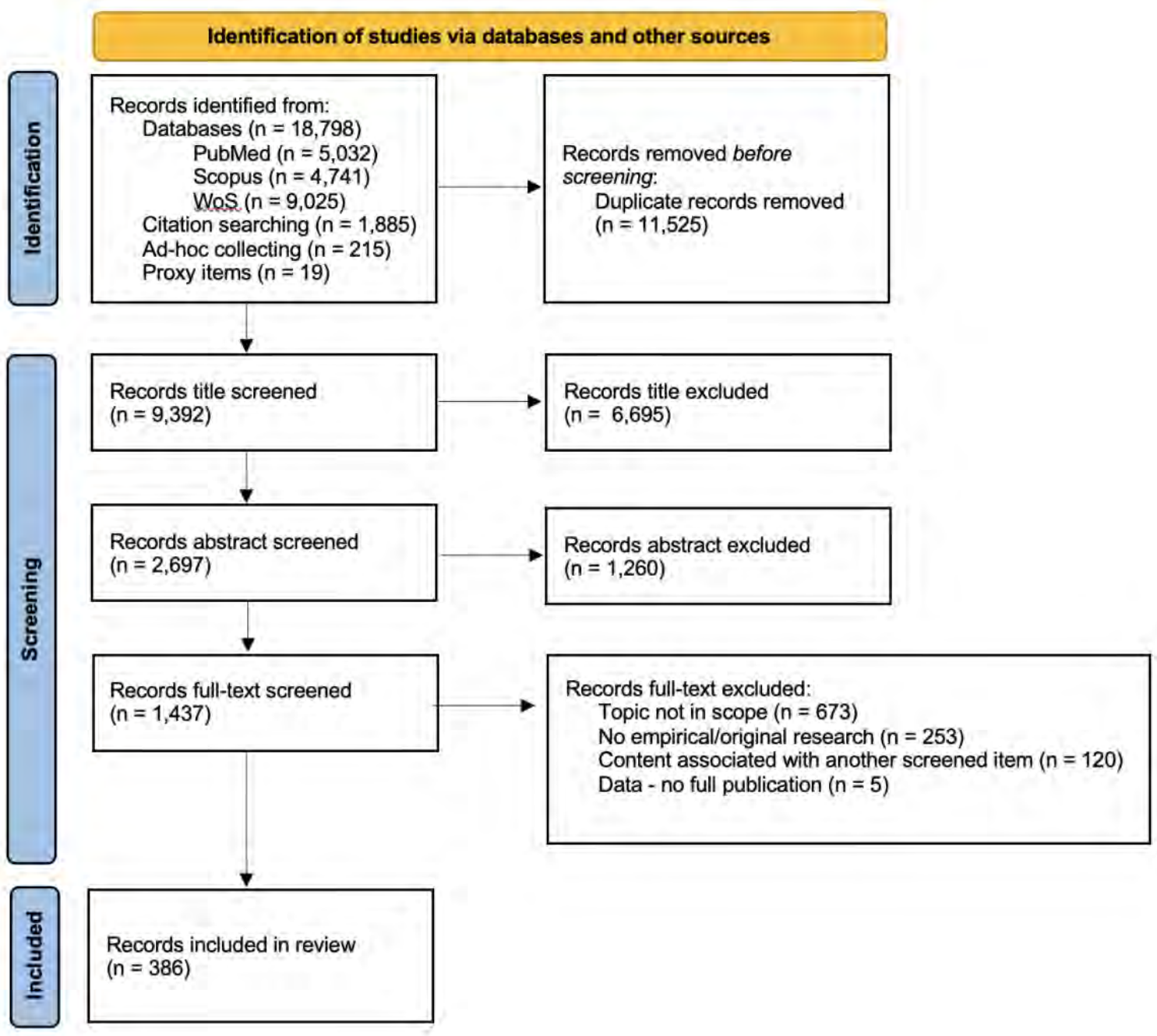

${ }^{1}$ Based on PRISMA 2020 flow diagram template (Page et al., 2021) 


\section{Version 1 of the Scoping Review of Empirical Research: List of 162 Central Items}

Ajiferuke, I., \& Adekannbi, J. O. (2018). Correction and retraction practices in library and information science journals. Journal of Librarianship and Information Science, In Press. https://doi.org/10.1177/0961000618785408

Alfaro-Toloza, P., Mayta-Tristan, P., \& Rodriguez-Morales, A. J. (2013). Publication misconduct and plagiarism retractions: A Latin American perspective. Current Medical Research and Opinion, 29(2), 99-100. https://doi.org/10.1185/03007995.2012.755504

Al-Ghareeb, A., Hillel, S., McKenna, L., Cleary, M., Visentin, D., Jones, M., Bressington, D., \& Gray, R. (2018). Retraction of publications in nursing and midwifery research: A systematic review. International Journal of Nursing Studies, 81, 8-13. https://doi.org/10.1016/j.ijnurstu.2018.01.013

Al-Hidabi, M. D. A., \& Teh, P. L. (2019). Multiple publications: The main reason for the retraction of papers in computer science. In K. Arai, S. Kapoor, \& R. Bhatia (Eds.), Advances in Information and Communication Networks (pp. 511-526). Springer International Publishing. https://doi.org/10.1007/978-3-030-03402-3 35

Almeida, R. M. V. R., de Albuquerque Rocha, K., Catelani, F., Fontes-Pereira, A. J., \& Vasconcelos, S. M. R. (2016). Plagiarism allegations account for most retractions in major Latin American/Caribbean databases. Science and Engineering Ethics, 22(5), 1447-1456. https://doi.org/10.1007/s11948-015-9714-5

AlRyalat, S. A., Azzam, M., Massad, A., \& Alqatawneh, D. (2020). Retractions of research papers by authors from the Arab region (1998-2018). European Science Editing, 46, e51002. https://doi.org/10.3897/ese.2020.e51002

Amos, K. A. (2014). The ethics of scholarly publishing: Exploring differences in plagiarism and duplicate publication across nations. Journal of the Medical Library Association : JMLA, 102(2), 87-91. https://doi.org/10.3163/1536-5050.102.2.005

Aspura, M. K. Y. I., Noorhidawati, A., \& Abrizah, A. (2018). An analysis of Malaysian retracted papers: Misconduct or mistakes? Scientometrics, 115(3), 1315-1328. https://doi.org/10.1007/s11192-018-2720-z

Atlas, M. C. (2004). Retraction policies of high-impact biomedical journals. Journal of the Medical Library Association, 92(2), 242-250.

Ayodele, F. O., Yao, L., \& Haron, H. (2019). Promoting ethics and integrity in management academic research: Retraction initiative. Science and Engineering Ethics, 25(2), 357382. https://doi.org/10.1007/s11948-017-9941-z

Azoulay, P., Furman, J. L., Krieger, \& Murray, F. (2015). Retractions. Review of Economics and Statistics, 97(5), 1118-1136. https://doi.org/10.1162/REST a 00469

Balhara, Y. P. S. (2015). A study exploring attributes and nature of the retracted literature on mental disorders. Indian Journal of Medical Ethics.

https://doi.org/10.20529/IJME.2015.007 
Balhara, Y. P. S., \& Mishra, A. (2014). Compliance of retraction notices for retracted articles on mental disorders with COPE guidelines on retraction. Current Science, 107(5), 757760.

Bar-llan, J., \& Halevi, G. (2017). Post retraction citations in context: A case study. Scientometrics, 113(1), 547-565. https://doi.org/10.1007/s11192-017-2242-0

Bar-llan, J., \& Halevi, G. (2018). Temporal characteristics of retracted articles. Scientometrics, 116(3), 1771-1783. https://doi.org/10.1007/s11192-018-2802-y

Bilbrey, E., O’Dell, N., \& Creamer, J. (2014). A novel rubric for rating the quality of retraction notices. Publications, 2(1), 14-26. https://doi.org/10.3390/publications2010014

Bolboacă, S. D., Buhai, D.-V., Aluaș, M., \& Bulboacă, A. E. (2019). Post retraction citations among manuscripts reporting a radiology-imaging diagnostic method. PLOS ONE, 14(6), e0217918. https://doi.org/10.1371/journal.pone.0217918

Bornemann-Cimenti, H., Szilagyi, I. S., \& Sandner-Kiesling, A. (2016). Perpetuation of retracted publications using the example of the Scott S. Reuben case: Incidences, reasons and possible improvements. Science and Engineering Ethics, 22(4), 10631072. https://doi.org/10.1007/s11948-015-9680-y

Bozzo, A., Bali, K., Evaniew, N., \& Ghert, M. (2017). Retractions in cancer research: A systematic survey. Research Integrity and Peer Review, 2(1). https://doi.org/10.1186/s41073-017-0031-1

Budd, J. M., Coble, Z., \& Abritis, A. (2016). An investigation of retracted articles in the biomedical literature. Proceedings of the Association for Information Science and Technology, 53(1), 1-9. https://doi.org/10.1002/pra2.2016.14505301055

Budd, J. M., Coble, Z. C., \& Anderson, K. M. (2011). Retracted publications in biomedicine: Cause for concern. Association of College and Research Libraries Conference, 390395.

Budd, J. M., Sievert, M., \& Schultz, T. R. (1998). Phenomena of retraction: Reasons for retraction and citations to the publications. JAMA, 280(3), 296. https://doi.org/10.1001/jama.280.3.296

Budd, J. M., Sievert, M., Schultz, T., \& Scoville, C. (1999). Effects of article retraction on citation and practice in medicine. Bulletin of the Medical Library Association, 87(4), 437-443. https://www.ncbi.n/m.nih.gov/pmc/articles/PMC226618/

Campos-Varela, I., \& Ruano-Raviña, A. (2019). Misconduct as the main cause for retraction. A descriptive study of retracted publications and their authors. Gaceta Sanitaria, 33(4), 356-360. https://doi.org/10.1016/i.gaceta.2018.01.009

Campos-Varela, I., Villaverde-Castañeda, R., \& Ruano-Raviña, A. (2019). Retraction of publications: A study of biomedical journals retracting publications based on impact factor and journal category. Gaceta Sanitaria, In Press.

https://doi.org/10.1016/i.gaceta.2019.05.008

Carey, L. C., Seth, A., Woolley, M., \& Woolley, K. L. (2016). Avoiding high-risk authors: Should Corporate Integrity Agreements recommend searching the Retracted 
Publications database? Current Medical Research and Opinion, 32(sup1), S5-S6. https://doi.org/10.1185/03007995.2016.1152850

Casadevall, A., Steen, R. G., \& Fang, F. C. (2014). Sources of error in the retracted scientific literature. The FASEB Journal, 28(9), 3847-3855.

https://doi.org/10.1096/fj.14-256735

Cassão, B. D., Herbella, F. A. M., Schlottmann, F., \& Patti, M. G. (2018). Retracted articles in surgery journals. What are surgeons doing wrong? Surgery, 163(6), 1201-1206. https://doi.org/10.1016/j.surg.2018.01.015

Chambers, L. M., Michener, C. M., \& Falcone, T. (2019). Plagiarism and data falsification are the most common reasons for retracted publications in obstetrics and gynaecology. Bjog-an International Journal of Obstetrics and Gynaecology, 126(9), 1134-1140. https://doi.org/10.1111/1471-0528.15689

Chauvin, A., De Villelongue, C., Pateron, D., \& Yordanov, Y. (2019). A systematic review of retracted publications in emergency medicine. European Journal of Emergency Medicine, 26(1), 19-23. https://doi.org/10.1097/MEJ.0000000000000491

Chen, C., Hu, Z., Milbank, J., \& Schultz, T. (2013). A visual analytic study of retracted articles in scientific literature. Journal of the American Society for Information Science and Technology, 64(2), 234-253. https://doi.org/10.1002/asi.22755

Chen, W., Xing, Q.-R., Wang, H., \& Wang, T. (2018). Retracted publications in the biomedical literature with authors from mainland China. Scientometrics, 114(1), 217227. https://doi.org/10.1007/s11192-017-2565-x

Cox, A., Craig, R., \& Tourish, D. (2018). Retraction statements and research malpractice in economics. Research Policy, 47(5), 924-935. https://doi.org/10.1016/i.respol.2018.02.016

Dal-Ré, R. (2019). [Analysis of biomedical Spanish articles retracted between 1970 and 2018] Análisis de los artículos españoles de biomedicina retractados entre 1970 y 2018. Medicina Clínica. https://doi.org/10.1016/j.medcli.2019.04.018

Dal-Ré, R., \& Ayuso, C. (2019). Reasons for and time to retraction of genetics articles published between 1970 and 2018. Journal of Medical Genetics, 56(11), 734-740. https://doi.org/10.1136/jmedgenet-2019-106137 (Correction published June 1, 2020, Journal of Medical Genetics, 57, p. 435-436.)

Damineni, R., Sardiwal, K., Waghle, S., \& Dakshyani, M. (2015). A comprehensive comparative analysis of articles retracted in 2012 and 2013 from the scholarly literature. Journal of International Society of Preventive and Community Dentistry, 5(1), 19. https://doi.org/10.4103/2231-0762.151968

Davis, P. M. (2012). The persistence of error: A study of retracted articles on the Internet and in personal libraries. Journal of the Medical Library Association: JMLA, 100(3), 184-189. https://doi.org/10.3163/1536-5050.100.3.008 (Correction published October 2012, Journal of the Medical Library Association : JMLA, 100(4), p. 329, https://doi.org/10.3163/1536-5050.100.4.027)

de Almeida, R. M. V. R., Catelani, F., Fontes-Pereira, A. J., \& de Souza Gave, N. (2015). Retractions in general and internal medicine in a high-profile scientific indexing 
database. Sao Paulo Medical Journal, 134(1), 74-78. https://doi.org/10.1590/15163180.2014 .00381601

Decullier, E., Huot, L., \& Maisonneuve, H. (2014). What time-lag for a retraction search on PubMed? BMC Research Notes, 7(1). https://doi.org/10.1186/1756-0500-7-395

Decullier, E., Huot, L., Samson, G., \& Maisonneuve, H. (2013). Visibility of retractions: A cross-sectional one-year study. BMC Research Notes, 6(1), 238.

https://doi.org/10.1186/1756-0500-6-238

Decullier, E., \& Maisonneuve, H. (2018). Correcting the literature: Improvement trends seen in contents of retraction notices. BMC Research Notes, 11(1), 490.

https://doi.org/10.1186/s13104-018-3576-2

Dinh, L., Sarol, J., Cheng, Y.-Y., Hsiao, T.-K., Parulian, N., \& Schneider, J. (2019). Systematic examination of pre- and post-retraction citations. Proceedings of the Association for Information Science and Technology, 56(1), 390-394. https://doi.org/10.1002/pra2.35

Duggar, D. C., Christopher, K. A., Tucker, B. E., Jones, D. A., Watson, M., Puckett, M., \& Wood, B. (1995). Promoting an awareness of retractions: The Louisiana State University Medical Center in Shreveport experience. Medical Reference Services Quarterly, 14(1), 17-32. https://doi.org/10.1300/J115V14N01 03

Elango, B., Kozak, M., \& Rajendran, P. (2019). Analysis of retractions in Indian science. Scientometrics, 119(2), 1081-1094. https://doi.org/10.1007/s11192-019-03079-y

Elia, N., Wager, E., \& Tramèr, M. R. (2014). Fate of articles that warranted retraction due to ethical concerns: A descriptive cross-sectional study. PLoS ONE, 9(1), e85846. https://doi.org/10.1371/journal.pone.0085846

El-Tahan, M. (2019). Can the similarity index predict the causes of retractions in highimpact anesthesia journals? A bibliometric analysis. Saudi Journal of Anaesthesia, 13(5), 2. https://doi.org/10.4103/sja.SJA 70918

Faggion Jr, C. M., Ware, R. S., Bakas, N., \& Wasiak, J. (2018). An analysis of retractions of dental publications. Journal of Dentistry, 79, 19-23.

https://doi.org/10.1016/j.jdent.2018.09.002

Fanelli, D. (2013). Why growing retractions are (mostly) a good sign. PLoS Medicine, 10(12), e1001563. https://doi.org/10.1371/journal.pmed.1001563

Fanelli, D., Costas, R., \& Larivière, V. (2015). Misconduct policies, academic culture and career stage, not gender or pressures to publish, affect scientific integrity. PLOS ONE, 10(6), e0127556. https://doi.org/10.1371/journal.pone.0127556

Fanelli, D., Ioannidis, J. P. A., \& Goodman, S. (2018). Improving the integrity of published science: An expanded taxonomy of retractions and corrections. European Journal of Clinical Investigation, 48(4), e12898. https://doi.org/10.1111/eci.12898

Fang, F. C., Steen, R. G., \& Casadevall, A. (2012). Misconduct accounts for the majority of retracted scientific publications. Proceedings of the National Academy of Sciences, 109(42), 17028-17033. https://doi.org/10.1073/pnas.1212247109 (Erratum published January 15, 2013, Proceedings of the National Academy of Sciences, 110(3), p. 1137, https://doi.org/10.1073/pnas.1220649110) 
Fang, Ferric C., \& Casadevall, A. (2011). Retracted science and the retraction index. Infection and Immunity, 79(10), 3855-3859. https://doi.org/10.1128/IAI.05661-11

Foo, J. Y. A. (2011). A retrospective analysis of the trend of retracted publications in the field of biomedical and life sciences. Science and Engineering Ethics, 17(3), 459-468. https://doi.org/10.1007/s11948-010-9212-8

Foo, J. Y. A., \& Tan, X. J. A. (2014). Analysis and implications of retraction period and coauthorship of fraudulent publications. Accountability in Research, 21(3), 198-210. https://doi.org/10.1080/08989621.2013.848799

Friedman, P. J. (1990). Correcting the literature following fraudulent publication. The Journal of the American Medical Association, 263(10), 1416-1419. https://doi.org/10.1001/jama.1990.03440100136019

Fulton, A. S., Coates, A. M., Williams, M. T., Howe, P. R. C., \& Hill, A. M. (2015). Persistent citation of the only published randomised controlled trial of Omega-3 supplementation in Chronic Obstructive Pulmonary Disease six years after Its retraction. Publications, 3(1), 7-26. https://doi.org/10.3390/publications3010017 (Correction published July 27, 2018, Publications, 6(3), p. 35, https://doi.org/10.3390/publications6030035)

Furman, J. L., Jensen, K., \& Murray, F. (2012). Governing knowledge in the scientific community: Exploring the role of retractions in biomedicine. Research Policy, 41(2), 276-290. https://doi.org/10.1016/j.respol.2011.11.001

Gasparyan, A. Y., Ayvazyan, L., Akazhanov, N. A., \& Kitas, G. D. (2014). Self-correction in biomedical publications and the scientific impact. Croatian Medical Journal, 55(1), 6172. https://doi.org/10.3325/cmj.2014.55.61

Greitemeyer, T. (2014). Article retracted, but the message lives on. Psychonomic Bulletin \& Review, 21(2), 557-561. https://doi.org/10.3758/s13423-013-0500-6

Greitemeyer, T., \& Sagioglou, C. (2015). Does exonerating an accused researcher restore the researcher's credibility? PLOS ONE, 10(5), e0126316.

https://doi.org/10.1371/journal.pone.0126316

Grieneisen, M. L., \& Zhang, M. (2012). A comprehensive survey of retracted articles from the scholarly literature. PLoS ONE, 7(10), e44118.

https://doi.org/10.1371/journal.pone.0044118

Hagberg, J. M. (2020). The unfortunately long life of some retracted biomedical research publications. Journal of Applied Physiology, japplphysiol.00003.2020. https://doi.org/10.1152/japplphysiol.00003.2020

Halevi, G. (2019). Why articles in arts and humanities are being retracted? Publishing Research Quarterly. https://doi.org/10.1007/s12109-019-09699-9

Hamilton, D. G. (2019). Continued citation of retracted radiation oncology literature-Do we have a problem? International Journal of Radiation Oncology $\bullet$ Biology $\bullet$ Physics, 103(5), 1036-1042. https://doi.org/10.1016/j.jirobp.2018.11.014

He, T. (2013). Retraction of global scientific publications from 2001 to 2010. Scientometrics, 96(2), 555-561. https://doi.org/10.1007/s11192-012-0906-3 
Hesselmann, F., \& Reinhart, M. (2019). Science means never having to say you're sorry? Apologies for scientific misconduct. Science Communication, 41(5), 552-579. https://doi.org/10.1177/1075547019860848

Hosseini, M., Hilhorst, M., de Beaufort, I., \& Fanelli, D. (2018). Doing the right thing: A qualitative investigation of retractions due to unintentional error. Science and Engineering Ethics, 24(1), 189-206. https://doi.org/10.1007/s11948-017-9894-2

Hu, G. (2017). Authorship of retraction notices: "If names are not rectified, then language will not be in accord with truth." Publications, 5(2), 10. https://doi.org/10.3390/publications5020010

Hughes, C. (1998). Academic medical libraries' policies and procedures for notifying library users of retracted scientific publications. Medical Reference Services Quarterly, 17(2), 37-42. https://doi.org/10.1300/J115v17n02 04

Huh, S., Kim, S. Y., \& Cho, H.-M. (2016). Characteristics of retractions from Korean Medical Journals in the KoreaMed database: A bibliometric analysis. PLOS ONE, 11(10), e0163588. https://doi.org/10.1371/journal.pone.0163588

Hwang, K., \& Wu, X. (2018). Retracted or withdrawn publications in journals relating to plastic surgery. Journal of Craniofacial Surgery, 29(5), 1114-1116.

https://doi.org/10.1097/SCS.0000000000004326

Inoue, Y., \& Muto, K. (2016). Noncompliance with human subjects' protection requirements as a reason for retracting papers: Survey of retraction notices on medical papers published from 1981 to 2011. Accountability in Research, 23(2), 123-135. https://doi.org/10.1080/08989621.2015.1069713

Jan, R., \& Zainab, T. (2018). The impact story of retracted articles: Altmetric it! 2018 5th International Symposium on Emerging Trends and Technologies in Libraries and Information Services (ETTLIS), 1-5. https://doi.org/10.1109/ETTLIS.2018.8485245

Jones, D. A., Watson, M. M., Comegys, M., Burnett, A., \& Tucker, B. (2003). Errata and retractions in electronic journals: Notification practices. Journal of Hospital Librarianship, 3(2), 19-27. https://doi.org/10.1300/J186v03n02 02

Karabag, S. F., \& Berggren, C. (2016). Misconduct, marginality and editorial practices in management, business and economics journals. PLOS ONE, 11(7), e0159492. https://doi.org/10.1371/journal.pone.0159492

Kim, S. Y., Yi, H. J., Cho, H.-M., \& Huh, S. (2019). How many retracted articles indexed in KoreaMed were cited 1 year after retraction notification. Science Editing, 6(2), 122127. https://doi.org/10.6087/kcse.172

King, E. G., Oransky, I., Sachs, T. E., Farber, A., Flynn, D. B., Abritis, A., Kalish, J. A., \& Siracuse, J. J. (2018). Analysis of retracted articles in the surgical literature. The American Journal of Surgery, 216(5), 851-855. https://doi.org/10.1016/j.amjsurg.2017.11.033

Kochan, C. A., \& Budd, J. M. (1992). The persistence of fraud in the literature: The Darsee case. Journal of the American Society for Information Science, 43(7), 488-493. https://doi.org/10.1002/(SICl)1097-4571(199208)43:7<488::AID-ASI3>3.0.CO;2-7 
Korpela, K. M. (2010). How long does it take for the scientific literature to purge itself of fraudulent material?: The Breuning case revisited. Current Medical Research and Opinion, 26(4), 843-847. https://doi.org/10.1185/03007991003603804

Kuroki, T., \& Ukawa, A. (2018). Repeating probability of authors with retracted scientific publications. Accountability in Research, 25(4), 212-219.

https://doi.org/10.1080/08989621.2018.1449651

Li, G., Kamel, M., Jin, Y., Xu, M., Mbuagbaw, L., Samaan, Z., Levine, M., \& Thabane, L. (2018). Exploring the characteristics, global distribution and reasons for retraction of published articles involving human research participants: A literature survey. Journal of Multidisciplinary Healthcare, Volume 11, 39-47. https://doi.org/10.2147/JMDH.S151745

Lu, S. F., Jin, G. Z., Uzzi, B., \& Jones, B. (2013). The retraction penalty: Evidence from the Web of Science. Scientific Reports, 3(1). https://doi.org/10.1038/srep03146

Madlock-Brown, C. R., \& Eichmann, D. (2015). The (lack of) impact of retraction on citation networks. Science and Engineering Ethics, 21(1), 127-137. https://doi.org/10.1007/s11948-014-9532-1

Marco-Cuenca, G., Salvador-Oliván, J.-A., \& Arquero-Avilés, R. (2019). [Ethics in biomedical scientific publication. A review of the publications retracted in Spain]Ética en la publicación científica biomédica. Revisión de las publicaciones retractadas en España. El Profesional de la Información, 28(2). https://doi.org/10.3145/epi.2019.mar.22

Markowitz, D. M., \& Hancock, J. T. (2016). Linguistic obfuscation in fraudulent science. Journal of Language and Social Psychology, 35(4), 435-445. https://doi.org/10.1177/0261927X15614605

McHugh, U. M., \& Yentis, S. M. (2019). An analysis of retractions of papers authored by Scott Reuben, Joachim Boldt and Yoshitaka Fujii. Anaesthesia, 74(1), 17-21. https://doi.org/10.1111/anae.14414 (Correction published December 4, 2018, Anaesthesia, 74(1), p. 113, https://doi.org/10.1111/anae.14515)

Mena, J. D., Ndoye, M., Cohen, A. J., Kamal, P., \& Breyer, B. N. (2019). The landscape of urological retractions: The prevalence of reported research misconduct. BJU International, 124(1), 174-179. https://doi.org/10.1111/bju.14706

Mine, S. (2019). Toward responsible scholarly communication and innovation: A survey of the prevalence of retracted articles on scholarly communication platforms. Proceedings of the Association for Information Science and Technology, 56(1), 738-739. https://doi.org/10.1002/pra2.155

Mistry, V., Grey, A., \& Bolland, M. J. (2019). Publication rates after the first retraction for biomedical researchers with multiple retracted publications. Accountability in Research, 26(5), 277-287. https://doi.org/10.1080/08989621.2019.1612244

Molckovsky, A., Vickers, M. M., \& Tang, P. A. (2011). Characterization of published errors in high-impact oncology journals. Current Oncology, 18(1). https://doi.org/10.3747/co.v18i1.707

Mongeon, P., \& Larivière, V. (2016). Costly collaborations: The impact of scientific fraud on co-authors' careers: Costly Collaborations: The Impact of Scientific Fraud on Co- 
Authors' Careers. Journal of the Association for Information Science and Technology, 67(3), 535-542. https://doi.org/10.1002/asi.23421

Mott, A., Fairhurst, C., \& Torgerson, D. (2019). Assessing the impact of retraction on the citation of randomized controlled trial reports: An interrupted time-series analysis. Journal of Health Services Research \& Policy, 24(1), 44-51. https://doi.org/10.1177/1355819618797965

Moylan, E. C., \& Kowalczuk, M. K. (2016). Why articles are retracted- a retrospective crosssectional study of retraction notices at BioMed Central. BMJ Open, 6(11), e012047. https://doi.org/10.1136/bmjopen-2016-012047

Nair, S., Yean, C., Yoo, J., Leff, J., Delphin, E., \& Adams, D. C. (2019). Reasons for article retraction in anesthesiology: A comprehensive analysis. Canadian Journal of Anesthesia/Journal Canadien d'anesthésie, Online first. https://doi.org/10.1007/s12630-019-01508-3

Nath, S. B., Marcus, S. C., \& Druss, B. G. (2006). Retractions in the research literature: Misconduct or mistakes? Medical Journal of Australia, 185(3), 152-154. https://doi.org/10.5694/j.1326-5377.2006.tb00504.x

Neale, A. V., Dailey, R. K., \& Abrams, J. (2010). Analysis of citations to biomedical articles affected by scientific misconduct. Science and Engineering Ethics, 16(2), 251-261. https://doi.org/10.1007/s11948-009-9151-4

Neale, A. V., Northrup, J., Dailey, R., Marks, E., \& Abrams, J. (2007). Correction and use of biomedical literature affected by scientific misconduct. Science and Engineering Ethics, 13, 5-24. https://doi.org/10.1007/s11948-006-0003-1

Nogueira, T. E., Gonçalves, A. S., Leles, C. R., Batista, A. C., \& Costa, L. R. (2017). A survey of retracted articles in dentistry. BMC Research Notes, 10(1). https://doi.org/10.1186/s13104-017-2576-y

Palla, I. A., Singson, M., \& Thiyagarajan, S. (2020). A comparative analysis of retracted papers in Health Sciences from China and India. Accountability in Research, 1-16. https://doi.org/10.1080/08989621.2020.1754804

Pantziarka, P., \& Meheus, L. (2019). Journal retractions in oncology: A bibliometric study. Future Oncology, 15(31), 3597-3608. https://doi.org/10.2217/fon-2019-0233

Parrish, D. M. (1999). Scientific misconduct and correcting the scientific literature: Academic Medicine, 74(3), 221-230. https://doi.org/10.1097/00001888-199903000$\underline{00009}$

Peterson, G. M. (2013). Characteristics of retracted open access biomedical literature: A bibliographic analysis. Journal of the American Society for Information Science and Technology, 64(12), 2428-2436. https://doi.org/10.1002/asi.22944

Pfeifer, M. P. (1992). Medical school libraries' handling of articles that report invalid science. Academic Medicine : Journal of the Association of American Medical Colleges, 67(2), 109-113. https://doi.org/10.1097/00001888-199202000-00014

Pfeifer, M. P., \& Snodgrass, G. L. (1990). The continued use of retracted, invalid scientific literature. The Journal of the American Medical Association, 263(10), 1420-1423. https://doi.org/10.1001/jama.1990.03440100140020 
Qi, X., Deng, H., \& Guo, X. (2017). Characteristics of retractions related to faked peer reviews: An overview. Postgraduate Medical Journal, 93(1102), 499-503. https://doi.org/10.1136/postgradmedj-2016-133969

Rabow, H., \& Rabow, I. (1999). Retraction of articles: Peer-review and quality control. In Libraries without limits: Changing needs_Changing roles (pp. 21-22).

Rada, R. (2005). A case study of a retracted systematic review on interactive health communication applications: Impact on media, scientists, and patients. Journal of Medical Internet Research, 7(2), e18. https://doi.org/10.2196/jmir.7.2.e18

Rada, R. F. (2007). Retractions, press releases and newspaper coverage. Health Information and Libraries Journal, 24(3), 210-215. https://doi.org/10.1111/j.1471$\underline{1842.2007 .00724 . x}$

Rai, R., \& Sabharwal, S. (2017). Retracted publications in orthopaedics: Prevalence, characteristics, and trends. The Journal of Bone and Joint Surgery, 99(9), e44. https://doi.org/10.2106/JBJS.16.01116

Rapani, A., Lombardi, T., Berton, F., Lupo, V. D., Lenarda, R. D., \& Stacchi, C. (n.d.). Retracted publications and their citation in dental literature: A systematic review. Clinical and Experimental Dental Research, n/a(n/a). https://doi.org/10.1002/cre2.292

Redman, B. K., Yarandi, H. N., \& Merz, J. F. (2008). Empirical developments in retraction. Journal of Medical Ethics, 34(11), 807-809. https://doi.org/10.1136/jme.2007.023069

Resnik, D. B., \& Dinse, G. E. (2013). Scientific retractions and corrections related to misconduct findings. Journal of Medical Ethics, 39(1), 46-50. https://doi.org/10.1136/medethics-2012-100766

Resnik, D. B., Wager, E., \& Kissling, G. E. (2015). Retraction policies of top scientific journals ranked by impact factor. Journal of the Medical Library Association: JMLA, 103(3), 136-139. https://doi.org/10.3163/1536-5050.103.3.006

Ribeiro, M. D., \& Vasconcelos, S. M. R. (2018). Retractions covered by Retraction Watch in the 2013-2015 period: Prevalence for the most productive countries. Scientometrics, 114(2), 719-734. https://doi.org/10.1007/s11192-017-2621-6 (Correction published January 31, 2018, Scientometrics, 114, p. 735, https://doi.org/10.1007/s11192-0182653-6)

Roe, P., \& Lewison, G. (2019). The anatomy of retracted papers in the Web of Science, 1998-2017. In G. Catalano, C. Daraio, M. Gregori, H. F. Moed, \& G. Ruocco (Eds.), Proceedings of the 17th International Conference on Scientometrics and Informetrics, ISSI 2019, Rome, Italy, September 2-5, 2019 (pp. 59-64). ISSI Society.

Rosenkrantz, A. B. (2016). Retracted publications within radiology journals. American Journal of Roentgenology, 206(2), 231-235. https://doi.org/10.2214/AJR.15.15163 (Correction published April 2016, American Journal of Roentgenology, 206(4), p. 901, https://doi.org/10.2214/AJR.16.16250)

Rubbo, P., Helmann, C. L., Bilynkievycz dos Santos, C., \& Pilatti, L. A. (2019). Retractions in the engineering field: A study on the Web of Science database. Ethics \& Behavior, 29(2), 141-155. https://doi.org/10.1080/10508422.2017.1390667 
Rubbo, P., Pilatti, L. A., \& Picinin, C. T. (2019). Citation of retracted articles in engineering: A study of the Web of Science database. Ethics \& Behavior, 29(8), 661-679. https://doi.org/10.1080/10508422.2018.1559064

Saikia, P., \& Thakuria, B. (2019). Retraction of papers authored by Yuhji Saitoh - Beyond the Fujii phenomenon. Indian Journal of Anaesthesia, 63(7), 571-584. https://doi.org/10.4103/ija.IJA 26719

Samp, J. C., Schumock, G. T., \& Pickard, A. S. (2012). Retracted publications in the drug literature. Pharmacotherapy: The Journal of Human Pharmacology and Drug Therapy, 32(7), 586-595. https://doi.org/10.1002/j.1875-9114.2012.01100.x

Schmidt, M. (2018). An analysis of the validity of retraction annotation in PubMed and the Web of Science. Journal of the Association for Information Science and Technology, 69(2), 318-328. https://doi.org/10.1002/asi.23913

Shamim, T. (2018). Data regarding articles retracted from PubMed indexed dental journals from India. Data in Brief, 18, 1069-1072. https://doi.org/10.1016/i.dib.2018.03.133

Shema, H., Hahn, O., Mazarakis, A., \& Peters, I. (2019). Retractions from altmetric and bibliometric perspectives. Information - Wissenschaft \& Praxis, 70(2-3), 98-110. https://doi.org/10.1515/iwp-2019-2006

Shuai, X., Rollins, J., Moulinier, I., Custis, T., Edmunds, M., \& Schilder, F. (2017). A multidimensional investigation of the effects of publication retraction on scholarly impact. Journal of the Association for Information Science and Technology, 68(9), 2225-2236. https://doi.org/10.1002/asi.23826

Singh, H. P., Mahendra, A., Yadav, B., Singh, H., Arora, N., \& Arora, M. (2014). A comprehensive analysis of articles retracted between 2004 and 2013 from biomedical literature - A call for reforms. Journal of Traditional and Complementary Medicine, 4(3), 136-139. https://doi.org/10.4103/2225-4110.136264

Snodgrass, G. L., \& Pfeifer, M. P. (1992). The characteristics of medical retraction notices. Bulletin of the Medical Library Association, 80(4), 328-334.

Souder, L. (2010). A rhetorical analysis of apologies for scientific misconduct: Do they really mean it? Science and Engineering Ethics, 16(1), 175-184. https://doi.org/10.1007/s11948-009-9149-y

Stavale, R., Ferreira, G. I., Galvão, J. A. M., Zicker, F., Novaes, M. R. C. G., Oliveira, C. M. de, \& Guilhem, D. (2019). Research misconduct in health and life sciences research: A systematic review of retracted literature from Brazilian institutions. PLOS ONE, 14(4), e0214272. https://doi.org/10.1371/journal.pone.0214272

Steen, R. G. (2011a). Retractions in the medical literature: Who is responsible for scientific integrity? American Medical Writers Association Journal, 26(1), 2-7.

Steen, R. G. (2011b). Retractions in the scientific literature: Is the incidence of research fraud increasing? Journal of Medical Ethics, 37(4), 249-253.

Steen, R. G. (2011c). Retractions in the scientific literature: Do authors deliberately commit research fraud? Journal of Medical Ethics, 37(2), 113-117.

https://doi.org/10.1136/jme.2010.038125 
Steen, R. G. (2011d). Retractions in the medical literature: How many patients are put at risk by flawed research? Journal of Medical Ethics, 37(11), 688-692.

https://doi.org/10.1136/jme.2011.043133

Steen, R. G. (2012). Retractions in the medical literature: How can patients be protected from risk? Journal of Medical Ethics, 38(4), 228-232.

Steen, R. G., Casadevall, A., \& Fang, F. C. (2013). Why has the number of scientific retractions increased? PLOS ONE, 8(7), 9.

https://doi.org/10.1371/journal.pone.0068397 (Correction published July 19, 2013, PLOS ONE, 8(7), https://doi.org/10.1371/annotation/0d28db18-e117-4804-b1bce2da285103ac)

Stern, A. M., Casadevall, A., Steen, R. G., \& Fang, F. C. (2014). Financial costs and personal consequences of research misconduct resulting in retracted publications. ELife, 3. https://doi.org/10.7554/eLife.02956

Stretton, S., Bramich, N. J., Keys, J. R., Monk, J. A., Ely, J. A., Haley, C., Woolley, M. J., \& Woolley, K. L. (2012). Publication misconduct and plagiarism retractions: A systematic, retrospective study. Current Medical Research and Opinion, 28(10), 1575-1583. https://doi.org/10.1185/03007995.2012.728131

Suelzer, E. M., Deal, J., Hanus, K. L., Ruggeri, B., Sieracki, R., \& Witkowski, E. (2019). Assessment of citations of the retracted article by Wakefield et al with fraudulent claims of an association between vaccination and autism. JAMA Network Open, 2(11), e1915552. https://doi.org/10.1001/jamanetworkopen.2019.15552

Teixeira da Silva, J. A., \& Dobránszki, J. (2017). Highly cited retracted papers. Scientometrics, 110(3), 1653-1661. https://doi.org/10.1007/s11192-016-2227-4

Torres, K. O., \& Michel, M. T. (2018). Retratação de artigos médicos e suas implicações éticas. Revista Latinoamericana de Bioética, 18(2), 100-125. https://dx.doi.org/10.18359/rlbi.3446

Tourish, D., \& Craig, R. (2020). Research misconduct in business and management studies: Causes, consequences, and possible remedies. Journal of Management Inquiry, 29(2), 174-187. https://doi.org/10.1177/1056492618792621

Trikalinos, N. A., Evangelou, E., \& loannidis, J. P. A. (2008). Falsified papers in high-impact journals were slow to retract and indistinguishable from nonfraudulent papers. Journal of Clinical Epidemiology, 61(5), 464-470. https://doi.org/10.1016/j.jclinepi.2007.11.019

Tripathi, M., Dwivedi, G., Sonkar, S. K., \& Kumar, S. (2018). Analysing retraction notices of scholarly journals: A study. DESIDOC Journal of Library \& Information Technology, 38(5), 305. https://doi.org/10.14429/djlit.38.5.13103

Tripathi, M., Sonkar, S. K., \& Kumar, S. (2019). A cross sectional study of retraction notices of scholarly journals of science. DESIDOC Journal of Library \& Information Technology, 39(2), 74-81. https://doi.org/10.14429/djlit.39.2.14000

van der Vet, P. E., \& Nijveen, H. (2016). Propagation of errors in citation networks: A study involving the entire citation network of a widely cited paper published in, and later retracted from, the journal Nature. Research Integrity and Peer Review, 1(1). https://doi.org/10.1186/s41073-016-0008-5 
Vuong, Q.-H. (2020). The limitations of retraction notices and the heroic acts of authors who correct the scholarly record: An analysis of retractions of papers published from 1975 to 2019. Learned Publishing. https://doi.org/10.1002/leap.1282

Vuong, Q.-H., La, V.-P., Ho, M.-T., Vuong, T.-T., \& Ho, M.-T. (2020). Characteristics of retracted articles based on retraction data from online sources through February 2019. Science Editing, 7(1), 34-44. https://doi.org/10.6087/kcse.187

Wager, E., \& Williams, P. (2011). Why and how do journals retract articles? An analysis of Medline retractions 1988-2008. Journal of Medical Ethics, 37(9), 567-570. https://doi.org/10.1136/ime.2010.040964

Wang, J., Ku, J. C., Alotaibi, N. M., \& Rutka, J. T. (2017). Retraction of neurosurgical publications: A systematic review. World Neurosurgery, 103, 809-814.e1. https://doi.org/10.1016/j.wneu.2017.04.014

Wang, T., Xing, Q.-R., Wang, H., \& Chen, W. (2019). Retracted publications in the biomedical literature from open access journals. Science and Engineering Ethics, 25(3), 855-868. https://doi.org/10.1007/s11948-018-0040-6

Wasiak, J., George Hamilton, D., Foroudi, F., \& Faggion, C. M. (2018). Surveying retracted studies and notices within the field of radiation oncology. International Journal of Radiation Oncology, Biology, Physics, 102(3), 660-665. https://doi.org/10.1016/j.ijrobp.2018.06.028

Whitely, W. P. (1994). The scientific community's response to evidence of fraudulent publication. The Robert Slutsky case. JAMA: The Journal of the American Medical Association, 272(2), 170-173. https://doi.org/10.1001/jama.272.2.170

Wiedermann, C. J. (2018). Inaction over retractions of identified fraudulent publications: Ongoing weakness in the system of scientific self-correction. Accountability in Research, 25(4), 239-253. https://doi.org/10.1080/08989621.2018.1450143

Williams, P., \& Wager, E. (2013). Exploring why and how journal editors retract articles: Findings from a qualitative study. Science and Engineering Ethics, 19(1), 1-11. https://doi.org/10.1007/s11948-011-9292-0

Woolley, K. L., Lew, R. A., Stretton, S., Ely, J. A., Bramich, N. J., Keys, J. R., Monk, J. A., \& Woolley, M. J. (2011). Lack of involvement of medical writers and the pharmaceutical industry in publications retracted for misconduct: A systematic, controlled, retrospective study. Current Medical Research and Opinion, 27(6), 1175-1182. https://doi.org/10.1185/03007995.2011.573546

Woolley, K., Woolley, M., Lew, R., Bramich, M., Ely, J., Stretton, S., Monk, J., \& Keys, J. (2010). Round up the usual suspects? Involvement of medical writers and the pharmaceutical industry in retracted publications. Current Medical Research and Opinion, 26, S11-S11.

Wray, K. B., \& Andersen, L. E. (2018). Retractions in Science. Scientometrics, 117(3), 2009-2019. https://doi.org/10.1007/s11192-018-2922-4

Wright, K., \& McDaid, C. (2011). Reporting of article retractions in bibliographic databases and online journals. Journal of the Medical Library Association: JMLA, 99(2), 164-167. https://doi.org/10.3163/1536-5050.99.2.010 
Wright, N. (1991). A Citation Context Analysis of Retracted Scientific Articles. University of Maryland at College Park.

Xu, S., \& Hu, G. (2018). Retraction notices: Who authored them? Publications, 6(1), 2. https://doi.org/10.3390/publications6010002

Yan, J., MacDonald, A., Baisi, L.-P., Evaniew, N., Bhandari, M., \& Ghert, M. (2016). Retractions in orthopaedic research: A systematic review. Bone \& Joint Research, 5(6), 263-268. https://doi.org/10.1302/2046-3758.56.BJR-2016-0047

Yuan, J., Feng, L., \& Yang, L. (2019). A multi-dimensional observation framework of retracted publications. In G. Catalano, C. Daraio, M. Gregori, H. F. Moed, \& G. Ruocco (Eds.), Proceedings of the 17th International Conference on Scientometrics and Informetrics, ISSI 2019, Rome, Italy, September 2-5, 2019 (pp. 1358-1367). ISSI Society.

Zhang, M., \& Grieneisen, M. L. (2013). The impact of misconduct on the published medical and non-medical literature, and the news media. Scientometrics, 96(2), 573-587. https://doi.org/10.1007/s11192-012-0920-5 


\section{Version 2.15 of the Scoping Review of Empirical Research: List of 386 Central Items}

This list is also available in an interactive dynamic bibliography at https://infoqualitylab.org/projects/risrs2020/bibliography/

Abbas, M., Pires, D., Peters, A., Morel, C. M., Hurst, S., Holmes, A., Saito, H., Allegranzi, B., Lucet, J. C., Zingg, W., Harbarth, S., \& Pittet, D. (2018). Conflicts of interest in infection prevention and control research: No smoke without fire. A narrative review. Intensive Care Medicine, 44(10), 1679-1690. https://doi.org/10.1007/s00134-018$\underline{5361-z}$

Abou-Setta, A. M., Rabbani, R., Lix, L. M., Turgeon, A. F., Houston, B. L., Fergusson, D. A., \& Zarychanski, R. (2019). Can authorship bias be detected in meta-analysis? Canadian Journal of Anesthesia/Journal Canadien d'Anesthésie, 66(3), 287-292. https://doi.org/10.1007/s12630-018-01268-6

Abritis, A. J. (2015). An Assessment of Retractions as a Measure of Scientific Misconduct and Impact on Public Health Risks [Doctoral dissertation, University of South Florida]. https://scholarcommons.usf.edu/etd/5630/

Achsan, H. T. Y., Suhartanto, H., \& Wibowo, W. C. (2019). A technique in information retrieval and bibliometrics to check the reliability of an article indexing. International Conference on Contemporary Computing and Informatics, 148-153. https://doi.org/10.1109/IC3I46837.2019.9055651

Agarwal, A., \& loannidis, J. P. A. (2019). PREDIMED trial of Mediterranean diet: Retracted, republished, still trusted? BMJ, 364(8187), I341. https://doi.org/10.1136/bmj.|I341

Ajiferuke, I., \& Adekannbi, J. O. (2020). Correction and retraction practices in library and information science journals. Journal of Librarianship and Information Science, 52(1), 169-183. https://doi.org/10.1177/0961000618785408

Akhaddar, A. (2021). Error publication (published erratum) in neurosurgical journals worldwide using PubMed during the last 30 years. Child's Nervous System, 37, 637643. https://doi.org/10.1007/s00381-020-04824-y

Alfaro-Toloza, P., Mayta-Tristan, P., \& Rodriguez-Morales, A. J. (2013). Publication misconduct and plagiarism retractions: A Latin American perspective. Current Medical Research and Opinion, 29(2), 99-100. https://doi.org/10.1185/03007995.2012.755504

Al-Ghareeb, A., Hillel, S., McKenna, L., Cleary, M., Visentin, D., Jones, M., Bressington, D., \& Gray, R. (2018). Retraction of publications in nursing and midwifery research: $A$ systematic review. International Journal of Nursing Studies, 81, 8-13. https://doi.org/10.1016/j.jinurstu.2018.01.013

Al-Hidabi, M. D. A., \& Teh, P. L. (2018). Multiple publications: The main reason for the retraction of papers in computer science. Future of Information and Communication Conference, 886, 511-526. https://doi.org/10.1007/978-3-030-03402-3 35

Almeida Renan Moritz, V. R., de Albuquerque Rocha, K., Catelani, F., Fontes-Pereira, A. J., \& Vasconcelos, S. M. R. (2016). Plagiarism allegations account for most retractions in major Latin American/Caribbean databases. Science and Engineering Ethics, 22(5), 1447-1456. https://doi.org/10.1007/s11948-015-9714-5 
Alrawadieh, Z., \& Zareer, A. (2019). Exploring retraction in tourism and hospitality journals. European Journal of Tourism Research, 22, 20-30.

https://ejtr.vumk.eu/index.php/about/article/view/372

Alryalat, S. A., Azzam, M., Massad, A., \& Alqatawneh, D. (2020). Retractions of research papers by authors from the Arab region (1998-2018). European Science Editing, 46, 14. https://doi.org/10.3897/ese.2020.e51002

Alvaro, E. (2013). Retractions in chemistry: Prevalence and impact. American Chemical Society National Meeting, Indianapolis, IN.

https://www.scholars.northwestern.edu/en/publications/retractions-in-chemistryprevalence-and-impact

Alvaro, E. (2012). Impact of retractions on the chemical literature. American Chemical Society National Meeting, San Diego, CA.

https://www.scholars.northwestern.edu/en/publications/impact-of-retractions-on-thechemical-literature

Amos, K. A. (2014). The ethics of scholarly publishing: Exploring differences in plagiarism and duplicate publication across nations. Journal of the Medical Library Association, 102(2), 87-91. https://doi.org/10.3163/1536-5050.102.2.005

Ana, J., Koehlmoos, T., Smith, R., \& Yan Lijing, L. (2013). Research misconduct in low- and middle-income countries. PLoS Medicine, 10(3), e1001315.

https://doi.org/10.1371/journal.pmed.1001315

Andersen, L. E., \& Wray, K. B. (2019). Detecting errors that result in retractions. Social Studies of Science, 49(6), 942-954. https://doi.org/10.1177/0306312719872008

Andersen, N., Corr, P. J., \& Furnham, A. (2021). A bibliometric analysis of H. J. Eysenck's research output: Clarifying controversy. Personality and Individual Differences, 169, 109935. https://doi.org/10.1016/j.paid.2020.109935

Aspura, M. K. Y. I., Noorhidawati, A., \& Abrizah, A. (2018). An analysis of Malaysian retracted papers: Misconduct or mistakes? Scientometrics, 115(3), 1315-1328. https://doi.org/10.1007/s11192-018-2720-z

Ataie-Ashtiani, B. (2018). World map of scientific misconduct. Science and Engineering Ethics, 24(5), 1653-1656. https://doi.org/10.1007/s11948-017-9939-6 (Erratum published July 18, 2017, Science and Engineering Ethics, 24, p. 1657, https://doi.org/10.1007/s11948-017-9942-y)

Atlas, M. C. (2004). Retraction policies of high-impact biomedical journals. Journal of the Medical Library Association, 92(2), 242-250. https://www.ncbi.nlm.nih.gov/pmc/articles/PMC385306/

Avenell, A., Stewart, F., Grey, A., Gamble, G., \& Bolland, M. (2019). An investigation into the impact and implications of published papers from retracted research: Systematic search of affected literature. BMJ Open, 9, e031909. https://doi.org/10.1136/bmjopen2019-031909 (Correction published December 1, 2019, BMJ Open, 9, p. e031909, https://doi.org/10.1136/bmjopen-2019-031909corr1)

Ayodele, F. O., Yao, L., \& Haron, H. (2019). Promoting ethics and integrity in management academic research: Retraction initiative. Science and Engineering Ethics, 25(2), 357382. https://doi.org/10.1007/s11948-017-9941-z 
Azoulay, P., Furman, J. L., Krieger, J. L., \& Murray, F. (2015). Retractions. Review of Economics and Statistics, 97(5), 1118-1136. https://doi.org/10.1162/REST a 00469

Azoulay, P., Bonatti, A., \& Krieger, J. L. (2017). The career effects of scandal: Evidence from scientific retractions. Research Policy, 46(9), 1552-1569. https://doi.org/10.1016/j.respol.2017.07.003

Badreldin, H., Ghawaa, Y., Alshehri, A., Alzaharani, M., \& Almubarak, A. (2020). Exploratory analysis of retracted cardiovascular diseases publications. Research Square. https://doi.org/10.21203/rs.2.22359/v1

Bakker, C. J., Brown, S. J., \& Theis-Mahon, N. (2021). Are there retractions in your systematic review? Findings from the pharmaceutical literature. Medical Library Association Annual Meeting.

Bakker, C. J., \& Riegelman, A. (2018). Retracted publications in mental health literature: Discovery across bibliographic platforms. Journal of Librarianship and Scholarly Communication, 6(1), eP2199. https://doi.org/10.7710/2162-3309.2199

Balhara, Y. P. S., \& Mishra, A. (2014). Compliance of retraction notices for retracted articles on mental disorders with COPE guidelines on retraction. Current Science, 107(5), 757760. https://wwwops.currentscience.ac.in/Volumes/107/05/0757.pdf

Balhara, Y. P. S., \& Mishra, A. (2015). A study exploring attributes and nature of the retracted literature on mental disorders. Indian Journal of Medical Ethics, 12(1), 30. https://doi.org/10.20529/IJME.2015.007

Bar-llan, J., \& Halevi, G. (2017). Post retraction citations in context: A case study. Scientometrics, 113(1), 547-565. https://doi.org/10.1007/s11192-017-2242-0

Bar-Ilan, J., \& Halevi, G. (2018). Temporal characteristics of retracted articles. Scientometrics, 116(3), 1771-1783. https://doi.org/10.1007/s11192-018-2802-y

Bar-Ilan, J., \& Halevi, G. (2020). Retracted articles-the scientific version of fake news. In Greifeneder Rainer, Jaffé Mariela E, Newman Eryn J, \& Schwarz Norbert (Eds.), The Psychology of Fake News: Accepting, Sharing, and Correcting Misinformation (pp. 4770). Routledge.

https://dornsife.usc.edu/assets/sites/832/docs/Schwarz Jalbert2020.pdf\#page=60

Bar-llan, J., \& Halevi, G. (2019). Retracted research articles from the RetractionWatch data base: Research in progress paper. International Conference on Scientometrics \& Informetrics, Vol. I, 322-328. https://www.issisociety.org/proceedings/issi 2019/ISSI\%202019\%20\%20Proceedings $\% 20$ VOLUME\%20I.pdf

Barnett, B. S., \& Doblin, R. (2021). Dissemination of erroneous research findings and subsequent retraction in high-circulation newspapers: A case study of alleged MDMAinduced dopaminergic neurotoxicity in primates. Journal of Psychoactive Drugs, 53(2), 104-110. https://doi.org/10.1080/02791072.2020.1847365

Baron, J. N., King, M. D., \& Sorenson, O. (2016). She blinded me with science: The sociology of scientific misconduct. In Palmer D, Smith-Crowe K, \& Greenwood R (Eds.), Organizational Wrongdoing: Key Perspectives and New Directions (pp. 176202). Cambridge University Press. https://doi.org/10.1017/CBO9781316338827.008 
Benchimol, E. I., Moher, D., Ehrenstein, V., \& Langan, S.M. (2020). Retraction of COVID-19 pharmacoepidemiology research could have been avoided by effective use of reporting guidelines. Clinical Epidemiology, 12, 1403-1420.

https://doi.org/10.2147/CLEP.S288677

Bendezu, Q. G. J. P., Hernández-Vásquez, A., \& Comandé, D. (2020). Retracted publications about COVID-19 in scientific journals: A living systematic review. Open Science Framework. https://doi.org/10.17605/OSF.IO/JK3XG

Bennett, C., Chambers, L. M., Al-Hafez, L., Michener, C. M., Falcone, T., Yao, M., \& Berghella, V. (2020). Retracted articles in the obstetrics literature: Lessons from the past to change the future. American Journal of Obstetrics \& Gynecology MFM, 2(4), 100201. https://doi.org/10.1016/j.ajogmf.2020.100201

Ben-Yehuda, N., \& Oliver-Lumerman, A. (2017a). An organizational approach to research fraud. In Fraud and Misconduct in Research (pp. 63-92). University of Michigan Press. http://www.jstor.org/stable/10.3998/mpub.9717920.6

Ben-Yehuda, N. \& Oliver-Lumerman, A. (2017b). Fraud in research: Frequency patterns. In Fraud and Misconduct in Research (pp. 19-62). University of Michigan Press. http://www.jstor.org/stable/10.3998/mpub.9717920.5

Berggren, C., \& Karabag, S. F. (2019). Scientific misconduct at an elite medical institute: The role of competing institutional logics and fragmented control. Research Policy, 48(2), 428-443. https://doi.org/10.1016/j.respol.2018.03.020

Berland, N., Piot, C., \& Capkun, V. (2015). Éditorial: Le retrait d'articles en sciences comptables. Comptabilite Controle Audit, 21(2), 5-12. https://doi.org/10.3917/cca.212.0005

Bhargava, M., Vaswani, V., \& Vaswani, R. (2020). Ethics-related guidelines for authors and article retractions: How do Indian biomedical journals measure up? Indian Journal of Medical Ethics, V(1), 25-33. https://doi.org/10.20529/IJME.2019.076

Bik, E. M., Fang, F. C., Kullas, A. L., Davis, R. J., \& Casadevall, A. (2018). Analysis and correction of inappropriate image duplication: The Molecular and Cellular Biology experience. Molecular and Cellular Biology, 38(20), e00309-18. https://doi.org/10.1128/MCB.00309-18

Bilbrey, E., O'Dell, N., \& Creamer, J. (2014). A novel rubric for rating the quality of retraction notices. Publications, 2(1), 14-26. https://doi.org/10.3390/publications2010014

Bolboacă, S. D, Buhai, D. V., Aluaș, M., \& Bolboacă, A. E. (2019). Post retraction citations among manuscripts reporting a radiology-imaging diagnostic method. PLOS ONE, 14(6), e0217918. https://doi.org/10.1371/journal.pone.0217918

Bolland, M. J., Avenell, A., Gamble, G. D., Buranyi, S., \& Grey, A. (2018). A randomised investigation of journal responses to academic and journalist enquiry about possible scientific misconduct. BMC Research Notes, 11(1), 521. https://doi.org/10.1186/s13104-018-3613-1

Bolland, M. J., Grey, A., Avenell, A., \& Klein, A. A. (2021). Correcting the scientific record-A broken system? Accountability in Research, 28(5), 265-279.

https://doi.org/10.1080/08989621.2020.1852938 
Bolland, M. J., Grey, A., \& Avenell, A. (2021). Citation of retracted publications: A challenging problem [Online First]. Accountability in Research.

https://doi.org/10.1080/08989621.2021.1886933

Bordignon, F. (2020). Self-correction of science: A comparative study of negative citations and post-publication peer review. Scientometrics, 124(2), 1225-1239.

https://doi.org/10.1007/s11192-020-03536-z

Bordino, M., Ravizzotti, E., \& Vercelli, S. (2020). Retracted articles in rehabilitation: Just the tip of the iceberg? A bibliometric analysis. Archives of Physiotherapy, 10(1), 21. https://doi.org/10.1186/s40945-020-00092-w

Bornemann-Cimenti, H., Szilagyi, I. S., \& Sandner-Kiesling, A. (2016). Perpetuation of retracted publications using the example of the Scott S. Reuben Case: Incidences, reasons and possible improvements. Science and Engineering Ethics, 22(4), 10631072. https://doi.org/10.1007/s11948-015-9680-y

Bornmann, L. \& Haunschild, R. (2018). Allegation of scientific misconduct increases Twitter attention. Scientometrics, 115(2), 1097-1100. https://doi.org/10.1007/s11192-018$\underline{2698-6}$

Bosch, X., Hernández, C., Pericas, J. M., Doti, P., \& Marušić, A. (2012). Misconduct policies in high-impact biomedical journals. PLoS ONE, 7(12), e51928.

https://doi.org/10.1371/journal.pone.0051928

Bozzo, A., Bali, K., Evaniew, N., \& Ghert, M. (2017). Retractions in cancer research: A systematic survey. Research Integrity and Peer Review, 2(1), 5. https://doi.org/10.1186/s41073-017-0031-1

Brainard, J., You, J., \& Bonazzi, D. (2018). Rethinking retractions: The largest-ever database of retracted articles suggests the burgeoning numbers reflect better oversight, not a crisis in science. Science, 362(6413), 390-393.

https://doi.org/10.1126/science.362.6413.390

Bramstedt, K. A. (2020). The carnage of substandard research during the COVID-19 pandemic: A call for quality. Journal of Medical Ethics, 46(12), 803-807. https://doi.org/10.1136/medethics-2020-106494

Brookes, P. S. (2014). Internet publicity of data problems in the bioscience literature correlates with enhanced corrective action. PeerJ, 2, e313.

https://doi.org/10.7717/peerj.313

Brown, S. J., Bakker, C. J., \& Theis-Mahon, N. (2020). Flawed research in focus: Retracted publications in pharmacy systematic reviews. Medical Library Association Annual Meeting. Medical Library Association Annual Meeting. https://experts.umn.edu/en/publications/flawed-research-in-focus-retractedpublications-in-pharmacy-syste

Bucci, E. M. (2018). Automatic detection of image manipulations in the biomedical literature article. Cell Death and Disease, 9(3), 400. https://doi.org/10.1038/s41419-018-0430-3

Budd, J. M., Coble, Z. C., \& Abritis, A. J. (2016). An investigation of retracted articles in the biomedical literature. Annual Meeting of the Association for Information Science and Technology, 53(1), 1-9. https://doi.org/10.1002/pra2.2016.14505301055 
Budd, J. M., Coble, Z. C., \& Anderson, K. M. (2011). Retracted publications in biomedicine: Cause for concern. Association of College and Research Libraries Conference, 390395.

http://www.ala.org/acrl/sites/ala.org.acrl/files/content/conferences/confsandpreconfs/na tional/2011/papers/retracted publicatio.pdf

Budd, J. M., Sievert, M., Schultz, T. R., \& Scoville, C. (1999). Effects of article retraction on citation and practice in medicine. Bulletin of the Medical Library Association, 87(4), 437-443. https://www.ncbi.nlm.nih.gov/pmc/articles/PMC226618/

Budd, J. M., Sievert, M., \& Schultz, T. R. (1998). Phenomena of retraction: Reasons for retraction and citations to the publications. JAMA, 280(3), 296-297.

https://doi.org/10.1001/jama.280.3.296

Bülow, W., Godskesen, T. E., Helgesson, G., \& Eriksson, S. (2020). Why unethical papers should be retracted [Online First]. Journal of Medical Ethics. https://doi.org/10.1136/medethics-2020-106140

Byrne, J. A., Park, Y., West, R. A., Capes-Davis, A., Favier, B., Cabanac, G., \& Labbé, C. (2021). The thin ret(raction) line: Biomedical journal responses to incorrect nontargeting nucleotide sequence reagents in human gene knockdown publications. Scientometrics, 126(4), 3513-3534. https://doi.org/10.1007/s11192-021-03871-9

Campos-Varela, I., \& Ruano-Raviña, A. (2019). Misconduct as the main cause for retraction. A descriptive study of retracted publications and their authors. Gaceta Sanitaria, 33(4), 356-360. https://doi.org/10.1016/j.gaceta.2018.01.009

Campos-Varela, I., Villaverde-Castañeda, R., \& Ruano-Raviña, A. (2020). Retraction of publications: A study of biomedical journals retracting publications based on impact factor and journal category. Gaceta Sanitaria, 34(5), 430-434.

https://doi.org/10.1016/j.gaceta.2019.05.008

Candal-Pedreira, C., Ruano-Raviña, A., Fernández, E., Ramos, J., Campos-Varela, I., \& Pérez-Ríos, M. (2020). Does retraction after misconduct have an impact on citations? A pre-post study. BMJ Global Health, 5(11), e003719. https://doi.org/10.1136/bmjgh$\underline{2020-003719}$

Carey, L. C., Seth, A., Woolley, M., \& Woolley, K. L. (2016). Avoiding high-risk authors: Should Corporate Integrity Agreements recommend searching the retracted publications database? Annual Meeting of the International Society for Medical Publication Professionals, Current Medical Research and Opinion, 32(sup1), S5-S6. https://doi.org/10.1185/03007995.2016.1152850

Casadevall, A., Steen, R. G., \& Fang, F. C. (2014). Sources of error in the retracted scientific literature. The FASEB Journal, 28(9), 3847-3855.

https://doi.org/10.1096/fj.14-256735

Cassão, B. D., Herbella, F. A. M., Schlottmann, F., \& Patti, M. G. (2018). Retracted articles in surgery journals. What are surgeons doing wrong? Surgery, 163(6), 1201-1206. https://doi.org/10.1016/j.surg.2018.01.015

Chambers, L. M., Michener, C. M., \& Falcone, T. (2019). Plagiarism and data falsification are the most common reasons for retracted publications in obstetrics and gynaecology. BJOG: An International Journal of Obstetrics and Gynaecology, 126(9), 1134-1140. https://doi.org/10.1111/1471-0528.15689 
Chauvin, A., de Villelongue, C., Pateron, D., \& Yordanov, Y. (2019). A systematic review of retracted publications in emergency medicine. European Journal of Emergency Medicine, 26(1), 19-23. https://doi.org/10.1097/MEJ.0000000000000491

Chen, C., Hu, Z., Milbank, J., \& Schultz, T. (2013). A visual analytic study of retracted articles in scientific literature. Journal of the American Society for Information Science and Technology, 64(2), 234-253. https://doi.org/10.1002/asi.22755

Chen, W., Xing, Q. R., Wang, H., \& Wang, T. (2018). Retracted publications in the biomedical literature with authors from mainland China. Scientometrics, 114(1), 217227. https://doi.org/10.1007/s11192-017-2565-X

Cheng, Y. Y., Parulian, N., Hsiao, T. K., Dinh, L., Sarol, J., \& Schneider, J. (2019). ReTracker: Actively and automatically matching retraction metadata in Zotero. Annual Meeting of the Association for Information Science and Technology, 56(1), 372-376. https://doi.org/10.1002/pra2.32

Claxton, L. D. (2005). Scientific authorship: Part 1. A window into scientific fraud? Mutation Research/Reviews in Mutation Research, 589(1), 17-30. https://doi.org/10.1016/i.mrrev.2004.07.003

Cokol, M., lossifov, I., Rodriguez-Esteban, R., \& Rzhetsky, A. (2007). How many scientific papers should be retracted? EMBO Reports, 8(5), 422-423.

https://doi.org/10.1038/sj.embor.7400970

Cokol, M., Ozbay, F., \& Rodriguez-Esteban, R. (2008). Retraction rates are on the rise. EMBO Reports, 9(1), 2. https://doi.org/10.1038/sj.embor.7401143

Cole, G. D., Nowbar, A. N., Mielewczik, M., Shun-Shin, M. J., \& Francis, D. P. (2015). Frequency of discrepancies in retracted clinical trial reports versus unretracted reports: Blinded case-control study. BMJ, 351, h4708. https://doi.org/10.1136/bmj.h4708 (Correction published September 25, 2015, BMJ, 351, p. h5134, https://doi.org/10.1136/bmj.h5134)

Copiello, S. (2019). On the skewness of journal self-citations and publisher self-citations: Cues for discussion from a case study. Learned Publishing, 32(3), 249-258. https://doi.org/10.1002/leap.1235

Copiello, S. (2020). Other than detecting impact in advance, alternative metrics could act as early warning signs of retractions: Tentative findings of a study into the papers retracted by PLoS ONE. Scientometrics, 125(3), 2449-2469. https://doi.org/10.1007/s11192-020-03698-w

Cor, K., \& Sood, G. (2018). Propagation of error: Approving citations to problematic research [preprint]. http://www.gsood.com/research/papers/error.pdf

Cortegian,i A., Catalisano, G., Ippolito, M., Giarratano, A., Absalom, A. R., \& Einav, S. (2021). Retracted papers on SARS-CoV-2 and COVID-19. British Journal of Anaesthesia, 126(4), e155-e156. https://doi.org/10.1016/j.bja.2021.01.008

Coudert, F. X. (2019). Correcting the scientific record: Retraction practices in chemistry and materials science. Chemistry of Materials, 31(10), 3593-3598. https://doi.org/10.1021/acs.chemmater.9b00897 (Correction published August 30, 2019, Chemistry of Materials, 31(18), p. 7800, https://doi.org/10.1021/acs.chemmater.9b03415) 
Cox, A., Craig, R., \& Tourish, D. (2018). Retraction statements and research malpractice in economics. Research Policy, 47(5), 924-935.

https://doi.org/10.1016/j.respol.2018.02.016

Craig, R., Cox, A., Tourish, D., \& Thorpe, A. (2020). Using retracted journal articles in psychology to understand research misconduct in the social sciences: What is to be done? Research Policy, 49(4), 103930. https://doi.org/10.1016/j.respol.2020.103930

Craig, R., Pelosi, A., \& Tourish, D. (2021). Research misconduct complaints and institutional logics: The case of Hans Eysenck and the British Psychological Society. Journal of Health Psychology, 26(2), 296-311.

https://doi.org/10.1177/1359105320963542

Dal-Ré, R. (2019). Analysis of retracted articles on medicines administered to humans. British Journal of Clinical Pharmacology, 85(9), 2179-2181. https://doi.org/10.1111/bcp.14021

Dal-Ré, R. (2020). Analysis of biomedical Spanish articles retracted between 1970 and 2018. Medicina Clinica, 154(4), 125-130. https://doi.org/10.1016/j.medcli.2019.04.018

Dal-Ré, R., \& Ayuso, C. (2019). Reasons for and time to retraction of genetics articles published between 1970 and 2018. Journal of Medical Genetics, 56, 734-740. https://doi.org/10.1136/jmedgenet-2019-106137 (Correction published June 1, 2020, Journal of Medical Genetics, 57, p. 435-436.)

Dal-Ré, R., \& Ayuso, C. (2021). For how long and with what relevance do genetics articles retracted due to research misconduct remain active in the scientific literature.

Accountability in Research, 28(5), 280-296.

https://doi.org/10.1080/08989621.2020.1835479

Dal-Ré, R., \& Morell, F. (2021). [Spanish] La pandemia de la COVID-19 modifica el sistema de publicación científica [The COVID-19 pandemic changes the scientific publication system]. Archivos de Bronconeumologia, 57(S1), 17-18.

https://doi.org/10.1016/j.arbres.2020.10.010

Damineni, R. S., Sardiwal, K. K., Waghle, S. R., \& Dakshyani, M. B. (2015). A comprehensive comparative analysis of articles retracted in 2012 and 2013 from the scholarly literature. Journal of International Society of Preventive and Community Dentistry, 5(1), 19-23. https://doi.org/10.4103/2231-0762.151968

Davis, P. M. (2012). The persistence of error: A study of retracted articles on the Internet and in personal libraries. Journal of the Medical Library Association, 100(3), 184-189. https://doi.org/10.3163/1536-5050.100.3.008 (Correction published October 2012, Journal of the Medical Library Association : JMLA, 100(4), p. 329, https://doi.org/10.3163/1536-5050.100.4.027)

de Almeida, R. M. V. R., Catelani, F., Fontes-Pereira, A. J., \& de Souza, G. N. (2016). Retractions in general and internal medicine in a high-profile scientific indexing database [Retratações em medicina geral e interna em um indexador científico de alta visibilidade]. Sao Paulo Medical Journal, 134(1), 74-78. https://doi.org/10.1590/1516$\underline{3180.2014 .00381601}$

de Vasconcelos, S. M. R., \& Roig, M. (2015). Prior publication and redundancy in contemporary science: Are authors and editors at the crossroads? Science and Engineering Ethics, 21(5), 1367-1378. https://doi.org/10.1007/s11948-014-9599-8 
(Erratum published November 18, 2014, Science and Engineering Ethics, 21, p. 1379, https://doi.org/10.1007/s11948-014-9610-4)

Decullier, E., Huot, L., \& Maisonneuve, H. (2014). What time-lag for a retraction search on PubMed? BMC Research Notes, 7(1), 395.https://doi.org/10.1186/1756-0500-7-395

Decullier, E., Huot, L., Samson, G., \& Maisonneuve, H. (2013). Visibility of retractions: A cross-sectional one-year study. BMC Research Notes, 6(1), 238.

https://doi.org/10.1186/1756-0500-6-238

Decullier, E., \& Maisonneuve, H. (2018). Correcting the literature: Improvement trends seen in contents of retraction notices. BMC Research Notes, 11(1), 490.

https://doi.org/10.1186/s13104-018-3576-2

Dinh, L., Sarol, J., Cheng, Y. Y., Hsiao, T. K., Parulian, N., \& Schneider, J. (2019). Systematic examination of pre- and post-retraction citations. Proceedings of the Association for Information Science and Technology, 56(1), 390-394. https://doi.org/10.1002/pra2.35

Dobránszki, J., \& Teixeira da Silva, J. A. (2019). Corrective factors for author- and journalbased metrics impacted by citations to accommodate for retractions. Scientometrics, 121(1), 387-398. https://doi.org/10.1007/s11192-019-03190-0

Dollfuß, H. (2015). [German] Analyse zurückgezogener Publikationen in der bibliografischen Datenbank Web of Science von 2004 bis 2014 [Analysis of retracted publications in the bibliographic database Web of Science between 2004 and 2014]. GMS Medizin — Bibliothek — Information, 15(1-2). https://doi.org/10.3205/mbi000336

Dougherty, M. V. (2018). Correcting the Scholarly Record for Research Integrity: In the Aftermath of Plagiarism. Springer International Publishing. https://doi.org/10.1007/9783-319-99435-2

Dougherty, M. V. (2020). Disguised Academic Plagiarism: A Typology and Case Studies for Researchers and Editors. Springer. https://doi.org/10.1007/978-3-030-46711-1

Drimer-Batca, D., laccarino, J. M., \& Fine, A. (2019). Status of retraction notices for biomedical publications associated with research misconduct. Research Ethics, 15(2), 1-5. https://doi.org/10.1177/1747016118820496

Drury, N. E., \& Karamanou, D. M. (2009). Citation of retracted articles: A call for vigilance. The Annals of Thoracic Surgery, 87(2), 670.

https://doi.org/10.1016/j.athoracsur.2008.07.108

Duggar, D. C., Christopher, K. A., Tucker, B. E., Jones, D. A., Watson, M. M., Puckett, M., \& Wood, B. (1995). Promoting an awareness of retractions: The Louisiana State University Medical Center in Shreveport experience. Medical Reference Services Quarterly, 14(1), 17-32. https://doi.org/10.1300/J115V14N01 03

Dutta Majumder, P., Raman, R., Krishnan, T., \& George, R. (2021). Analysis of retracted articles in the ophthalmic literature [Online First]. Eye, 1-5. https://doi.org/10.1038/s41433-021-01438-9

Elango, B., \& Kozak, M. (2020). Retractions among Indian scientific authors: Multiple offenders. Research Square. https://doi.org/10.21203/rs.3.rs-28552/v1 
Elango, B. (2021). Retracted articles in the biomedical literature from Indian authors. Scientometrics, 126, 3965-3981. https://doi.org/10.1007/s11192-021-03895-1

Elango, B., Kozak, M., \& Rajendran, P. (2019). Analysis of retractions in Indian science. Scientometrics, 119(2), 1081-1094. https://doi.org/10.1007/s11192-019-03079-y

Elia, N., Wager, E., \& Tramèr, M. R. (2014). Fate of articles that warranted retraction due to ethical concerns: A descriptive cross-sectional study. PLoS ONE, 9(1), e85846. https://doi.org/10.1371/journal.pone.0085846

Elisha, E., Guetzkow, J., Shir-Raz, Y., \& Ronel, N. (2021). Retraction of scientific papers: The case of vaccine research [Online First]. Critical Public Health. https://doi.org/10.1080/09581596.2021.1878109

El-Tahan, M. R. (2019). Can the similarity index predict the causes of retractions in highimpact anesthesia journals? A bibliometric analysis. Saudi Journal of Anaesthesia, 13(Suppl 1), S2-S8.https://doi.org/10.4103/sja.SJA 70918

Erfanmanesh, M., \& Teixeira da Silva, J. A. (2019). Is the soundness-only quality control policy of open access mega journals linked to a higher rate of published errors? Scientometrics, 120(2), 917-923. https://doi.org/10.1007/s11192-019-03153-5

Faggion, C. M. Jr., Ware, R. S., Bakas, N., \& Wasiak, J. (2018). An analysis of retractions of dental publications. Journal of Dentistry, 79, 19-23. https://doi.org/10.1016/j.jdent.2018.09.002

Fanelli, D. (2013). Why growing retractions are (mostly) a good sign. PLoS Medicine, 10(12), e1001563. https://doi.org/10.1371/journal.pmed.1001563

Fanelli, D., Costas, R., \& Larivière, V. (2015). Misconduct policies, academic culture and career stage, not gender or pressures to publish, affect scientific integrity. PLoS ONE, 10(6), e0127556. https://doi.org/10.1371/journal.pone.0127556

Fanelli, D., loannidis, J. P. A., \& Goodman, S. (2018). Improving the integrity of published science: An expanded taxonomy of retractions and corrections. European Journal of Clinical Investigation, 48, e12898. https://doi.org/10.1111/eci.12898

Fanelli, D., \& Moher, D. (2019). What difference do retractions make? An estimate of the epistemic impact of retractions on recent meta-analyses. biorXiv. https://doi.org/10.1101/734137

Fang, F. C., \& Casadevall, A. (2011). Retracted science and the retraction index. Infection and Immunity, 79(10), 3855-3859. https://doi.org/10.1128/IAI.05661-11

Fang, F. C., Steen, R. G., \& Casadevall, A. (2012). Misconduct accounts for the majority of retracted scientific publications. Proceedings of the National Academy of Sciences, 109(42), 17028-17033. https://doi.org/10.1073/pnas.1212247109 (Erratum published January 15, 2013, Proceedings of the National Academy of Sciences, 110(3), p. 1137, https://doi.org/10.1073/pnas.1220649110)

Feng, L., Yuan, J., \& Yang, L. (2020). An observation framework for retracted publications in multiple dimensions. Scientometrics, 125(2), 1445-1457.

https://doi.org/10.1007/s11192-020-03702-3

Fiore, M., Alfieri, A., Pace, M. C., Simeon, V., Chiodini, P., Leone, S., Wirz, S., Cuomo, A., Stoia, V., \& Cascella, M. (2021). A scoping review of retracted publications in 
anesthesiology. Saudi Journal of Anaesthesia, 15(2), 179-188.

https://doi.org/10.4103/sja.sja 111020

Foo, J. Y. A. (2013). Tainted publications and their authors' publication trend profiles: A study on Singapore from the year 2004 to 2010. In Ethics Research Compendium (pp. 109-117). Nova Science Publishers.

Foo, J. Y. A. (2011). A retrospective analysis of the trend of retracted publications in the field of biomedical and life sciences. Science and Engineering Ethics, 17(3), 459-468. https://doi.org/10.1007/s11948-010-9212-8

Foo, J. Y. A., \& Tan, X. J. A. (2014). Analysis and implications of retraction period and coauthorship of fraudulent publications. Accountability in Research, 21(3), 198-210. https://doi.org/10.1080/08989621.2013.848799

Foo, J. Y. A., \& Wilson, S. J. (2012). An analysis on the research ethics cases Managed by the Committee on Publication Ethics (COPE) Between 1997 and 2010. Science and Engineering Ethics, 18(4), 621-631. https://doi.org/10.1007/s11948-011-9273-3

Friedman, P. J. (1990). Correcting the literature following fraudulent publication. JAMA, 263(10), 1416-1419. https://doi.org/10.1001/jama.1990.03440100136019

Fu, Y., \& Schneider, J. (2020). Towards knowledge maintenance in scientific digital libraries with the keystone framework. ACM/IEEE Joint Conference on Digital Libraries, 217226. https://doi.org/10.1145/3383583.3398514

Fulton, A. S., Coates, A. M., Williams, M. T., Howe, P. R. C., \& Hill, A. M. (2015). Persistent citation of the only published randomised controlled trial of Omega-3 supplementation in chronic obstructive pulmonary disease six years after its retraction. Publications, 3(1), 17-26. https://doi.org/10.3390/publications3010017 (Correction published July 27, 2018, Publications, 6(3), p. 35, https://doi.org/10.3390/publications6030035)

Furman, J. L., Jensen, K., \& Murray, F. (2012). Governing knowledge in the scientific community: Exploring the role of retractions in biomedicine. Research Policy, 41(2), 276-290. https://doi.org/10.1016/j.respol.2011.11.001

Gabehart, M. E. (2005). An Analysis of Citations to Retracted Articles in the Scientific Literature [Master's thesis, University of North Carolina at Chapel Hill]. https://ils.unc.edu/MSpapers/3050.pdf

Galbraith, K. L. (2017). Life after research misconduct. Journal of Empirical Research on Human Research Ethics, 12(1), 26-32. https://doi.org/10.1177/1556264616682568

Gammon, E. (2009). A Case Study Approach to Estimating the Burden of Research Misconduct in Publicly Funded Medical Research [Doctoral dissertation, University of Texas]. https://digitalcommons.library.tmc.edu/dissertations/AAl3350093/

Gammon, E., \& Franzini, L. (2013). Research misconduct oversight: Defining case costs. Journal of Health Care Finance, 40(2), 75-99.

Gamstaetter T. (2020). Retractions of papers by German biomedical authors [preregistration]. Open Science Framework. https://doi.org/10.17605/OSF.IO/BUA5W

Garfield, E., \& Welljams-Dorof, A. (1990). The impact of fraudulent research on the scientific literature: The Stephen E. Breuning case. JAMA, 263(10), 1424-1426. https://doi.org/10.1001/jama.1990.03440100144021 
Gasparyan, A. Y., Ayvazyan, L., Akazhanov, N. A., \& Kitas, G. D. (2014). Self-correction in biomedical publications and the scientific impact. Croatian Medical Journal, 55(1), 6172. https://doi.org/10.3325/cmj.2014.55.61

Gaston, T. E., Ounsworth, F., Senders, T., Ritchie, S., \& Jones, E. (2020). Factors affecting journal submission numbers: Impact factor and peer review reputation. Learned Publishing, 33(2), 154-162. https://doi.org/10.1002/leap.1285

Gayle, A., \& Shimaoka, M. (2017). Public response to scientific misconduct: Assessing changes in public sentiment toward the Stimulus-Triggered Acquisition of Pluripotency (STAP) cell Case via Twitter. JMIR Public Health and Surveillance, 3(2), e21. https://doi.org/10.2196/publichealth.5980

Genot, E., \& Olsson, E. J. (2021). The dissemination of fake science: On the ranking of retracted articles in Google [forthcoming]. In Bernecker S, Flowerree A K, \& Grundmann T (Eds.), The Epistemology of Fake News. Oxford University Press. https://portal.research.lu.se/ws/files/94696954/Dissemination of Scientific Fake New s.pdf

Glasnovic, A., Krajna, T., \& Petrak, J. (2019). Retracted articles by Croatian authors: A case study. European Science Editing, 45(4), 85-88. https://doi.org/10.20316/ESE.2019.45.19009

Gray, R., Al-Ghareeb, A., \& McKenna, L. (2019). Why articles continue to be cited after they have been retracted: An audit of retraction notices. International Journal of Nursing Studies, 90, 11-12. https://doi.org/10.1016/j.jjnurstu.2018.10.003

Gray, R., Al-Ghareeb, A., Davis, J., McKenna, L., \& Hillel, S. (2018). Inclusion of nursing trials in systematic reviews after they have been retracted: Does it happen and what should we do? International Journal of Nursing Studies, 79, 154. https://doi.org/10.1016/j.ijnurstu.2017.12.006

Greitemeyer, T. (2014). Article retracted, but the message lives on. Psychonomic Bulletin \& Review, 21(2), 557-561. https://doi.org/10.3758/s13423-013-0500-6

Greitemeyer, T., \& Sagioglou, C. (2015). Does exonerating an accused researcher restore the researcher's credibility? PLoS ONE, 10(5), e0126316. https://doi.org/10.1371/journal.pone.0126316

Grey, A., Avenell, A., Gamble, G., \& Bolland, M. (2020). Assessing and raising concerns about duplicate publication, authorship transgressions and data errors in a body of preclinical research. Science and Engineering Ethics, 26, 2069-2096. https://doi.org/10.1007/s11948-019-00152-w

Grey, A., Avenell, A., \& Bolland, M. (2021). Timeliness and content of retraction notices for publications by a single research group [Online First]. Accountability in Research. https://doi.org/10.1080/08989621.2021.1920409

Grey, A., Bolland, M., Gamble, G., \& Avenell, A. (2019). Quality of reports of investigations of research integrity by academic institutions. Research Integrity and Peer Review, 4(1), 3. https://doi.org/10.1186/s41073-019-0062-x

Grieneisen, M. L., \& Zhang, M. (2012). A comprehensive survey of retracted articles from the scholarly literature. PLoS ONE, 7(10), e44118.

https://doi.org/10.1371/journal.pone.0044118 
Gupta, L., Gasparyan, A. Y., Misra, D. P., Agarwal, V., Zimba, O., \& Yessirkepov, M. (2020). Information and misinformation on COVID-19: A cross-sectional survey study. Journal of Korean Medical Science, 35(27), e256. https://doi.org/10.3346/JKMS.2020.35.E256

Gutiérrez, S. A., Barbosa, H. J., Cuero, M. S., Duarte, E. J., Gaitán, F. E., Lozano, J. L., Meneses, A., Olaya, J., Pacheco, G. F., Rodríguez, C. C., Clavijo, J. A., \& Vallejo, G. A. (2016). [Spanish] La retractación y la corrección de la literatura científica para conservar la integridad y la confianza en la ciencia: Un análisis de retractaciones de publicaciones biomédicas de libre acceso en PubMed, 1959-2015 [Retraction and correction of scientific literature for conserving the integrity of and confidence in science: An analysis of retractions in open access biomedical publications in PubMed, 1959-2015]. Revista de La Academia Colombiana de Ciencias Exactas, Físicas y Naturales, 40(157), 568-579. https://doi.org/10.18257/raccefyn.399

Hagberg, J. M. (2020). The unfortunately long life of some retracted biomedical research publications. Journal of Applied Physiology, 128(5), 1381-1391. https://doi.org/10.1152/japplphysiol.00003.2020

Halevi, G. (2020). Why articles in arts and humanities are being retracted? Publishing Research Quarterly, 36, 55-62. https://doi.org/10.1007/s12109-019-09699-9

Hamilton, D. G. (2019). Continued citation of retracted radiation oncology literature-do we have a problem? International Journal of Radiation Oncology, Biology, Physics, 103(5), 1036-1042. https://doi.org/10.1016/j.jirobp.2018.11.014

Han, J. \& Li, Z. (2018). How metrics-based academic evaluation could systematically induce academic misconduct: A case study. East Asian Science, Technology and Society: An International Journal, 12(2), 165-179. https://doi.org/10.1215/18752160$\underline{4275144}$

Haunschild, R., \& Bornmann, L. (2021). Can tweets be used to detect problems early with scientific papers? A case study of three retracted COVID-19/SARS-CoV-2 papers. Scientometrics, 126, 5181-5199. https://doi.org/10.1007/s11192-021-03962-7

He, T. (2013). Retraction of global scientific publications from 2001 to 2010 . Scientometrics, 96(2), 555-561. https://doi.org/10.1007/s11192-012-0906-3

Heibi, I., \& Peroni, S. (2021a). A protocol to gather, characterize and analyze incoming citations of retracted articles. arXiv. http://arxiv.org/abs/2106.01781

Heibi, I., \& Peroni, S. (2021b). A qualitative and quantitative citation analysis toward retracted articles: A case of study. arXiv. http://arxiv.org/abs/2012.11475

Heibi, I., \& Peroni, S. (2020). Methodology data of "A qualitative and quantitative citation analysis toward retracted articles: A case of study" (version 2). Zenodo.

https://doi.org/10.5281/zenodo.4323221

Hein, J., Zobrist, R., Konrad, C., \& Schuepfer, G. (2012). Scientific fraud in 20 falsified anesthesia papers: Detection using financial auditing methods. Der Anaesthesist, 61(6), 543-549. https://doi.org/10.1007/s00101-012-2029-x

Hernon, P., \& Altman, E. (1995). Misconduct in academic research: Its implications for the service quality provided by university libraries. The Journal of Academic Librarianship, 21(1), 27-37. https://doi.org/10.1016/0099-1333(95)90151-5 
Herrera-Peco, I., Santillán-García, A., Morán, J. M., Goodman-Casanova, J. M., \& CuestaLozano, D. (2020). The evidence-based practice silent enemy: Retracted articles and their use in systematic reviews. Healthcare, 8(4), 465.

https://doi.org/10.3390/healthcare8040465

Hesselmann, F., Graf, V., Schmidt, M., \& Reinhart, M. (2017). The visibility of scientific misconduct: A review of the literature on retracted journal articles. Current Sociology, 65(6), 814-845. https://doi.org/10.1177/0011392116663807

Hesselmann, F., \& Reinhart, M. (2020). Cycles of invisibility: The limits of transparency in dealing with scientific misconduct. Social Studies of Science, 51(3), 414-438. https://doi.org/10.1177/0306312720975201

Hesselmann, F. (2019). Punishing crimes of the mind: Sanctions for scientific misconduct as a case for the cultural theory of punishment. Theoretical Criminology, 23(4), 527544. https://doi.org/10.1177/1362480618756365

Hesselmann, F., \& Reinhart, M. (2019). Science means never having to say you're sorry? Apologies for scientific misconduct. Science Communication, 41(5), 552-579. https://doi.org/10.1177/1075547019860848

Hilgard, J., \& Jamieson, K. H. (2017). Science as "broken" versus science as "selfcorrecting": How retractions and peer-review problems are exploited to attack science. In The Oxford Handbook of the Science of Science Communication (pp. 85-92). https://doi.org/10.1093/oxfordhb/9780190497620.013.9

Horbach, S. P. J. M., \& Halffman, W. (2019). The ability of different peer review procedures to flag problematic publications. Scientometrics, 118(1), 339-373. https://doi.org/10.1007/s11192-018-2969-2

Horton, J., Krishna Kumar, D., \& Wood, A. (2020). Detecting academic fraud using Benford law: The case of Professor James Hunton. Research Policy, 49(8), 104084. https://doi.org/10.1016/j.respol.2020.104084

Hosseini, M., Hilhorst, M., de Beaufort, I., \& Fanelli, D. (2018). Doing the right thing: A qualitative investigation of retractions due to unintentional error. Science and Engineering Ethics, 24(1), 189-206. https://doi.org/10.1007/s11948-017-9894-2

Hu, G., \& Xu, S. B. (2020). Agency and responsibility: A linguistic analysis of culpable acts in retraction notices. Lingua, 247, 102954. https://doi.org/10.1016/j.lingua.2020.102954

Hu, G. (2017). Authorship of retraction notices: If names are not rectified, then language will not be in accord with truth. Publications, 5(2), 10.

https://doi.org/10.3390/publications5020010

Hubbard, D. E. (2010). Corrections in the chemical literature: Their number and nature. Science \& Technology Libraries, 29(1-2), 130-143. https://doi.org/10.1080/01942620903579427

Hughes, C. (1998). Academic medical libraries' policies and procedures for notifying library users of retracted scientific publications. Medical Reference Services Quarterly, 17(2), 37-42. https://doi.org/10.1300/J115v17n02 04 
Huh, S., Kim, S. Y., \& Cho, H. M. (2016). Characteristics of retractions from Korean medical journals in the KoreaMed database: A bibliometric analysis. PLoS ONE, 11(10), e0163588. https://doi.org/10.1371/journal.pone.0163588

Hussinger, K., \& Pellens, M. (2019a). Guilt by association: How scientific misconduct harms prior collaborators. Research Policy, 48(2), 516-530.

https://doi.org/10.1016/j.respol.2018.01.012

Hussinger, K., \& Pellens, M. (2019b). Scientific misconduct and accountability in teams. PLoS ONE, 14(5), e0215962. https://doi.org/10.1371/journal.pone.0215962

Hwang, K., \& Wu, X. (2018). Retracted or withdrawn publications in journals relating to plastic surgery. The Journal of Craniofacial Surgery, 29(5), 1114-1116. https://doi.org/10.1097/SCS.0000000000004326

Inoue, Y., \& Muto, K. (2016). Noncompliance with human subjects' protection requirements as a reason for retracting papers: Survey of retraction notices on medical papers published from 1981 to 2011. Accountability in Research, 23(2), 123-135. https://doi.org/10.1080/08989621.2015.1069713

Jan, R., Bano, S., Ikhlaq ur Rehman, S., \& Mehraj, M. (2018). Context analysis of top seven retracted articles: Should Retraction Watch revisit the list? Library Philosophy and Practice, 2016. https://digitalcommons.unl.edu/libphilprac/2016/

Jan, R., \& Zainab, T. (2018). The impact story of retracted articles Altmetric it! IEEE International Symposium on Emerging Trends and Technologies in Libraries and Information Services, 402-406. https://doi.org/10.1109/ETTLIS.2018.8485245

Jones, D. A., Watson, M. M., Comegys, M., Burnett, A., \& Tucker, B. E. (2003). Errata and retractions in electronic journals. Journal of Hospital Librarianship, 3(2), 19-27. https://doi.org/10.1300/J186v03n02 02

Kamali, N., Abadi, A. T. B., \& Rahimi, F. (2020). Plagiarism, fake peer-review, and duplication: Predominant reasons underlying retractions of Iran-affiliated scientific papers. Science and Engineering Ethics, 26(6), 3455-3463. https://doi.org/10.1007/s11948-020-00274-6

Kamali, N., Rahimi, F., \& Abadi, A. T. B. (2020). Learning from retracted papers authored by the highly cited Iran-affiliated researchers: Revisiting research policies and a key message to Clarivate Analytics. Research Square. https://doi.org/10.21203/rs.3.rs$113269 / \mathrm{v} 1$

Kang, H. D., \& Lee, J. J. (2016). Scientific celebrity, competition, and knowledge creation: The case of stem cell research in South Korea. Journal of Engineering and Technology Management - JET-M, 39, 26-44. https://doi.org/10.1016/j.jengtecman.2016.01.001

Karabag, S. F., \& Berggren, C. (2016). Misconduct, marginality and editorial practices in management, business and economics journals. PLoS ONE, 11(7), e0159492. https://doi.org/10.1371/journal.pone.0159492

Karabag, S. F., \& Berggren, C. (2012). Retraction, dishonesty and plagiarism: Analysis of a crucial issue for academic publishing, and the inadequate responses from leading journals in economics and management disciplines. Journal of Applied Economics and Business Research, 2(3), 172-183. 
Kardeş, S., Levack, W., Özkuk, K., Atmaca Aydın, E., \& Seringeç Karabulut, S. (2020). Retractions in rehabilitation and sport sciences journals: A systematic review. Archives of Physical Medicine and Rehabilitation, 101(11), 1980-1990.

https://doi.org/10.1016/j.apmr.2020.03.010

Khamis, A. M., Hakoum, M. B., Bou-Karroum, L., Habib, J. R., Ali, A., Guyatt, G., El-Jardali, F., \& Akl, E. A. (2017). Requirements of health policy and services journals for authors to disclose financial and non-financial conflicts of interest: A cross-sectional study. Health Research Policy and Systems, 15(1), 80. https://doi.org/10.1186/s12961-017$\underline{0244-2}$

Kim, S. Y., Yi, H. J., Cho, H. M., \& Huh, S. (2019). How many retracted articles indexed in KoreaMed were cited 1 year after retraction notification. Science Editing, 6(2), 122127. https://doi.org/10.6087/kcse.172

King, E.G., Oransky, I., Sachs, T. E., Farber, A., Flynn, D. B., Abritis, A. J., Kalish, J. A., \& Siracuse, J. J. (2018). Analysis of retracted articles in the surgical literature. American Journal of Surgery, 216(5), 851-855. https://doi.org/10.1016/j.amjsurg.2017.11.033

Kochan, C. A., \& Budd, J. M. (1992). The persistence of fraud in the literature: The Darsee case. Journal of the American Society for Information Science, 43(7), 488-493. https://doi.org/10.1002/(SICI)1097-4571(199208)43:7<488::AID-ASI3>3.0.CO;2-7

Kodvanj, I., Homolak, J., Virag, D., \& Trkulja, V. (2020). Publishing of COVID-19 preprints in peer-reviewed journals, preprinting trends, public discussion and quality issues. bioRxiv. https://doi.org/10.1101/2020.11.23.394577

Korpela, K. M. (2010). How long does it take for the scientific literature to purge itself of fraudulent material?: The Breuning case revisited. Current Medical Research and Opinion, 26(4), 843-847. https://doi.org/10.1185/03007991003603804

Kuroki, T., \& Ukawa, A. (2018). Repeating probability of authors with retracted scientific publications. Accountability in Research, 25(4), 212-219.

https://doi.org/10.1080/08989621.2018.1449651

Labbe, C., Grima, N., Gautier, T., Favier, B., \& Byrne, J. A. (2019). Semi-automated factchecking of nucleotide sequence reagents in biomedical research publications: The Seek \& Blastn tool. PLoS ONE, 14(3), e0213266.

https://doi.org/10.1371/journal.pone.0213266

LaCroix, T., Geil, A., \& O'Connor, C. (2021). The dynamics of retraction in epistemic networks. Philosophy of Science, 88(3). https://doi.org/10.1086/712817

Lee, K. H., Kim, J. S., Hong, S. H., Seong, D., Choi, Y. R., Ahn, Y. T., Kim, K. S., Kim, S. E., Lee, S., Sim, W., Kim, D., Jun, B., Yang, J. W., Yon, D. K., Lee, S. W., Kim, M.S., Dragioti, E., Li, H., Jacob, L., ... Smith, L. (2020). Risk factors of COVID-19 mortality: A systematic review of current literature and lessons from recent retracted articles. European Review for Medical and Pharmacological Sciences, 24(24), 13089-13097. https://doi.org/10.26355/eurrev 20201224216

Lelgemann, M., \& Sauerland, S. (2010). Fraudulent studies, unpublished data and their effect on the development of guidelines and evidence-based recommendations Gefälschte Studien und nicht publizierte Daten: Auswirkung auf die Erarbeitung von Leitlinien und evidenzbasierten Empfehlungen. Zeitschrift Fur Evidenz, Fortbildung 
Und Qualitat Im Gesundheitswesen, 104(4), 284-291.

https://doi.org/10.1016/j.zefq.2010.03.035

Lesk, M., Mattern, J. B., \& Moulaison, S. H. (2019). Are papers with open data more credible? An analysis of open data availability in retracted PLoS articles. International Conference on Information, LNCS 11420, 154-161. https://doi.org/10.1007/978-3-03015742-5 14

Li, G., Kamel, M., Jin, Y., Xu, M. K., Mbuagbaw, L., Samaan, Z., Levine, M. A., \& Thabane, L. (2018). Exploring the characteristics, global distribution and reasons for retraction of published articles involving human research participants: A literature survey. Journal of Multidisciplinary Healthcare, 11, 39-47. https://doi.org/10.2147/JMDH.S151745

Lievore, C., Rubbo, P., dos Santos, C. B., Picinin, C. T., \& Pilatti, L. A. (2021). Research ethics: A profile of retractions from world class universities [Online First].

Scientometrics. https://doi.org/10.1007/s11192-021-03987-y

Lin, W. Y. C. (2020). Self-plagiarism in academic journal articles: From the perspectives of international editors-in-chief in editorial and COPE case. Scientometrics, 123(1), 299319. https://doi.org/10.1007/s11192-020-03373-0

Liu, W., \& Lei, L. (2021). Retractions in the Middle East from 1999 to 2018: A bibliometric analysis. Scientometrics, 126, 4687-4700. https://doi.org/10.1007/s11192-021-03919$\underline{\mathrm{w}}$

Liu, X., \& Chen, X. (2021). Authors' noninstitutional emails and their correlation with retraction. Journal of the Association for Information Science and Technology, 72(4), 473-477. https://doi.org/10.1002/asi.24419

Lock, S. (1988). Misconduct in medical research: Does it exist in Britain? British Medical Journal, 297(6662), 1531-1535. https://doi.org/10.1136/bmj.297.6662.1531

Lu, S. F., Jin. G. Z., Uzzi, B., \& Jones, B. (2013). The retraction penalty: Evidence from the Web of Science. Scientific Reports, 3, 3146. https://doi.org/10.1038/srep03146

Luwel, M., van Eck, N. J., \& van Leeuwen, T. N. (2018). The Schön case: Analyzing in-text citations to papers before and after retraction. International Conference on Science and Technology Indicators, 23, 1025-1030. https://doi.org/10.31235/osf.io/c6mvs

Madhugiri, V. S., Nagella, A. B., \& Uppar, A. M. (2021). An analysis of retractions in neurosurgery and allied clinical and basic science specialties. Acta Neurochirurgica, 163(1), 19-30. https://doi.org/10.1007/s00701-020-04615-z

Madhugiri, V. S., Venkatesan, S., Dutt, A., \& Nagella, A. B. (2021). An estimation of the retraction gap across neurosurgery - a crevice or a chasm? [Online First]. World Neurosurgery.https://doi.org/10.1016/j.wneu.2021.05.067

Madlock-Brown, C. R., \& Eichmann, D. (2015). The (lack of) impact of retraction on citation networks. Science and Engineering Ethics, 21, 127-137.

https://doi.org/10.1007/s11948-014-9532-1

Malkov, D. (2020). Policy contamination network of the 1996 retracted article by Arnold et al. on estrogenic activity of environmental chemicals. Figshare.

https://doi.org/10.6084/m9.figshare.12318746.v1 
Malički, M., Utrobičić, A., \& Marušić, A.. (2019). Correcting duplicate publications: Follow up study of MEDLINE tagged duplications. Biochemia Medica, 29(1), 010201. https://doi.org/10.11613/BM.2019.010201

Mansourzadeh, M. J., Ghazimirsaeid, J., Motamedi, N., Najafi, A., Abdullahi Abubakar, A., \& Dehdarirad, H. (2021). A survey of Iranian retracted publications indexed in PubMed. Iranian Journal of Public Health, 50(1), 188-194. https://doi.org/10.18502/ijph.v50i1.5086

Marasović, T., Utrobiçić, A., \& Marusiić, A. (2018). Transparency of retracting and replacing articles. Lancet, 391(10127), 1244-1245. https://doi.org/10.1016/S0140$\underline{6736(18) 30487-2}$

Marco-Cuenca, G., Salvador-Oliván, J. A., \& Arquero-Avilés, R. (2019). [Spanish] Ética en la publicación científica biomédica. Revisión de las publicaciones retractadas en España [Ethics in biomedical scientific publication. A review of the publications retracted in Spain]. Profesional de la Información, 28(2), e280222. https://doi.org/10.3145/epi.2019.mar.22

Marco-Cuenca, G., Salvador-Oliván, J. A., \& Arquero-Avilés, R. (2021). Fraud in scientific publications in the European Union. An analysis through their retractions. Scientometrics, 126, 5143-5164. https://doi.org/10.1007/s11192-021-03977-0

Marco-Cuenca, G., Salvador-Oliván, J. A., Arquero-Avilés, R., Faggiolani, C., \& Siso-Calvo, B. (2021). [Spanish] Scientific publications of affiliated authors in Italy retracted due to fraud. Review and analysis. JLIS.It, 12(2), 23-38. https://doi.org/10.4403/jlis.it-12711

Marcus, A. (2018). A scientist's fraudulent studies put patients at risk. Science, 362(6413), 394. https://doi.org/10.1126/science.362.6413.394-a

Markowitz, D. M., \& Hancock, J. T. (2014). Linguistic traces of a scientific fraud: The case of Diederik Stapel. PLoS ONE, 9(8), e105937. https://doi.org/10.1371/journal.pone.0105937

Markowitz, D. M., \& Hancock, J. T. (2016). Linguistic obfuscation in fraudulent science. Journal of Language and Social Psychology, 35(4), 435-445.

https://doi.org/10.1177/0261927X15614605

Marret, E., Elia, N., Dahl, J. B., McQuay, H. J., Møiniche, S., Moore, R. A., Straube, S., \& Tramèr, M. R. (2009). Susceptibility to fraud in systematic reviews: Lessons from the Reuben case. Anesthesiology, 111(6), 1279-1289.

https://doi.org/10.1097/ALN.0b013e3181c14c3d

Martin-Arribas, M. C., Martinez-Hervas, I., Rodriguez-Lozano, I., \& Arias-Diaz, J. (2014). [Spanish] Percepción del fraude científico en las revistas biomédicas españolas [Perception of scientific fraud in the Spanish biomedical journals]. Medicina Clinica, 143(12), 554-559. https://doi.org/10.1016/j.medcli.2014.03.036

Matas, J., Buljan, I., \& Marušić, A. (2020). Correcting the pandemic: Analysis of corrections to journal articles on COVID-19 and Ebola. Journal of Global Health, 10(2), 1-3. https://doi.org/10.7189/jogh.10.020105

McHugh, U. M., \& Yentis, S. M. (2019). An analysis of retractions of papers authored by Scott Reuben, Joachim Boldt and Yoshitaka Fujii. Anaesthesia, 74(1), 17-21. 
https://doi.org/10.1111/anae.14414 (Correction published December 4, 2018, Anaesthesia, 74(1), p. 113, https://doi.org/10.1111/anae.14515)

Meckfessel, M., \& Moehrle, S. (2017). Self-regulation of the academic accounting literature: The case of James Hunton. Research in Accounting Regulation, 29(1), 10-18. https://doi.org/10.1016/j.racreg.2017.04.002

Mena, J. D., Ndoye, M., Cohen, A. J., Kamal, P., \& Breyer, B. N. (2019). The landscape of urological retractions: The prevalence of reported research misconduct. BJU International, 124(1), 174-179. https://doi.org/10.1111/bju.14706

Meneses-Fernández, M. D., \& Santana-Hernández, J. D. (2020). [Spanish] Construcción periodística de la mala praxis científica: Análisis en diarios digitales [Journalistic construction of scientific malpractice: Analysis in digital news media]. Prisma Social, 31, 40-63. https://revistaprismasocial.es/article/view/3853

Meuschke, N., Stange, V., Schubotz, M., \& Gipp, B. (2018). HyPlag: A hybrid approach to academic plagiarism detection. ACM SIGIR Conference on Research \& Development in Information Retrieval, 1321-1324. https://doi.org/10.1145/3209978.3210177

Mine, S. (2019). Toward responsible scholarly communication and innovation: A survey of the prevalence of retracted articles on scholarly communication platforms. Annual Meeting of the Association for Information Science \& Technology, 56(1), 738-739. https://doi.org/10.1002/pra2.155

Misra, D. P., \& Agarwal, V. (2018). Systematic reviews: Challenges for their justification, related comprehensive searches, and implications. Journal of Korean Medical Science, 33(12), e92. https://doi.org/10.3346/jkms.2018.33.e92

Misra, D. P., Ravindran, V., \& Agarwal, V. (2018). Integrity of authorship and peer review practices: Challenges and opportunities for improvement. Journal of Korean Medical Science, 33(46), e287. https://doi.org/10.3346/jkms.2018.33.e287

Misra, D. P., \& Agarwal, V. (2020). Integrity of clinical research conduct, reporting, publishing, and post-publication promotion in rheumatology. Clinical Rheumatology, 39, 1049-1060. https://doi.org/10.1007/s10067-020-04965-0

Mistry, V., Grey, A., \& Bolland, M. J. (2019). Publication rates after the first retraction for biomedical researchers with multiple retracted publications. Accountability in Research, 26(5), 277-287. https://doi.org/10.1080/08989621.2019.1612244

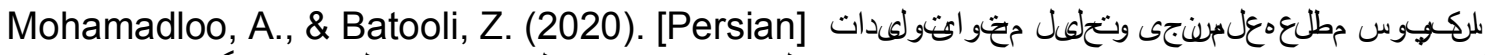
[A scientometric and content analysis of research output on "Retracted Papers" indexed in Scopus]. KAUMS Journal (FEYZ), 24(4), 446-461. http://feyz.kaums.ac.ir/article-1-4121-en.html

Molckovsky, A., Vickers, M. M., \& Tang, P. A. (2011). Characterization of published errors in high-impact oncology journals. Current Oncology, 18(1), 26-32. https://doi.org/10.3747/co.v18i1.707

Mongeon, P., \& Larivière, V. (2016). Costly collaborations: The impact of scientific fraud on co-authors' careers. Journal of the Association for Information Science and Technology, 67(3), 535-542. https://doi.org/10.1002/asi.23421 
Mongeon, P. (2014). [French] Les Rétractations et Leurs Conséquences sur la Carrière des Coauteurs: Analyse Bibliométrique des Fraudes et des Erreurs dans le Domaine Biomédical [Retractions and Their Consequences on the Careers of Co-authors: Bibliometric Analysis of Frauds and Errors in the Biomedical Field] [Master's thesis, Université de Montréal]. https://papyrus.bib.umontreal.ca/xmlui/handle/1866/10382

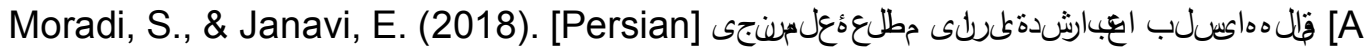
scientometrics study of Iranian retracted papers]. Iranian Journal of Information Processing and Management, 33(4), 1823-1844. http://jipm.irandoc.ac.ir/article-13557-en.html

Moradi, S. (2017). The altmetrics of retracted articles in biochemistry \& molecular biology. IFLA World Library and Information Congress. IFLA World Library and Information Congress. http://library.ifla.org/1607/

Morán, J. M., Santillán-García, A., \& Herrera-Peco, I. (2020). [Spanish] SCRUTATIOm: Cómo detectar literatura retractada incluida en revisiones sistemáticas y metaanálisis usando SCOPUSC y ZOTEROC [SCRUTATIOm: How to detect retracted literature included in systematics reviews and metaanalysis using SCOPUSC and ZOTEROC] [Online First]. Gaceta Sanitaria. https://doi.org/10.1016/j.gaceta.2020.06.012

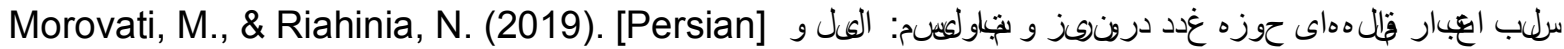
lo 1 [Retractions in endocrinology and metabolism journals: Causes and characteristics]. Journal of Health Administration, 22(4), 50-61. http://jha.iums.ac.ir/article-1-3111-en.html

Mott, A., Fairhurst, C., \& Torgerson, D. (2019). Assessing the impact of retraction on the citation of randomized controlled trial reports: An interrupted time-series analysis. Journal of Health Services Research \& Policy, 24(1), 44-51. https://doi.org/10.1177/1355819618797965

Moylan, E. C., \& Kowalczuk, M. K. (2016). Why articles are retracted: A retrospective crosssectional study of retraction notices at BioMed Central. BMJ Open, 6, e012047. https://doi.org/10.1136/bmjopen-2016-012047

Nagella, A. B., \& Madhugiri, V. S. (2020). Journal retraction rates and citation metrics: An ouroboric association? Cureus, 12(11), e11542.https://doi.org/10.7759/cureus.11542

Nair, S., Yean, C., Yoo, J., Leff, J., Delphin, E., \& Adams, D. C. (2020). Reasons for article retraction in anesthesiology: A comprehensive analysis [Raisons justifiant la rétractation d'un article en anesthésiologie: Une analyse exhaustive]. Canadian Journal of Anesthesia/Journal Canadien d'Anesthésie, 67, 57-63. https://doi.org/10.1007/s12630-019-01508-3

Nath, S. B., Marcus, S. C., \& Druss, B. G. (2006). Retractions in the research literature: Misconduct or mistakes? The Medical Journal of Australia, 185(3), 152-154. https://doi.org/10.5694/j.1326-5377.2006.tb00504.x

Neale, A. V., Dailey, R. K., \& Abrams, J. (2010). Analysis of citations to biomedical articles affected by scientific misconduct. Science and Engineering Ethics, 16(2), 251-261. https://doi.org/10.1007/s11948-009-9151-4

Neale, A. V., Northrup, J., Dailey, R. K., Marks, E., \& Abrams, J. (2007). Correction and use of biomedical literature affected by scientific misconduct. Science and Engineering Ethics, 13(1), 5-24. https://doi.org/10.1007/s11948-006-0003-1 
Nicholson, J. M., Uppala, A., Sieber, M., Grabitz, P., Mordaunt, M., \& Rife, S. C. (2021). Measuring the quality of scientific references in Wikipedia: An analysis of more than $115 \mathrm{M}$ citations to over 800000 scientific articles. FEBS Journal, 288(14), 4242-4248. https://doi.org/10.1111/febs.15608 (Correction published November 29, 2020, FEBS Journal)

Nogueira, T. E., Goncalves, A. S., Leles, C. R., Batista, A. C., \& Costa, L. R. (2017). A survey of retracted articles in dentistry. BMC Research Notes, 10(1), 253. https://doi.org/10.1186/s13104-017-2576-y

Okada, E. (2018). Knowledge corruption and governance in academic knowledge-intensive organizations: The case of molecular mutations research. Journal of Public Affairs, 18, e1698. https://doi.org/10.1002/pa.1698

Ordóñez Torres, K., \& Tarasco, M. M. (2018). [Portuguese] Retracción de artículos biomédicos y sus implicaciones éticas [Retraction of biomedical articles and its ethical implications]. Revista Latinoamericana de Bioética, 18(2), 100-125. https://doi.org/10.18359/rlbi.3446

Ortega, J. L. (2020). The relationship and incidence of three editorial notices in PubPeer: Errata, expressions of concern, and retractions. Learned Publishing, 34(2), 164-174. https://doi.org/10.1002/leap.1339

Ozair, A., Bhat, V., \& Omama, M. (2021). Retractions and withdrawals in neurology literature: A 2020 Analysis of the Retraction Watch database (2668). American Academy of Neurology Annual Meeting, Neurology 96(15 Supplement). https://n.neurology.org/content/96/15 Supplement/2668

Palla Ishfaq, A., Sngson, M., \& Thiyagarajan, S. (2020). A comparative analysis of retracted papers in health sciences from China and India. Accountability in Research, 27(7), 401-416. https://doi.org/10.1080/08989621.2020.1754804

Pantziarka, P., \& Meheus, L. (2019). Journal retractions in oncology: A bibliometric study. Future Oncology, 15(31), 3597-3608. https://doi.org/10.2217/fon-2019-0233

Pardo Pardo, J., Harbin, S., \& Welch, V. (2018). Are retracted studies affecting our reviews? Abstracts of the 25th Cochrane Colloquium, 9(Supp 1). https://abstracts.cochrane.org/2018-edinburgh/are-retracted-studies-affecting-ourreviews

Park, B. S. (2020). Making matters of fraud: Sociomaterial technology in the case of Hwang and Schatten. History of Science, 58(4), 393-416. https://doi.org/10.1177/0073275320921687

Park, J., Lee, J. Y., \& Kwon, O. J. (2018). How the retracted publications are managed and used? A South Korean case. STI 2018 Conference Proceedings, 757-762. https://scholarlypublications.universiteitleiden.nl/handle/1887/65338

Parrish, D. M. (1999). Scientific misconduct and correcting the scientific literature. Academic Medicine: Journal of the Association of American Medical Colleges, 74(3), 221-230. https://doi.org/10.1097/00001888-199903000-00009

Peterson, G. M. (2013). Characteristics of retracted open access biomedical literature: A bibliographic analysis. Journal of the American Society for Information Science and Technology, 64(12), 2428-2436. https://doi.org/10.1002/asi.22944 
Pfeifer, M. P., \& Snodgrass, G. L. (1990). The continued use of retracted, invalid scientific literature. JAMA, 263(10), 1420-1423. https://doi.org/10.1001/jama.1990.03440100140020

Pfeifer, M. P., \& Snodgrass, G. L. (1992). Medical school libraries' handling of articles that report invalid science. Academic Medicine: Journal of the Association of American Medical Colleges, 67(2), 109-113.https://doi.org/10.1097/00001888-199202000$\underline{00014}$

Piller, C. (2021). Disgraced COVID-19 studies are still routinely cited. Science, 371(6527), 331-332. https://doi.org/10.1126/science.371.6527.331

Pirracchio, R., Resche-Rigon, M., Chevret, S., \& Journois, D. (2013). Do simple screening statistical tools help to detect reporting bias? Annals of Intensive Care, 3(1), 29. https://doi.org/10.1186/2110-5820-3-29

Qi, X. S., Deng, H., \& Guo, X. Z. (2017). Characteristics of retractions related to faked peer reviews: An overview. Postgraduate Medical Journal, 93(1102), 499-503. https://doi.org/10.1136/postgradmedj-2016-133969

Qianling, S., Zijun, W., Qi, Z., Ruizhen, H., Xia, G., Shaoe, H., Siya, Z., Yanfang, M., Xianzhuo, Z., Quanlin, G., \& Yaolong, C. (2020). An overview of retraction status and reasons of non-Cochrane systematic reviews in medicine. Research Square. https://doi.org/10.21203/rs.3.rs-125016/v1

Quan-Hoang, V., Viet-Phuong, L., Manh-Tung, H., Thu-Trang, V., \& Manh-Toan, H. (2020). Characteristics of retracted articles based on retraction data from online sources through February 2019. Science Editing, 7(1), 34-44. https://doi.org/10.6087/kcse.187

Rabow, H., \& Rabow, I. (1999). Retraction of articles: Peer-review and quality control. European Conference of Medical and Health Libraries, Libraries without Limits: Changing Needs - Changing Role, 21-22. https://doi.org/10.1007/978-94-011-4621$\underline{04}$

Rada, R. F. (2005). A case study of a retracted systematic review on interactive health communication applications: Impact on media, scientists, and patients. Journal of Medical Internet Research, 7(2), e21.https://doi.org/10.2196/jmir.7.2.e18

Rada, R. F. (2007). Retractions, press releases and newspaper coverage. Health Information and Libraries Journal, 24(3), 210-215. https://doi.org/10.1111/j.14711842.2007.00724.x

Rai, R., \& Sabharwal, S. (2017). Retracted publications in orthopaedics: Prevalence, characteristics, and trends. The Journal of Bone and Joint Surgery. American Volume, 99(9), e44. https://doi.org/10.2106/JBJS.16.01116

Rapani, A., Lombardi, T., Berton, F., del Lupo, V., di Lenarda, R., \& Stacchi, C. (2020). Retracted publications and their citation in dental literature: A systematic review. Clinical and Experimental Dental Research, 6(4), 383-390. https://doi.org/10.1002/cre2.292

Rathmann, J. M. K., \& Rauhut, H. (2019). Teams prevent misconduct: A study of retracted articles from the Web of Science. International Conference on Scientometrics \& Informetrics, Vol. I, 1032-1037. https://www.issi- 
society.org/proceedings/issi 2019/ISSI\%202019\%20-

\%20Proceedings\%20VOLUME\%20I.pdf

Redman, B. K., Yarandi, H. N., \& Merz, J. F. (2008). Empirical developments in retraction. Journal of Medical Ethics, 34(11), 807-809. https://doi.org/10.1136/jme.2007.023069

Resnik, D. B., \& Dinse, G. E. (2013). Scientific retractions and corrections related to misconduct findings. Journal of Medical Ethics, 39(1), 46-50. https://doi.org/10.1136/medethics-2012-100766

Resnik, D. B., Wager, E., \& Kissling, G. E. (2015). Retraction policies of top scientific journals ranked by impact factor. Journal of the Medical Library Association, 103(3), 136-139. https://doi.org/10.3163/1536-5050.103.3.006

Ribeiro, M. D., \& Vasconcelos, S. M. R. (2018). Retractions covered by Retraction Watch in the 2013-2015 period: Prevalence for the most productive countries. Scientometrics, 114(2), 719-734. https://doi.org/10.1007/s11192-017-2621-6 (Correction published January 31, 2018, Scientometrics, 114, p. 735, https://doi.org/10.1007/s11192-018$\underline{2653-6)}$

Roe, P., \& Lewison, G. (2019). The anatomy of retracted papers in the Web of Science, 1998-2017. International Conference on Scientometrics \& Informetrics, Vol. I, 59-64. https://www.issi-society.org/proceedings/issi 2019/ISSI\%202019\%20\%20Proceedings\%20VOLUME\%20l.pdf

Rosenkrantz, A. B. (2016). Retracted publications within radiology journals. American Journal of Roentgenology, 206(2), 231-235. https://doi.org/10.2214/AJR.15.15163 (Correction published April 2016, American Journal of Roentgenology, 206(4), p. 901, https://doi.org/10.2214/AJR.16.16250)

Rossouw, T. M., Matsau, L., \& van Zyl, C. (2020). An analysis of retracted articles with authors or co-authors from the African region: Possible implications for training and awareness raising. Journal of Empirical Research on Human Research Ethics, 15(5), 478-493. https://doi.org/10.1177/1556264620955110

Rubbo, P. (2018). [Portuguese] A Desonestidade Científica e seu Reflexo na Retratação de Artigos Indexados na Base Web of Science, no Período de 1945 a 2015 [Scientific Dishonesty and its Impact on the Retraction of Articles Indexed in the Web of Science Database, from 1945 to 2015] [Doctoral dissertation, Universidade Tecnológica Federal do Paraná]. http://repositorio.utfpr.edu.br/jspui/handle/1/3552

Rubbo, P., Alberto Pilatti, L., \& Picinin, C. T. (2019). Citation of retracted articles in engineering: A study of the Web of Science database. Ethics \& Behavior, 29(8), 661679. https://doi.org/10.1080/10508422.2018.1559064

Rubbo, P., Helmann, C. L., dos Santos, C. B., \& Pilatti, L. A. (2019). Retractions in the engineering field: A study on the Web of Science database. Ethics \& Behavior, 29(2), 141-155. https://doi.org/10.1080/10508422.2017.1390667

Ruy, T. (2014). A need to archive correct biomedical scientific data and to prevent continued citation of retracted scientific publications. Retrospect and Prospect: Essays from the 2013 Taiwan e-Learning and Digital Archives Program International Conference, 8(Supplement), 29-37. https://doi.org/10.3366/ijhac.2014.0097 
Saikia, P., \& Thakuria, B.. (2019). Retraction of papers authored by Yuhji Saitoh-Beyond the Fujii phenomenon. Indian Journal of Anaesthesia, 63(7), 571-584.

https://doi.org/10.4103/ija.IJA 26719

Saiz, L. C., Erviti, J., Garjón, J., Elizondo, J., Azparren, A., Gaminde, I., \& Áriz, M. J. (2016). Cochrane Reviews as privileged sources to report misconduct behaviours: An informative case of duplicate publication. Abstracts of the 24th Cochrane Colloquium, 166. https://abstracts.cochrane.org/2016-seoul/cochrane-reviews-privileged-sourcesreport-misconduct-behaviours-informative-case

Samp, J. C., Schumock, G. T., \& Pickard, A. S. (2012). Retracted publications in the drug literature. Pharmacotherapy, 32(7), 586-595. https://doi.org/10.1002/j.18759114.2012.01100.x

Samuel, S., Cherian, J. M., \& Thomas, A. M. (2020). Comprehensive analysis of retracted publications in dentistry: A 23-year review. International Journal of Dentistry, 2020, 8881352. https://doi.org/10.1155/2020/8881352

Santos-d'Amorim, K., de Melo, R. R., \& dos Santos, R. N. M. (2021). Retractions and postretraction citations in the COVID-19 infodemic: Is academia spreading misinformation? [Retratações e citações pós-retratação na infodemia de COVID-19: A Academia está espalhando desinformação?]. Liinc Em Revista, 17(1), e5593-e5593.

https://doi.org/10.18617/liinc.v17i1.5593

Sarathchandra, D., \& McCright, A. M. (2017). The effects of media coverage of scientific retractions on risk perceptions. SAGE Open, 7(2).

https://doi.org/10.1177/2158244017709324

Sawano, T., Ozaki, A., Saito, H., Shimada, Y., \& Tanimoto, T. (2019). Payments from pharmaceutical companies to authors involved in the valsartan scandal in Japan. JAMA Network Open, 2(5), e193817.

https://doi.org/10.1001/jamanetworkopen.2019.3817 (Correction published July 19, 2019, JAMA Network Open, 2(7), p. e198619, https://doi.org/10.1001/jamanetworkopen.2019.8619)

Schmidt, M. (2018). An analysis of the validity of retraction annotation in PubMed and the Web of Science. Journal of the American Society for Information Science and Technology, 69(2), 318-328. https://doi.org/10.1002/asi.23913

Schneider, J., Ye, D., Hill, A. M., \& Whitehorn, A. S. (2020). Continued post-retraction citation of a fraudulent clinical trial report, 11 years after it was retracted for falsifying data. Scientometrics, 125(3), 2877-2913. https://doi.org/10.1007/s11192-020-03631-1

Schubotz, M., Teschke, O., Stange, V., Meuschke, N., \& Gipp, B. (2019). Forms of plagiarism in digital mathematical libraries. Intelligent Computer Mathematics, LNCS 11617, 258-274. https://doi.org/10.1007/978-3-030-23250-4 18

scite. (2020). Which research journals cite the most retracted work? [Blog post]. Medium. https://medium.com/scite/which-research-journals-cite-the-most-retracted-work9d256dc433ed

Seife, C. (2015). Research misconduct identified by the US Food and Drug Administration: Out of sight, out of mind, out of the peer-reviewed literature. JAMA Internal Medicine, 175(4), 567-577. https://doi.org/10.1001/jamainternmed.2014.7774 
Serghiou, S., Marton, R. M., \& loannidis, J. P. A. (2021). Media and social media attention to retracted articles according to Altmetric. PLOS ONE, 16(5), e0248625.

https://doi.org/10.1371/journal.pone.0248625

Shah, K., Charan, J., Sinha, A., \& Saxena, D. (2021). Retraction rates of research articles addressing COVID-19 pandemic: Is it the evolving COVID epidemiology or scientific misconduct? Indian Journal of Community Medicine, 46(2), 352-354.

https://doi.org/10.4103/ijcm.IJCM 73220

Shah, T. A., Gul, S., Bashir, S., Ahmad, S., Huertas, A., Oliveira, A., Gulzar, F., Najar, A. H., \& Chakraborty, K. (2021). Influence of accessibility (open and toll-based) of scholarly publications on retractions. Scientometrics, 126, 4589-4606.

https://doi.org/10.1007/s11192-021-03990-3 (Correction published May 19, 2021, Scientometrics, 126, p. 4607, https://doi.org/10.1007/s11192-021-04041-7)

Shamim, T. (2018). Data regarding articles retracted from PubMed indexed dental journals from India. Data in Brief, 18, 1069-1072. https://doi.org/10.1016/j.dib.2018.03.133

Sharma, K. (2020). Patterns of retractions from 1981-2020: Does a fraud lead to another fraud? arXiv. http://arxiv.org/abs/2011.13091

Shema, H., Hahn, O., Mazarakis, A., \& Peters, I. (2019). Retractions from altmetric and bibliometric perspectives. Information - Wissenschaft Und Praxis, 70(2-3), 98-110. https://doi.org/10.1515/iwp-2019-2006

Shuai, X., Rollins, J., Moulinier, I., Custis, T., Edmunds, M., \& Schilder, F. (2017). A multidimensional investigation of the effects of publication retraction on scholarly impact. Journal of the Association for Information Science and Technology, 68(9), 2225-2236. https://doi.org/10.1002/asi.23826

Singh, H. P., Mahendra, A., Yadav, B., Singh, H., Arora, N., \& Arora, M. (2014). A comprehensive analysis of articles retracted between 2004 and 2013 from biomedical literature-A call for reforms. Journal of Traditional and Complementary Medicine, 4(3), 136-139. https://doi.org/10.4103/2225-4110.136264

Snodgrass, G. L., \& Pfeifer, M. P. (1992). The characteristics of medical retraction notices. Bulletin of the Medical Library Association, 80(4), 328-334.

https://www.ncbi.nlm.nih.gov/pmc/articles/PMC225694/

Soltani P \& Patini R. (2020). Retracted COVID-19 articles: A side-effect of the hot race to publication. Scientometrics, 125, 819-822. https://doi.org/10.1007/s11192-020-03661$\underline{9}$

Sotudeh, H., Barahmand, N., Yousefi, Z., \& Yaghtin, M. (2020). How do academia and society react to erroneous or deceitful claims? The case of retracted articles' recognition [Online First]. Journal of Information Science. https://doi.org/10.1177/0165551520945853

Souder, L. (2010). A rhetorical analysis of apologies for scientific misconduct: Do they really mean it? Science and Engineering Ethics, 16, 175-184.

https://doi.org/10.1007/s11948-009-9149-y

Springer, A., Chachere, D., Alsarkhi, A., \& Zozus, M. (2020). The impact of information quality on retracted bioinformatics literature. 30th Medical Informatics Europe Conference, 270, 1203-1204. https://doi.org/10.3233/SHTI200363 
Stavale, R., Ferreira, G. I., Galvão, J. A. M., Zicker, F., Novaes, M. R..C..G., de Oliveira, C. M., \& Guilhem, D. (2019). Research misconduct in health and life sciences research: A systematic review of retracted literature from Brazilian institutions. PLoS ONE, 14(4), e0214272. https://doi.org/10.1371/journal.pone.0214272

Steen, R. G. (2011a). Retractions in the medical literature: Who is responsible for scientific integrity? AMWA Journal: American Medical Writers Association Journal, 26(1), 2-7. https://www.amwa.org/resource/resmgr/journal/lssues/2011/2011v26n1 online.pdf

Steen, R. G. (2011b). Retractions in the scientific literature: Do authors deliberately commit research fraud? Journal of Medical Ethics, 37(2), 113-117. https://doi.org/10.1136/jme.2010.038125

Steen, R. G. (2011c). Retractions in the scientific literature: Is the incidence of research fraud increasing? Journal of Medical Ethics, 37(4), 249-253. https://doi.org/10.1136/jme.2010.040923

Steen, R. G. (2011d). Retractions in the medical literature: How many patients are put at risk by flawed research? Journal of Medical Ethics, 37(11), 688-692. https://doi.org/10.1136/ime.2011.043133

Steen, R. G. (2012). Retractions in the medical literature: How can patients be protected from risk? Journal of Medical Ethics, 38(4), 228-232.

https://doi.org/10.1136/medethics-2011-100184

Steen, R. G., Casadevall, A., \& Fang, F. C. (2013). Why has the number of scientific retractions increased? PLoS ONE, 8(7), e68397. https://doi.org/10.1371/journal.pone.0068397 (Correction published July 19, 2013, PLOS ONE, 8(7), https://doi.org/10.1371/annotation/0d28db18-e117-4804-b1bce2da285103ac)

Steen, R. G., \& Hamer, R. M. (2014). A case-control comparison of retracted and nonretracted clinical trials: Can retraction be predicted? Publications, 2(1), 27-37. https://doi.org/10.3390/publications2010027

Stern, A. M., Casadevall, A., Steen, R. G., \& Fang, F. C. (2014). Financial costs and personal consequences of research misconduct resulting in retracted publications. ELife, 3, e02956. https://doi.org/10.7554/eLife.02956

Stojanovski, J. (2015). Do Croatian open access journals support ethical research? Content analysis of instructions to authors. Biochemia Medica, 25(1), 12-21. https://doi.org/10.11613/BM.2015.002

Stretton, S., Bramich, N. J., Keys, J. R., Monk, J. A., Ely, J. A., Haley, C., Woolley, M., \& Woolley, K. L. (2012). Publication misconduct and plagiarism retractions: A systematic, retrospective study. Current Medical Research and Opinion, 28(10), 1575-1583. https://doi.org/10.1185/03007995.2012.728131

Stricker, J., \& Guenther, A. (2019). Scientific misconduct in psychology: A systematic review of prevalence estimates and new empirical data. Zeitschrift Für Psychologie, 227(1), 53-63. https://doi.org/10.1027/2151-2604/a000356

Suelzer, E. M., Deal, J., Hanus, K. L., Ruggeri, B., Sieracki, R., \& Witkowski, E. (2019). Assessment of citations of the retracted article by Wakefield et al with fraudulent claims 
of an association between vaccination and autism. JAMA Network Open, 2(11), e1915552. https://doi.org/10.1001/jamanetworkopen.2019.15552

Suelzer, E. M., Deal, J., Hanus, K. L., Ruggeri, B., \& Witkowski, E. (2020). Identifying barriers to citing retracted literature. Medical Library Association Annual Meeting. Medical Library Association Annual Meeting. https://drive.google.com/file/d/1qKoPEj UAYlw7WPRO8yLAq9UfB0JnfYA/view?usp=s haring

Sugawara, Y., Tanimoto, T., Miyagawa, S., Murakami, M., Tsuya, A., Tanaka, A., Kami, M., \& Narimatsu, H. (2017). Scientific misconduct and social media: Role of Twitter in the stimulus triggered acquisition of pluripotency cells scandal. Journal of Medical Internet Research, 19(2), e57. https://doi.org/10.2196/jmir.6706

Tang, L., Hu, G., Sui, Y., Yang, Y., \& Cao, C. (2020). Retraction: The "other face" of research collaboration? Science and Engineering Ethics, 26, 1681-1708. https://doi.org/10.1007/s11948-020-00209-1

Tao, W., Yong-Hong, L., \& Xing, Q. R. (2020). Characteristics of retracted systematic reviews and meta-analyses in the biomedical literature. Research Square. https://doi.org/10.21203/rs.3.rs-45086/v1

Teixeira da Silva, J. A., \& Dobránszki, J. (2017). Highly cited retracted papers. Scientometrics, 110(3), 1653-1661. https://doi.org/10.1007/s11192-016-2227-4

Teixeira da Silva, J. A., \& Dobránszki, J. (2017). Notices and policies for retractions, expressions of concern, errata and corrigenda: Their importance, content, and context. Science and Engineering Ethics, 23(2), 521-554. https://doi.org/10.1007/s11948-0169769-y

Teixeira da Silva, J. A., \& Erfanmanesh, M. (2021). Errata and retractions associated with research papers published by authors with Hungarian affiliations. European Science Editing, 47, e60203. https://doi.org/10.3897/ese.2021.e60203

Theis-Mahon, N., \& Bakker, C. J. (2020). The continued citation of retracted publications in dentistry. Journal of the Medical Library Association, 108(3), 389-397. https://doi.org/10.5195/jmla.2020.824

Tolsgaard, M. G., Ellaway, R., Woods, N., \& Norman, G. (2019). Salami-slicing and plagiarism: How should we respond? Advances in Health Sciences Education, 24(1), 3-14. https://doi.org/10.1007/s10459-019-09876-7

Tourish, D. (2019). Paradise lost but not regained: Retractions and management studies. In Management Studies in Crisis: Fraud, Deception and Meaningless Research (pp. 106132). Cambridge University Press. https://doi.org/10.1017/9781108616669.006

Tourish, D., \& Craig, R. (2020). Research misconduct in business and management studies: Causes, consequences, and possible remedies. Journal of Management Inquiry, 29(2), 174-187. https://doi.org/10.1177/1056492618792621

Trikalinos, N. A., Evangelou, E., \& loannidis, J. P. A. (2008). Falsified papers in high-impact journals were slow to retract and indistinguishable from nonfraudulent papers. Journal of Clinical Epidemiology, 61(5), 464-470. https://doi.org/10.1016/j.jclinepi.2007.11.019 
Tripathi, M., Dwivedi, G., Sonkar, S. K., \& Kumar, S. (2018). Analysing retraction notices of scholarly journals: A study. DESIDOC Journal of Library \& Information Technology, 38(5), 305-311. https://doi.org/10.14429/djlit.38.5.13103

Tripathi, M., Sonkar, S. K., \& Kumar, S. (2019). A cross sectional study of retraction notices of scholarly journals of science. DESIDOC Journal of Library \& Information Technology, 39(2), 74-81. https://doi.org/10.14429/djlit.39.2.14000

Urquhart, B., MacLehose, H., \& Foxlee, R. (2017). A database to record the impact of fraud and misconduct in studies included in systematic reviews. Abstracts of the Global Evidence Summit, 9(Supp 1), 101. https://abstracts.cochrane.org/2017-globalevidence-summit/database-record-impact-fraud-and-misconduct-studies-includedsystematic

van der Vet, P. E., \& Nijveen, H. (2016). Propagation of errors in citation networks: A study involving the entire citation network of a widely cited paper published in, and later retracted from, the journal Nature. Research Integrity and Peer Review, 1, 3. https://doi.org/10.1186/s41073-016-0008-5

van der Walt, W., Willems, K. A., Friedrich, W., Hatsu, S., \& Krauss, K. (2020). Retracted COVID-19 papers and the level of "citation pollution": A preliminary analysis and directions for future research. Cahiers de La Documentation - Bladen Voor Documentatie, 3-4, 206-218. http://hdl.handle.net/10962/167732

van Leeuwen, T. (2019). Analysis of retracted papers: Initiators and reasons for retraction [proxy for paper]. In COPE Seminar. https://publicationethics.org/resources/seminarsand-webinars/european-seminar-2019-analysis-retractions-initiators-and-reasons

van Leeuwen, T., \& Luwel, M. (2014). An in-depth analysis of papers retracted in the Web of Science. Proceedings of the 19th International Conference on Science and Technology Indicators, 337-344. https://www.researchgate.net/profile/TindaroCicero/publication/265396216 Research quality characteristics of publications and socio-

demographic features of Universities and Researchers evidence from the Italian VQR 2004-2010 evaluation exercise/links/540d89180cf2df04e754b658/Researchquality-characteristics-of-publications-and-socio-demographic-features-of-Universitiesand-Researchers-evidence-from-the-Italian-VQR-2004-2010-evaluationexercise.pdf\#page $=349$

van Noorden, R. (2011). Science publishing: The trouble with retractions. Nature, 478(7367), 26-28. https://doi.org/10.1038/478026a

Vaughan, L., Glanzel, W., Korch, C., \& Capes-Davis, A. (2017). Widespread use of misidentified cell line KB (HeLa): Incorrect attribution and its impact revealed through mining the scientific literature. Cancer Research, 77(11), 2784-2788. https://doi.org/10.1158/0008-5472.CAN-16-2258

Vaught, M., Jordan, D. C., \& Bastian, H. (2017). Concern noted: A descriptive study of editorial expressions of concern in PubMed and PubMed Central. Research Integrity and Peer Review, 2, 10. https://doi.org/10.1186/s41073-017-0030-2

Vuong, Q. H. (2019a). Retraction data can bring more insights and implications for not just authors and their institutions, but funders, policy-makers, and editors. SSRN.

https://doi.org/10.2139/ssrn.3427713 
Vuong, Q. H. (2019b). The retraction lessons that may require academic publishing to change. SSRN. https://doi.org/10.2139/ssrn.3427792

Vuong, Q. H. (2020). The limitations of retraction notices and the heroic acts of authors who correct the scholarly record: An analysis of retractions of papers published from 1975 to 2019. Learned Publishing, 33(2), 119-130. https://doi.org/10.1002/leap.1282

Wager, E., \& Williams, P. (2011). Why and how do journals retract articles? An analysis of Medline retractions 1988-2008. Journal of Medical Ethics, 37(9), 567-570. https://doi.org/10.1136/jme.2010.040964

Walsh, J. P., Lee, Y. N., \& Tang, L. (2019). Pathogenic organization in science: Division of labor and retractions. Research Policy, 48(2), 444-461. https://doi.org/10.1016/j.respol.2018.09.004

Wang, B., Lai, J., Yan, X., Jin, F., \& Yao, C. (2020). Exploring the characteristics, global distribution and reasons for retraction of published articles involving human research participants: A literature survey. European Journal of Internal Medicine, 78, 145-146. https://doi.org/10.1016/j.ejim.2020.03.016

Wang, J., Ku, J. C., Alotaibi, N. M., \& Rutka, J. T. (2017). Retraction of neurosurgical publications: A systematic review. World Neurosurgery, 103, 809-814.e1. https://doi.org/10.1016/j.wneu.2017.04.014

Wang, T., Xing, Q. R., Wang, H., \& Chen, W. (2019). Retracted publications in the biomedical literature from open access journals. Science and Engineering Ethics, 25, 855-868. https://doi.org/10.1007/s11948-018-0040-6

Wang, T., \& Xing, Q. R. (2020). Retracted publications in the biomedical literature from 2012-2018: An overview. Research Square. https://doi.org/10.21203/rs.2.23808/v1

Wartolowska, K., Irving, F., DeVito, N., Feakins, B., Curtis, H., Bacon, S., Smeeth, L., Heneghan, C., \& Goldacre, B. (2019). RetractoBot: A protocol for a randomised controlled trial to assess the impact of notifying authors that they have cited a retracted paper [Registered Report Stage 1 Protocol]. [FigShare Protocol]. https://doi.org/10.6084/m9.figshare.7731665.v1

Wasiak, J., Hamilton, D. G., Foroudi, F., \& Faggion, C. M. Jr. (2018). Surveying retracted studies and notices within the field of radiation oncology. International Journal of Radiation Oncology, Biology, Physics, 102(3), 660-665.

https://doi.org/10.1016/j.jijrobp.2018.06.028

Whitely, W. P., Rennie, D., \& Hafner, A. W. (1994). The scientific community's response to evidence of fraudulent publication: The Robert Slutsky case. JAMA, 272(2), 170-173. https://doi.org/10.1001/jama.1994.03520020096029

Wiedermann, C. J. (2018a). Inaction over retractions of identified fraudulent publications: Ongoing weakness in the system of scientific self-correction. Accountability in Research, 25(4), 239-253. https://doi.org/10.1080/08989621.2018.1450143

Wiedermann, C. J. (2018b). Undisclosed conflicts of interest in German-language textbooks of anesthesiology, critical care, and emergency medicine. Zeitschrift Für Evidenz, Fortbildung Und Qualität Im Gesundheitswesen, 139, 53-58.

https://doi.org/10.1016/j.zefq.2018.10.004 
Wiedermann, C. J., \& Joannidis, M. (2018). The Boldt scandal still in need of action: The example of colloids 10 years after initial suspicion of fraud. Intensive Care Medicine, 44(10), 1735-1737. https://doi.org/10.1007/s00134-018-5289-3 (Correction published October 13, 2020, Intensive Care Medicine, 47, p. 264, https://doi.org/10.1007/s00134$\underline{020-06270-5)}$

Williams, P., \& Wager, E. (2013). Exploring why and how journal editors retract articles: Findings from a qualitative study. Science and Engineering Ethics, 19(1), 1-11. https://doi.org/10.1007/s11948-011-9292-0

Woolley, K. L., Lew, R. A., Stretton, S., Ely, J. A., Bramich, N. J., Keys, J. R., Monk, J. A., \& Woolley, M. (2011). Lack of involvement of medical writers and the pharmaceutical industry in publications retracted for misconduct: A systematic, controlled, retrospective study. Current Medical Research and Opinion, 27(6), 1175-1182.

https://doi.org/10.1185/03007995.2011.573546

Wray, K. B., \& Andersen, L. E. (2018). Retractions in Science. Scientometrics, 117(3), 2009-2019. https://doi.org/10.1007/s11192-018-2922-4

Wright, K., \& McDaid, C. (2011). Reporting of article retractions in bibliographic databases and online journals. Journal of the Medical Library Association, 99(2), 164-167. https://doi.org/10.3163/1536-5050.99.2.010

Wright, N. D. (1991). A Citation Context Analysis of Retracted Scientific Articles [Doctoral dissertation, University of Maryland]. ProQuest Dissertations and Theses. https://www.proquest.com/openview/59a30828c20b9791a5b7023a8b4beaca/1?pqorigsite $=$ gscholar $\&$ cbl $=18750 \&$ diss $=y$

Xu, S. B., \& Hu, G. (2020). Retraction notices as a high-stakes academic genre: A move analysis. In Lin Kathy Ling, Mwinlaaru Isaac N, \& Tay Dennis (Eds.), Approaches to Specialized Genres (pp. 101-120). Routledge. https://doi.org/10.4324/9780429053351-8

Xu, S. B., \& Hu, G. (2018). Retraction Notices: Who authored them? Publications, 6(1), 2. https://doi.org/10.3390/publications6010002

Yan, J., MacDonald, A., Baisi, L. P., Evaniew, N., Bhandari, M., \& Ghert, M. (2016). Retractions in orthopaedic research a systematic review. Bone \& Joint Research, 5(6), 263-268. https://doi.org/10.1302/2046-3758.56.BJR-2016-0047

Yang, K., Zhou, Q., \& Chen, Y. (2019). Impact of the retracted non-Cochrane Review on clinical practice guidelines. Abstracts of the 26th Cochrane Colloquium, 1(Supp 1). https://abstracts.cochrane.org/2019-santiago/impact-retracted-non-cochrane-reviewclinical-practice-guidelines

Yang, S., \& Qi, F. (2020). How do retractions influence the citations of retracted articles? International Conference on Asian Digital Libraries, LNCS 12504, 139-148. https://doi.org/10.1007/978-3-030-64452-9 12

Yang, S., Qi, F., \& Diao, H. (2020). Exploring the influence of publication retraction on citations in psychology science. JCDL '20: Proceedings of the ACM/IEEE Joint Conference on Digital Libraries in 2020, 501-502.

https://doi.org/10.1145/3383583.3398583 
Yeo-Teh, N. S. L., \& Tang, B. L. (2021). An alarming retraction rate for scientific publications on Coronavirus Disease 2019 (COVID-19). Accountability in Research, 28(1), 47-53. https://doi.org/10.1080/08989621.2020.1782203

Zegler-Poleska, E. (2020). 'This paper has been withdrawn.' An empirical analysis of arXiv withdrawals. 4S Annual Meeting. 4S Annual Meeting.

https://convention2.allacademic.com/one/ssss/ssss20/index.php?program focus=view paper\&selected paper $\mathrm{id}=1659648 \& \mathrm{cmd}=$ online program direct link\&sub action $=0$ nline program\#selected tag

Zhang, C., Ding, K., \& Liu, Z. (2019). Informetric analysis on the international retracted publication based on the Web of Science database. International Conference on Social Science and Contemporary Humanity Development, 376, 576-585. https://doi.org/10.2991/sschd-19.2019.100

Zhang, M., \& Grieneisen, M. L. (2013). The impact of misconduct on the published medical and non-medical literature, and the news media. Scientometrics, 96(2), 573-587. https://doi.org/10.1007/s11192-012-0920-5

Zhang, Q., Abraham, J., \& Fu, H. Z. (2020). Collaboration and its influence on retraction based on retracted publications during 1978-2017. Scientometrics, 125(1), 213-232. https://doi.org/10.1007/s11192-020-03636-w

Zhao, T., Dai, T., Lun, Z., \& Gao, Y. (2021). An analysis of recently retracted articles by authors affiliated with hospitals in mainland China. Journal of Scholarly Publishing, 52(2), 107-122. https://doi.org/10.3138/jsp.52.2.03

Zuckerman, H. (2020). Is "the time ripe" for quantitative research on misconduct in science? Quantitative Science Studies, 1(3), 945-958. https://doi.org/10.1162/qss a 00065 (Erratum published December 1, 2020, Quantitative Science Studies, 1(4), p. 1653, https://doi.org/10.1162/qss e 00094)

\section{Associated Data Sets}

Randi Proescholdt, Jodi Schneider \& RISRS Team (2020-1). RISRS Bibliography v.1 https://github.com/infoqualitylab/EPPI-Reviewer to Exhibit JSON/releases/tag/v.1.0.0

Randi Proescholdt, Yoss Arianlou, Katherine Howell, Jodi Schneider \& RISRS Team (2021). RISRS Bibliography v.2 https://github.com/infoqualitylab/EPPI-

Reviewer to Exhibit JSON/releases/tag/2.0.0

Randi Proescholdt, Yoss Arianlou, Katherine Howell, Jodi Schneider \& RISRS Team (2021). RISRS Bibliography v.2.1 https://github.com/infoqualitylab/EPPI-

Reviewer to Exhibit JSON/releases/tag/2.1.0

Randi Proescholdt, Yoss Arianlou, Katherine Howell, Jodi Schneider \& RISRS Team (2021). RISRS Bibliography v.2.15 https://github.com/infoqualitylab/EPPI-

Reviewer to Exhibit JSON/releases/tag/2.15

Proescholdt, Randi (2021): RISRS Retraction Review - Field Variation Data. University of Illinois at Urbana-Champaign. https://doi.org/10.13012/B2IDB-2070560 V1 


\section{CRediT Contributor Statement for Appendix C: Literature Scoping Review}

Methods and Intermediate Results

Yoss Arianlou: Data curation, Investigation; Halle Burns: Data curation, Investigation; Yuanxi Fu: Data curation, Investigation; Katherine Howell: Data curation, Investigation, Writing review \& editing; Tzu-Kun (Esther) Hsiao: Software; Linxi Liu: Conceptualization, Data curation, Investigation, Methodology, Software; Pamela Nila: Data curation, Investigation; Randi Proescholdt: Conceptualization, Data curation, Investigation, Methodology, Project administration, Software, Validation, Writing - original draft, Writing - review \& editing; M. Janina Sarol: Conceptualization, Data curation, Investigation, Methodology, Software; Jodi Schneider: Conceptualization, Data curation, Funding acquisition, Investigation, Methodology, Project administration, Supervision; Nathan D. Woods: Data curation, Investigation; Yee Yan

'Vivien' Yip: Data curation, Investigation.

\section{BIBLIOGRAPHY}

Budd, J. M., Sievert, M., \& Schultz, T. R. (1998). Phenomena of retraction: Reasons for retraction and citations to the publications. JAMA, 280(3), 296. https://doi.org/10.1001/jama.280.3.296

Chen, C., Hu, Z., Milbank, J., \& Schultz, T. (2013). A visual analytic study of retracted articles in scientific literature. Journal of the American Society for Information Science and Technology, 64(2), 234-253. https://doi.org/10.1002/asi.22755

Grieneisen, M. L., \& Zhang, M. (2012). A comprehensive survey of retracted articles from the scholarly literature. PLoS ONE, 7(10), e44118. https://doi.org/10.1371/journal.pone.0044118

Hesselmann, F., Graf, V., Schmidt, M., \& Reinhart, M. (2017). The visibility of scientific misconduct: A review of the literature on retracted journal articles. Current Sociology. La Sociologie Contemporaine, 65(6), 814-845. https://doi.org/10.1177/0011392116663807

Page, M. J., McKenzie, J. E., Bossuyt, P. M., Boutron, I., Hoffmann, T. C., Mulrow, C. D., Shamseer, L., Tetzlaff, J. M., Akl, E. A., Brennan, S. E., Chou, R., Glanville, J., Grimshaw, J. M., Hróbjartsson, A., Lalu, M. M., Li, T., Loder, E. W., Mayo-Wilson, E., McDonald, S., ... Moher, D. (2021). The PRISMA 2020 statement: An updated guideline for reporting systematic reviews. BMJ, 372, n71. https://doi.org/10.1136/bmj.n71

Pfeifer, M. P., \& Snodgrass, G. L. (1990). The continued use of retracted, invalid scientific literature. The Journal of the American Medical Association, 263(10), 1420-1423. https://doi.org/10.1001/jama.1990.03440100140020

Sarol, M. J., Liu, L., \& Schneider, J. (2018). Testing a citation and text-based framework for retrieving publications for literature reviews. Proceedings of the 7th International Workshop on Bibliometric-Enhanced Information Retrieval (BIR 2018) Co-Located with the 40th European Conference on Information Retrieval (ECIR 2018), 22-33. http://ceurws.org/Vol-2080/paper3.pdf 


\section{Appendix D: Stakeholder Consultation Process}

Nathan D. Woods, M.T. Campbell, Jodi Schneider, and the RISRS Team

To accompany "Recommendations from the Reducing the Inadvertent Spread of Retracted Science: Shaping a Research and Implementation Agenda Project" July 2021

Stakeholders engaged in the RISRS project have contributed to ongoing rounds of feedback, integration and dissemination, with the aim of introducing change into the scientific publishing ecosystem. Stakeholder dialogue and synthesis has been key to deriving a clear understanding of how retracted science is understood in different professional and sector domains. The RISRS team is developing an ecosystem map of concrete actions needed to support cross-sectoral collaboration, which will ultimately help identify pathways for effective implementation.

\section{Stakeholder Consultation}

Stakeholder consultation in the RISRS process plays a central role in identifying problems, possible solutions, and collaborative implementation strategies. Stakeholders were invited to engage with the project starting in July 2020. All stakeholders were invited on the basis of their professional expertise and roles in publishing, research, information technology, university and government. Stakeholders included funders, editors, peer reviewers/authors based at both universities and government research facilities, commercial and scholarly publishers, individual researchers, librarians, platform and database providers, university research integrity officers, science journalists, staff at professional organizations, and members of standards setting organizations.

Stakeholders were invited to participate in the RISRS project in multiple ways, and were given the choice to participate in an hour-long interview, to contribute an original position paper, to be included on the project website, and to participate in an online workshop series. These contributions were subsequently used in a variety of synthesis activities, including the design of the workshop, and the resulting white paper recommendations. Informed consent materials were subsequently sent to those who agreed to participate in an interview or attend the workshop.

\section{Stakeholder Invitation Process}

Stakeholder invitations were organized in three rounds, beginning with the formation of an advisory group starting in March 2019. Additional stakeholders were subsequently invited to participate starting in July 2020, following funding from the Alfred P. Sloan Foundation and Institutional Research Board approval, and adjustments to the workshop schedule as it moved 
online. All stakeholders were invited to participate via an introductory email that included information on the general goals of the project and a link to the project website.

In the first round, stakeholders were invited on the basis of professional contacts, recommendations from the advisory board, and proposal review comments from the Alfred $P$. Sloan Foundation. The second round of targeted invitations were based on both advisory board recommendation, consulting available professional networks, and identifying people at relevant organizations via Linkedln and organizational websites. Finally, in the third stage, invitees were selected through peer-nomination (snowball sampling).

Invitees were asked to participate broadly in the RISRS process, either through an interview, or participation in the workshop, or through the contribution of an original position paper (see Appendix: Workshop Invitation).

\section{Advisory Board}

The stakeholder invitation process is anchored in the role of an advisory board composed of leaders in the field of scholarly communication (see Table 5).

Table 5: RISRS Advisory Board Members by Role

Journal Editor: Annette Flanagin, Executive Managing Editor and Vice President, Editorial Operations, JAMA and The JAMA Network

Research Integrity Officer: C.K. (Tina) Gunsalus, J.D., Director, National Center for Professional \& Research Ethics, University of Illinois at Urbana-Champaign.

Researcher: Daniele Fanelli, PhD, Fellow in Quantitative Methodology, Department of Methodology, London School of Economics and Political Science.

Journalist: Ivan Oransky, MD. Co-Founder of Retraction Watch \& Editor in Chief of Spectrum

The advisory board helped identify and attract stakeholders from across diverse fields related to scholarly communication in several ways. First, the advisory board was tasked with helping to identify stakeholders who might participate in the project workshop, in the interview process, as well as those who might be consulted for specific or ongoing feedback. Second, as recognized leaders in their professional fields, board members have provided trusted feedback from different sectors in the scientific publishing ecosystem. Finally, the advisory board ensured that people with a diversity of perspectives were invited to participate.

\section{Stakeholder Diversity}

Broad inclusivity measures were built into the invitation of potential participants. Diversity of personal and professional experience was a key component of the advisory board selection process. Gender balance - two of the four advisory board members are women, and includes 
one mid-career and one senior professional—was a foundational aspect of this consideration. Additionally, the board was asked to consider further diverse inclusion criteria for enrollment. Our goal was to to include professionals from the global south, women, non-white, as well as women as potential stakeholders, and people from various career stages in their recommendations.

The project committed to ensuring that about half of our participants are women, and during the invitation process we attempted to send $50 \%$ of our invitations to women. In striving for racial and gender diversity in the project, we sought diverse leadership in scholarly communication by consulting members of a diversity of organizations, including:

- The Society for Scholarly Publishing's Diversity and Inclusion Task Force

- Association of Research Libraries: Senior Director of Diversity and Leadership Programs (Mark Puente)

- The Association of American University Presses Equity, Justice \& Inclusion Committee

- The Workplace Equity Project

Invitations to stakeholders were sent in July of 2020 and this process concluded in late October of 2020 with the start of the workshop series. A second round of invitations to participate will begin in February of 2021, and will focus on consulting additional stakeholder roles that were identified as a result of the workshop. Stakeholders consulting in this round of interviews will also be invited to provide feedback on the evolution of the white paper, and implementation model.

\section{Consultative Interviews}

As part of the stakeholder synthesis process, interviews were structured to introduce participants into the project's larger engagement process, as well to consult stakeholders on their ideas, opinions, attitudes and perspectives of retracted research and its continued citation.

Interviewees for the project fall into two broad categories: those who participated in the workshop, and those who only participated in an interview. Interviewees in this second category also included people that could not attend the workshop (e.g. due to timezones), people with special perspectives, such as lawyers working in the publishing industry, or interviewees who had special organizational contexts, but not general knowledge or awareness of issues related to retracted research. Additionally, some stakeholders participated in the workshop, but declined an interview. During the interviews, stakeholders were interviewed on their experience with retracted research, their opinions and attitudes towards the variety of retractions, and the harms associated with retraction, as well as any perspectives or experiences that they might offer as related to retracted research, its continued citation, and the work of maintaining or correcting the scientific record. Interview questions are in a separate section below.

\section{Project Materials}

To disseminate information about the project, the project team created a website providing information about the RISRS objectives and goals, and the names and roles of the RISRS 
research team, advisory board, and workshop participants as they joined. Participantcontributed position papers were added to the website, and placed in IDEALS, an institutional repository maintained by the University of Illinois, Urbana-Champaign. These position papers, the team's preliminary research outputs, and workshop materials were made available to workshop participants in a Google Drive folder, with Word documents available on request.

Materials produced by the RISRS team in preparation for the workshop included: a searchable bibliography derived from the literature review; an initial summary of the literature, focused on continued citation of retracted papers and the quality and visibility of retraction notices; an analysis of metadata errors of retraction status in common databases; results of a thematic analysis of consultations with stakeholders, and a citation analysis. As the project develops, the project website (https://infoqualitylab.org/projects/risrs2020/ ) and the RISRS institutional repository collection (https://www.ideals.illinois.edu/handle/2142/108359 ) will be developed to act as a hub of continued engagement.

\section{Workshop Process and Stakeholder Synthesis}

An intensive three-day stakeholder workshop was built into the design of the RISRS project to facilitate active and engaged dialogue, cross-sector problem solving and collaboration.

\section{Workshop Design for Moving Online}

The proposal called for an in-person workshop to be held over a period of 1.5 days at the Big 10 Conference Center in Chicago. Due to the global pandemic, the proposed in-person workshop was converted to an online format. To preserve the original intention while adapting to the online medium, the workshop was re-designed as a three-part online event.

In transitioning to an online workshop, the RISRS team conducted a landscape scan to understand available platforms, emerging strategies, and potential problems in conducting expert meetings online. Additionally, we consulted with the RISRS advisory board about the feasibility of taking the workshop online. This supported an intensive discussion of scheduling and feedback on how to strategize to overcome divergent timezones, professional schedules and time commitments, and technological limitations. Many of the stakeholders, particularly those coming from government or corporations, faced limitations on which technology platforms they were allowed to use. Similarly, the issue of scheduling proved difficult. Aligning professional schedules across multiple timezones took several rounds of surveys of the advisory board to narrow down to an acceptable time commitment.

\section{Workshop Facilitation}

The design of in-person workshop proposed use of conversational design patterns known as Liberating Structures (Lipmanowicz \& McCandless, 2014)(http://www.liberatingstructures.com). The strength of these patterns is that they allow for distributed control over how topics in meetings are shaped, allowing for the introduction of novel changes to standardized agenda items. Many of the patterns include guided instructions for how groups work together in-person 
for collaborative problem solving and decision making. To translate these strengths into a virtual format, the RISRS team attended multiple online trainings in virtual facilitation.

Ultimately, Liberating Structures shaped our virtual facilitation environment: we aimed for a participatory format that maintained the original intent, using platforms and tools that we could use during the workshop, such as breakout rooms, 'do-athon' formats, and structured plenary sessions. During the first workshop, for example, the opening plenary was organized into a cross-sectoral fishbowl-style discussion organized by professional and industry groupings. By contrast, for the second activity of that day participants were organized around topics of interest derived from preliminary research materials. On subsequent workshop days, participants were organized into working and discussion groups by the RISRS team based on surveys of their preferences, and a selection of topics nominated by workshop participants. This interactive structure allowed stakeholders to offer a diversity of perspectives on a broad number of problems.

Each session utilized a series of collaborative documents designed around conversational scaffolding, providing a focus for problem solving and a series of artifacts that aided in harvesting stakeholder ideas, opinions, and objections. Additionally, members of the RISRS team were embedded in all of the workshop events as notetakers and participant observers, where their notes provided context and detail the construction of these artifacts. These documents, suggestions, and reports, were analyzed by the RISRS team, and the results of the analysis—salient recommendations and themes—were subsequently presented to the stakeholders for refinement, comment or adaptation. Additionally, the RISRS team organized a series of surveys scheduled between workshop meetings to encourage robust feedback on the evolving draft recommendations.

\section{Stakeholder Synthesis}

One advantage of taking the workshop online was that the extended design, with time between workshops, allowed the RISRS team to spend time with the materials produced by stakeholders, and to develop and nuance the organization of the workshop in ways that served to benefit stakeholder collaboration.

As each workshop evolved, participants offered iterative and ongoing feedback, nominating additional areas of interest and functional interest groups that mixed and matched participants over the course of the three workshop sessions. Workshop sessions were organized around a series of synchronous activities that facilitated stakeholder feedback and engagement during the live virtual events. Prior to and between each workshop, the RISRS team combined themes from the interviews and literature reviews to select agenda items for stakeholder discussion. These themes and agenda items were elaborated with structured conversational prompts designed to support stakeholder interaction and cooperative problem-solving. Using these materials, stakeholders discussed common issues, outlined common challenges and unique impediments to action, possible areas of accountability and pathways for implementing actionable change in the future. 
The stakeholder consultation process, and stakeholders' collaborative role in the ongoing project synthesis, help to create a rich, nuanced portrait of the role of retraction in the scientific ecosystem. This portrait is reflected in both the scope of the recommendations and their grounding in actionable industry or sector level concerns. In addition to nuanced conversations about sector-specific retraction issues, this portrait included a number of overlapping leverage points to address the issue of retracted research and its continued citation from multiple complementary angles.

\section{Stakeholder Feedback}

After the second meeting, participants were sent a survey that asked them to evaluate and rank top-level suggestions that had re-occurred throughout the first two meetings. Based on these results, the team generated recommendations for discussion. At meeting three, participants reviewed this "zeroth draft" and refined them into a set of base recommendations, and potential avenues for implementation.

This iterative and ongoing feedback process helped to scope recommendations from broad concerns, to clear possibilities for action. After the workshop, we anticipate a second round of stakeholder consultations to bridge to perspectives that emerged as part of this extended stakeholder consultation, feedback and synthesis dynamic. As further stakeholders are added to the consultation process, their insights will be incorporated into the draft recommendations, and they will be given the opportunity to participate in the stakeholder feedback schedule.

As drafts of the white paper undergo further comment and feedback, these recommendations will be developed into a final body of recommendations, as well as a research agenda and implementation model. Additional viability testing on proposed implementation strategies will undergo an additional feedback and synthesis process involving a smaller body of stakeholder volunteers asked to focus on evaluating the link between recommendations and proposed implementation actions.

\section{Interview Questions}

\section{A. BIOGRAPHY, PROFESSIONAL LIFE AND ROLE}

Q.1. What has been your experience with retracted papers?

Q.2. In your current position have you worked with or encountered papers or articles with a retracted status? Can you describe how you worked with that situation?

\section{B. PROBLEM DEFINITION AND UNDERSTANDING}

Q.3. Please describe the issues involved with retracted papers. How would you characterize the issues?

Q.3.a. In your opinion, what problems do they pose for scientific publishing? 
Q.4. How would you describe the different varieties — types or classes — of retracted papers?

Q.4.a. Are there any of these varieties that can be cited?

Q.4.b What are the conditions for this type of citation?

Q.5. How do you characterize the harm associated with retracted research?

\section{PUBLISHING ECOSYSTEM}

Q.6. Thinking about the publishing industry, how would you describe the process of managing or mitigating the effects of retracted papers?

Q.7. Thinking of this process, who would you say is involved in the process of managing or mitigating the effects of retracted papers?

Q.7.a. What is their role or position?

Q.8. Based on your experience, how would you suggest stopping the spread of retracted papers?

Q.9. Have you had any success collaborating with your colleagues and professional peers to address the spread of retracted papers?

Q.10. In your opinion, would you say there are any special problems associated with Open Access and retracted papers?

Q.10.a Do you see any impediments to retraction statuses and retraction notices for Open Access Publishing?

Q.11. Similarly, in your opinion, would you say there are special problems associated with selfcitation and retracted papers?

Q.11.a. Do you see any impediments to the use of retraction statuses and retraction notices involving self-citation and retracted papers.

\section{Workshop Invitation}

Dr. Jodi Schneider

School of Information Sciences

University of Illinois at Urbana-Champaign $501 \mathrm{E}$. Daniel St

Champaign, IL 61820

$\left[{ }^{* * *}\right.$ Insert name \&address $\left.{ }^{* * *}\right]$ 
Dear $\left[{ }^{* * *}\right.$ name $\left.{ }^{* * *}\right]$, I am pleased to invite you to participate in a virtual workshop entitled "Reducing the Inadvertent Spread of Retracted Science: Shaping a Research and Implementation Agenda." Funded by the Alfred P. Sloan Foundation, this event will bring together a diverse group of stakeholders in the scientific publishing ecosystem to launch a collaborative effort to reduce the citation and reuse of retracted publications.

I am particularly interested in benefiting from your perspective as $\left[{ }^{* * *}\right.$ position $\left.{ }^{* * *}\right]$ and believe you are well positioned to represent the important perspectives of the $\left[{ }^{* * *}{ }^{*}\right.$ ield/industry $\left.{ }^{* * *}\right]$ in the discussion, as well as to communicate the perspectives of other stakeholders back to your peers. The goal of this project is to distill a coherent, actionable set of recommendations for next steps which will be disseminated to a broader community in a white paper and at conferences.

The workshop will be held remotely as a series of structured discussions. During this time, we will have the opportunity to consider the important issue of reducing the continued citation of retracted papers.

In addition to the workshop, your participation may also include:

- A one-hour interview with the project team this spring

- Submission of a 2-4-page discussion paper on this topic by [ ${ }^{* * *}$ date $\left.{ }^{* * *}\right]$.

- Voluntary conversation on discussion papers (identifying problems, suggesting possible reforms, etc.) with other participants prior to the workshop.

To help shape the broader conversation on retracted science, if you choose to participate in the workshop your participation in the workshop, along with your contribution of a discussion paper, and the major outcomes from our work together will be shared on a project website.

If you cannot attend the workshop, please let us know if you would participate in the study interview. Any insights you might share with us would be of value.

We would appreciate your response to this invitation no later than [ ${ }^{* * *}$ date $\left.^{* * *}\right]$. The success of the workshop depends on the expertise and engagement of the stakeholder communities. I would be honored to have you at the table.

Sincerely,

Jodi Schneider (PI)

Assistant Professor

School of Information Sciences

University of Illinois at Urbana-Champaign 
Reducing the Inadvertent Spread of Retracted Science:

Shaping a Research and Implementation Agenda

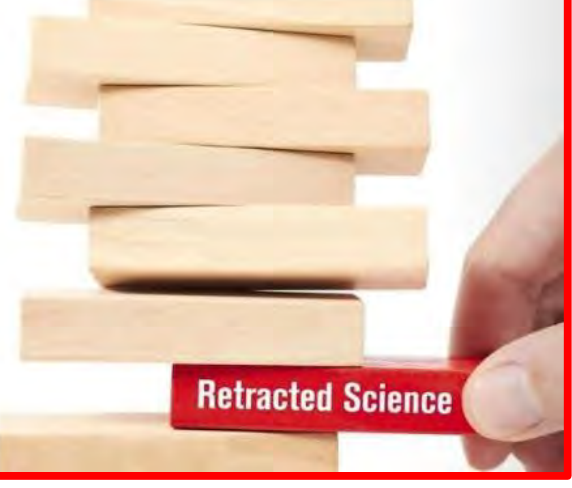

\section{ALFRED P. SLOAN FOUNDATION}

Monday Oct 26th

\section{DAY 1 Listening and Learning}

Welcome and Introductions

6:00-6:20 AM (PT) | 7:00-7:20 AM (MT) | 8:00-8:20 AM (CT) | 9:00-9:20 AM (ET) | 1:00-1:20 PM (UK) | 2:00-2:20 PM (CEST)

\section{Stakeholder Experience Fishbowl Storytelling (20 min per group)}

Structure: 5 min round robin, 10 min discussion, 5 min Q\&A from audience

Based on your experience or expertise, what do you want others to know about retractions? What problems are associated with retraction in your community? Can these problems be solved? How?

GROUP 1 - NORMS, STANDARDS, AND INCENTIVES

6:20-6:40 AM (PT) | 7:20-7:40 AM (MT) | 8:20-8:40 AM (CT) | 9:20-9:40 AM (ET) | 1:20-1:40 PM (UK) | 2:20-2:40 PM (CEST) 
People working in standards organizations, funding agencies, or journalism, or in investigating reform in standards and incentives.

- Monya Baker, Senior Editor, Comment Desk, Nature magazine

- Daniele Fanelli, Fellow in Quantitative Methodology, Department of Methodology, London School of Economics and Political Science

- Ashley Farley, Program Officer, Knowledge \& Research Services, Bill \& Melinda Gates Foundation

- Josh Greenberg, Program Director, Alfred P. Sloan Foundation

- Alice Meadows, Director of Community Engagement, NISO

- Ivan Oransky, Co-Founder of Retraction Watch \& Vice President, Editorial at Medscape, Distinguished Writer In Residence at New York University's Carter Journalism Institute, and president of the Association of Health Care Journalists

- Deborah Poff, Chair of the Trustee Board COPE \& Editor in Chief Journal of Academic Ethics

- Eefke Smit, Director of Standards and Technology, International Association of STM Publishers

\section{GROUP 2 - PUBLISHING INDUSTRY}

6:45-7:05 AM (PT) | 7:45-8:05 AM (MT) | 8:45-9:05 AM (CT) | 9:45-10:05 AM (ET) | 1:45-2:05 PM (UK) | 2:45-3:05 PM (CEST)

People in the publishing industry, e.g. editors, publishers, peer review, managers, quality assurance staff.

- IJsbrand Jan Aalbersberg, Chair of the Standards and Technology Executive Committee of STM; SVP Research Integrity, Elsevier

- Michele Avissar-Whiting, Editor in Chief for Research Square (tentative)

- Annette Flanagin, Executive Managing Editor and Vice President, Editorial Operations, JAMA and The JAMA Network, and Executive Editor, JAMAevidence

- Dan Kulp, Director, Editorial Development for the journals of the American Chemical Society

- Michael Markie, Publishing Director, Life Sciences, F1000

- Katrina Pickersgill, Executive Peer Review Manager, SAGE Publications, Ltd.

- Sarah Robbie, Head of Research Integrity \& Ethics, Taylor and Francis

- Randy Townsend, Director, Journal Operations at American Geophysical Union

\section{GROUP 3 - BRIDGING TECHNOLOGY}




\section{7:10-7:30 AM (PT) | 8:10-8:30 AM (MT) | 9:10-9:30 AM (CT) | 10:10-10:30 AM (ET) | 2:10-2:30 PM (UK) | 3:10-3:30 PM (CEST)}

People whose work supports interlinking and interconnection of scholarly publishing items, e.g. citation verification software, reference management systems, and bibliographic databases (indexing and abstracting, citations) and underlying technologies such as identifiers and metadata.

- Geoff Bilder, Director of Technology and Research, Crossref

- Helena Cousijn, Community Engagement Director, DataCite

- Nicholas DeVito, Doctoral Researcher, EBM DataLab, University of Oxford

- James Leung, Head of Product Management, Kopernio, Clarivate

- Josh Nicholson, Co-founder and CEO scite

- Laura Paglione, Project Lead, Metadata2020

- John Seguin, President and Chief Librarian, Third Iron LLC

- Sean Takats, Director of Zotero \& Professor/Chief Scientist University of Luxembourg

\section{BREAK - 15 MINUTES}

7:30-7:45 AM (PT) | 8:30-8:45 AM (MT) | 9:30-9:45 AM (CT) | 10:30-10:45 AM (ET) | 2:30-2:45 PM (UK) | 3:30-3:45 PM (CEST)

\section{GROUP 4 - RESEARCH INTEGRITY}

7:45-8:05 AM (PT) | 8:45-9:05 AM (MT) | 9:45-10:05 AM (CT) | 10:45-11:05 AM (ET) | 2:45-3:05 PM (UK) | 3:45-4:05 PM (CEST)

People in a research integrity office in government or a university, supporting research integrity efforts/investigations in publishing.

- Elisabeth Bik, Science Integrity consultant \& Microbiome

- Stephanie Boughton, Cochrane Research Integrity Editor

- Francesca Grifo, Scientific Integrity Official Environmental Protection Agency

- CK Gunsalus, Director, National Center for Professional \& Research Ethics, Professor Emerita of Business, and Research Professor at the Coordinated Sciences Laboratory, University of Illinois at Urbana-Champaign

- Christopher Lehmann, Research Integrity Officer, University of Illinois, UrbanaChampaign

\section{GROUP 5 - RESEARCHERS \& LIBRARIANS}




\section{8:10-8:30 AM (PT) | 9:10-9:30 AM (MT) | 10:10-10:30 AM (CT) | 11:10-11:30 AM (ET) | 3:10-3:30 PM (UK) | 4:10-4:30 PM (CEST)}

People producing and/or facilitating access to original research.

- Caitlin Bakker, Medical School Librarian and Research Services Coordinator, Health Sciences Library University of Minnesota

- Jennifer Deal, Librarian Lead and Institutional Repository Manager, Advocate Aurora Library

- Karen Hanus, Director, Advocate Aurora Library, St. Luke's Medical Center

- Tom Heyman, Assistant Professor, Methodology \& Statistics, Faculty of Social Sciences, Leiden University

- Kathryn Kaiser, Assistant Professor, Dept. of Health Behavior, University of Alabama Birmingham

- Kathrin McConnell, Director, FDA Library, Office of Information Management and Technology

- Pamela Ronald, Distinguished Professor, Department of Plant Pathology, College of Agricultural and Environmental Sciences, University of California, Davis

- Barbara Ruggeri, Life \& Health Sciences Librarian, Carroll University

- Elizabeth Suelzer, User Education and Reference Librarian Medical College of Wisconsin

- Nicole Theis-Mahon, Liaison Librarian \& Health Sciences Collection Coordinator, Health Sciences Library University of Minnesota

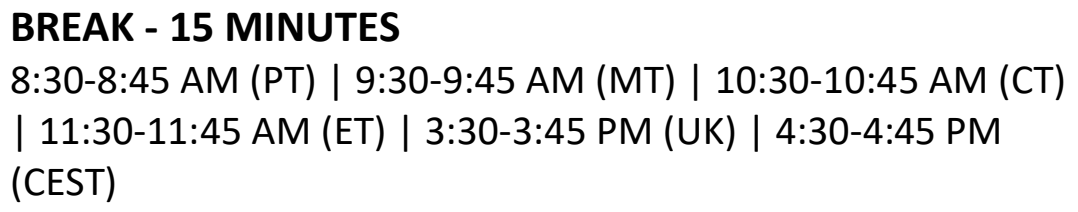

\section{Conversation Café - Thematic Discussions in breakout rooms - 45 min $+\mathbf{5}$ min notetaking 8:50 AM-9:45 (PT) | 9:50-10:45 AM (MT) | 10:50-11:45 AM (CT) | 11:50 AM-12:45 PM (ET) | 3:50-4:45 PM (UK) | 4:50-5:45 PM (CEST)}

Group discussion in 4 rounds:

- First round: Each person shares thoughts about the topic. 5 min

- Second round: Each person shares thoughts after having listened to everybody in the room. $5 \mathrm{~min}$

- Third round: open discussion 25 min

- Fourth round: each member shares takeaways, ideas, and thoughts for next steps. $10 \mathrm{~min}$. 
Group members record takeaways, thoughts, and ideas in shared Google Slides. 5 min.

Participants will self-select a breakout room. See discussion prompts table below.

- Types of retractions

- Informative retraction notices

- Communication of misconduct investigations

- Visibility of retraction status

- Author-initiated retraction

- Rooms 6 - 10: Participant nominated topics TBD

\section{REPORT OUT FROM THEMATIC DISCUSSIONS / GATHERING EMERGING TOPICS FOR} DAY 2 / WRAP-UP

9:45-10 AM (PT) | 10:45-11 AM (MT) | 11:45 AM-noon(CT) | 12:45-1:00 PM (ET) | 4:45-5:00 PM

(UK) | 5:45-6:00 PM (CEST)

\section{TABLE: DISCUSSION PROMPTS FOR CONVERSATION CAFE (Please suggest additional topics!)}

\begin{tabular}{|l|l|}
\hline \multicolumn{1}{|c|}{ Room } & \multicolumn{1}{|c|}{ Prompt } \\
\hline Types of retractions & $\begin{array}{l}\text { What would a common taxonomy for retractions include? What are } \\
\text { industry requirements to make it feasible to apply? }\end{array}$ \\
\hline $\begin{array}{l}\text { Informative retraction } \\
\text { notices }\end{array}$ & $\begin{array}{l}\text { What are the obstacles to including detailed retraction information in } \\
\text { retraction notices such as who is retracting and what is the reason for } \\
\text { the retractions? }\end{array}$ \\
\hline $\begin{array}{l}\text { Communication of } \\
\text { misconduct } \\
\text { investigations }\end{array}$ & $\begin{array}{l}\text { What do publishers need or expect from institutions conducting } \\
\text { misconduct investigations? What are the disincentives for institutions } \\
\text { to collaborate with publishers on issues of retraction? }\end{array}$ \\
\hline $\begin{array}{l}\text { Visibility of retraction } \\
\text { status }\end{array}$ & $\begin{array}{l}\text { What are the obstacles to consistent, clear identification of retraction } \\
\text { status on publisher websites? }\end{array}$ \\
\hline
\end{tabular}




\begin{tabular}{|l|l|}
$\begin{array}{l}\text { Author-initiated } \\
\text { retraction }\end{array}$ & $\begin{array}{l}\text { What options are there for author-initiated retraction? What } \\
\text { challenges are there to author-initiated retractions? }\end{array}$ \\
\hline Funding & What are impacts on funding and funders? \\
\hline $\begin{array}{l}\text { Timeliness in } \\
\text { retractions }\end{array}$ & $\begin{array}{l}\text { What are the primary obstacles to resolving retractions? Are their } \\
\text { ways to speed up or improve the process? }\end{array}$ \\
\hline Action today & Things we can do today to limit the spread of retracted science. \\
\hline $\begin{array}{l}\text { Timeliness of } \\
\text { retractions }\end{array}$ & \\
\hline Room 10 & (please suggest a topic here) \\
\hline
\end{tabular}

Monday November 9th

DAY 2 Collaborative Agenda Setting

\section{Welcome and Review of Agenda \& Logistics (Plenary, $20 \mathrm{~min}$ )}

6:00-6:20 AM (PT) | 7:00-7:20 AM (MT) | 8:00-8:20 AM (CT) | 9:00-9:20 AM (ET) | 2:00-2:20 PM

(UK) | 3:00-3:20 PM (CET)

\section{Implementation Solutions (Breakout group discussions, $\mathbf{3 0} \mathrm{min}$ )}

6:20-6:50AM (PT) | 7:20-7:50 AM (MT) | 8:20-8:50 AM (CT) | 9:20-9:50 AM (ET) | 2:20-2:50 PM (UK) | 3:20-3:50 PM (CET)

STRUCTURE: Each group will have a Google Doc to guide the discussion as follows:

1. List the implementation steps (15 min)

2. Determine who needs to be included (5-7 min)

3. Consider the impediments (5-7 $\mathrm{min})$

TOPICS/GROUPS: 
A. Adoption of COPE guidelines: How do we facilitate further adoption of COPE guidelines as an industry-wide standard?

a. Google Doc Day 2 - A - Adoption of COPE Guidelines

b. Group

i. Yoss Arianlou, University of Illinois Urbana-Champaign

ii. Joerg Heber, Editorial Director PLOS and Editor-in-Chief PLOSOne

iii. Hannah Heckner, Product Strategist, Silverchair

iv. Stacey Lavelle, Senior Business Analyst, Aries Systems Corporation

v. Deborah Poff, Editor in Chief Journal of Academic Ethics, Chair Trustee Board COPE

vi. Sarah Robbie, Head of Research Integrity \& Ethics, Taylor and Francis

vii. Randy Townsend, Director, Journal Operations at American Geophysical Union

\section{B. Use of Retracted Science in Policy, Applied Science and Evidence-Based Practice:}

What actions need to be taken when a retracted item has been used in a regulation, guideline, or systematic review?

a. Google Doc Day 2 - B - Use of Retracted Science in Policy, Applied Science, and Evidence-Based Practice

b. Group

i. Stephanie Boughton, Research Integrity Editor, Cochrane

ii. Stephen Gonsalves, Division of Education and Integrity, US Office of Research Integrity

iii. Francesca Grifo, Scientific Integrity Official, Environmental Protection Agency

iv. Kathryn Kaiser, Assistant Professor Dept of Health Behavior, University of Alabama Birmingham

v. Kathrin Mc Connell, Director, FDA Library, Office of Information Management and Technology

vi. Dmitry Malkov, University of Sussex

vii. Jodi Schneider, University of Illinois Urbana-Champaign

C. Robust Dissemination of Retraction Status: How do we assure robust dissemination of retraction status information? What are the opportunities for sharing information across different scholarly publishing services? What is needed to ensure sustainability and data quality of retraction status information?

a. Google Doc Day 2 - C - Robust Dissemination of Retraction Status

b. Group

i. Ashley Farley, Program Officer, Knowledge \& Research Services, Bill \& Melinda Gates Foundation

ii. Patricia Feeney, Head of Metadata, Crossref

iii. Katie Funk, Program Manager, PubMed Central

iv. James Leung, Product Director, Clarivate Analytics

v. Ivan Oransky, Co-Founder of Retraction Watch 
vi. Randi Proescholdt, University of Illinois at Urbana-Champaign

vii. John Seguin, President and Chief Librarian, Third Iron LLC

D. Retraction Education for Researchers, Editors, and the Public: What does every researcher need to know about retraction? What does every editor need to know? What does the public need to know? Where does this happen and how?

a. Google Doc Day 2 - D - Retraction Education for Researchers, Editors, and the Public

b. Group

i. Elsa Alvaro, Director of Academic Engagement, Librarian for Chemistry, and Chemical and Biological Engineering, Northwestern University Libraries

ii. Joanne Berger, FDA Library

iii. Daniele Fanelli, Fellow in Quantitative Methodology, Department of Methodology, London School of Economics and Political Science

iv. David Moher, Director, Centre of Journalology, Ottawa Hospital Research Institute

v. Barbara Ruggeri, Life \& Health Sciences Librarian, Carroll University

E. End User Tools \& Services: How do we get retractions and other post-publication updates into the workflow of end users?

a. Google Doc Day 2 - E - End User Tools \& Services

b. Group

i. Michele Avissar-Whiting, Editor in Chief, Research Square

ii. Yuanxi Fu, University of Illinois at Urbana-Champaign

iii. Josh Greenberg, Program Director, Alfred P. Sloan Foundation

iv. Karen Hanus, Director, Advocate Aurora Library, St. Luke's Medical Center

v. Tzu-Kun (Esther) Hsiao, University of Illinois at Urbana-Champaign

vi. Josh Nicolson, Co-founder and CEO scite

vii. Elizabeth Suelzer, User Education and Reference Librarian, Medical College of Wisconsin

viii. Nicole Theis-Mahon, Liaison Librarian \& Health Sciences Collection Coordinator, Health Sciences Library University of Minnesota

F. Standards Development Process: What standards development process will help stakeholders agree on the problems posed and then reach consensus on approaches and potential solutions?

a. Google Doc Day 2 - F - Standards Development Process

b. Group

i. IJsbrand Jan Aalbersberg, Chair of the Standards and Technology Executive Committee of STM; SVP Research Integrity, Elsevier

ii. Elisabeth Bik, Microbiome and Science Integrity consultant 
iii. Dan Kulp, Director, Editorial Development for the journals of the American Chemical Society

iv. Christopher Lehmann, Research Integrity Officer, University of Illinois, Urbana-Champaign

v. Alice Meadows, Director of Community Engagement, NISO

vi. Nathan Woods, University of Illinois at Urbana-Champaign

G. Taxonomy Requirements: In order to implement a taxonomy (of retraction or of post-publication updates) what needs to be true of the taxonomy? What is needed for its successful adoption across scientific publishing?

a. Google Doc Day 2 - G - Taxonomy Requirements

b. Group

i. Geoff Bilder, Director of Technology and Research, Crossref

ii. Nicholas DeVito, Doctoral Researcher, EBM DataLab, University of Oxford

iii. Annette Flanagin, Executive Managing Editor and Vice President, Editorial Operations, JAMA and The JAMA Network, and Executive Editor, JAMAevidence

iv. David Gillikin, Chief, Bibliographic Services Division, National Library of Medicine

v. Eefke Smit, Director of Standards and Technology, International Association of STM Publishers

BREAK - 15 MINUTES

6:50-7:05 AM (PT) | 7:50-8:05 AM (MT) | 8:50-9:05 AM (CT) | 9:50-10:05 AM (ET) | 2:50-3:05

PM (UK) | 3:50-4:05 PM (CET)

\section{Min Specs (Continue in same breakout groups, $30 \mathrm{~min}$ )}

7:05-7:35 AM (PT) | 8:05-8:35 AM (MT) | 9:05-9:35 AM (CT) | 10:05-10:35 AM (ET) | 3:05-3:35 PM (UK) | 4:05-4:35 PM (CET)

In the same groups from Part I, using the same Google Doc:

1. Quickly generate a list of all the must-dos and must-not-do's that should be considered to achieve a successful outcome to the proposed actions in the first session. (5-10 min)

2. Reduce the list to the absolute minimum by dropping anything that is not absolutely essential. Ask "If we violated this spec, could we still achieve our purpose?" If the answer is "yes" - drop it. (20 min) 
BREAK - 15 MINUTES

7:35-7:50 AM (PT) | 8:35-8:50 AM (MT) | 9:35-9:50 AM (CT) | 10:35-10:50 AM (ET) | 3:35-3:50

PM (UK) | 4:35-4:50 PM (CET)

\title{
What? So What? Now What? Debrief (Plenary, $40 \mathrm{~min}$ )
}

7:50-8:30 AM (PT) | 8:50-9:30 AM (MT) | 9:50-10:30 AM (CT) | 10:50-11:30 AM (ET) | 3:50-4:30 PM (UK) | 4:50-5:30 PM (CET)

1. What? What happened in the small group discussions? What did you notice, what stood out?

2. So What? Why is this important? Where is there consensus? What solutions have enough support to be feasible?

3. Now What? What actions make sense? What solutions should we as a group consider? What are the next steps?

\author{
BREAK - 15 MINUTES \\ 8:30-8:45 AM (PT) | 9:30-9:45 AM (MT) | 10:30-10:45 AM (CT) | 11:30-11:45 AM (ET) | 4:30- \\ 4:45 PM (UK) | 5:30-5:45 PM (CET)
}

\section{5\% Solution (Individual brainstorming \& small breakout group discussions, $45 \mathrm{~min}$ )}

8:45-9:30 AM (PT) | 9:45-10:30 AM (MT) | 10:45-11:30 AM (CT) | 11:45 AM-12:30 PM (ET) | 4:45-5:30 PM (UK) | 5:45-6:30 PM (CET)

STRUCTURE: "What is your 15 percent? Where do you have discretion and freedom to act? What can you do without more resources or authority?"

1. 5 minutes alone to generate your own list

2. Individuals share their ideas with the small group one at a time (3 min each)

3. Group members provide a consultation to one another - asking clarifying questions and offering advice (5-7 min per person) 
4. Share in Google Doc: Day 2 15\% Solution Ideas or email MT Campbell $<$ mtc@illinois.edu>

GROUPS:

1. (moved)

2. Ashley Farley, Bill \& Melinda Gates Foundation; Elizabeth Suelzer, Medical College of Wisconsin; Christopher Lehmann, University of Illinois, UrbanaChampaign; Stephanie Boughton, Cochrane; Katie Funk, PubMed Central.

3. Annette Flanagin, JAMA and The JAMA Network; Josh Greenberg (part of this time), Alfred P. Sloan Foundation; Joerg Heber, PLOS and PLOSOne; Eefke Smit, International Association of STM Publishers.

4. Dan Kulp, American Chemical Society; Alice Meadows (part of this time), NISO; Ivan Oransky, Retraction Watch; Sarah Robbie, Taylor and Francis.

5. IJsbrand Jan Aalbersberg, Elsevier; Elisabeth Bik, Microbiome and Science Integrity consultant; Deborah Poff, COPE; Randy Townsend, American Geophysical Union.

6. (moved)

7. David Gillikin, National Library of Medicine; Francesca Grifo, Environmental Protection Agency; Kathrin McConnell, FDA Library; Capt Stephen Gonsalves, US Office of Research Integrity.

8. Caitlin Bakker, Health Sciences Library Univ of Minnesota; Kathryn Kaiser, University of Alabama Birmingham; David Moher, Ottawa Hospital Research Institute; Nicholas DeVito, University of Oxford.

9. (moved)

10. Michele Avissar-Whiting, Research Square; Geoff Bilder, Crossref; James Leung, Clarivate Analytics; Jodi Schneider, University of Illinois at Urbana-Champaign; John Seguin, Third Iron LLC.

11. Daniele Fanelli, London School of Economics and Political Science; Nicole TheisMahon, University of Minnesota; Dmitry Malkov, Sussex University.

12. Patricia Feeney, Crossref; Stacey Lavelle, Aries Systems Corporation; Josh Nicholson, scite.

13. Elsa Alvaro, Northwestern University Libraries; Joanne Berger, FDA Library; Karen Hanus, Advocate Aurora Library; Barbara Ruggeri, Carroll University.

\title{
Wrap up and looking ahead to Day 3 (Plenary, $20 \mathrm{~min}$ )
}

\author{
9:40-10:00AM (PT) | 10:40-11:00 AM (MT) | 11:40 AM-12:00 PM (CT) | 12:40-1:00 PM (ET) | \\ 5:40-6:00 PM (UK) | 6:40-7:00 PM (CET)
}


Monday November 16th

\section{DAY 3 Commitment to Action}

\section{Welcome and Review of Agenda \& Logistics (Plenary, $15 \mathrm{~min}$ )}

6:00-6:15 AM (PT) | 7:00-7:15 AM (MT) | 8:00-8:15 AM (CT) | 9:00-9:15 AM (ET) | 2:00-2:15 PM (UK) | 3:00-3:15 PM (CET)

\section{Planning for collaboration and action (Breakout groups, $45 \mathrm{~min}$ )}

6:15-7:00AM (PT) | 7:15-8:00 AM (MT) | 8:15-9:00 AM (CT) | 9:15-10:00 AM (ET) | 2:15-3:00 PM (UK) | 3:15-4:00 PM (CET)

STRUCTURE: Groups will be determined by survey responses Day 3 Agenda Setting; please answer the survey if you haven't.

Each group will have a Google Doc to guide the discussion as follows:

How do we move the implementation of this recommendation forward?

1. Who needs to collaborate and what is their role? (10 $\mathrm{min})$

2. What resources are needed? (10 min)

3. What collaborative action can we take to make progress? (15 $\mathrm{min}$ )

4. Do we need more information or participation from other participants?

GROUPS: 
A. Support resources and standards to create open, sustainable non-proprietary databases with APIs to track and disseminate retraction statuses.

a. Google Doc Day 3 - A - Database

b. Group

i. Elsa Alvaro, Director of Academic Engagement, Librarian for Chemistry, and Chemical and Biological Engineering, Northwestern University Libraries

ii. Geoff Bilder, Director of Technology and Research, Crossref

iii. Halle Burns, Data Librarian \& Instructor, University of Nevada Las Vegas

iv. Ashley Farley, Program Officer, Knowledge \& Research Services, Bill \& Melinda Gates Foundation

v. James Leung, Product Director, Clarivate

vi. Kathie McConnell, Director, FDA Library, Office of Information Management and Technology

vii. Ivan Oransky, Co-Founder of Retraction Watch \& Editor in Chief, Spectrum, Distinguished Writer In Residence at New York University's Carter Journalism Institute, and president of the Association of Health Care Journalists

viii. John Seguin, President \& Chief Librarian, Third Iron LLC

ix. Sean Takats, Professor/Chief Scientist, University of Luxembourg; Director of Zotero

B. Recommend retraction metadata statuses that can be adopted by all stakeholders.

a. Google Doc Day 3 - B - Retraction Metadata

b. Group

i. Elisabeth Bik, Microbiome and Science Integrity consultant

ii. Helena Cousijn, Community Engagement Director, DataCite

iii. Jennifer Deal, Librarian Lead and Institutional Repository Manager, Advocate Aurora Library

iv. Stephen Gonsalves, Division of Education and Integrity, US Office of Research Integrity

v. Randi Proescholdt, University of Illinois, Urbana-Champaign

C. Recommend a taxonomy of retraction statuses that can be adopted by all stakeholders.

a. Google Doc Day 3 - C - Retraction Taxonomy

b. Group

i. Yoss Arianlou, University of Illinois, Urbana-Champaign

ii. Patricia Feeney, Head of Metadata, Crossref

iii. Annette Flanagin, Executive Managing Editor and Vice President, Editorial Operations, JAMA and The JAMA Network, and Executive Editor, JAMAevidence

iv. Katie Funk, Program Manager, PubMed Central 
v. Karen Hanus, Director, Advocate Aurora Library

vi. Joerg Heber, Editorial Director PLOS and Editor-in-Chief PLOS ONE

vii. Stacey Lavelle, Senior Business Analyst, Aries Systems Corporation

viii. Dmitry Malkov, master's student, Science Policy Research Unit, University of Sussex

ix. Deborah Poff, Editor-in-Chief, Journal of Academic Ethics; Chair, Trustee Board COPE

x. Eefke Smit, Director of Standards and Technology, International Association of STM Publishers

D. Responsible Conduct of Research (RCR) Education.

a. Google Doc Day 3 - D - RCR Education

b. Group

i. Michele Avissar-Whiting, Editor-in-Chief, Research Square

ii. Joanne Berger, Lead Librarian, U.S. Food and Drug Administration

iii. Nicholas De Vito, Doctoral Researcher, EBM DataLab, University of Oxford

iv. Tzu-Kun (Esther) Hsiao, University of Illinois, Urbana-Champaign

v. Kathryn Kaiser, Assistant Professor, Dept of Health Behavior, University of Alabama Birmingham

vi. Barbara Ruggeri, Life and Health Sciences Librarian, Carroll University

E. Strategy \& ownership for standards and best practice development.

a. Google Doc Day 3 - E - Standards Development

b. Group

i. Tom Heyman, Assistant Professor, Social and Behavioural Sciences, Leiden University

ii. Daniel Kulp, Director, Editorial Development for the journals of the American Chemical Society \& COPE Vice-Chair

iii. Christopher Lehmann, Research Integrity Officer, University of Illinois, Urbana-Champaign

iv. Alice Meadows, Director of Community Engagement, NISO

v. Katrina Pickersgill, Executive Peer Review Manager, SAGE Publications, Ltd. (?)

vi. Bruce Rosenblum, Vice President of Content \& Workflow Solutions, Inera Inc

vii. Nathan Woods, University of Illinois, Urbana-Champaign

F. Streamlining the retraction process.

a. Google Doc Day 3 - F - Streamlining the retraction process

b. Group

i. IJsbrand Jan Aalbersberg, Chair of the Standards and Technology Executive Committee of STM; SVP Research Integrity, Elsevier

ii. Yuanxi Fu, University of Illinois, Urbana-Champaign 
iii. Sarah Robbie, Head of Research Integrity and Ethics, Taylor and Francis

iv. Liz Suelzer, User Education and Reference Librarian, Medical College of Wisconsin

G. Public awareness about how retraction is a part of science

a. Google Doc Day 3 - G - Public awareness about retraction

b. Group

i. Monya Baker, Senior Editor, Comment Desk, Nature magazine (plenary)

ii. Daniele Fanelli, Fellow in Quantitative Methodology, Department of Methodology, London School of Economics and Political Science (part day)

iii. Josh Greenberg, Program Director, Alfred P. Sloan Foundation

iv. Francesca Grifo, Scientific Integrity Official, Environmental Protection Agency

v. Josh Nicholson, Co-founder and CEO scite

vi. Jodi Schneider, University of Illinois, Urbana-Champaign

NISO session:

vii. Caitlin Bakker, Medical School Librarian and Research Services Coordinator, Health Sciences Library, University of Minnesota

viii. Hannah Heckner, Product Strategist, Silverchair

ix. Randy Townsend, Director, Journal Operations at American Geophysical Union

BREAK - 15 MINUTES

7:00-7:15 AM (PT) | 8:00-8:15 AM (MT) | 9:00-9:15 AM (CT) | 10:00-10:15 AM (ET) |3:00-3:15

PM (UK) | 4:00-4:15 PM (CET)

\section{A "Call to Action" Discussion \& Recruiting Session (Plenary, 20 min)}

7:15-7:35 AM (PT) | 8:15-8:35 AM (MT) | 9:15-9:35 AM (CT) | 10:15-10:35 AM (ET) | 3:15-3:35 PM (UK) | 4:15-4:45 PM (CET)

What collaborative action are you taking? Who do you need to recruit from the room?

\section{Do-a-thon (Breakout groups, $30 \mathrm{~min}$ )}


7:45-8:15AM (PT) | 8:45-9:15 AM (MT) | 9:45-10:15 AM (CT) | 10:45-11:15 AM (ET) | 3:45-4:15 PM (UK) | 4:45-5:15 PM (CET)

Continue breakout sessions, now task-focused, with possibly re-configured groups using the same Google Doc:

1. Task: Now that you have set a task, work in the same or reconfigured groups. Further develop your collaborative action.

Even if you can't finish now, you can go home with a working draft.

GROUPS (same topics as above + any new topics that emerge):

A. A stand-alone, non-proprietary database to track and disseminate retraction status.

a. Google Doc (same as above)

b. Group

B. All stakeholders should adopt standard retraction metadata.

a. Google Doc (same as above)

b. Group

C. Develop a taxonomy of retraction statuses shared and adopted by all stakeholders.

a. Google Doc (same as above)

b. Group

D. Responsible Conduct of Research (RCR) Education

a. Google Doc (same as above)

b. Group

E. Strategy \& Ownership for standards and best practice development

a. Google Doc (same as above)

b. Group

F. Streamlining the retraction process.

a. Google Doc (same as above)

b. Group

G. Public awareness about how retraction is a part of science

\section{BREAK - 15 MINUTES}

8:15-8:30 AM (PT) | 9:15-9:30 AM (MT) | 10:15-10:30 AM (CT) | 11:15-11:30 AM (ET) 4:15-4:30 PM (UK) | 5:15-5:30 PM (CET)

\section{Dissemination Plans and White Paper Development (Plenary, 20 min)}


8:30-8:50 AM (PT) | 9:30-9:50 AM (MT) | 10:30-10:50AM (CT) | 11:30-11:50 AM (ET) | 4:30-

4:50M (UK) | 5:30-5:50 PM (CET)

\section{Working Session (30 min)}

9:00-9:30 AM (PT) | 10:00-10:30 AM (MT) | 11:00-11:30 AM (CT) | 12:00-12:30 PM (ET) | 5:00-

5:30 PM (UK) |6:00-6:30 PM (CET)

Continue the do-a-thon. Or start a new collaborative task.

\section{Final Wrap Up Plenary (15 min)}

9:30-9:45M (PT) | 10:30-10:45 AM (MT) | 11:30--11:45 AM (CT) | 12:30-12:45 PM (ET) | 5:30-

5:45 PM (UK) | 6:30-6:45 PM (CET) 


\section{Workshop Participant List}

- IJsbrand Jan Aalbersberg, PhD, Chair of the Standards and Technology Executive Committee of STM; SVP Research Integrity, Elsevier

- Elsa Alvaro, PhD, Director of Academic Engagement, Librarian for Chemistry, and Chemical and Biological Engineering, Northwestern University Libraries

- Michele Avissar-Whiting, PhD, Editor-in-Chief, Research Square

- Monya Baker, Ed.M., Senior Editor, Comment Desk, Nature magazine

- Caitlin Bakker, MSLIS, Medical School Librarian and Research Services Coordinator, Health Sciences Library, University of Minnesota

- Joanne Berger, Lead Librarian, U.S. Food and Drug Administration

- Lisa Bero, PhD, Chief Scientist, Center for Bioethics and Humanities; Professor of Medicine and Public Health, University of Colorado Anschutz Medical Campus

- Elisabeth Bik, PhD, Microbiome and Science Integrity consultant 
- Geoff Bilder, Director of Technology and Research, Crossref

- Stephanie Boughton, Research Integrity Editor, Cochrane

- Helena Cousijn, PhD, Community Engagement Director, DataCite

- Jennifer Deal, MA, MLIS, Librarian Lead and Institutional Repository Manager, Advocate Aurora Library

- Nicholas De Vito, Doctoral Researcher, EBM DataLab, University of Oxford

- Daniele Fanelli, PhD, Fellow in Quantitative Methodology, Department of Methodology, London School of Economics and Political Science

- Ashley Farley, MSLIS, Program Officer, Knowledge \& Research Services, Bill \& Melinda Gates Foundation

- Patricia Feeney, MLIS, Head of Metadata, Crossref

- Annette Flanagin, RN, MA, FAAN, Executive Managing Editor and Vice President, Editorial Operations, JAMA and The JAMA Network, and Executive Editor, $\underline{\text { JAMAevidence }}$

- Kathryn Funk, MSLIS, Program Manager, PubMed Central

- CAPT Stephen Gonsalves, PhD, Division of Education and Integrity, US Office of Research Integrity

- Josh Greenberg, PhD, Program Director, Alfred P. Sloan Foundation

- Francesca Grifo, PhD, Scientific Integrity Official, Environmental Protection Agency

- C.K. Gunsalus, J.D., Director, National Center for Professional \& Research Ethics, Professor Emerita of Business, and Research Professor at the Coordinated Sciences Laboratory, University of Illinois at Urbana-Champaign

- Karen Hanus, MLIS, AHIP, Director, Advocate Aurora Library 
- Joerg Heber, PhD, Editorial Director PLOS and Editor-in-Chief PLOS ONE

- Hannah Heckner, Product Strategist, Silverchair

- Tom Heyman, PhD, Assistant Professor, Social and Behavioural Sciences, Leiden University

- Kathryn Kaiser, PhD, Assistant Professor, Dept of Health Behavior, University of Alabama Birmingham

- Daniel T. Kulp, PhD, Director, Editorial Development for the journals of the American Chemical Society \& COPE Vice-Chair

- Stacey Lavelle, Senior Business Analyst, Aries Systems Corporation

- Christopher Lehmann, PhD, Research Integrity Officer, University of Illinois, UrbanaChampaign

- James Leung, PhD, Product Director, Clarivate

- Dmitry Malkov, master's student, Science Policy Research Unit, University of Sussex

- Aaron Manka, PhD, Director, Research Integrity and Administrative Investigations, National Science Foundation - Office of Inspector General

- Michael Markie, Publishing Director, Life Sciences, F1000

- Kathrin McConnell, Director, FDA Library, Office of Information Management and Technology

- Alice Meadows, Director of Community Engagement, $\underline{\text { NISO }}$

- David Moher, PhD, Director, Centre of Journalology, Ottawa Hospital Research Institute; Associate Professor, School of Epidemiology and Public Health, University of Ottawa

- Josh Nicholson, PhD, Co-founder and CEO scite

- Ivan Oransky, MD, Co-Founder of Retraction Watch \& Editor in Chief of Spectrum, Distinguished Writer In Residence at New York University's Carter Journalism Institute, 
and president of the Association of Health Care Journalists

- Laura Paglione, Project Lead, Metadata2020

- Katrina Pickersgill, Executive Peer Review Manager, SAGE Publications, Ltd.

- Deborah Poff, PhD, Editor-in-Chief, Journal of Academic Ethics; Chair, Trustee Board COPE

- Jessica Polka, PhD, Executive Director, ASAPbio

- Sarah Robbie, Head of Research Integrity and Ethics, Taylor and Francis

- Pamela Ronald, PhD, Distinquished Professor, Department of Plant Pathology, University of California, Davis

- Bruce D. Rosenblum, Vice President of Content \& Workflow Solutions, Inera Inc

- Barbara Ruggeri, MLIS, AHIP, Life and Health Sciences Librarian, Carroll University

- John Seguin, President \& Chief Librarian, Third Iron LLC

- Eefke Smit, MA, Director of Standards and Technology, International Association of STM Publishers

- Elizabeth Suelzer, MSLIS, User Education and Reference Librarian, Medical College of Wisconsin

- Sean Takats, PhD, Professor/Chief Scientist, University of Luxembourg; Director of Zotero

- Nicole Theis-Mahon, MSLIS, AHIP, Liaison Librarian \& Health Sciences Collection Coordinator, Health Sciences Library, University of Minnesota

- Randy Townsend, MA, Director, Journal Operations at American Geophysical Union 


\section{CRediT Contributor Statement for Appendix D: Stakeholder Consultation Process}

Yoss Arianlou: Investigation; Halle Burns: Investigation; Mary Terese Campbell: Project administration, Writing - original draft, Writing - review \& editing; Yuanxi Fu: Investigation; TzuKun (Esther) Hsiao: Investigation; Randi Proescholdt: Investigation; Jodi Schneider: Conceptualization, Data curation, Funding acquisition, Investigation, Methodology, Project administration, Supervision, Writing - original draft, Writing - review \& editing; Nathan D. Woods: Conceptualization, Data curation, Formal analysis, Investigation, Methodology, Supervision, Writing-original draft, Writing - review \& editing.

\section{BIBLIOGRAPHY}

Lipmanowicz, H., \& McCandless, K. (2014). The Surprising Power of Liberating Structures:

Simple Rules to Unleash a Culture of Innovation. Liberating Structures Press. 


\section{Appendix E: Existing Citation Standards for Retracted Publications}

Randi Proescholdt, Jodi Schneider, and the RISRS Team

To accompany "Recommendations from the Reducing the Inadvertent Spread of Retracted Science: Shaping a Research and Implementation Agenda Project"

July 2021

\section{AMA/American Medical Association Manual of Style}

Guidelines:

Instructions are not fully stated, but examples are provided: "If the reference citation is to an article that has since been retracted or retracted and replaced, to an article for which there is an expression of concern, or to the retraction notice itself, use the appropriate example below...Note: DOls do not follow the citation of the retracted article" (AMA Manual of Style Committee, 2020).

\section{Example:}

Reuben SS. Postoperative Modulation of Central Nervous System Prostaglandin E2 by Cyclooxygenase Inhibitors after Vascular Surgery. Anesthesiology. 2006;104(3):6.

Retracted in: Anesthesiology. 2009;110(3):689-689. doi:10.1097/ALN.0b013e31819e9cdd

\section{APA/Publication Manual of the American Psychological Association}

Guidelines:

"To create a reference for a retracted article, you can repurpose the parentheses found after a reference that includes original publication information, as can be seen in Examples 21 and 26 on pages 203-204 of the Publication Manual of the American Psychological Association, sixth edition. Instead, provide the information on the article's retraction notice in the parentheses" (2013)

\section{Example:}

Reuben, S. S. (2006). Postoperative Modulation of Central Nervous System Prostaglandin E2 by Cyclooxygenase Inhibitors after Vascular Surgery. Anesthesiology, 104(3), 6.

(Retraction published March 1, 2009, Anesthesiology, 110, p. 689) 


\section{NLM/Citing Medicine}

Guidelines:

"To indicate that an article had a subsequent retraction notice published, begin by citing the article. Enter the phrase "Retraction in: " followed by the names of the authors, the journal title abbreviation, date of publication, volume, issue, and location (pagination). Use the same punctuation and format as you would for the article itself" (Patrias \& Wendling, 2018).

Example:

Reuben SS. Postoperative Modulation of Central Nervous System Prostaglandin E2 by Cyclooxygenase Inhibitors after Vascular Surgery. Anesthesiology. 2006;104(3):6. Retraction in: Anesthesiology. 2009 Mar 1;110(3):689-689.

\section{CRediT contributor statement for Appendix E: Existing Citation Standards for Retracted Publications}

Randi Proescholdt: Conceptualization, Investigation, Methodology, Writing - original draft, Writing - review \& editing; Jodi Schneider: Conceptualization, Funding acquisition, Supervision; Writing - review \& editing.

\section{BIBLIOGRAPHY}

AMA Manual of Style Committee. (2020, February). Retractions and Expressions of Concern. AMA Manual of Style. 10.1093/jama/9780190246556.001.0001

Patrias, K., \& Wendling, D. (2018). Citing Medicine (2nd ed.). National Library of Medicine (US).

Stefanie. (2013, January 18). Retraction Action. APA Style 6th Edition Blog. https://blog.apastyle.org/apastyle/2013/01/retraction-action.html 